SENSITIVITY ANALYSIS AND OPTIMAL DESIGN OF CONVENTIONAL AND MAGNNETORHEOLOGICAL FLUID BRAKES

A Dissertation
presented to
the Faculty of the Graduate School
at the University of Missouri-Columbia

In Partial Fulfillment

of the Requirements for the Degree

Doctor of Philosophy

by

SALWAN OBAID WAHEED KHAFAJI

Dr. Noah Manring, Dissertation Supervisor

May 2018 
The undersigned, appointed by the dean of the Graduate School, have examined the dissertation entitled

\section{SENSITIVITY ANALYSIS AND OPTIMAL DESIGN OF CONVENTIONAL AND MAGNETORHEOLOGICAL FLUID BRAKES}

presented by Salwan Obaid Waheed Khafaji

a candidate for the degree of doctor of philosophy and hereby certify that it is worthy of acceptance.

Professor Manring Noah

Professor Craig Kluever

Professor Ahmed Sherif Al-Gizawy

Professor Banks William

Professor Steven Borgelt 


\section{ACKNOWLEDGEMENTS}

I am greatly thankful to my advisor Dr. Noah Manring for spending his time advising and helping me finish this thesis. I would like to express my deepest appreciation to my advisor; without his patient guidance, I wouldn't have grown as a good researcher and gotten to this step. His continuous encouragement and valuable suggestions and recommendation on my work helped a lot. He is a great professor who cares about his students' growth. Because of his effort and continuous encouragement, I have gotten better not only at research, but also at formal writing and academic presentation. I thank him for being such a great advisor.

Second, I would like to thank my sponsor, The Higher Committee for Educational Development in IRAQ (HCED IRAQ) for their financial support thorough out my study. I also want to thank my Department back home (Mechanical Engineering Department at the University of Babylon/ IRAQ) for giving me the chance to develop my academic experience. In addition, I would like to thank Department of Mechanical and Aerospace Engineering at University of Missouri for considering me as a graduate student.

I would like to thank my wonderful family, brothers Wegdan, Mahran, Waheed, Samir and my sisters and friends from all over the world for their help and support. I want to express my deep gratitude toward my mother Mrs. Fadilah Khalil and my father Mr. Obaid Waheed who I kept in my heart for their unconditional love and support before and during my graduate study. They keep giving me the hope to study and the love to do that. I never forgot them and kept remembering them every day. I do not want to forget Fatima, Hasan, Hussein, and the little happy baby John who helped me a lot. My parents 
and family have been taking care of me throughout my life and for advising and providing me support although we live overseas. I also want to thank other relatives as well as friends. Many thanks to Kathy Johnson Gilmore, Kathy Earhart Smith, and Sarah Wellsand for their help and taking care of my wife and kids.

Finally, I am greatly thankful to ALLAH, the lord of whole creation for selecting me to get this opportunity to come to US and getting my degree and know this great community. 


\section{TABLE OF CONTENTS}

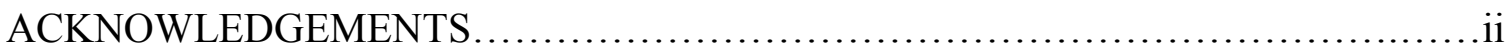

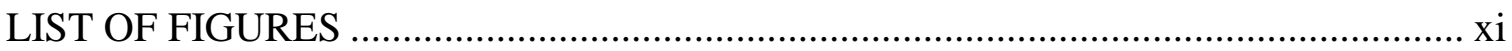

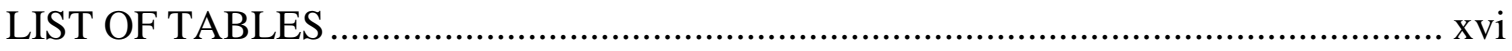

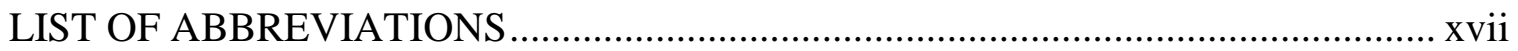

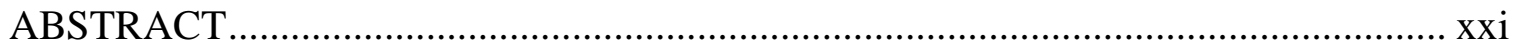

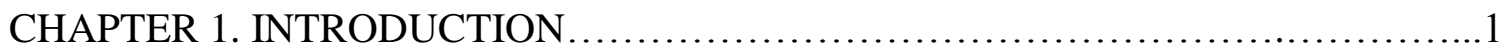

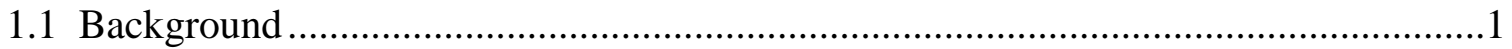

1.1.1 Conventional and Electrical Brakes ...........................................................

1.1.2 Magnetorheological Fluid Brake....................................................................

1.2 Magnetorheological Fluid History and Background..........................................

1.3 Physical Composition of a Magnetorheological Fluid...........................................

1.4 Magnetorheological Fluid in Applications .........................................................

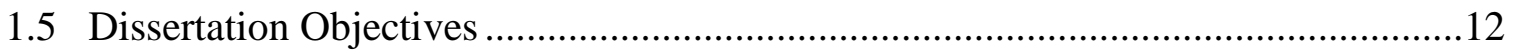

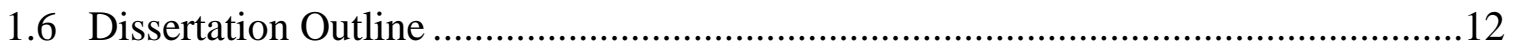

CHAPTER 2. LITERATURE REVIEW ........................................ 15

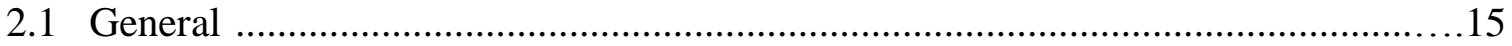

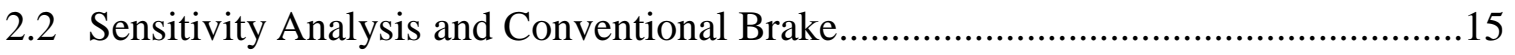

2.3 Analysis and Optimal Design of Magnetorheological Fluid Brake.........................20

2.4 Magneto-Rheological Fluid Brake and Fluid Behavior.......................................31

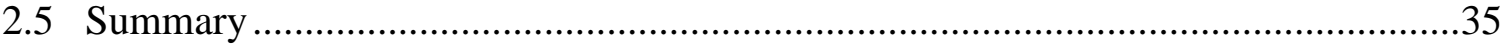




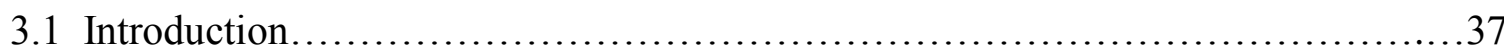

3.2 Sensitivity Analysis of a Single Shoe Conventional Drum Brake............................37

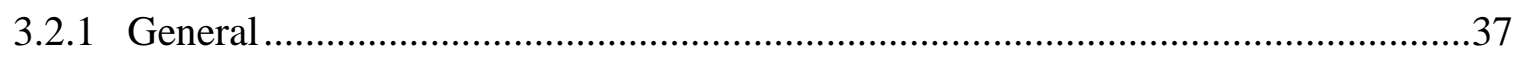

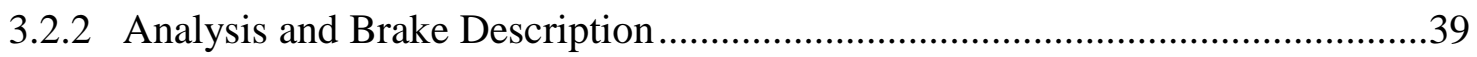

3.2.3 Free-Body-Diagram Analysis ...............................................................40

3.2.4 Nondimensional Form of Conventional Brake ............................................43

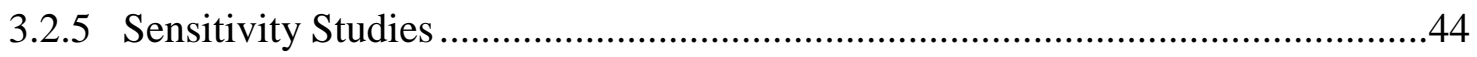

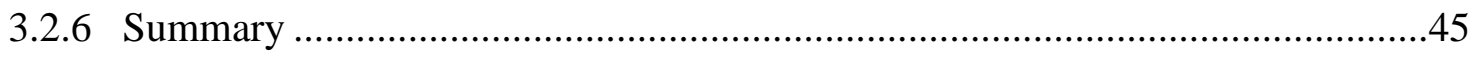

3.3 Sensitivity Analysis of Magnetorheological Fluid Brake (MRF) ...........................45

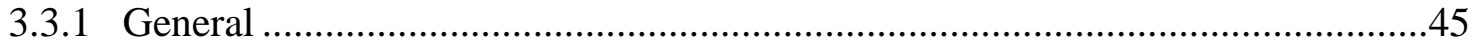

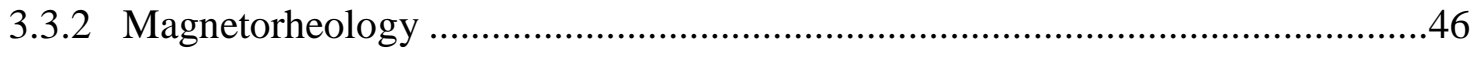

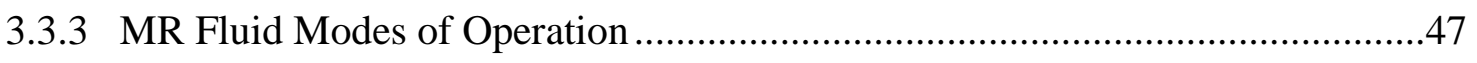

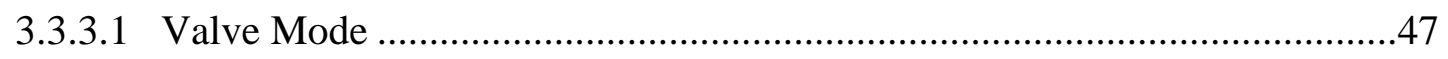

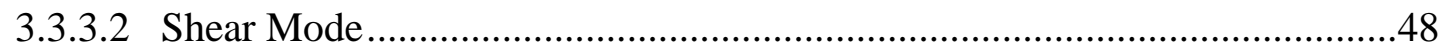

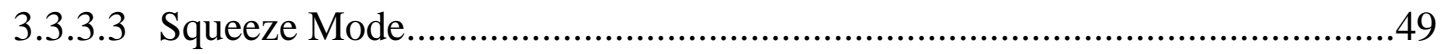

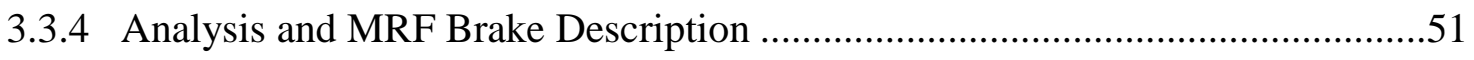

3.3.5 Principle of MRF Brake and Free Body Diagram Analysis ...........................52

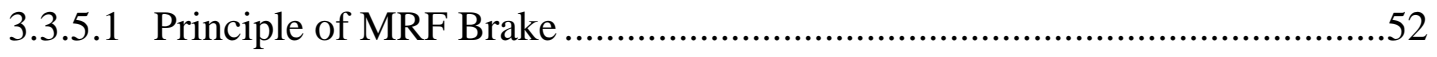

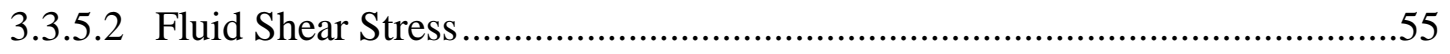


3.3.5.3 Fluid Shear Strain

3.3.5.4 Shear Strain Rate of MRF Brake .58

3.3.6 Magnetic Circuit Design .60

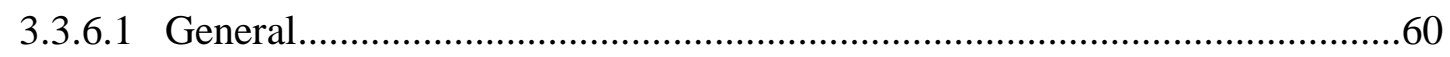

3.3.6.2 Candidate Material used for MRF Brake ...............................................64

3.3.6.3 Magnetic Field Intensity and Brake Dimensions ...................................64

3.3.6.3.1. Magnetic Reluctance...........................................65

3.3.6.3.2. Magnetic Reluctance of MRF Brake...............................67

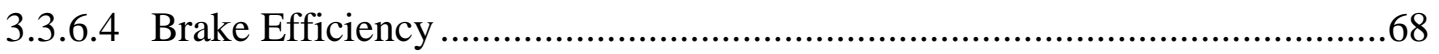

3.3.7 Sensitivity Analysis for magnetorheological fluid (MRF) Brake and Magnetic

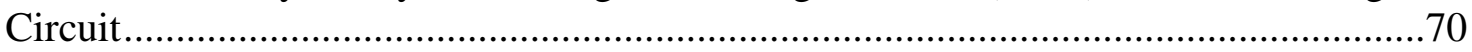

3.3.7.1 Nondimensional Form of MRF braking torque ...................................... 70

3.3.7.2 Sensitivity Studies of MRF braking torque ............................................. 71

3.3.7.3 Nondimensional Form of MRF Brake Magnetic Circuit............................72

3.3.7.4 Sensitivity Studies of MRF Magnetic Circuit .........................................74

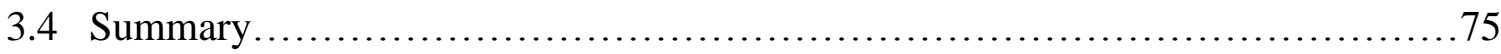

CHAPTER 4. OPTIMAL DESIGN USING TAGUCHI METHOD ....................77

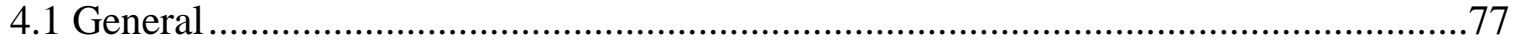

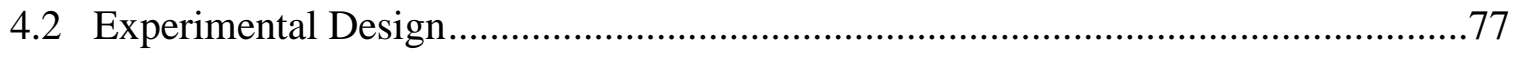

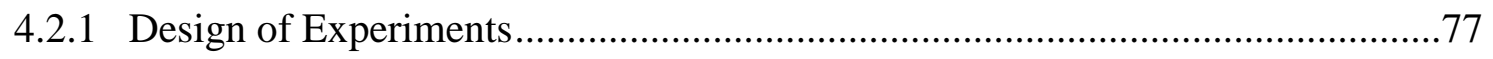

4.2.2 Fundamentals of Design of experiments.................................................... 79 
4.3 Taguchi Design and Orthogonal Array

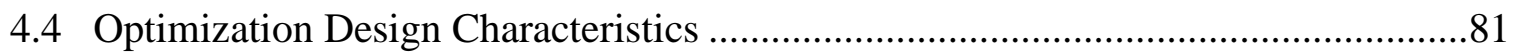

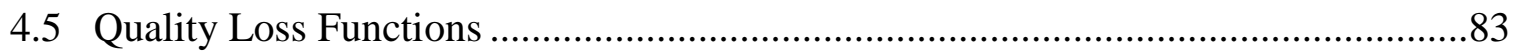

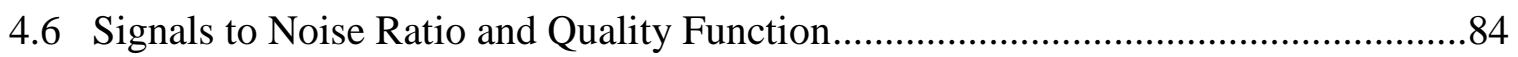

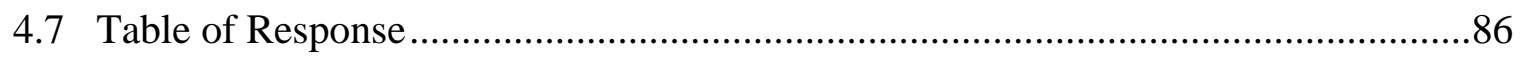

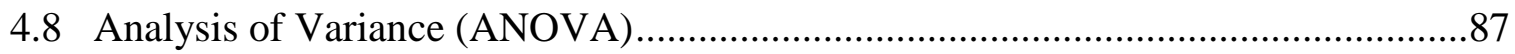

4.9 Taguchi Application for Conventional and Magnetorheological Fluid Brakes..........88

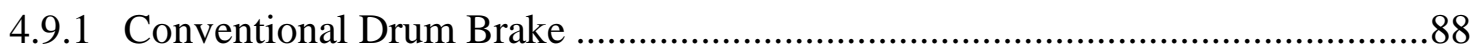

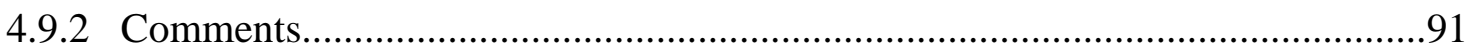

4.9.3 Magnetorheological Fluid Brake..................................................................92

4.9.3.1 Off- Field Braking Torque ...................................................................92

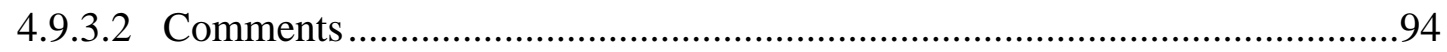

4.9.3.3 On- Field Braking Torque ....................................................................95

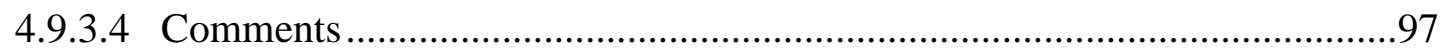

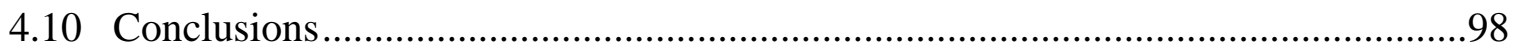

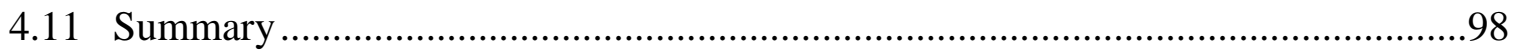

CHAPTER 5. MAGNETORHEOLOGICAL FLUID BRAKE OF VARIABLE FLLUID

THICKNESS (CVFT, NEW OPTIMAL DESIGNS) .................................100

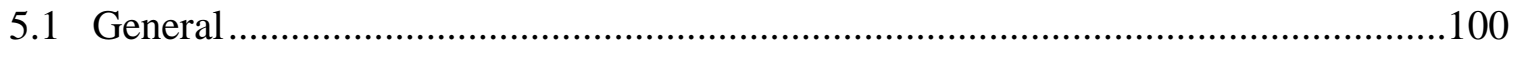

5.2 Magnetic Saturation and Hysteresis loop …………...........................................101

5.3 Continuous Variable Fluid Thickness (Torque) MRF Brake-New Suggested Designs

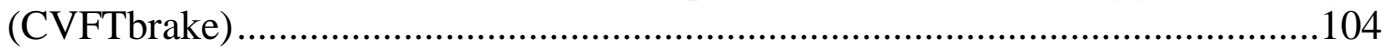


5.3.2 Continuous Variable Fluid Thickness (Torque) of One Variable Fluid Thickness (CVFT1h) 106

5.3.3 Updated Mathematical Model for the CVFT1h. .109

5.3.4 Continuous Variable Fluid Thickness (Torque) of Two Variable Fluid Thicknesses (CVFT2h)... 110

5.3.5 Updated Mathematical Model for the CVFT2h. 113

5.3.6. Simple Calculations 114

5.4 Summary 115

CHAPTER 6. CONTROLLING A MAGNETORHEOLOGICAL (MR) FLUID

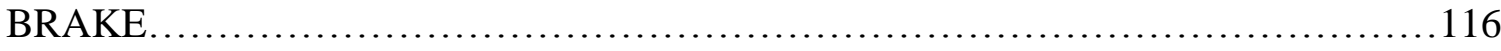

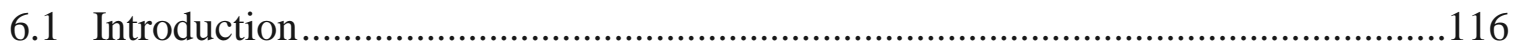

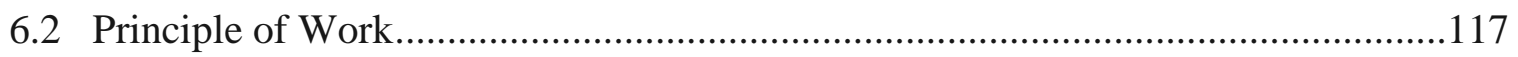

6.2.1 Principle of Work of a Solenoid and the Braking Torque ..............................117

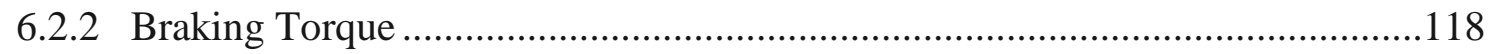

6.3 Mathematical Modeling of an electromechanical Solenoid................................119

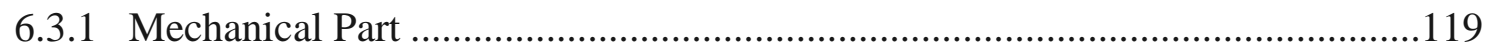

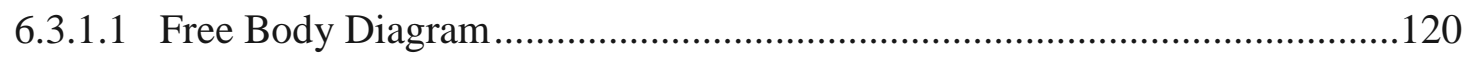

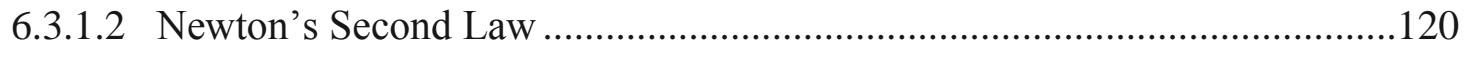

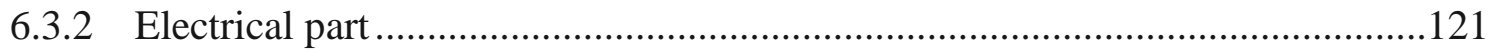

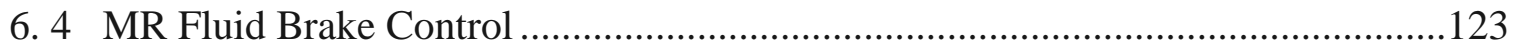

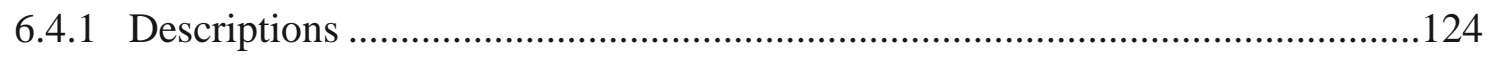


6.4.2.1 Dynamic System ....................................................................................126

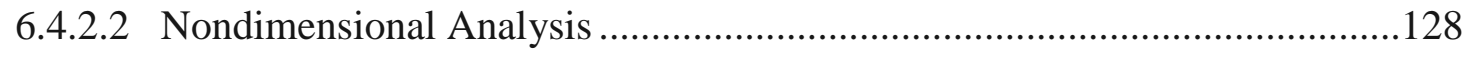

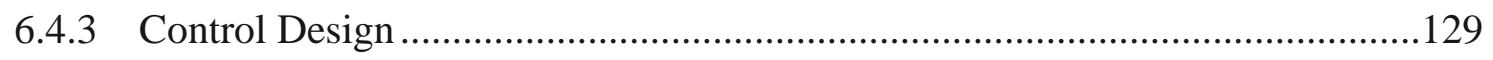

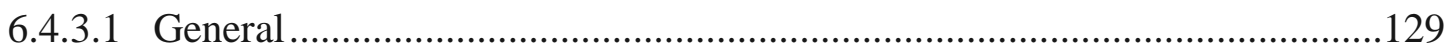

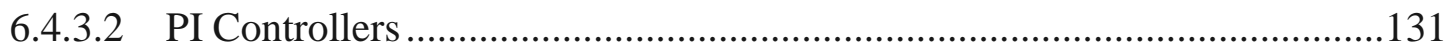

6.4.3.3 Non-Unique Solutions …………………...................................................132

6.4.3.4 Linearization and Control-Gain Selection ……………………..................133

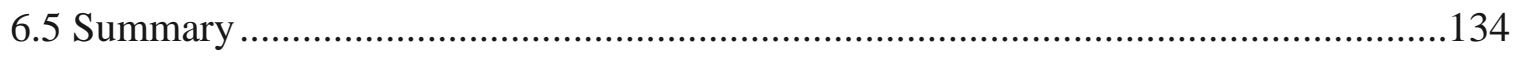

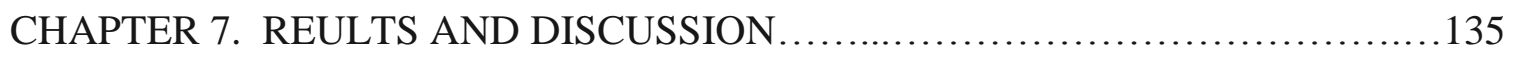

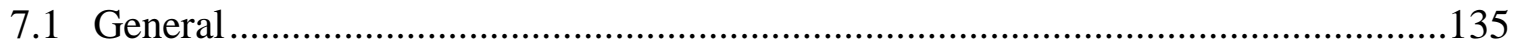

7.2 Sensitivity Analysis of Conventional Drum Brake...............................................138

7.3.1 Viscous and Magnetic Field Braking Torque ..............................................138

7.3.2 Electromagnetic Circuit Characteristics of MRF brake …….............................144

7.3.3 Combined Effect of Brake Radii $\hat{r}_{1}$ and $\hat{r}_{2}$ on Mechanical and Electromagnetic

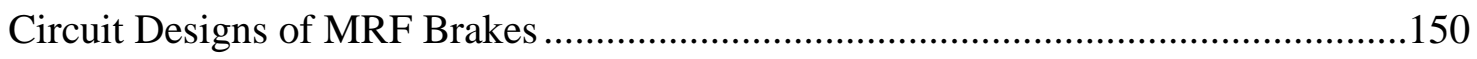

7.4 Controlling a Magnetorheological (MR) fluid brake..............................................150

7.4.1 High Speed Control..............................................................................150

7.4.2 Moderate Speed Control...........................................................................152

7.4.3 Low Speed Control ..................................................... 154

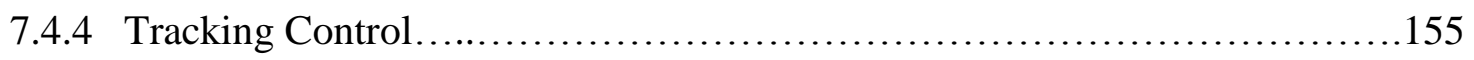


7.5 Summary.

CHAPTER 8. CONCLUSIONS AND FUTURE WORK.............................158

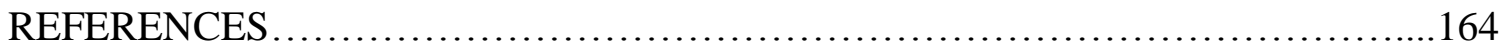

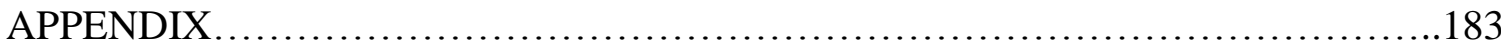

A.1 The Torque Capacity of a MR Fluid Brake Compared to A Frictional Disk Brake.183

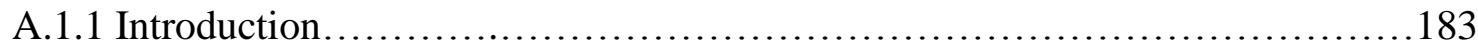

A.1.2 Descriptions of Frictional Disc and MR fluid Brake...........................185

A.1.2.1 Frictional Disk Brake..................................................185

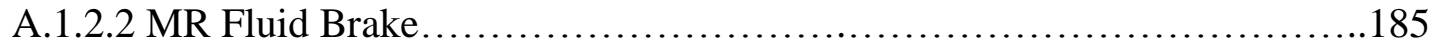

A.1.3 Mathematical Modelling and Analysis.....................................187

A.1.3.1 Frictional Brake Equations........................................187

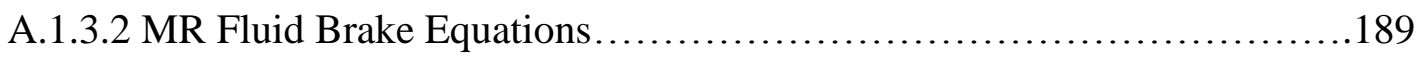

A.1.4 Brake Comparisons..................................................... 190

A.2 Numerical Values for Some Design Parameters...................................192

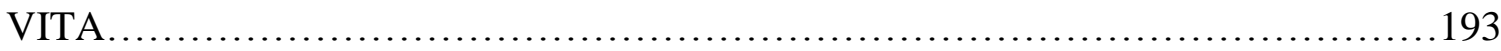




\section{LIST OF FIGURES}

Figure 1-1. Oldest type of brake................................................

Figure 1-1. Wrapped cable brake...............................................

Figure 1-3. Conventional drum and disc brake.....................................

Figure 1-2. A schematic diagram for conventional brake.............................. 4

Figure 1-5. A typical magnetorheological fluid brake................................5

Figure 1-6. A schematic diagram of a typical magnetorheological fluid brake.............6

Figure 1-7. Chain like-structure of a magnetorheological fluid due to an applied magnetic

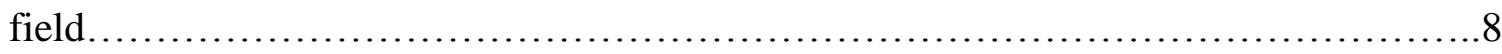

Figure 1-8. Magnetorheological fluid application for seismic and prosthetic devices....11

Figure 3-1. A schematic of the single-shoe drum brake..............................40

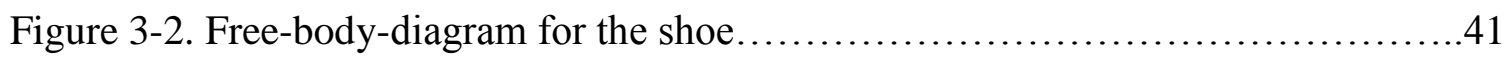

Figure 3-3. Free-body-diagram for the drum..................................43

Figure 3-4. Schematic of a magnetorheological fluid solidifying and blocking a pipe in

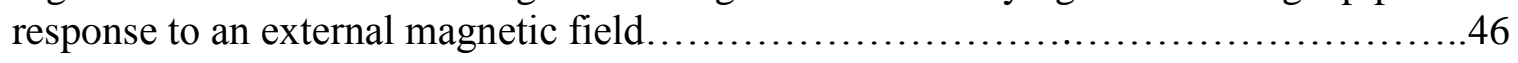

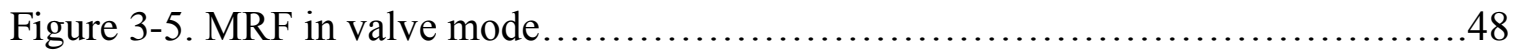

Figure 3-6. MRF in shear mode............................................... 49

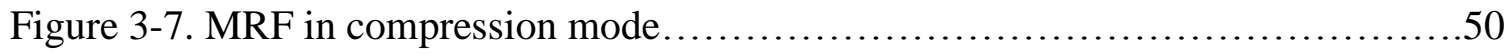

Figure 3-8. A schematic diagram of simple MRF brake ............................51

Figure 3-9. A schematic diagram of a typical magnetorheological fluid brake..........50

Figure 3-10. A schematic showing the geometry of MR fluid brakes and rotary disc

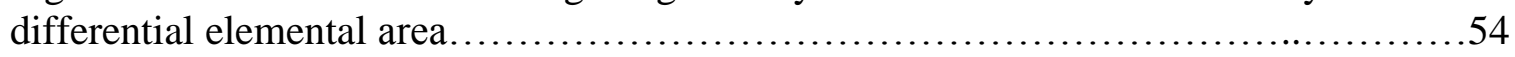

Figure 3-11. Bingham plastic model of an MRF shear stress verses shear rate...........55 
Figure 3-12. Different behaviors of fluid response to shear rate....................57

Figure 3-13. Differential fluid element subjected to shear forces...................57

Figure 3.14. Wounded coil with input current and induced magnetic field lines.........61

Figure 3-15. Magnetic circuit of sections of MRF brake..........................63

Figure $3-16$. Basic quantities of the magnetic circuit............................65

Figure 3-17. Reluctance element of non-uniform flux path.......................67

Figure 4-1. Engineering problem solving scheme.............................. 78

Figure $4-2$. Some of standard orthogonal arrays................................ 81

Figure 4-3. Traditional approach and Taguchi loss function........................83

Figure 4-4. Taguchi loss function, smaller the better and larger the better..............84

Figure 4-5. L4 orthogonal array and their table of response $\ldots \ldots \ldots \ldots \ldots \ldots \ldots \ldots \ldots \ldots . \ldots 6$

Figure 4-6. Signal to noise ratio and best levels for the factors.....................91

Figure 4-7. Signal to noise ratio and best levels for the factors for $\hat{T}_{p} \ldots \ldots \ldots \ldots \ldots . . .64$

Figure 4-8. Signal to noise ratio and best levels for the factors for $\hat{T}_{H} \ldots \ldots \ldots \ldots \ldots \ldots 9$

Figure 5-1. Saturated yield stress of a magnetorheological fluid brake...............101

Figure 5-2. A hysteresis loop for a typical magnetic material, adapted from............ 102

Figure 5-3. A schematic diagram of the suggested design of the MRF brake

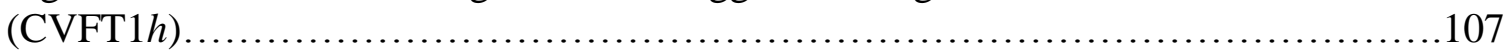

Figure 5-4. Rotaintg and rotaing-translating disks design........................ 108

Figure 5-5. Steps of changing fluid thickness by the actuator movement..............109

Figure 5-6. A schematic diagram of the suggested design of the dynamic MRF brake

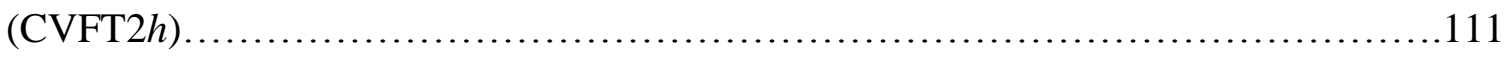

Figure 5-7. Components of the two variable fluid thicknesses brake (CVFT2h).......112

Figure 5-8. Effect of MRF thickness variations on the braking torque................114 
Figure 6-1. A typical electromechanical actuator.............................. 118

Figure 6-2. Schematic Representation for the Mechanical part of the actuator..............120

Figure 6-3. Free body diagram of the equivalent spring-mass system of the solenoid...120

Figure 6-4. A schematic diagram of the electric part of the actuator.................. 122

Figure 6-5. Schematic of the single disk, MR fluid brake..........................125

Figure 6-6. Schematic of the rotating system being controlled by the MR fluid brake...126

Figure 6-7. Block diagram for the MR fluid brake control showing two PI controllers with saturation and a nonlinear relationship for $\hat{h}$

Figure 7-1. Variation in sensitivity coefficients for changes in $\hat{b}$. These results have been

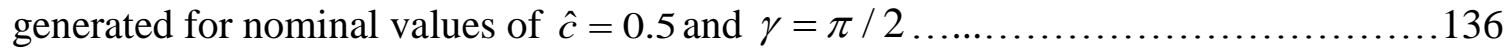

Figure 7-2. Variation in sensitivity coefficients for changes in $\hat{c}$. These results have been

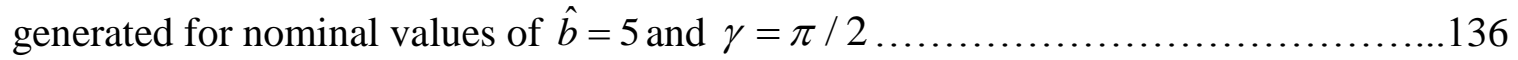

Figure 7-3. Variation in sensitivity coefficients for changes in $\gamma$ (reported in degrees).

These results have been generated for nominal values of $\hat{b}=5$ and $\hat{c}=0.5 \ldots \ldots \ldots \ldots \ldots . .137$

Figure 7-4. Variation in sensitivity coefficients for changes in nondimensional radius $\hat{r}_{i}$. For nondimensional $\left(\hat{T}_{P}\right)$. These results have been generated for positive nominal values only.

Figure 7-5. Variation in sensitivity coefficients for changes in nondimensional radius $\hat{r}_{i}$ for the nondimensional torque $\left(\hat{T}_{H}\right)$. These results have been generated for nominal values of $\hat{k}=0.5, \hat{\beta}=0.5$, and $\hat{H}=1.5$

Figure 7-6. Variation in sensitivity coefficients for changes in nondimensional $\hat{k}_{o}$ for the nondimensional torque $\left(\hat{T}_{H}\right)$. These results have been generated for positive nominal values $\hat{r}_{i}=0.6, \hat{\beta}=0.5$, and $\hat{H}=1.5$

Figure 7-7. Variation in sensitivity coefficients for changes in nondimensional $\hat{\beta}_{o}$ for the nondimensional torque $\left(\hat{T}_{H}\right)$. These results have been generated for positive nominal values $\hat{r}_{i}=0.6, \hat{k}=0.5$, and $\hat{H}=1.5$. 
Figure 7-8. Variation in sensitivity coefficients for changes in nondimensional $\hat{H}_{0}$ for the nondimensional torque $\left(\hat{T}_{H}\right)$. These results have been generated for positive nominal values $\hat{r}_{i}=0.6, \hat{k}=0.5$, and $\hat{\beta}=0.5$

Figure 7-9. Variation in sensitivity coefficients for changes in nondimensional $\hat{H}_{0}$ for the nondimensional torque $\left(\hat{T}_{H}\right)$. These results have been generated for positive nominal values $\hat{r}_{i}=0.6$ and $\hat{k}=0.5$.

Figure 7-10. Variation in sensitivity coefficients for changes in nondimensional radius $\hat{r}_{3}$ for the nondimensional reluctance $\left(\hat{\mathfrak{R}}_{A}\right)$. These results have been generated for nominal values of $\hat{r}_{1}=0.6, \hat{r}_{2}=1$, and $\hat{r}_{4}=1.7$

Figure 7-11. Variation in sensitivity coefficients for changes in nondimensional radius $\hat{r}_{4}$ for the nondimensional reluctance $\left(\hat{\mathfrak{R}}_{A}\right)$. These results have been generated for nominal values of $\hat{r}_{1}=0.6, \hat{r}_{2}=1$, and $\hat{r}_{3}=1.5$.

Figure 7-12. Variation in sensitivity coefficients for changes in nondimensional radius $\hat{r}_{3}$ for the nondimensional reluctance $\left(\hat{\mathfrak{R}}_{B}\right)$. These results have been generated for nominal values of $\hat{r}_{1}=0.6, \hat{r}_{2}=1$, and $\hat{r}_{4}=1.7$

Figure 7-13. Variation in sensitivity coefficients for changes in nondimensional radius $\hat{r}_{3}$ for the nondimensional reluctance $\left(\hat{\mathfrak{R}}_{B}\right)$. These results have been generated for nominal

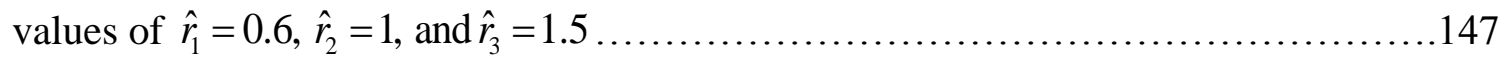

Figure 7-14. Variation in sensitivity coefficients for changes in nondimensional radius $\hat{r}_{1}$ for the nondimensional reluctance $\left(\hat{\mathfrak{R}}_{C}\right.$ and $\left.\hat{\mathfrak{R}}_{D}\right)$. These results have been generated for nominal values of $\hat{r}_{2}=1, \hat{r}_{3}=1.5$, and $\hat{r}_{4}=1.7$

Figure 7-15. Variation in sensitivity coefficients for changes in nondimensional radius $\hat{r}_{2}$ for the nondimensional reluctance $\left(\hat{\mathfrak{R}}_{C}\right.$ and $\left.\hat{\mathfrak{R}}_{D}\right)$. These results have been generated for nominal values of $\hat{r}_{1}=0.6, \hat{r}_{3}=1.5$, and $\hat{r}_{4}=1.7$

Figure 7-16. Transient response illustrating high speed control for $\hat{T}_{L}=1$ and $\hat{\omega}_{d}=2$. The blue line is shaft speed, the red line is MR fluid thickness, and the green line is current 
Figure 7-17. Transient response illustrating moderate speed control with a low-speed initial condition, and where $\hat{T}_{L}=1$ and $\hat{\omega}_{d}=1$. The blue line is shaft speed, the red line is MR fluid thickness, and the green line is current.............................. 152

Figure 7-18. Transient response illustrating moderate speed control with a high-speed initial condition, and where $\hat{T}_{L}=1$ and $\hat{\omega}_{d}=1$. The blue line is shaft speed, the red line is MR fluid thickness, and the green line is current.............................. 153

Figure 7-19. Transient response illustrating low speed control for $\hat{T}_{L}=1$ and $\hat{\omega}_{d}=0$. The blue line is shaft speed, the red line is MR fluid thickness, and the green line is current.... .155

Figure 7-20. Tracking response illustrating moderate, sinusoidal speed control for $\hat{T}_{L}=1$ and $\hat{\omega}_{d}=1+0.2 \sin (2 \pi \hat{t})$. The dashed blue line is the desired speed while the solid blue line is actual speed, the red line is MR fluid thickness, and the green line is current.....156 


\section{LIST OF TABLES}

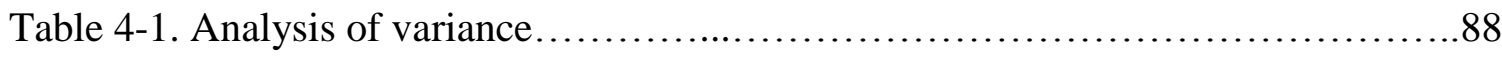

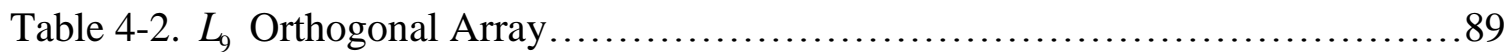

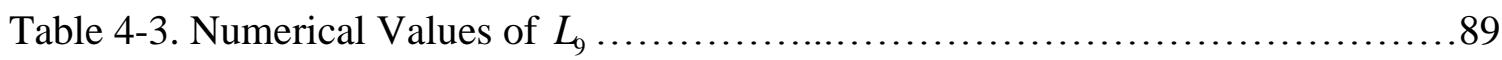

Table 4-4. Signal to noise ratio of each factors and associated levels...................89

Table 4-5. Analysis of variance for conventional frictional brake..................90

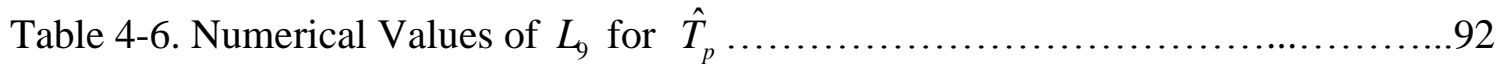

Table 4-7. Signal to noise ratio of each factors and associated levels of $\hat{T}_{p} \ldots \ldots \ldots \ldots . .93$

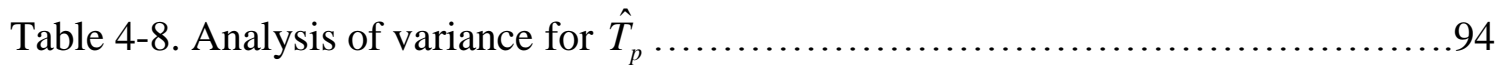

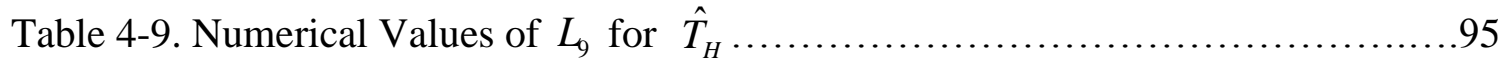

Table 4-10. Signal to noise ratio of each factors and associated levels of $\hat{T}_{H} \ldots \ldots \ldots . .96$

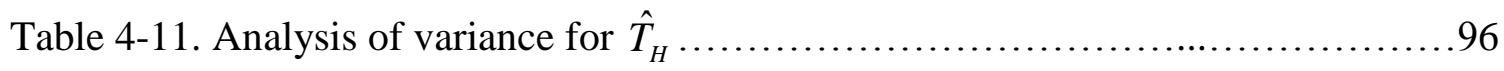

Table 5-1. Drawbacks of several magnetorheological brake designs...................105 


\section{LIST OF ABBREVIATIONS}

a linear dimension locating the applied force to the drum brake

$b$ horizontal, linear dimension locating the shoe pivot with respect to the drum center

$B \quad$ flux density

c vertical, linear dimension locating the shoe pivot with respect to the drum center

$d f \quad$ differential force on a differential elemental area

$d A \quad$ elemental area

$d r \quad$ radial coordinate

$d \theta \quad$ angular coordinate

$E_{b} \quad$ brake efficiency

F $\quad$ applied brake force

$h \quad$ instantaneous MR fluid thickness

$i \quad$ electric current

$i_{\max } \quad$ maximum applied current to the electric coil of the MR fluid brake

$J \quad$ mass moment-of-inertia for the flywheel

$\mathrm{K}_{i} \quad$ integral control gain for adjusting the MR fluid thickness

$\mathrm{K}_{p} \quad$ proportional control gain for adjusting the MR fluid thickness

$k_{w} \quad$ wire resistance

$l_{j} \quad$ magnetic field path length corresponding to section $j$

MR abbreviation for "magnetorheological"

MRF abbreviation for "magnetorheological fluid" 
$N_{s} \quad$ number of frictional surfaces

$N_{t} \quad$ number of turns of a coil

PI abbreviation for "proportional-integral" control

$P \quad$ contact pressure between the brake shoe and the drum

$P_{\max } \quad$ maximum contact pressure between the brake shoe and the drum

$R_{x} \quad$ horizontal reaction force at the pivot point of the brake shoe

$R_{y} \quad$ vertical reaction force at the pivot point of the brake shoe

$r \quad$ radius of the drum

$r_{i} \quad$ inner radius of a frictional disc

$r_{o} \quad$ outer radius of a frictional disc

$S \quad$ dimensionless sensitivity coefficient for a given dimensionless parameter

$S_{w} \quad$ wire section

$T \quad$ braking torque

$T_{L} \quad$ applied load torque

$t \quad$ time

$T_{\mu} \quad$ braking torque due MR fluid viscosity

$T_{H} \quad$ braking torque due magnetic field

$T_{p} \quad$ frictional disk brake torque using the uniform pressure theory

$T_{w} \quad$ frictional disk brake torque using the uniform wear theory

$V_{x} \quad$ a point velocity in $\mathrm{x}$-direction

$V_{r} \quad$ a rotor velocity 
$V_{s} \quad$ a stator velocity

W width of the shoe pad, into the paper

$W_{i} \quad$ thickness of section $i$

$\gamma \quad$ angular dimension of the shoe pad

$\gamma_{s} \quad$ fluid shear strain

$\dot{\gamma}_{s} \quad$ fluid shear strain rate

$\theta$ angular coordinate identifying a contact point between the shoe and the drum

$\mu_{s} \quad$ coefficient of friction between the shoe pad and the drum

$k, \beta \quad$ shear stress property for a specific MR fluid

$\varepsilon \quad$ instantaneous error

$\zeta \quad$ damping ratio

$\mu_{m M R F}$ absolute viscosity of the MR fluid

$\mu_{o} \quad$ the magnetic permeability of air gap $\left(4 \pi * 10^{-7} T . m / A\right)$

$\mu_{m} \quad$ a material magnetic permeability

$\mu_{r} \quad$ the relative magnetic permeability

$\mu_{c} \quad$ coefficient-of-friction for the frictional disk brake

$\tau \quad$ MR fluid shear-stress at the disk surface

$\tau_{H} \quad$ shear stresses due to magnetic field

$\tau_{p} \quad$ shear stresses due to magnetic field

$v$ the ratio between the applied current to the surface area

$\Phi_{i} \quad$ integral control gain for adjusting the electric current 
$\Phi_{p} \quad$ proportional control gain for adjusting the electric current

$\phi \quad$ magnetic flux

$\mathfrak{R}_{i} \quad$ magnetic reluctance of section $i$

$\omega \quad$ instantaneous shaft speed

$\omega_{d} \quad$ desired shaft speed

$\omega_{n} \quad$ undamped natural frequency 


\begin{abstract}
Mechanical and electrical brakes have dominated the braking industry for many years and will most likely continue to do so for the foreseeable future due to their low cost and adequate operating performance, wide range of applications, vehicle engineering, civil engineering, and biomedical engineering. Simple mechanical drum brake and magnetorheological (MR) fluid brake have presented in the current work. The main objective of this work is to increase braking torque, and to develop a new optimal design of MR fluid brake with better design and design control of the MR fluid design. To do so, four important steps have been accomplished. In the first step, a mathematical modeling of the conventional frictional brake and MR fluid brake has been developed to study and specify all design parameters. In the second step, a nondimensional, closedform analysis and a Taylor series expansion have used to examine the effects of perturbing dimensionless design parameters on the overall brakes performance. In the third step, two optimal designs for MR fluid brakes have been developed by taking advantage of sensitivity analysis and the design of experiments method also known as the Taguchi method. In the fourth step, controlling a MR fluid brake is performed by using two parallel PI controls for controlling the magnetic current and MR fluid thickness simultaneously.
\end{abstract}

It was concluded that sensitivity analysis is a good method for identifying the parameters that have the greatest impact on brake performance and can be used as one method for the designer to obtain an optimal design. Four nondimensional design parameters were successfully used to describe the conventional frictional brake and seven 
nondimensional design parameters for MR fluid brake. Only two parameters for the conventional brake and five parameters for the MR fluid brake affect the performance and the others can be neglected.

Two new designs for the MR fluid brake are presented and shown to be very simple in design, low in cost by removing a lot of additional auxiliaries for the frictional brake, and easy for control. By simultaneously controlling the MR fluid thickness and the electric current, a large range of brake torque is achieved without increasing the radial envelop for the brake, and saturation conditions in one controller are compensated for by the other controller. High angular velocities of the brake are primarily controlled by increasing the MR fluid thickness, while low angular velocities are primarily controlled by increasing the electric current. Good transient responses for regulating a constant speed (high, moderate, and low), and good stability while seeking to track a sinusoidal input have been achieved. In summary, the proposed control system for the MR fluid brake has demonstrated good controllability for the MR fluid brake. 


\section{CHAPTER 1. INTRODUCTION}

\subsection{Background}

Have you ever imagined that you stop a new future car by your thumb only? Smart brake and smart fluid is the key for that. This concept will be explained after introducing a general background about conventional and smart brakes and how they are developed. The car industry is dramatically developing day by day. Hundreds of car companies are investing their money and putting their products in continuous competition to develop better, cheaper, safer and higher car performance. Three important types of brakes are introduced in this section, mechanical, electrical, and Magnetorheological Fluid brake (MRF brake).

\subsubsection{Conventional and Electrical Brakes}

The story started with a conventional mechanical brake system that is used to stop an object safely. Mechanical brakes were first used to stop a horse cart, which consisted of a long lever and a big block of wood. The idea is the driver pulls a lever to contact a rotating wheel with a piece of wood attached at the end of lever providing the required retarding torque as shown in Fig. 1-1.

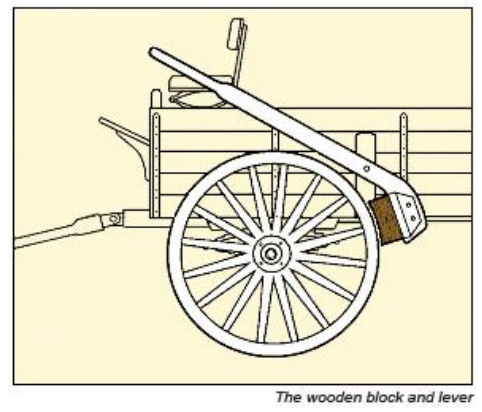

Figure 1-1. Oldest type of brake [1] 
The lever brake worked well until late 1890s and then the wood cart wheel was replaced by a rubber bobbin. However, this type of drum brake was a starting point of the mechanical drum brake. In 1899, Daimler came up with a new design and new idea which was a long cable wrapped around a drum and attached to the chassis of a moving object as shown in Fig. 1-2 [2]. His idea helped to provide a preliminary assistance force to the lever to make it easier for the driver to stop a cart.

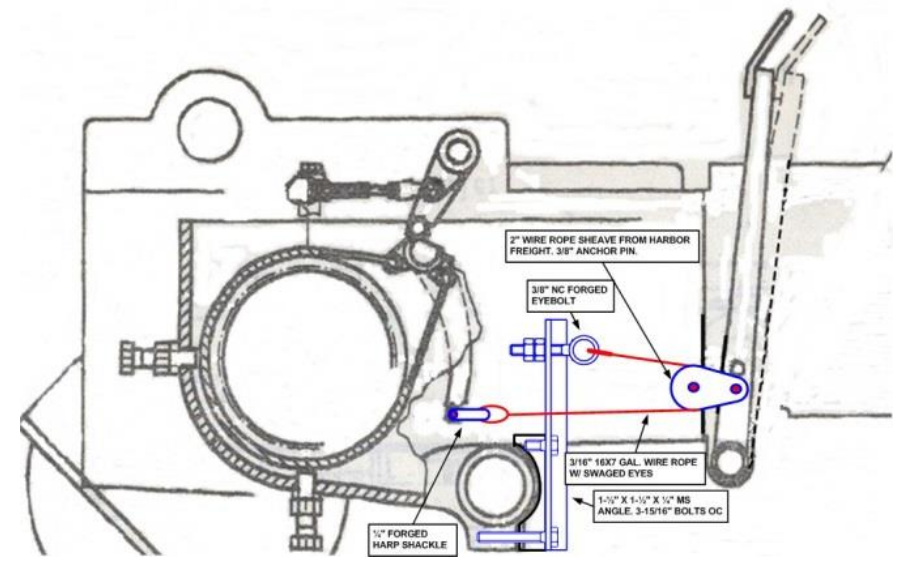

Figure 1-1. Wrapped cable brake [2]

The drum at that time was external and affected by dust and water at winter. These problems affected and reduced using lever- external drum brake. The problem was solved by using an internal drum and specific internal hydraulic pistons designed in 1918 by Malcolm Loughead. He used a brake fluid to provide the required pushing force on the brake shoe instead of the mechanical lever.

Temperature increasing affected the performance of the drum brake and became the main reason to develop a better design for the brake, which is easier for cooling. The two types of brakes, drum and disc, were developed gradually to achieve all design requirements for versatile applications. 
Figure 1-3 shows a conventional hydraulic brake system.
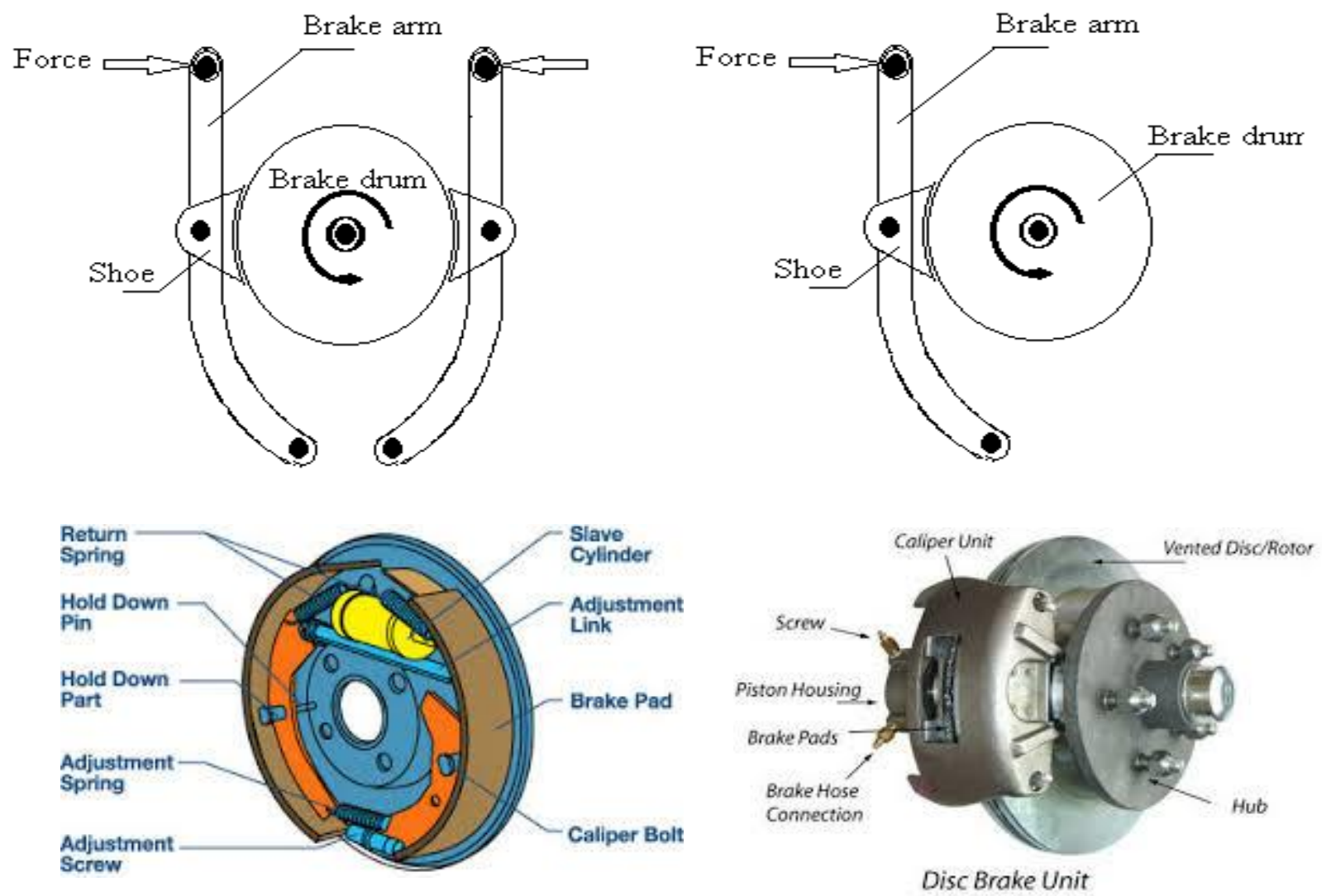

Figure 1-3. Conventional drum and disc brake [162]

The conventional brake system consists of several parts:

1- Brake pedal.

2- Power assistance system.

3- Main hydraulic pump.

4- Brake fluid and its reservoir.

5- High-pressure pipes.

6- Brake mechanism (drum or disc brake). 
The braking process can be conducted just by pressing brake pedal in order to provide the required pressure for the actuators and then provide the required normal force to the frictional surface. A simple schematic of disc brake is given in Fig. 1-4.

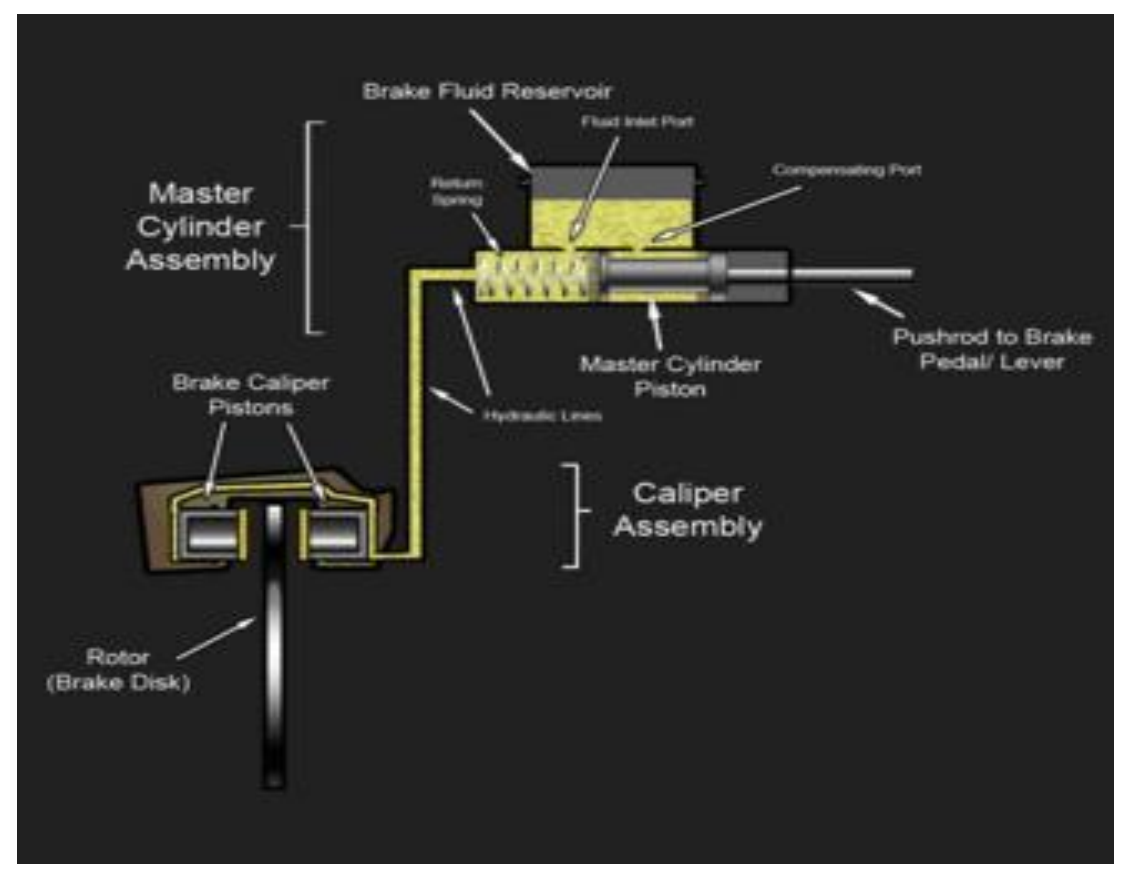

Figure 1-2. A schematic diagram for conventional brake [163]

An air brake system was created similar to the conventional hydraulic brake. The only difference between them is that the first one uses air instead of brake fluid. The air brake is used for heavy-duty brake requirements especially for large buses and trucks. In general, the conventional brake has many disadvantages:

1- The high-pressure requirements of brake fluid increase the possibility of leakage and that greatly affects the brake performance.

2- There is a time delay for pressure build up through the transfer pipe and the actuators because of the brake fluid compressibility. 
3- The conventional brake requires continuous maintenance because of the auxiliary components. It requires changing brake pads, cylinders, and drums because of wear. In order to overcome the disadvantages, an electromechanical brake has developed and applied for different industries.

This technology has been called brake by wire. In this kind of brakes, electrical components have added to the mechanical parts of the conventional brake in order to increase the brake efficiency and the performance. Several companies have started using electromechanical actuators instead of the hydraulic one. Delphi Company has used electromechanical brake in their products [3]. Another type of brakes is just appeared called an eddy brake. However, the eddy type cannot be considered as an efficient brake and cannot be used alone because of its low performance. The eddy brake works like a dc generator by converting the mechanical energy into electrical energy $[4,5]$.

\subsubsection{Magnetorheological Fluid Brake}

Within the huge developments of brake by wire technology and the developed electrical controllers, another development has introduced into brake design, Magnetorheological Fluid brake (MRF brake) as shown in Fig.s 1-5 and 1-6.

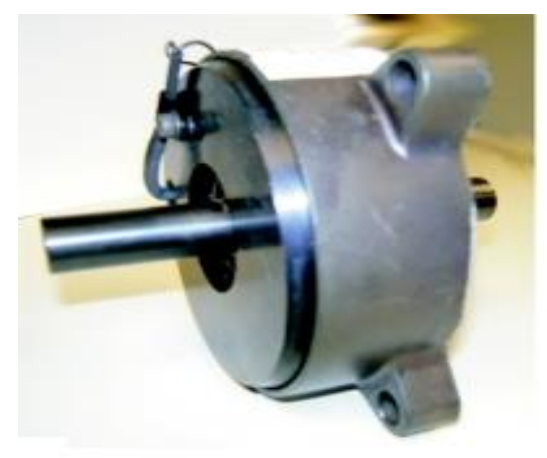

Figure 1-5. A typical magnetorheological [164] 


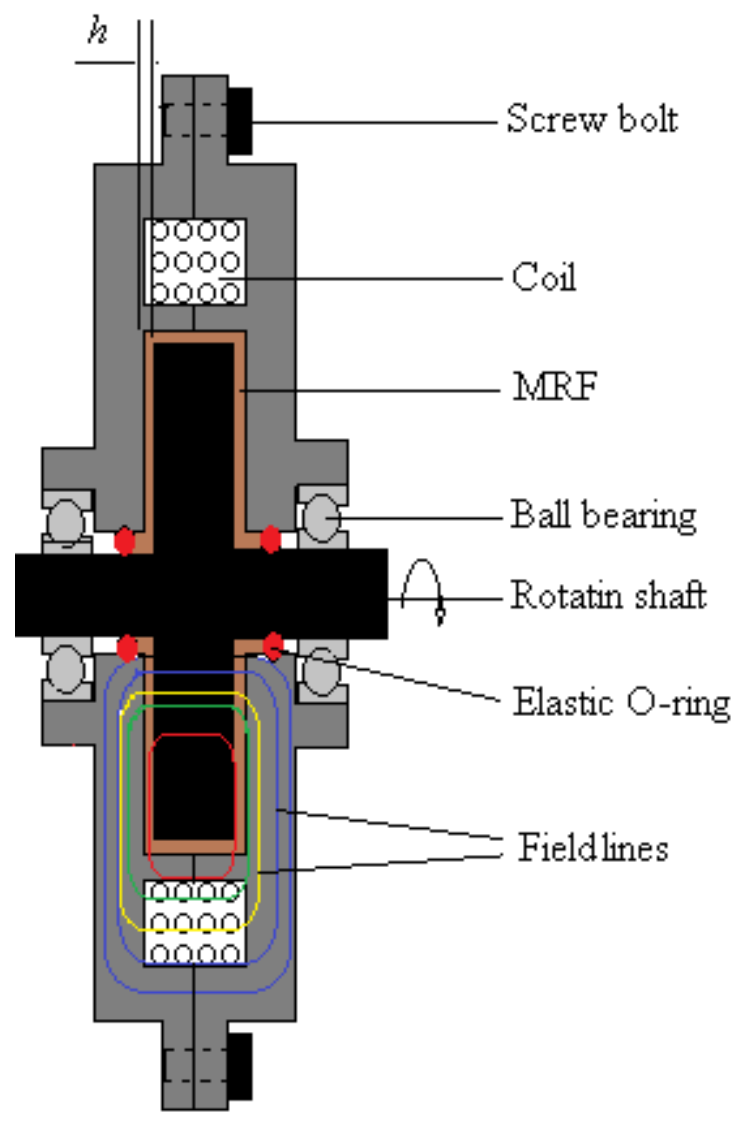

Figure 1-6. A schematic diagram of a typical magnetorheological fluid brake

A typical magnetorheological fluid brake consists of several parts:

1- Screw to fasten the whole assemply of brake cases.

2- Rotaing shaft that carry the rotating disc.

3- Ball bearings in order to suupport the rotating shaft.

4- Magnetorheological Fluid (MRF).

5- An elctrcial coil in order to create the required magnetic filed.

6- An elastic O-rings to prevent fluid leakage.

The magnetorheological fluid brake has more advantages than other types, for example:

1- A quick brake response. 
2- Easy to manage and control.

3- No brake pads and smaller contact surfaces.

4- Little auxiliary components, no brake pedal, no power assistance system, no hydraulic pump, no brake fluid and reservoir, no environmental effect, and no high-pressure pipes.

5- Braking process can be conducted just by using a small controller to control an input current to a magnetic coil. Braking mechanism is going to be explained in detail after getting a general background about magnetorheological fluid, its physical properties, and its response in the presence of magnetic field.

\subsection{Magnetorheological Fluid History and Background}

Some researchers called MRF brake as a smart brake that because of using a smart fluid. Smart fluid or responsive magnetic fluid can be defined as a fluid that can be controlled under the effect of external factors. Jacob Rabinow, who worked at the National Bureau of Standard (NBS) at the United states, created this kind of fluid as a patent in 1948 [87]. Magnetorheological fluid (MRF) can be easily defined as a fluid that changes its viscosity when exposed to a magnetic field. It mainly consists of two phases; nano-magnetically permeable particles (typically from 1 to 5 microns) immersed in a special carrier fluid $[6,7,18,19]$. In the normal conditions (absent of the magnetic field), the nano-particles are just suspended and randomly distributed throughout a carrier fluid. However, these particles rearrange themselves in high stiffness chain like-structure along lines of the magnetic field [33] as shown in Fig. 1-7. These chains like-structure are very strong. Chain stiffness increases the apparent viscosity of the magnetorheological 
fluid and changes the fluid from a liquid (off-magnetic field) into a solid (on-magnetic field) thereby increasing the apparent magnetorheological fluid viscosity.
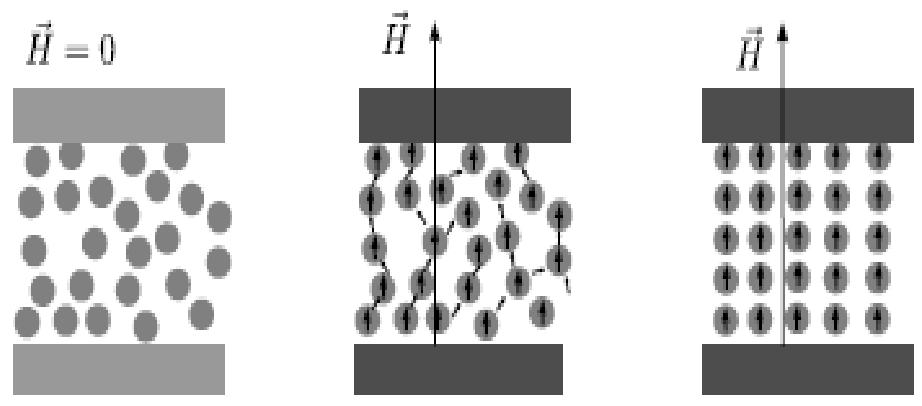

Figure 1-7. Chain like-structure of a magnetorheological fluid due to applied the magnetic field [33]

The repeated processes of creating and breaking these chains is the main source of the force that resists fluid motion. Fluid resistivity for the shearing stress increases with the applied magnetic field [50]. The larger the magnetic field, the stronger the resistive forces against fluid flow. Magnetorheological fluids have typical characteristics and can be illustrated as:

1. Of the most important characteristics of MRF are their very fast response in changing their state from a liquid to a solid, which basically has a linear relationship with the magnetic field. That reduces the time response of all devices working within a magnetorheological fluid.

2. A quick reversibility of its state (depending on presence of magnetic field), which can be done very quickly within one millisecond.

3. Wide ranges of working conditions. For example, almost all magnetorheological fluids are thermally stable $\left(-40 C^{o}\right.$ to $\left.150 C^{o}\right)$ [60]. 
4. High level of controllability in changing its state from a solid to a fluid and vice-versa by simply controlling the applied magnetic field.

Oil fluids like hydrocarbon oil [146], silicone oil [147] are widely used as carrier fluids with carbonyl iron nano-particles. The main reason of using iron particles is their higher saturation level which about 2-2.1 Tesla [ 148]. The presence of the resistive forces due to off/on magnetic field is the main source of the frictional torque of MRF brake.

\subsection{Physical Composition of a Magnetorheological Fluid}

A magnetorheological fluid is basically consisting of two phases, magnetic particles and carrier fluid. The magnetic particles could be iron particles, nitride particles, iron carbide particles, or carbonyl [149-151]. Each of these magnetic particles presents a different response under action of same magnetic field. That response depends on two important magnetic characteristics, particle magnetic saturation and particle coercivity. Coercivity can be defined as the magnetic field intensity required to demagnetize a material (reduce its magnetization to zero) after material magnetization is driven to the saturation [149]. Based on that, particles have higher magnetic saturation are preferred in the magnetorheological fluid applications. Magnetic saturation and coercivity is explained in detail in Chapter 5. Carbonyl particles and iron particles are mostly used [152].

\subsection{Magnetorheological Fluid in Applications}

Many companies have worked on magnetorheological fluid brake and used it for great application. For example, it used for shock absorber and seismic applications as 
shown in Fig. 1-8 [10-13], haptic device [14], clutch and brake [15, 16], and prosthetic devices [17].

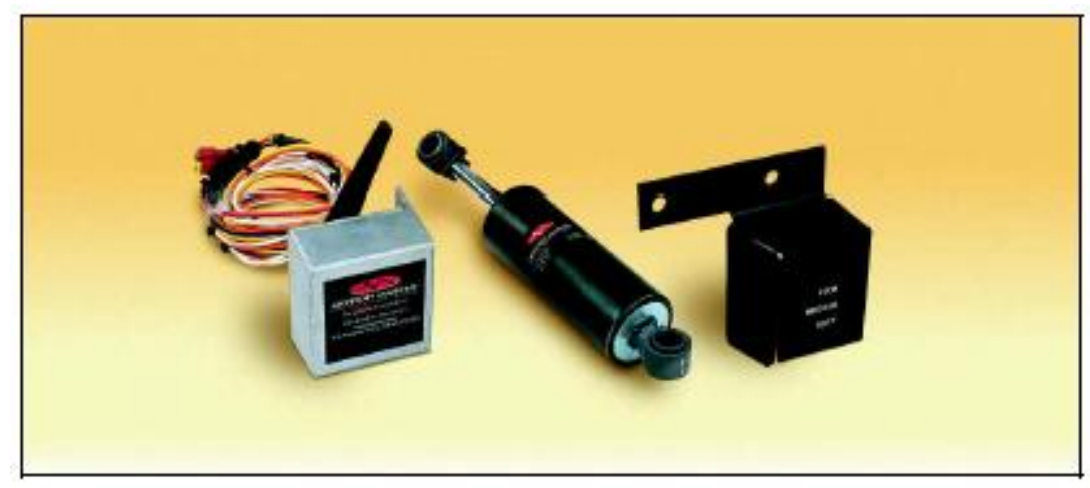

(a). Seismic application [13]

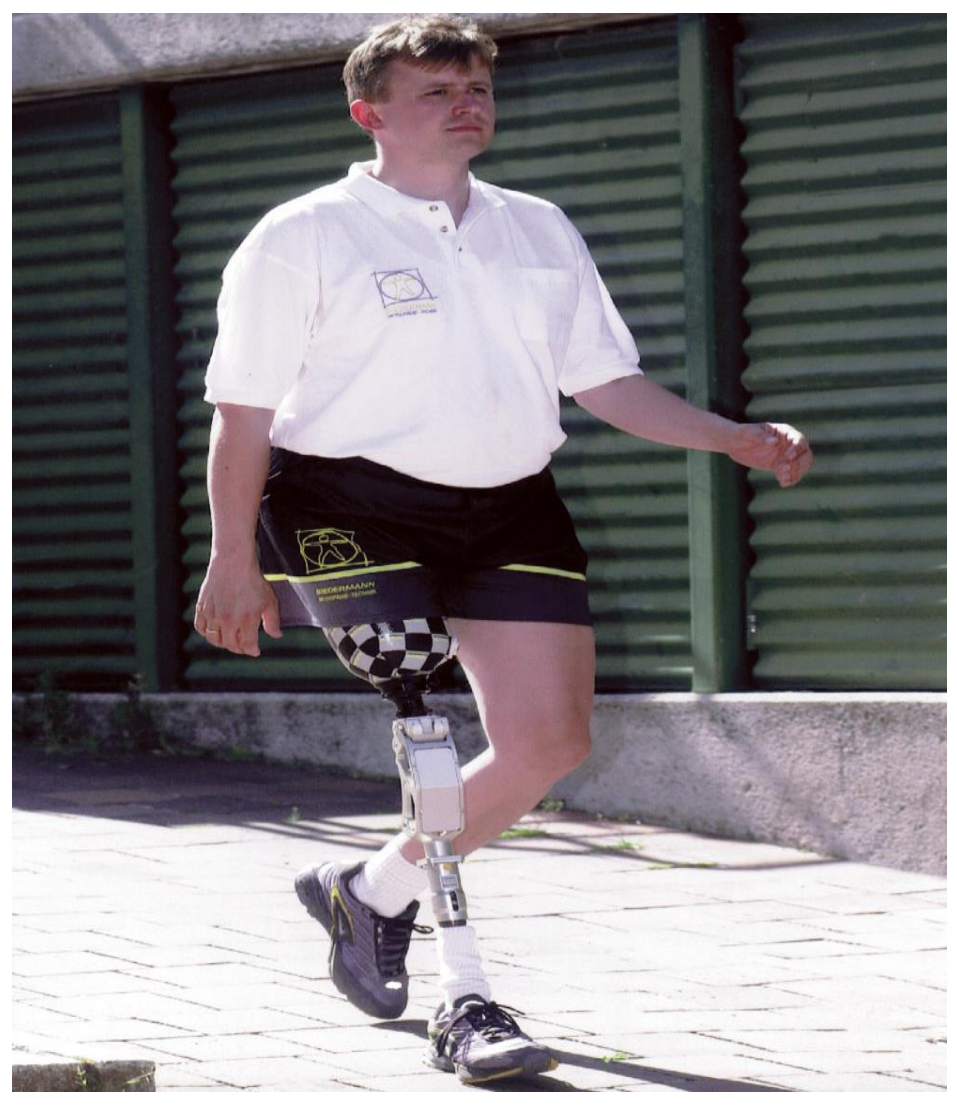

(b). Prosthetic applications [17] 

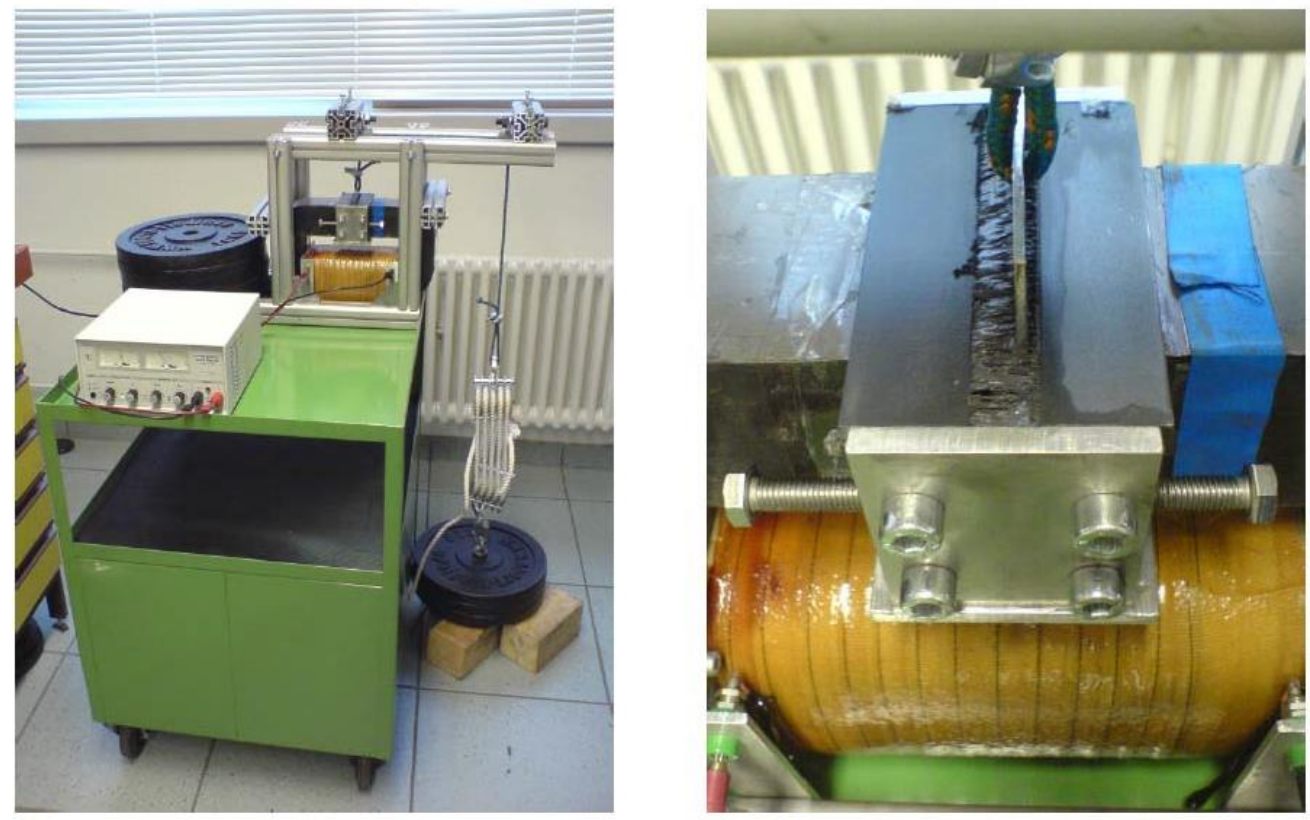

(C). Fixture for complex shape applications [79].

Figure 1-8. Magnetorheological fluid application for seismic and prosthetic devices

Another important usage of the magnetorheological fluids, that appeared in literature, is a fixturing device during part machining. People used to put a magnetorheological fluid in a specific location that a specimen (which is not easy to fix using conventional method because of shape complexity) required to be fixed $[18,79]$. Another interesting application of the magnetorheological fluids is in a casting (screw copy process). Rainbow [154] mentioned a very good way to use a magnetorheological fluid to create a bolt screw by copying. A bolt is immersed in a magnetorheological fluid reservoir just in the normal conditions (no magnetic field is applied). After that, a magnetic field is enforced to pass through the fluid changing its state form liquid to solid or may be partially solid depending on how the process requires. With a presence of magnetic field, bolt is loosen leaving exactly same screw profile. 


\subsection{Dissertation Objectives}

The main objectives of this dissertation are:

1- To develop a simple mathematical modeling of a single drum brake and a magnetorheological (MR) fluid brake.

2- To conduct a perturbation analysis using Taylor series to learn how design parameters affect brake performance and define which of design parameters have the greatest impact.

3- To use sensitivity analysis to obtain an optimal design of a magnetorheological fluid brake by changing geometrical brake design for one or two effective design parameters.

4- To use the Taguchi method to obtain an optimal brake design for specific limits of design parameters.

5- To conduct a parametric study to explain effect of several electrical parameters on MRF brake response such as magnetic impedance, magnetic reluctance, and permeability.

6- To control a MR fluid brake using PI control design to show weather MR fluid brake controllable or not.

7- To compare the torque capacity between the conventional frictional brake and the MR fluid brake. This is presented in the Appendix.

\subsection{Dissertation Outline}

The current work is organized in seven chapters. A general and comprehensive background is presented in the present chapter in order to explain some important 
characteristics of magnetorheological fluid and principle of altering its viscosity. The reminder chapters of the dissertation are described as follows:

Chapter 2. In this chapter, a general review of what has been done by other people is presented as a literature review. It is presented in three parts: analysis and optimal design of magnetorheological fluid brake, magneto-rheological fluid brake, and magnetorheological fluids behavior. A summary for this chapter is presented as well.

Chapter 3. This chapter introduces a general theoretical background about drum and MRF brakes including the basic principles of working modes of the fluid, fluid shear stress and strain, drum and the magnetorheological fluid brake parts and free body diagram, governing equations, electrical and magnetic considerations. Non-dimensional analysis of drum and a typical MRF brake to make the mathematical modeling much more general is presented as well.

Chapter 4. This chapter presents a general introduction about design of the experiments using the Taguchi method, Taguchi approach, orthogonal array, quality loss functions, signal to noise ratio, and analysis of variance. The main aim of this chapter is using Taguchi method to obtain an optimal design for both a single shoe drum brake and magnetorheological fluid brake for a specified limits and design parameters ranges.

Chapter 5. This chapter presents a general background about the old design of magnetorheological fluid brake and its drawbacks. Two main problems of the electromagnetic circuit, magnetic saturation and hysteresis loop are presented. Two new deigns of magnetorheological fluid brakes are developed which are called the continuous variable torque and variable fluid thickness brakes (CVFT1 $h$ and CVFT2 $h$ ). A general 
procedure beyond getting the new designs taking the advantages of sensitivity analysis is showed as well.

Chapter 6. This chapter introduces a control design for the MR fluid brake using PI control. In this chapter the principle of work for the suggested CVFTs MR fluid brakes and how they are connected to the actuator is showed in detail. MRF brake control includes a description for the proposed MR fluid brake, dynamic analysis of the flywheel needed to be controlled is illustrated. Nondimensional analysis is carried out to generalize the model and make it more efficient. Finally, using linearization the control gain selection is clearly illustrated.

Chapter 7. Results and discussions of the previous brake analyses under specific consideration are presented in this chapter. The effect of changing several parameters are going to be discussed in detail as well.

Chapter 8. This chapter presents some highlight conclusions by combining whole thesis in one package and mention some important suggestions for the future work. 


\section{CHAPTER 2. LITERATURE REVIEW}

\subsection{General}

To present a good summary about some important up to date researches for conventional and magnetorheological fluid brake, an extensive literature review has been done. The current literature review gives a historical background and an outline about how conventional and magnetorheological fluid brake have developed. Regarding to the current research, a literature review has been completed including three main parts. The first part has included sensitivity analyses for the conventional brake. The second part has included sensitivity analysis and magnetorheological fluid brake, its modeling, and an optimal design. The third part has included several researches about magnetorheological fluid properties and fluid behavior models. Several keywords have been used to conduct a search for literature on this subject using Scopus; for example, sensitivity analysis, conventional drum brake, magnetorheological fluid brake, and magnetorheological fluid properties. To my knowledge, the literature presented here is the most up to date.

\subsection{Sensitivity Analysis and Conventional Brake}

Manring, N. D., \& Mehta, V. S [19], used sensitivity analysis to find design parameters, which have the greatest impact on the bandwidth frequency of a pressure controlled axial-piston pump. Their theoretical study was motivated based on the physical limitations of this machine that have been observed in practice. The complexity of the model was subsequently reduced by using nondimensional analysis, which eliminates negligible terms. They also presented a closed form expression for the bandwidth frequency. To identify a set of parameters that affect the bandwidth 
frequency, they used sensitivity and perturbation analyses. The researchers concluded that there are set of important parameters impact the overall response of the machine. The nondimensional groups from sensitivity analysis gave a good insight about pump performance.

Manring, N. D. [20], studied a sensitivity analysis of a conical shaped equivalent model of a bolted joint. The governing equations describe the bolted joint model were made non-dimensional using nondimensional groups. Relative importance to estimate the proper model and deign parameters based upon the sensitivity analysis was also presented. Washer diameter, bolt length to diameter ratio, and cone angle were included in the sensitivity analysis. Furthermore, error and maximum percent of uncertainty of the spring-rate calculations was also showed. The author concluded that the sensitivity analysis was very important and gave good prediction about important design parameters and estimation of an accurate model.

Pannell, D. J. [21] showed very useful information about sensitivity analysis, methods, and concepts in addition to some examples. He defined the sensitivity analysis as an investigation of potential changes and errors resulted from parameter values and assumptions of any model. Different approaches to sensitivity analyses and overall strategies were also showed. The best way to sum up the implications for the model, understanding the overall behavior of system response, and how the system parameters affect each other were also showed. The researcher concluded that even without deeply pointing out the complex relationship between system parameters and their impact on the system, sensitivity analysis is a powerful and illuminating method. Furthermore, sensitivity analysis of the simple approach is easy to do, to understand, and communicate. In addition, the author pointed out that just a simple approach to sensitivity analysis presented the best method to take advantage of whole 
understanding of the system parameters, thereby, particular decision making.

Ostermeyer, G. P [22], studied effect of wear and its importance on a conventional brake model. A new type of second order differential equation for the dynamic friction coefficient to describe the stationary and transient friction behavior of brake pad was suggested. Principal wear mechanism, which takes equilibrium of flow of growing and destruction of a hard patching, was adopted. Some known important parameters of friction like fading effect and periodic change of dynamic friction coefficient were also presented. The researcher concluded that wear played an important role and had a great effect on the friction dynamic behavior. Furthermore, friction coefficient affects brake torque. Dynamic friction coefficient decreased when disc velocity increased. However, he did not mention to what extent is going to be effect of the friction coefficient on brake torque.

Othaganont, P. et al [23], achieved sensitivity analyses of an automotive powertrain. A method, based on formal optimization mathematics for second ordersensitivity analyses, was analytically developed. This method was applied on a case study of C-segment electrical car. Beside their new method, the researchers applied the first order sensitivity analysis as well. It was concluded that the first order sensitivity analysis gave good information about which parameter has the great effect on the model response. While, the second order sensitivity analysis gave an insight about the relationship between effective parameters and the interaction coupling between the parameters and whether that coupling is weak or strong. Finally, the researcher stated that sensitivity analyses used in his research were the keys to obtain the optimal design of his model.

Tortorelli, D. A., \& Michaleris, P. [24], presented a design finite difference sensitivity analysis with its application to linear systems. First and second order 
sensitivity analysis for different examples were derived. A simple two degrees of freedom dynamic system was adopted to illustrate effect of masses and springs (as design parameters) on its eigenvalues and eigenvectors. The researcher concluded that sensitivity analysis plays an important role in the inverse and identification study especially those deals with an optimization filed. However, some disadvantages of finite difference sensitivity analysis appeared and affect the overall results due to truncation and round of error.

Kucherenko, S. et al. [25], used a sensitivity analysis to identify effective dimensions of a specific mathematical model. Their main idea was to find effective parameters or effective dimensions rather than dealing with all design parameters (which make the model much more complicated) and then fix those of less effective importance at their nominal values. The researchers used the global sensitivity analysis based on ANOVA (Analysis of Variance) which measure the variations of output variables due to the variation of input variables. They divided the functions of multiple input variables into three parts: functions with not equally important variables, functions with equally importance variables, and functions with equally importance variable but with dominant interaction between them. They concluded that sensitivity analysis is superior methodology and can easily reduce the complexity of any mathematical model.

Hwang, I. J., \& Park, G. J. [26], studied judder phenomenon (a steering wheel vibrates abnormally at high speed during brake) using both mode and sensitivity analyses. They mentioned two important ways to eliminate this phenomenon; modifying the disc shape and optimizing the suspension system. In this research, they improved the suspension system. Mode analysis using finite element method was accomplished to find vibration frequencies for all parts of the system. To analyze the 
phenomenon, a real vehicle was utilized. They remodeled and modified the tested car suspension system by reworked bush system. They finally pointed out that sensitivity analysis and its results were the keys to understand how suspension system affected judder phenomenon and why bush system was the most important design parameter.

Anstett Collin, et al. [27], studied a sensitivity analysis for a complex mathematical model for both static and dynamic inputs variables. In this research, dynamic inputs are considered as random processes. The input variables then were defined using finite number of random variables. Depending on that definition, they used sensitivity indices of these variables to define its variance based sensitivity indices. The prescribed mathematical approach was applied to a building energy model. More than fifty-six static and dynamic variables were used as input variables. They concluded that only four inputs of these variables were significant for predicting the energy for that building. Sensitivity analysis helped them to decide which of these variables have a maximum and pronounced effect on the system response.

Martins, J. R. R. A. et al. [28], created an automated method for sensitivity analysis using complex variables. They based on the idea that the complex number is just one number even though it consists of two parts (imaginary and real). They showed the difference between the direct differentiation using finite difference, automatic differentiation, and an analytical method. To verify the validity of their automated method, two examples were presented. The first one was for a structural sensitivity of a finite element model of a wing (stress sensitivity to the change in the wing cross section) and the second one was for an aerodynamic sensitivity (change of angle of attack and Mach number) of that wing using CFD. It was concluded that the predicted methods were more implementation advantageous over automatic differentiation and more computational advantageous over finite differencing method 
because it is less sensitive to the step size.

Yang, C. P., \& Liu, T. [29], illustrated a new mechanical design to solve the problem of brake locking for two-wheel bicycles. Mechanism of the system and its principle of working were presented. A mathematical model for effected forces and torque were also showed. Effect of several important parameters such as friction coefficient (an indication for a wet, dry, and iced road), device orientation angle, values of the attached springs and its effective free length were studied in detail. It was concluded that the new device has advantages and disadvantages in the same time. It can be useful to reduce or eliminate the brake locking whatever the magnitude of applied force is, but that has a negative effect on the brake performance (because of the fixed pad force). Another drawback of this device is that each application should have special and suitable design parameters different than other one. In other words, the device is not self-adjustable for every design.

Rocha, A. B., \& Silveira, Z. C. [30], presented a conceptual design for a disc brake and its application for hybrid car. The major point of their research was energy transform from mechanical to heat transferred inside the disc. Uniform pressure and uniform wear brake theories were used for the mathematical modeling. Effect of several important parameters affect the generated temperature was studied. It was noted that design variables like diameter and pad materials in addition to the normal force and the required torque have a great effect on the temperature gradient. There were good agreements between the present results and that corresponding in the literature.

2.3 Analysis and Optimal Design of Magnetorheological Fluid Brake Huang, J. et al. [31], presented a design method for a cylindrical magneto- 
rheological fluid brake system. They relied on the Bingham representation of the fluid to describe the relationship between shear stress and shear rate. Brake torque, which is depending on the yield stress developed by a magnetic field, was mathematically derived. Radius, width, thickness, and the minimum volume of the magnetorheological fluid of the brake system were considered as effective parameters in the derived model.

Park, E. J et al. [32], developed a magnetorheological fluid brake system. It mainly consists of two rotating discs included inside a magnetorheological fluid and an electromagnet as a source of an electromagnetic filed. ANSYS finite element simulation was used to find the optimum design of the brake system. In the finite element method, magnetic flux distribution, fluid flow, temperature distribution, and heat transfer phenomenon of the suggested brake system were considered. In order to control the wheel slip, a slide mode controller was designed to keep a slip ratio at its desired values and make a compromise between the maximum frictional force and the stopping distance. It was concluded that there are some advantages for their new brake design as compared with the conventional hydraulic system like quick response and low weight besides being environmental friendly. In addition, brake evaluation and its response showed good results regarding anti-lock braking at different friction conditions of dry and ice roads.

Rossa, C. et al. [33], developed a wide-ranged- magnetorheological fluid brake. Magnetic flux density and its distribution through fluid gab were obtained using ANSYS. The main aim of their work was specifying important design parameters that affect the desired output of the brake (torque). They defined their developed design using three parameters; fluid gab numbers, radius, and gab thickness. Finite element method was used to find the optimum design of the 
proposed brake geometry. To verify their mathematical model, an experiment was achieved to find the torque output. The results showed good agreement with $5.5 \%$ relative error.

Sohn, J. W. et al. [34], conducted an experimental evaluation of a magnetorheological fluid brake. The proposed design was a disc type and suitable for mid-sized motorcycle. Herschel-Bulkley rheological model was used for torque calculations. To get better design, increase brake torque, reduce the mass and working temperature of magnetorheological fluid, gradient descent algorithm optimization method with ANSYS 12 was conducted. Mechanical performance and time response were experimentally evaluated. It was noted that MRF brake was very efficient as compared to the conventional hydraulic brake, but MRF brake was heavier than that corresponding of conventional one.

Bigué, J. P. L. et al [35], studied the super-strong phenomenon of a magnetorheological fluid compared with Péclet number. They defined the superstrong effect in terms of torque to weight ratio. Their test bench provides a small shear and squeeze oscillations. Many conclusions are drawn from their work. The main conclusion was the super-strong effect and squeeze strengthening could be correlated and related to Péclet number. They used this number to make three distinct results regards super-strong effect. If $\mathrm{P}>>1$, magnetorheological fluid behaves as a typical fluid, if $\mathrm{P}<<1$ then MRF reorganized its phases distribution and phases filtration might be occurred, but if $\mathrm{P}=1$, phase transition begins and shear strength increases gradually. Furthermore, they introduced another important factor, $\mathrm{n}$, which is the ratio of squeeze rate to shear rate. They end up with, the super-strong effect was significantly hindered when this factor almost equal unity.

Shiao, Y., \& Nguyen, Q. A [36], presented a new design for MRF brake in 
order to enhance braking torque. They depended on some modifications in magnetic field strength. Multiple electromagnetic poles surrounded by several coils were used in their new design. These poles were arranged in a specific way to increase active fluid regions. The primary design was assumed and then optimized using Sequential Nonlinear Programming (SLNP) incorporated with finite element method. Effect of different design parameters, input current, poles gap, MR layer thickness, MRF type, and steel properties, was presented. It was noted that there was a specific poles gap at which output torque got its maximum value. MRF layer thickness and the torque had an inverse relationship. AISI 1018 carbon steel gave maximum torque compared to other steel types. Finally, 140CG MR fluid presented maximum torque to input current ratio.

Rossa, C. et al [37], discussed design considerations for magnetorheological brakes. New terms were defined and used to define and evaluate the brake performance; torque density, efficiency, and reactivity. They considered two important models, disc brake and drum brake models. ANSYS finite element analysis was used to find magnetic field distribution along fluid gab. They showed that the reactivity of the drum brake is more dominant than disc brake type for same torque to weight ratio. Brake efficiency decreased with gab increasing. Brake radius increases the maximum torque and decreases the efficiency.

Phu, D. X. et al [38], created a new design of engine mount for horizontal and vertical control using magnetorheological fluid brake. Their design was more advantageous than conventional mount because it offered higher efficiency to mount dimension. The primary design was assumed and an optimum design (which is simply achieved by increasing gab fluid numbers) was achieved in order to increase torque output and damping force in both horizontal and vertical directions. The proposed 
design was designed based on the general idea of MR fluid brake incorporated with the main rubber mount type. An experimental validation was accomplished to verify the analytical results. It was noted that there were small differences between them. In addition, the new mount decreased the excitation displacement and velocity to $98 \%$ and $79 \%$, respectively.

Nguyen, Q. H. et al [39], proposed a new design of magnetorheological brake with integrated built in coils. The magnetic coils were wounded inside a specific rounded groove created in the brake main housing. Bingham plastic model was used for the mathematical modeling to calculate the output torque. The initial design variables (geometrical dimensions) were specified and optimized to maximize braking torque and reduce brake mass and volume. Finite element method was used to accomplish an optimization processes. Rectangular and polygon shapes for the brake housing were considered in the main design. The optimized design was verified and tested experimentally. It was concluded that the optimized brake with integrated coils was much lighter than the conventional design. Furthermore, the new design was compact and smaller in its volume, then easy for manufacturing, assembling, and low in unit cost. However, the problem of temperature is just appeared.

Nguyen, Q. H. et al [40], conducted an optimum design of different magnetorheological brake types to reduce the mass and maximize the torque. Disc, Tshaped, and drum brake were considered throughout that research. Herschel-Bulkley model was adopted for mathematical modeling and torque calculations. The primarily design variables were specified and optimized using finite element program (ANSYS). Optimal power consumption was also calculated for brake types. It was demonstrated that disc and T-shape brake types were the best in terms of compactness. However, it consumed much power than other types. Drum brake was 
the worst because of its large size.

Russo, R., \& Terzo, M. [41], designed an adaptive control for a magnetorheological fluid brake tacking temperature and speed effects into the considerations. Their study was experimentally and theoretically executed. Bingham plastic model was used for the mathematical modeling of the viscosity and magnetic field effects. Effects of temperature and rotational speed and their contribution on the output torque were presented. Some important parameters such as time constant and torque/current gain were studied as well. An adaptive control approach was designed to ensure convergence of the tracking error to its minimum value (zero) when time increase. It was noted that contribution of the magnetic field decreased with the rotational speed while viscosity contribution decreased with temperature.

Nguyen, Q. H., \& Choi, S. B. [42], obtained an optimal design for different types of magnetorheological fluid brakes such as drum type, disc type, hybrid type, inverted drum type, and T-shaped type. Herschel- Bulkley model was used for the mathematical modeling of the viscosity and magnetic field effects. The optimization processes (which included increasing number of coils and changing of disc and drum shape) were considered to obtain the maximum torque with constrained volume. Torque ratio (ratio of the maximum torque to the one of zero magnetic fields) was assumed to be constant. Finite element method was used to find the magnetic field distribution through brake body. Optimum designs of all types were given as a function of radius to length ratio. Experiments were conducted to validate the theoretical modeling. The results showed good agreements between them.

Park, E. J. et al. [43], developed an electromechanical brake system using magnetorheological fluid. The main idea was using MRF to enhance a retarding torque with a presence of magnetic field. The developed brake was mainly consisting 
of a disc immersed in a magnetorheological fluid. Changing the applied current to produce a specific magnetic field was the main way to control the developed brake. ANSYS was used as a tool for brake analysis. An optimization study was performed to reduce the brake weight under specific constraint. Two different magnetorheological fluids were used as working fluids for one and two discs brake, respectively. They concluded that the developed brake can meet the common requirements of the conventional hydraulic brake at smaller weight.

Nguyen, Q. H. et al [44], studied the optimum design of magnetorheological brake tacking the change in the brake case envelope in the considerations. Three different types of case envelope were studied, rectangular, five segments and seven segments, and spline envelopes. The braking torque output was calculated based in Bingham theory of plastic behavior of a magnetorheological fluid. The optimization procedure aimed mainly to find the best configuration that gives the best magnetic field distribution and minimum brake weigh. ANSYS finite element analysis was used for the optimization purposes. It was concluded the configuration played an important role regarding the brake performance. The seven segments configuration gives the best results regarding torque and magnetic field distribution. No significant difference between the seven segments and spline configuration was noticed.

Song, B. K. et al [45], developed a new design for magnetorheological fluid brake to study effect of bobbin materials on brake performance. In his modified design, the coil wire is wounded on the brake case directly without using nonmagnetic bobbin material. Bingham model is used for the mathematical modeling of the fluid behavior. The bobbin configuration was redesigned in a way such that the magnetic flux is prevented to pass through the fluid duct and to prevent the bottle-neck of the magnetic field phenomenon. Braking torque and braking force were calculated 
theoretically and verified experimentally. For the theoretical part, ANSYS finite element package within first order method was used. Golden section method was used for the optimization purpose as well. It was stated that using of magnetic bobbin materials instead of the conventional design (nonmagnetic one) gave good performance characteristics. Number of coil windings and corresponding consumed electrical energy are reduced. In addition, the manufacturing of magnetic bobbin became much easier, thereby lesser cost.

Nguyen, Q. H., \& Choi, S. B. [46], studied an optimal design of a magnetorheological brake for a middle car size tacking both geometric dimensions and zero-filed friction into account. Both Bingham and Herschel-Bulkley rheological models were used in the mathematical modeling. A good comparison was done to find their effect on the results. Temperature effect due to repeated brake usage was taken into the consideration as well. Three models of brake were studied, single, double, and triple rotary discs. Three different types of the working fluid (low, medium, and high yield stress) were used in the analysis as well. ANSYS finite element was used for the analysis and optimum design under constraint functions. Herschel-Bulkley rheological models are more accurate than Bingham. However, Bingham model gave good results.

Choi, S. B. et al [47], used an electro-rheological brake system for speed control of a DC motor. The brake used for this purpose was consists of two concentric cylinders separated by a gab in between. The outer cylinder is fixed while the inner cylinder was connected to a DC motor to control its speed. Bingham model was used in the mathematical modeling of the fluid behavior. To achieve the requirements of the output signal and tracking it in good manner, sliding mode control algorithm was used. The response was calculated for different cases. Tracking error was calculated 
as well and compared with a conventional PID controller. It was clearly noted that the slide mode controller gave better results than PID regarding the tracking error. Electro-rheological brake showed very good results and it could be used for control purpose as well.

Nguyen, P. B., \& Choi, S. B. [48], proposed a new approach for analysis a magnetic circuit used for a magnetorheological brake. Bi-directional magnetorheological brake was developed to provide a positive and negative braking torque. The main idea of the proposed circuit analysis was based upon changing coil locations and then discretizes the brake domain into several segments. Ampere's and Gauss's laws were then used to find the relationship between the magnetic density and magnetic flux for each segment. Beside the new approach of electrical circuit, Bingham plastic model for fluid is used. Maxwell finite element analyses software was used to find the optimum design of the proposed brake. Experimental study to verify the accuracy of the results was conducted as well. Comparison of the results showed several advantages of the proposed analysis on using finite element method. In addition, the computational time was very short as compare to finite element method.

Erol, O. G. et al [49], suggested an optimization method to find the optimal design of a small actuator to achieve maximum torque to volume ratio. Effect of several important parameters was taken into calculations. Finite element analysis was used to find out magnetic flux distribution through the MRF. Fluid yield stress was calculated at each step of optimization processes. The geometrical variables were incorporated directly in the suggested optimization method, but other variables such as electromagnetic current was interacted with the finite element package to find its effect on the yield stress. The main feature of this method was the reduction in 
computational time for getting the optimal solution. A prototype was also prepared and the results were compared. A small discrepancy was noticed due to experimental errors.

Rossa, C. et al [50], suggested a novel design for torque detection of a magnetorheological actuator. They presented a simple mathematical model using elementary magnetic reluctance theory for a ferromagnetic particle immersed in a carrier fluid. Two coils were used; one to produce the required magnetic field and the second to measure the induced impedance resulted from the relative displacement between the particles. They concluded that the presented technique is very useful for torque release detection and it might be used as an indication of chain-like structure rupture.

Assadsangabi, B. et al [51], designed and accomplished an optimal design of a magnetorheological fluid using genetic algorithm. The initial geometrical design parameters were first adjusted to find the corresponding mathematical model. Then ANSYS finite element was used to find the magnetic field distribution along with the fluid gab. The main goal of the optimization was to find maximum torque to weight ratio. After successive iterations of the optimized design, they concluded that the final design was larger in the volume than corresponding from other designs and did not fit to use in automobile brake.

Polcar, P. [52], built a prototype for a simple magnetorheological fluid brake of low torque output in order to understand the brake mechanism experimentally and theoretically. His model consisted of one apparent disc immersed directly in magnetorheological fluid. Agros2D finite element software was used to find a flux magnetic field distribution. It was noted that the fluid gab and the supplied current have a great effect on the output torque. Torque responded linearly when the current 
is low until a critical current value. After a critical current, the torque increases dramatically. In addition, the mainly disadvantage of this brake is the off-state torque resulted from the fluid viscosity without magnetic field.

Perniata, A. N. R. S. et al [53], developed a drum type magnetorheological fluid brake. Brake design was easily consisted of T- drum part that with two faces of frictions. ANSYS Maxwell finite element package was used to find a flux density distribution along the drum body. Effect of current on the magnetic flux, brake torque, and the steady state time (to reduce the drum velocity to zero) was studied. It was concluded that the settling time reduce with increasing the applied current and instantaneously increases torque output.

Ma, L. et al [54], designed a magnetorheological fluid brake used for an electric passenger car. The initial design parameters were assumed and corresponding torque was calculated based on it. Bingham plastic model was used for the mathematical model. Effect of magnetic field and viscosity of the fluid was studied with and without magnetic field. Finite element method was used to find the optimum design of the proposed design considering speed, geometrical design parameters, and the magnetic circuit. It was concluded that the brake torque increased with the supplied current.

Sukhwani, V. K., \& Hirani, H. [55], described design characteristics and design procedure for a magnetorheological fluid brake. Effect of fluid gab was studied by design two brake prototypes of $1 \mathrm{~mm}$ and $2 \mathrm{~mm}$, respectively. Three magnetic coils were installed. One of them placed in the center and the other two placed on both side. A simple mathematical model tacking Bingham plastic model was presented. It was concluded that, brake torque decreased with fluid gap before magnetic field saturation for all ranges of current used. However, the torque response showed different 
response after saturation. Braking torque increased with increase the magnetic field.

Ma, L. et al. [56], designed a multiple disc type- magnetorheological fluid brake to meet real requirements of passenger car. Ten discs were used in the presented design. High magnetic field and shear speed were considered in the design and the mathematical modeling. Magnetic field distribution was calculated using finite element method and considered in calculation of shear stress at specific points. It was concluded that off-field brake torque (no magnetic field) increased with speed and cannot be neglected. $90 \mathrm{~W}$ and $600 \mathrm{~W}$ were applied to achieve $600 \mathrm{Nm}$ and $1000 \mathrm{Nm}$ torque to meet the real requirements of passenger car.

\subsection{Magneto-Rheological Fluid Brake and Fluid Behavior}

Yang, L. et al [57], presented a general overview about analysis and design of magnetorheological fluid structures. The researchers paid an attention about all parameters and variables that may affect design of a smart structure. Beside effect of the physical properties of the fluid which related to increase or decrease the fluid yield strength, they considered other parameters from other sources such as magnetic circuit parameters, fluid volume fraction, and power losses parameters. For the magnetic and circuit analysis, COMSOL finite element analysis was used to find magnetic field distribution throughout the brake body. This research does not consider temperature effect on the fluid properties. They concluded that the optimum design of such smart structures should consider all the geometric parameters of the brake fluid and, electrical circuit parameters.

Jolly, M. R. et al, [58], presented a suggested mathematical model to describe the behavior of magnetorheological fluids. Saturation and nonlinear magnetic field distribution were considered in their modeling. Bingham plastic model was used to 
describe the fluid behavior. Pre-yield (an elastic response) and post-yield response (viscous response) of the magnetorheological fluid were assumed in the mathematical modeling and experimentally confirmed. Several parameters such as volume fraction and particle reluctance were studied to find its effect on the fluid yield strain. The analytical model was compared to an experiment to find correction factors resulted from curve fitting of the most important effective parameters (yield stress and change in fluid modulus). It was stated that particles volume fraction played a key role regarding fluid behavior. Fluid modulus increased with particles volume fraction up to $30 \%$, for example. Finally, they showed that the suggested model and the assumption made were reasonable as compared with experiments.

Wereley, N. M., \& Pang, L. [59], developed nonlinear semi-active electrorheological and magnetorheological dampers (line friction) using Bingham plastic model. Three fluid modes were used; shear mode, flow mode, and mixed mode to calculate the damping force. In addition, a nondimensional study to assess the mathematical model was done. Three non-dimensional groups, Bingham number, nondimensional plug number, and bypass cross sectional area were identified to get an insight about how the model is sensitive for a specific disturbance. Damping coefficient for the three shear models were considered and then verified experimentally. It was concluded that damping force was linear function for Bingham number and nondimensional plug thickness for shear and flow modes, respectively.

Chen, S. et al [60], studied effect of temperature change on both magnetorheological fluid properties on a transmission system performance. The main concentration was how magnetorheological fluid viscosity was sensitive to a temperature difference ( -40 to $150 \mathrm{C}^{0}$ ) with and without a magnetic field. Reynolds equation for viscosity temperature dependent was used in their formulation. Herschel- 
Bulkley plastic model to define the relationship between shear strain and shear stress was used as well. It was stated that the temperature has a noticeable effect on the fluid viscosity, shear stress, and then the transmission torque. The rate of temperature effect varied along with a specific temperature range. In other words, at very low and very high temperature, the viscosity increased gradually. The major conclusion of their work was that: once the temperature changes, the transmission torque is no longer controllable.

Wang, D. et al [61], studied effect of temperature on several parameters of a magnetorheological fluid such as magnetization properties, shear viscosity, and thermal expansion. It was found that the magnetization properties and shear viscosity decreased with temperature while the thermal expansion increased. Fluid of higher shear viscosity showed more sensitivity to the temperature change than that corresponding of lower value. However, thermal expansion showed a little sensitivity to the temperature change for the magnetorheological fluid. They recommended to use a fluid of low shear viscosity to enhance a thermal stability and good sealing system must be used to prevent fluid sealing due to thermal expansion and fluid volume change.

Kim, M., \& Park, S. J. [62], presented a mathematical model to find effect of several parameters on a magnetorheological fluid (magnetic particles dispersed in silicone oil). Effects of temperature, shear rate, volume fraction, and magnetic flux were studied. Magnetorheological fluid was tested experimentally for seven values of volume fraction, three temperature values, and ten magnetic flux values. Results of each test under specific variable values were plotted against shear strain, and then effect of each variable was represented by an equation with some constants. It was noted that the fluid viscosity decreased continuously with the shear rate, decreased 
with temperature, and increased with both volume fraction and magnetic flux. They proved that there is a saturation magnetic flux after which the viscosity did not show further increase.

Patil, S. R. et al [63], studied transient thermal analysis for a magnetorheological fluid brake. The analysis was accomplished for an e-bicycle magnetorheological fluid brake. The main goal was studying the temperature distribution and temperature rise in the brake assembly. They considered that the temperature was generated by both fluid shear stress and power losses in the electrical circuits due to the resistance. ANSYS finite element package was used to find the temperature distribution in the brake at different boundary conditions. Based on their results, they concluded that temperature of the fluid under those conditions stayed within the working range of magnetorheological fluid. The analysis was experimentally verified and they found good agreements between both results.

$\mathrm{Li}$ and Zhang [64], studied a friction effect on magnetorheological fluid properties under effect of both steady and dynamic shear rate states. Experimental analysis was used to perform this task. Different shear rates were investigated and frictional force was measured. It was concluded that both frictional force and magnetic force affected magnetorheological fluid properties. They pointed out that friction factor played an important role in identifying and enhancing fluid properties. Shear stress increased with friction factor. In addition, they proved that shear stress increased almost linearly with shear strain for both steady and dynamic states.

Wang, D. M. et al [65], developed a high torque magnetorheological brake and used water for cooling and heat dissipation. He used multi-plate brake design as a good criterion to increase number of frictional surfaces and then increase the braking torque. Bingham plastic assumption was used for plastic model. Simple mathematical 
model for both temperature sources of fluid slip and electrical components were presented as well. An experimental study was conducted to understand effect of temperature, water flow rate, and input current. Temperature has a direct impact on the resulted brake torque as a function of time. Using water as working fluid for cooling was a good choice.

\subsection{Summary}

Several closing remarks can be concluded from overall literature review. The concluding remarks are very helpful and some of them can be adopted in the current research because they are already verified and no further proof is required: 1) The researchers who worked on or used sensitivity analysis stated that sensitivity analysis was very helpful to get an insight about how design parameters affect design performance and how they interact one each other. For example, [19, 20, 21] concluded that even without deeply understand the relationship between system variables and far from the complex relationship between system parameters and their effect on the system, sensitivity analysis is still a powerful and illuminating method. Furthermore, sensitivity analysis using the Taylor series is a simple approach, easy to do, understand, and communicate. In addition, they pointed out that just the simple approach is the best method to take advantage of the whole understanding of the system parameters, 2) That the sensitivity analysis played an important role in the inverse and an identification study especially those dealing with an optimization field because it helps to estimate the more significant design parameters that affect design performance [24], 3) That sensitivity analysis using ANOVA is superior in identifying the variance of output variables due to variance of input variables [25]. Then, the variance method could be incorporated with sensitivity analysis to find an optimum 
design of the MRF brake, 4) Most researchers support using the Bingham plastic model in their mathematical modeling of MRF brakes [31, 39, 41, 44-48, 54, 55, 58, $59,65]$. Therefore, the Bingham plastic model will be adopted through the current research. Some of them used Herschel-Bulkley model and they showed that there is no significant difference between the two models and the first still works well [34, 40, 42, 46, 60], 5).Reference [50] throughout his study about MRF behavior showed that shear stress of MRF can be increased during fluid compression between two poles. This is a very important conclusion and can be adopted in current research to create a new MRF brake design using fluid compression concept to enhance shear stress and then increase brake torque, 6) Some researchers have considered their optimum design by only increasing the number of surfaces of contact or by decreasing fluid gap thickness. In both cases, a high value of off-field torque or viscous torque can be built up and further power should be consumed [38, 65, 53, and 56]. Therefore, one can make a tradeoff between gap number and off-field torque to get better performance, 7) Almost all of brake optimization methods appeared in the literature review had been done either by using finite element method or by simply changing one of brake parameters. For example, changing brake envelope, increasing number of gab, increasing number of coils, etc. These methods and techniques actually leave the researcher out of play and most time he does not know how optimization technique works and even how the design parameters of MRF interact each other, and 8) to our knowledge, no one has worked on sensitivity analysis of MRF brake or studied how design parameters changes affect MRF brake performance, controlling the magnetorheological fluid brake using current and fluid thickness, and comparison study between magnetorheological fluid brake and the conventional frictional brake. The results presented in this dissertation will be the first of their kind. 


\section{CHAPTER 3. MATHEMATICAL MODELING AND ANALYSIS}

\subsection{Introduction}

In the first and the second chapters, a general background and literature reviews have been presented to get an idea about conventional and magnetorheological brake and what other research has been performed on these devices. In this chapter, we are going to present the mathematical modeling for both mechanical and magnetorheological fluid (MRF) brake. The basic question needed to be answered is whether braking torque for conventional and magnetorheological fluid brakes can be physically described using a set of nondimensional groups, and whether or not perturbation analysis may be used to find the nondimensional groups that have the greatest influence on braking torque. To do that, this section is divided into three parts. The first and second parts have answered the first part of the research question regarding to the conventional brake and magnetorheological fluid brake using sensitivity analysis, respectively. However, the third part presents a new suggested design for the MRF brake based upon the simple mathematical model.

\subsection{Sensitivity Analysis of a Single Shoe Conventional Drum Brake}

\subsubsection{General}

A conventional brake is considered in this section. In order to understand and check if the hypothesis of using sensitivity analysis can be applied for magnetorheological brake, a simple case of a conventional mechanical drum brake has been studied because drum brakes have dominated the braking industry for many years and will most likely continue to do so for the foreseeable future due to their low cost and 
adequate operating performance. Basic equations for designing these brakes have been presented in college textbooks, while complicated analysis has been published using finite element methods to predict brake squeal and instability. This study seeks to step away from the complexity of numerical models to consider the fundamental braking phenomenon of a single-shoe drum brake, using nondimensional, closed-form analysis and a Taylor series expansion to examine the effects of perturbing dimensionless design parameters. Braking systems are ubiquitous within mechanical systems for providing safety for both dynamic and static loading conditions. The drum brake has served for many years as the dominant brake design, compared to say disc-brake designs, for both industrial and automotive systems due to a low acquisition and operating cost, and satisfactory braking performance for most applications. While college textbooks contain classic equations and design procedures for drum brakes [66-72] these texts do not generally address the subject of brake optimization. Furthermore, these texts present the equations in dimensional form without elucidating the fundamental physics of the brake design by identifying nondimensional groups that are responsible for creating the brake function. Although nondimensional studies have not been performed to the authors' knowledge, other dimensional research has been conducted to optimize the design of drum brakes. For instance, Sayim and Zhang have conducted dimensional analysis and experiments to show that the drum brake is able to achieve comparable performance to that of a disc brake [73]. These results were important, but being dimensionally dependent they were not generalized for all brake designs. Huang, et. al., have sought to identify the onset of braking instability in order to capture brake squeal [74]. In their research, they put forward a method for identifying a critical mode of operation using 
finite element analysis, while also suggesting a perturbation technique for studying design sensitivities that are able to address brake squeal. Again, this was a very useful contribution emphasizing a method of analysis, but stopped short of making generalized conclusions for all drum brakes of a given design. Nechak and colleagues have also studied the problem of brake squeal, suggesting the use of Kriging models for estimating brake vibration, which is an alternative to using the finite element methods [75]. In their work, these researchers have conducted sensitivity analysis based on nondimensional Sobel indices which identify the coefficient of friction as the dominant contributor to brake instability. Other examples of using finite element analysis to study the performance of drum brakes have existed in the literature for many years [76-78].

\subsubsection{Analysis and Brake Description}

A schematic of the single-shoe drum brake is shown in Fig. 3-1. In this figure, the shoe is shown by the cross-hatched element and is attached to a lever arm that pivots about a pivot point from which the dimensions $a, b$ and $c$ are referenced. The brake drum is the circular element beneath the shoe and is shown to rotate in the counter clockwise direction with a load torque $T$ applied to the drum by a driving element not shown in Fig. 3-2. In order to resist the applied torque acting on the drum, a friction torque is applied to the drum by the input force $F$ which generates a pressure between the shoe and the drum, thus producing the friction torque for stopping or slowing the speed of the drum. In Figure 3-2, the geometry of the shoe is shown by the angular dimension $\gamma$ which is centered symmetrically above the drum as shown. In the following analysis, the free-body-diagrams for the shoe and for the drum will be considered in order 
to develop an equation for the break torque applied to the drum.

\subsubsection{Free-Body-Diagram Analysis}

Figure 3-3 shows the free-body-diagram for the shoe. In this figure, the drum has been removed and replaced by an infinitesimal reaction force in the direction normal to the drum shown by $d f$, and an infinitesimal friction force shown by $\mu_{s} d f$ which acts on the shoe in a direction tangential to the drum and in the direction of drum rotation. By summing forces on the shoe in the horizontal direction and setting them equal to zero, the following equation for static equilibrium may be written

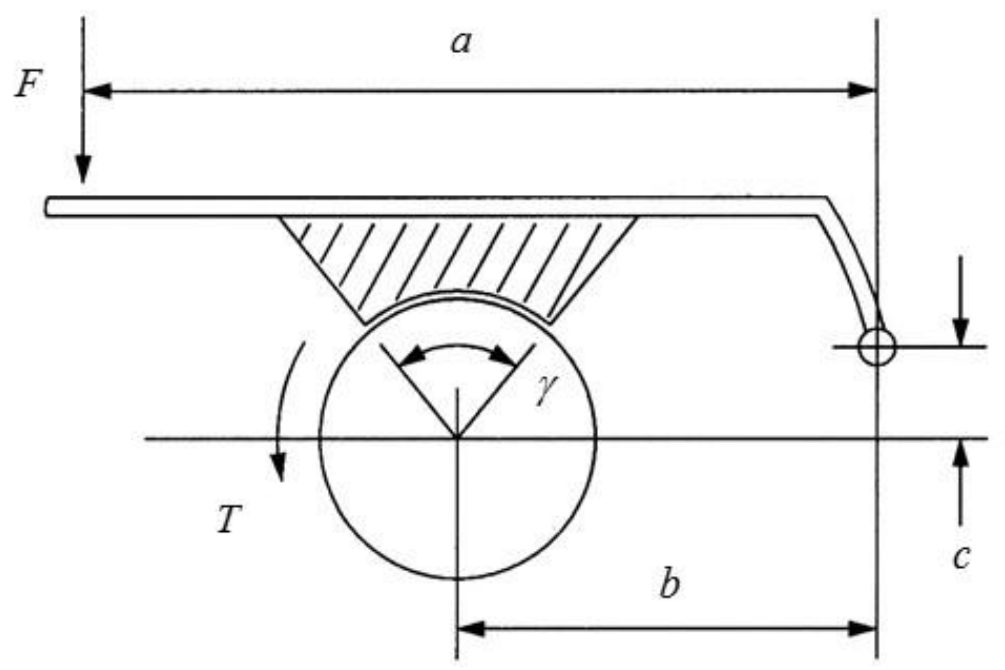

Figure 3-1. A schematic of the single-shoe drum brake

$$
0=R_{x}+\int_{(\pi-\gamma) / 2}^{(\pi+\gamma) / 2} \cos (\theta) d f-\int_{(\pi-\gamma) / 2}^{(\pi+\gamma) / 2} \sin (\theta) \mu_{s} d f
$$

In this equation, the infinitesimal contact force between the drum and the shoe is given by 
the relationship.

$$
d f=\operatorname{Pr} W d \theta
$$

where $P$ is the local contact pressure or stress between the brake and the drum, $r$ is the radius of the drum, and $W$ is the width of the shoe into the paper. For long-shoe brake designs, this pressure is generally modeled as [66-72]

$$
P=P_{\text {max }} \sin (\theta)
$$

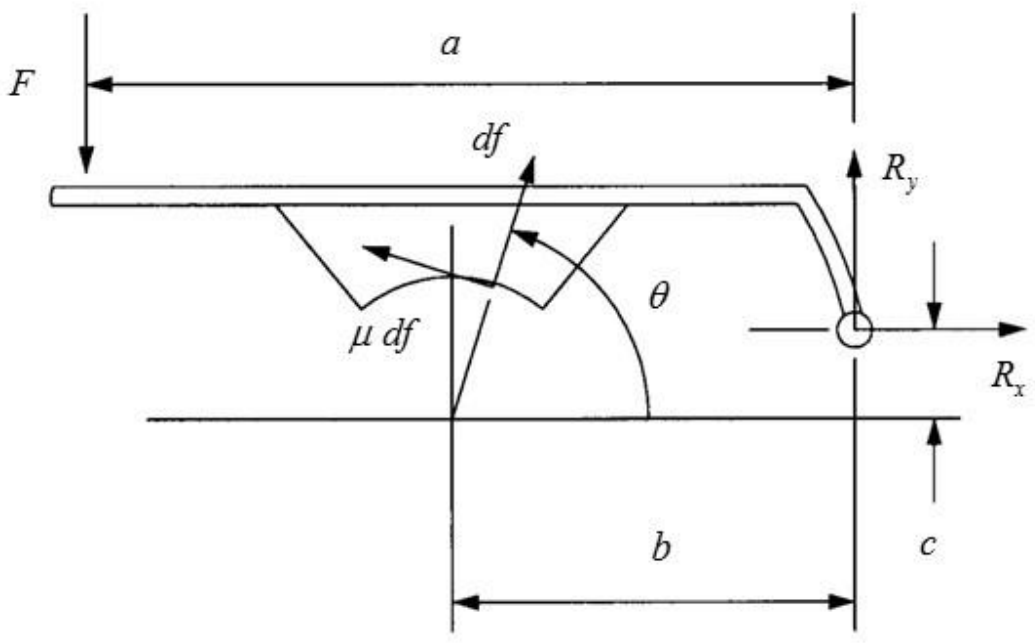

Figure 3-2. Free-body-diagram for the shoe

where $P_{\max }$ is the maximum stress between the drum and the shoe and its magnitude generally depends upon the applied force to the shoe. The material properties of the brake shoe will have a limit that must be observed in practice for $P_{\max }$. This will be discussed later. For the analysis that follows, we will assume that we are operating below that limit and the brake materials undergo no fracture or malfunction during the braking operation. Substituting Eq.s (3.2) and (3.3) into Eq. (3.1) produces the following result 
for the horizontal reaction force,

$$
R_{x}=\mu_{s} P_{\max } r W \frac{\gamma+\sin (\gamma)}{2}
$$

Similarly, by summing forces on the shoe in the vertical direction and setting them equal to zero, the following equation for static equilibrium may be written:

$$
0=R_{y}+\int_{(\pi-\gamma) / 2}^{(\pi+\gamma) / 2} \sin (\theta) d f+\int_{(\pi-\gamma) / 2}^{(\pi+\gamma) / 2} \cos (\theta) \mu_{s} d f-F
$$

Substituting Eq.s (3.2) and (3.3) into Eq. (3.5) produces the following result for the vertical reaction force:

$$
R_{y}=F-P_{\max } r W \frac{\gamma+\sin (\gamma)}{2} .
$$

Summing moments on the shoe about the center point of the drum and setting them equal to zero produces a third equation for static equilibrium:

$$
0=F(a-b)+R_{y} b-R_{x} c+\int_{(\pi-\gamma) / 2}^{(\pi+\gamma) / 2} r \mu_{s} d f
$$

Substituting the previous equations into this result and rearranging terms yields the following result for the maximum pressure that exists between the shoe and the drum:

$$
P_{\max }=\frac{F a}{r W\left[\frac{\gamma+\sin (\gamma)}{2}(b+\mu c)-2 \sin \left(\frac{\gamma}{2}\right) \mu_{s} r\right]} \text {. }
$$

Figure 3-3 shows the free-body-diagram for the drum. In this figure, the infinitesimal force $d f$ is shown to be applied on the drum in an equal and opposite direction as compared to Fig. 3-2 which shows this force being applied to the shoe. Summing moments about the center of the drum and setting them equal to zero produces 


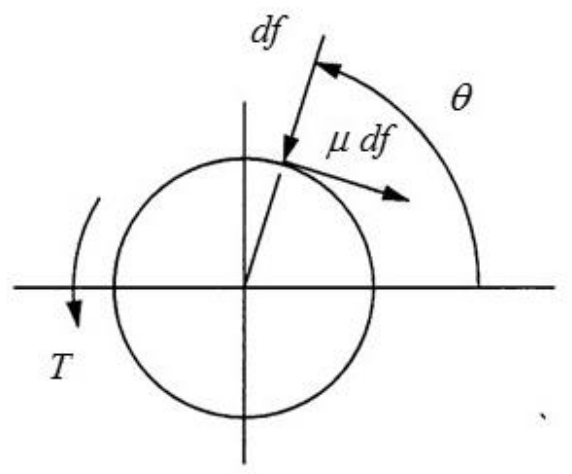

Figure 3-3. Free-body-diagram for the drum

the following equation for the braking torque of the drum brake:

$$
T=\int_{(\pi-\gamma) / 2}^{(\pi+\gamma) / 2} r \mu_{s} d f=2 P_{\max } r^{2} W \mu_{s} \sin \left(\frac{\gamma}{2}\right)
$$

Substituting Eq. (3.8) into Eq. (3.9) produces the following result for the braking torque of the drum brake:

$$
T=\frac{F a}{\left[\frac{\gamma+\sin (\gamma)}{4 \sin (\gamma / 2)}\left(\frac{b}{r \mu_{s}}+\frac{c}{r}\right)-1\right]} .
$$

\subsubsection{Nondimensional Form of Conventional Brake}

In order to conduct the sensitivity analysis that follows, the torque shown in Eq.

(3.10) will be nondimensionalized using the following definitions:

$P_{\max }=\hat{P}_{\max } \frac{F}{r w \mu_{s}} \quad, \quad T=\hat{T} F r \quad, \quad a=\hat{a} r \quad, \quad b=\hat{b} r \mu_{s} \quad$ and $\quad c=\hat{c} r$.

In this equation, the symbols with carets are nondimensional. Substituting Eq. (3.11) into Eq.s (3.8) and (3.10) produces the following expressions for the nondimensional 
pressure between the brake pad and the drum, and the nondimensional braking torque:

$$
\hat{P}_{\max }=\frac{\hat{a}}{\left[\frac{\gamma+\sin (\gamma)}{2}(\hat{b}+\hat{c})-2 \sin (\gamma / 2)\right]} \text { and } \hat{T}=\frac{\hat{a}}{\left[\frac{\gamma+\sin (\gamma)}{4 \sin (\gamma / 2)}(\hat{b}+\hat{c})-1\right]}
$$

From this equation, it may be seen that the braking torque depends upon only four nondimensional design parameters: $\hat{a}, \hat{b}, \hat{c}$ and $\gamma$. In the following section, the Taylor series will be used to conduct sensitivity analysis for the purposes of identifying the most sensitive parameters that can be adjusted in order to alter the physical characteristics of the brake.

\subsubsection{Sensitivity Studies}

The sensitivity analysis for the braking torque, applied to the drum brake, will be conducted using a first order Taylor series expansion for the torque equation presented in Eq. (3.12).

This Taylor series expression is;

$$
\hat{T}=\hat{T}_{o}+\left.\frac{\partial \hat{T}}{\partial \hat{a}}\right|_{o}\left(\hat{a}-\hat{a}_{o}\right)+\left.\frac{\partial \hat{T}}{\partial \hat{b}}\right|_{o}\left(\hat{b}-\hat{b}_{o}\right)+\left.\frac{\partial \hat{T}}{\partial \hat{c}}\right|_{o}\left(\hat{c}-\hat{c}_{o}\right)+\left.\frac{\partial \hat{T}}{\partial \gamma}\right|_{o}\left(\gamma-\gamma_{o}\right)
$$

This result can be rearranged and written as

$$
\left(\frac{\hat{T}}{\hat{T}_{o}}-1\right)=\underbrace{\frac{\partial \hat{T}}{\partial \hat{a}} \frac{\hat{a}}{\left.\hat{T}\right|_{o}}}_{S_{\hat{a}}}\left(\frac{\hat{a}}{\hat{a}_{o}}-1\right)+\underbrace{\left.\frac{\partial \hat{T} \hat{k}}{\partial \hat{b}} \frac{\hat{b}}{\hat{T}}\right|_{o}}_{S_{\hat{b}}}\left(\frac{\hat{b}}{\hat{b}_{o}}-1\right)+\underbrace{\left.\frac{\partial \hat{T}}{\partial \hat{c}} \frac{\hat{c}}{\hat{T}}\right|_{o}}_{S_{\hat{c}}}\left(\frac{\hat{c}}{\hat{c}_{o}}-1\right)+\underbrace{\frac{\partial \hat{T}}{\partial \gamma} \frac{\gamma}{\left.\hat{T}\right|_{o}}}_{S_{\gamma}}\left(\frac{\gamma}{\gamma_{o}}-1\right),
$$

where the sensitivity coefficients are given by 


$$
\begin{aligned}
& S_{\hat{a}}=1, S_{\hat{b}}=\frac{-1}{1+\left[\hat{c}-\frac{4 \sin (\gamma / 2)}{(\gamma+\sin (\gamma))}\right] \frac{1}{\hat{b}}}, S_{\hat{c}}=\frac{-1}{1+\left[\hat{b}-\frac{4 \sin (\gamma / 2)}{(\gamma+\sin (\gamma))}\right] \frac{1}{\hat{c}}} \\
& \text { and } S_{\gamma}=\frac{\gamma\left(1+\frac{\hat{c}}{\hat{b}}\right) \cos (\gamma / 2)(\gamma-\sin (\gamma))}{2\left\{1+\left[\hat{c}-\frac{4 \sin (\gamma / 2)}{(\gamma+\sin (\gamma))}\right] \frac{1}{\hat{b}}\right\} \sin (\gamma / 2)(\gamma+\sin (\gamma / 2))} .
\end{aligned}
$$

These coefficients may be used to determine the largest impact of changing either $\hat{a}, \hat{b}$, $\hat{c}$ or $\gamma$. In other words, the sensitivity coefficient with the large absolute value will indicate that a plus or minus percent change in that parameter will create the largest impact on the torque capacity of the drum brake.

\subsubsection{Summary}

In this section, the mathematical model of conventional drum brake is presented. Dimensionless analysis is used in order to make mathematical modeling much more general and working regardless the system units. With the help of Taylor series, a sensitivity analysis has conducted for the nondimensional braking torque in order to understand effect of design variables of drum brake on braking torque using sensitivity coefficients.

\subsection{Sensitivity Analysis of Magnetorheological Fluid Brake (MRF)}

\subsubsection{General}

The second important part of the current work can be divided into two parts. The first one presents several basic definitions regarding MRF, some physical properties, 
modes of operations, analysis and design, and a mathematical modeling of a typical MRF brake with some of relevant equations that state its function and principle of working. Basic definitions about magnetic circuit design are presents as well. The second part shows sensitivity analysis using perturbation method. Advantage of studying sensitivity analysis and its results may be used to work on a new suggested design of MRF brake as well.

\subsubsection{Magnetorheology}

MRF can be easily defined as a fluid that change its viscosity when exposes to a magnetic field, it mainly consists of two phases, nano-magnetically particles (typically from 1 to 5 microns) are immersed in a special carrier fluid [6, 7, 35]. Magnetorheological fluids are expressed as active and smart fluids. The apparent viscosity increases when the fluid exposed to a magnetic field by which the nanoparticles change their arrangement to create like chain structure in the direction of the applied magnetic field lines as shown in Figure 3-4.

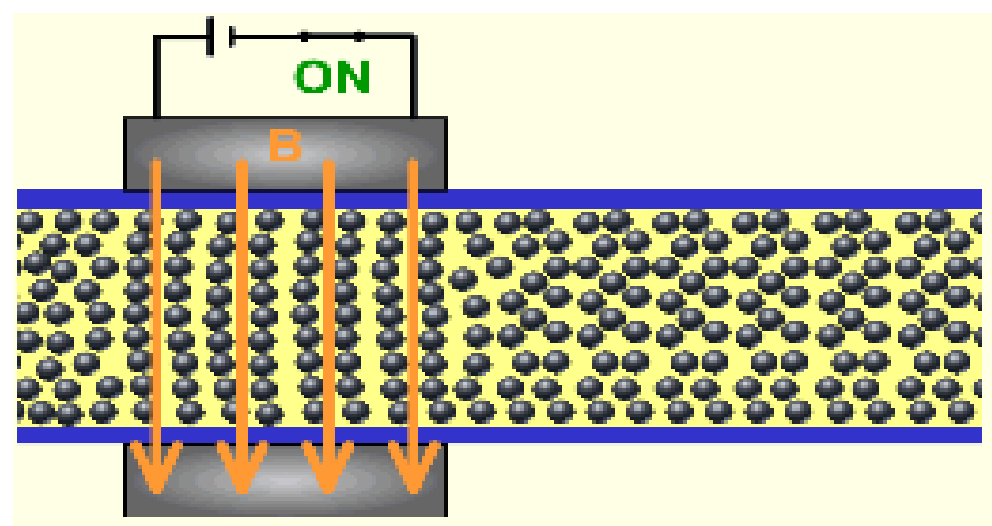

Figure 3-4. Schematic of a magnetorheological fluid solidifying and blocking a pipe in response to an external magnetic field [80] 
This like chain structure is very strong [9]. Fluid resistivity for shearing stress increases with increasing the applied magnetic field [50]. The repeated processes of creating and breaking these chains are the main source of the forces that resist fluid motion. The larger magnetic field, the stronger resistive forces against fluid flow. MRFs are very important because of their important characteristics; very fast response of changing its state from liquid to solid, quick reversibility and wide range of working conditions [60]. MRF only needs $1 \mathrm{~ms}$ to respond [37]. The presence of the resistive forces because of off/on magnetic field is the main source of the frictional torque in MRF brake. In order to get through how shear train and shear stress build through MRF, fluid models of operation are presented in the next section.

\subsubsection{MR Fluid Modes of Operation}

Generally, there are several potential applications of MRF (smart fluid); energy dissipation, brake and clutch, machine, casting and manufacturing design. Each of these applications considers one or more of fluid working conditions called working fluid modes. Magnetorheological fluid as a smart fluid can be deformed in three different modes, valve, shear, and squeeze mode [81, 82, and 83].

\subsubsection{Valve Mode}

Figure 3-5 shows working condition of MRF flow in valve mode. MRF flows between two parallel fixed plates. In order to control the fluid flow, a magnetic field is normally applied to flow direction producing a chain like structure because of dipole-

dipole interaction force between MRF particles. That means fluid flow can be controlled 
by applying a magnetic field which alters fluid viscosity. Lot of studies have discussed fluid behavior and studied fluid velocity profile and pressure drop $[84,85,86,87$, and 88]. They concluded that velocity profile and pressure drop depend on magnetic field intensity, MRF viscosity, MRF thickness, pre-yield and post-yield of fluid region, effective channel length, and inlet fluid velocity. Electromagnetic valves, dampers, and actuators are good devices that use MRF in valve mode.

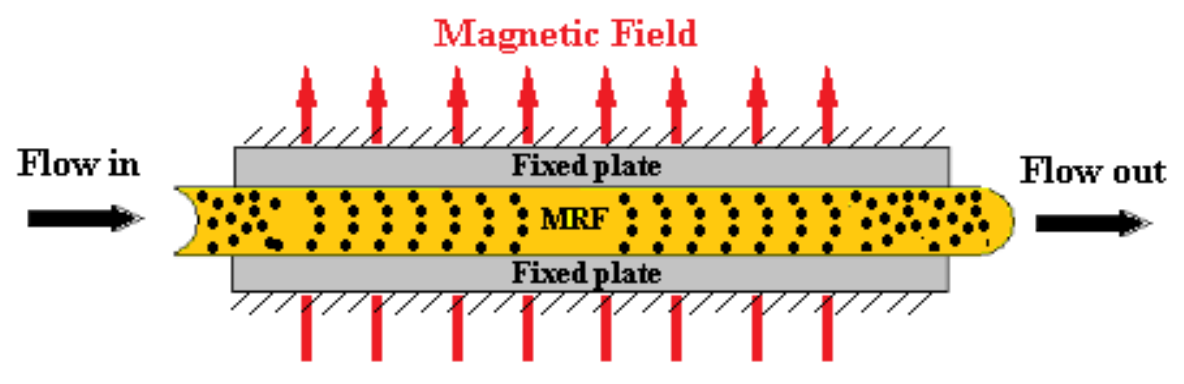

Figure 3-5. MRF in valve mode

\subsubsection{Shear Mode}

Basically, MR fluid works between two frictional surfaces in a presence of magnetic field called MRF shear mode. One of frictional surfaced could be fixed or moved relatively to the other surface. Figure 3-6 shows MRF in shear mode between two relatively parallel surfaces. In order to get higher shear stress, a magnetic field should be applied perpendicular to the direction of motion of the frictional surfaces. Shear stress is proportionally increased with increasing magnetic field. MRF brake and clutches have basically developed using MRF shear mode condition due to the fast and quick response and perfect integration between mechanical and electromagnetic parts with a high level of controllability. In addition, MRF in shear modes is used for polishing purpose by altering fluid viscosity and using special and different particles size to refine frictional surfaces. 
[90]. Bingham plastic and Bulkely models are successful models to describe shear stressstrain relationship [31, 39, 41, 43-48, 58, 59, 65, 54, 55]. Presence of shear stress implies producing a resistive shear force which can be integrated and magnified to create a restoring torque for brakes and clutched applications.

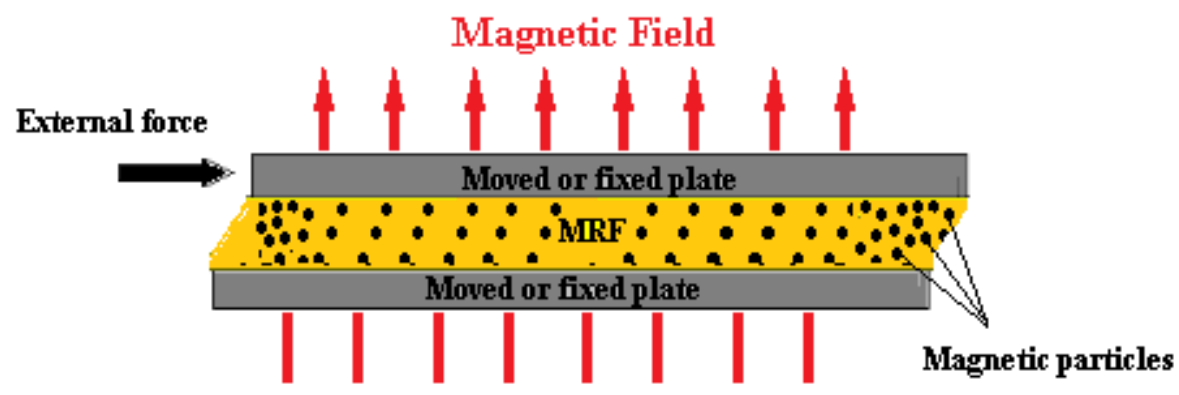

Figure 3-6. MRF in shear mode

\subsubsection{Squeeze Mode}

In this mode, MRF between two surfaces is squeezing by applying an external force normal to the surfaces. Force direction can be applied in tension or compression depending on MRF device application. Figure 3-7 shows MRF in squeeze mode. Most studies about yield stress in squeeze mode verified that yield shear stress in squeeze mode is much greater than that corresponding of shear modes [91]. Dampers are good application of MRF working in squeeze modes. MRF dampers are very quick in response and easy to control. Vibration industries are a typical device of MRF in squeeze mode especially at higher frequency transmitted wave such as high quality damper [92] and high speed engines [93]. Several researches have created to study MRF magnetic properties of MRF in squeeze mode. Some of them have proved that Bingham plastic model cannot be used to design MRF behavior in squeeze mode. The most important 
models of squeeze mode have created $[94,95]$ a bi-viscous model of MRF to study MRF yield stress with and without applied magnetic field.

MRF compression Fig. 3-7 can be achieved when the applied force normally to the surfaces to make them closer by pushing each other. MRF expands to both sides of plates. The applied normal force increases nonlinearly with magnetic field and fluid gab thickness [96]. Compression mode has studied both in static and dynamic cases [97]. Dynamic compression strain, compression time step size, and particle size are studied as well [98].

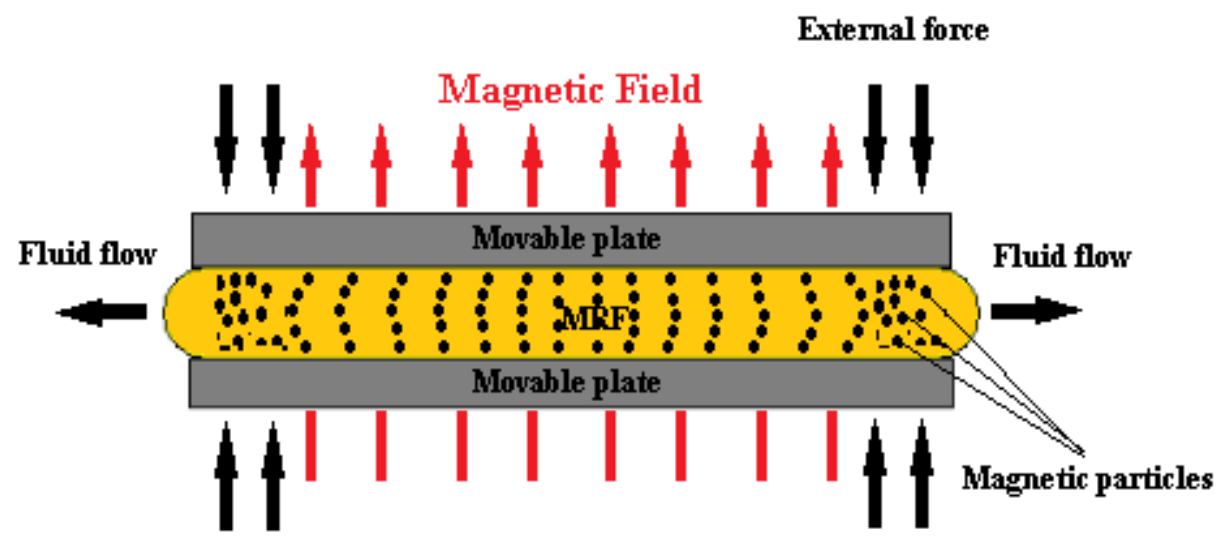

Figure 3-7. MRF in compression mode

For the tension, same procedure for compression, but the two plates move in the opposite direction (plates pulled from each other). However, for same magnetic field, fluid characteristic and yield stress in tension is lower than that corresponding in compression. Several studies have achieved in order to study MRF behavior at different conditions [99, 100]. In this research, shear mode is the working mode of MRF for brake application. The analysis and mathematical modeling is introduced in the next section. 


\subsubsection{Analysis and MRF Brake Description}

A schematic diagram of a typical magnetorheological fluid brake is shown in

Fig.s 3-8 and 3-9.
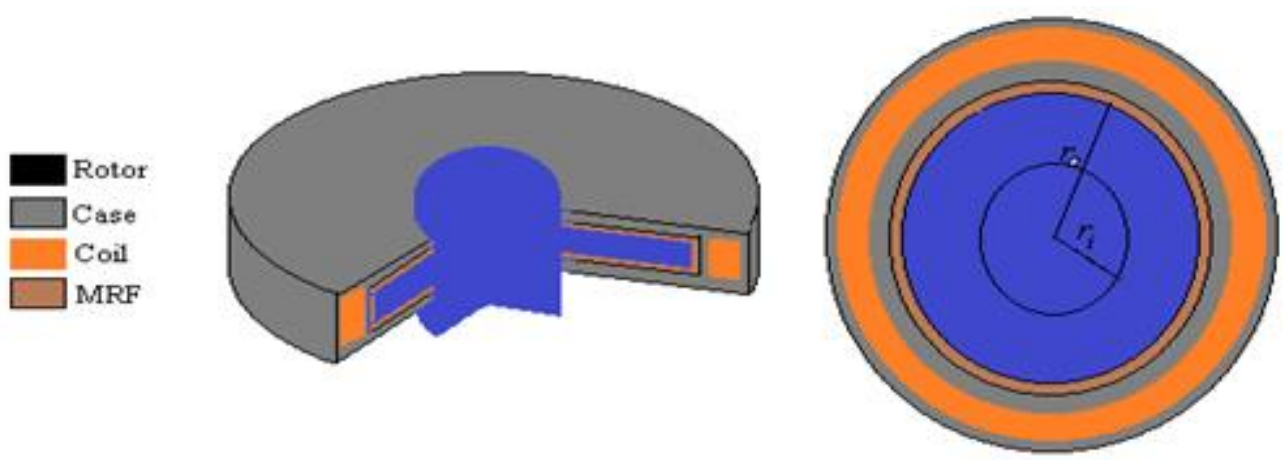

Figure 3-8. A schematic diagram of simple MRF brake

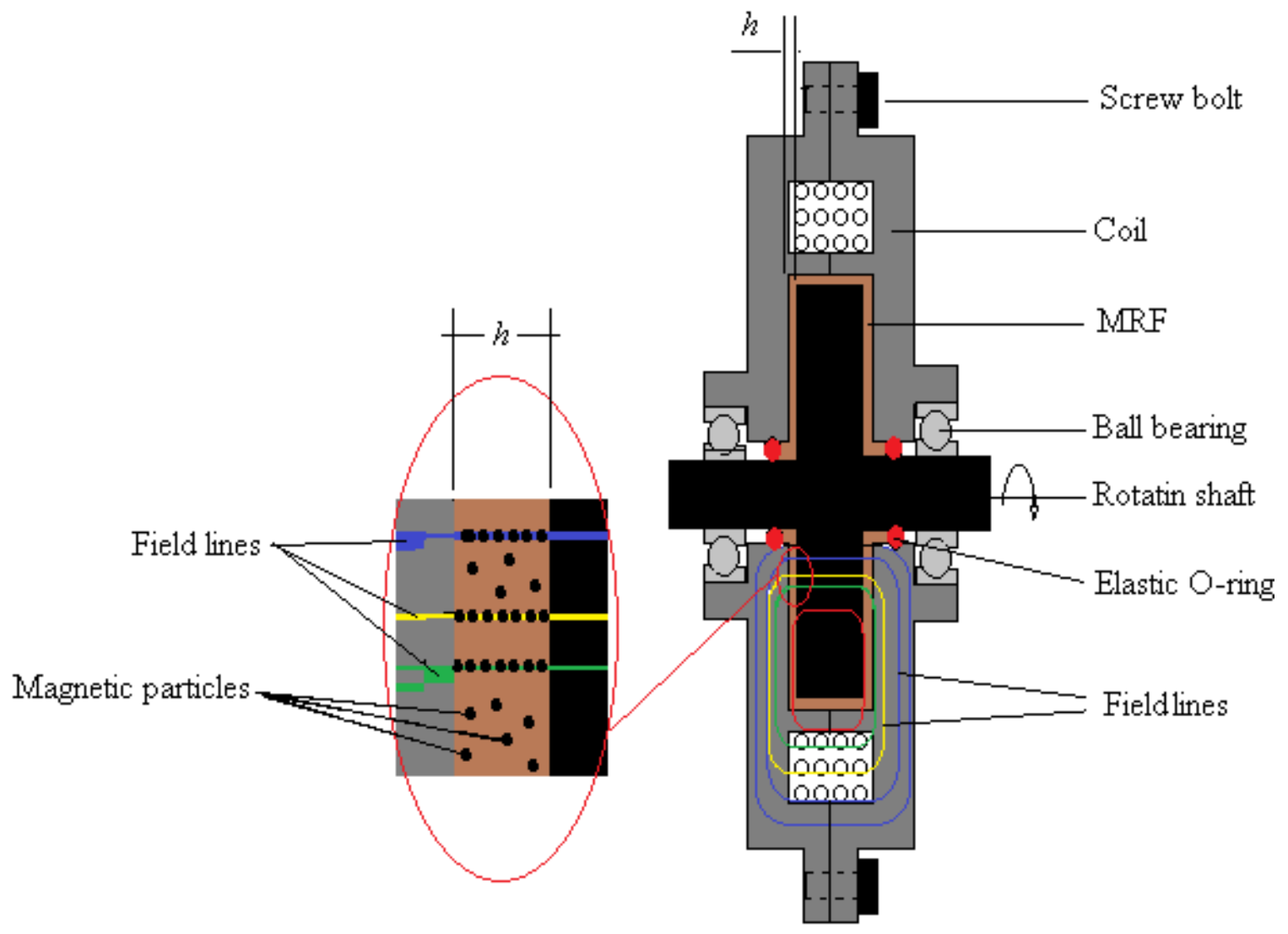

Figure 3-9. A schematic diagram of a typical magnetorheological fluid brake 
MRF brake as shown consists of several parts, screw to fasten the whole assemply of brake cases, rotaing shaft that carry a rotating disc, ball bearings in order to suupport the rotating shaft, MRF, elctrcial coil in order to create the required magnetic filed, and an elastic O-ring to prevent fluid leakage. The magnetorhelogical fluid brake is compact, simple in the design, and control. MRF brake has more advantages than conventional drum brake types. For example, on auxiliary requirements for brake activation and no brake pedal is needed. The braking process can be activated using small power supply and small controller to control an input current to a magnetic coil. The unique property of magnetorheological fluid (MRF) by altering its viscosity from liquid to partially or fully solid is the key of magnetorheological fluid brake.

In the presence of the magnetic field (due to current flow through the electrical coil), the apparent viscosity of the fluid increases because the nanoparticles change their arrangement to create like chain structure in the direction of the applied magnetic field lines. The like chains structures (like fibrils) start to prevent the fluid from flow and creating resistive forces try to resist the relative motions between brake discs.

\subsubsection{Principle of MRF Brake and Free Body Diagram Analysis}

\subsubsection{Principle of MRF Brake}

Studying magnetorheological fluid (MRF) mechanical and magnetic properties is the most important step that should be considered before MRF device design, as mentioned earlier, MRF is sensitive to a magnetic field and input current to a magnetic coil (shown at the end of this chapter). Different models are used to predict MRF behavior because of fluid viscosity and magnetic field depending on MRF assumption. 
In the normal working conditions, the disc is immersed and rotating at a specific speed. Due to the viscocity of the fluid arround the rotated disc, a retarded force appears and a retarded torque is built up as well. That torque is called a viscous torque which increase with shaft speed. However, when a current passes through an electromagnetic coil, magneitc field lines pass normally to the MRF as shown in Fig. 3-9 (colored lines). The magnetic particles start very quickly to make like chain structure throughout the magnetic lines. The new arrangement of the magnetic particles increases MRF viscosity and alters its state from fluid to fully solid. Chain like structure is very strong and its stiffness increases with increasing magnetic field and coil input current.

More specifically, the developed frictional torques in MRF brake are resulted from off-field carrier fluid viscosity, $T_{\mu}$ and from the applied magnetic field, $T_{H}$. Then, the total output, $T$, by MRF brake is given as,

$$
T=T_{\mu}+T_{H}
$$

In the presence of the fluid shear stress throughout MRF fluid, a differential force element $d f$ acting on a rotary disc differential elemental area $d A$ is exerted and can be easily given by,

$$
d f=\tau d A
$$

The resulted torque is directly affected by the differential force element and its radius (effective radius) as shown,

$$
d T=\tau d A r
$$

where $\tau$ is the fluid shear stress and $r$ is the effective radius of the corresponding elemental force as shown in Fig. 3-10. It can be easily noted that, differential element 
area $d A$ can be written as $r d r d \theta$ in terms of polar coordinates using Jacobian coordinate transformation. Based on that, the elemental torque can be given as,

$$
d T=\tau r^{2} d r d \theta .
$$

The total torque acted on the disc can be obtained using elemental torques summation over rotary disc domain as,

$$
T=\int_{0}^{2 \pi} \int_{r_{i}}^{r_{i}} \tau r^{2} d r d \theta .
$$

where $r_{o}$ and $r_{i}$ are the outer and inner radius of rotary disc as shown in Figure 3-10.

It is important to mention that torque described by Eq. (3.20) is just for one face (one effective frictional surface) of the rotary disc. So, for one disc with two effective surfaces the equation is just multiplied by 2 .

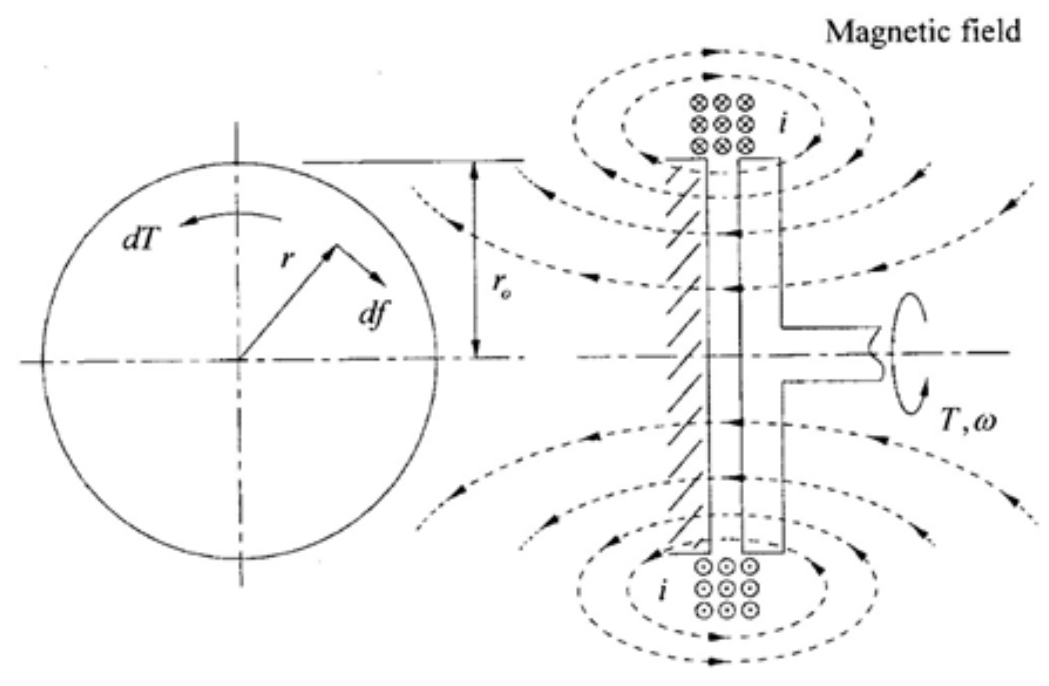

Figure 3-10. A schematic showing the geometry of MR fluid brakes and rotary disc differential elemental area

For more convenient, torque equation can be written as, 


$$
T=N \int_{0}^{2 \pi} \int_{r_{i}}^{r_{o}} \tau r^{2} d r d \theta
$$

where $N_{s}$ is the number of effective surfaces of friction. One can assume the problem to be axisymmetric and make torque equation much easier as shown,

$$
T=2 \pi N_{s} \int_{r_{i}}^{r_{o}} \tau r^{2} d r
$$

That means the braking torque is independent on an orientation angle.

\subsubsection{Fluid Shear Stress}

Shear stress is mainly contributed by MRF fluid viscosity filled through the gap between fixed and rotary discs $\tau_{P}$ and due to the applied magnetic field $\tau_{H}$ where the first and second terms are shear stress due to plastic viscosity and magnetic field, respectively. Bingham plastic model, which assume a linear viscosity with strain rate as in Fig. 3-11, has widely used to describe MRF behavior [31, 39, 41, 43-48, 58, 59, 65, 54, 55].

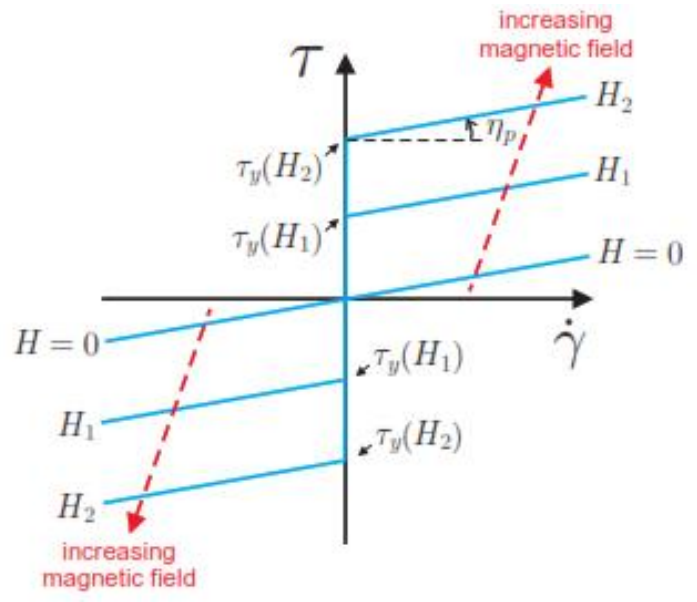

Figure 3-11. Bingham plastic model of an MRF shear stress verses shear rate [101] 
The combination effect of both viscous and magnetic fluid shear stresses can be given as,

$$
\tau=\tau_{H}+\tau_{P}
$$

where $\tau_{H}$ and $\tau_{P}$ are shear stresses due to magnetic field and MRF viscosity,

respectively. The second term in Eq. (3.23) is a function of fluid viscosity and fluid shear strain due fluid dynamic viscosity and shear strain rate.

\subsubsection{Fluid Shear Strain}

Bingham plastic and Herschel-Bulkely models consider how shear stress changes with yield strain and shear strain depending on fluid behavior assumption, Newtonian or non-Newtonian fluid. Newtonian fluid can be defined as the fluid that shear stress is linearly proportional to shear strain. In other words, shear stress and shear strain have same relation on shear stress-strain line. Hence, viscosity of a Newtonian fluid still constant with time and shear rate as well. In contrast, when a fluid viscosity changes with time and shear rate, it is called non-Newtonian fluid. Bingham plastic and dilatants fluid are good examples about non-Newtonian fluid [102].

A non-Newtonian fluid starts to flow when shear stress become greater than a critical value. Non-Newtonian fluid can be classified into two groups, shear thinning and shear thickening fluids depending on fluid dynamic viscosity behavior. Dynamic shear viscosity decreases with a strain rate for shear thinning fluid. However it increases with shear strain rate for shear thickening fluid as shown in Fig. 3-12. [92]. In the normal conditions (absence of magnetic field), MRF can be considered as a Newtonian fluid. Studying fluid characteristics and obtaining a relationship between shear stress and shear 
strain is a basic step for a mathematical modeling. Figure 3-13 shows an infinitesimal fluid element subjected to shear forces on upper and lower faces.

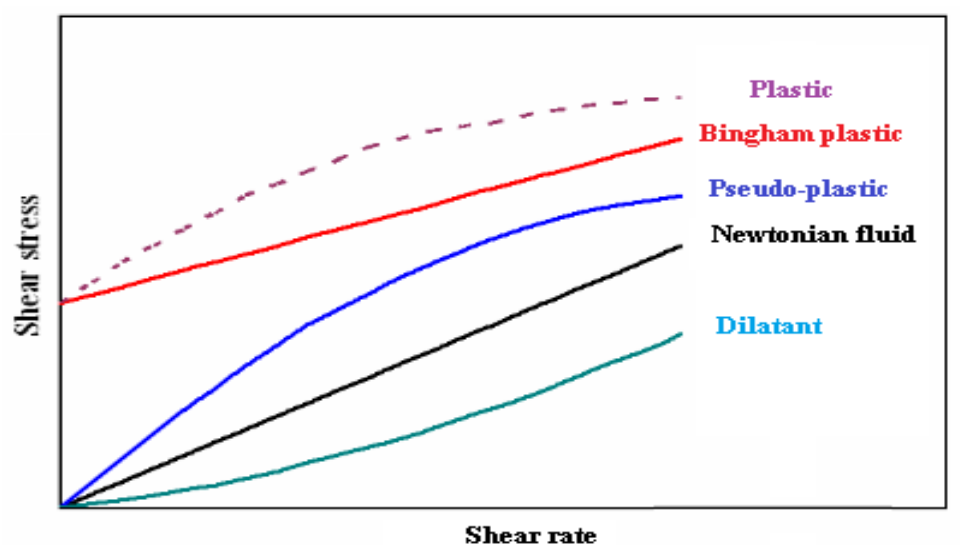

Figure 3-12. Different behaviors of fluid response to shear rate [102]

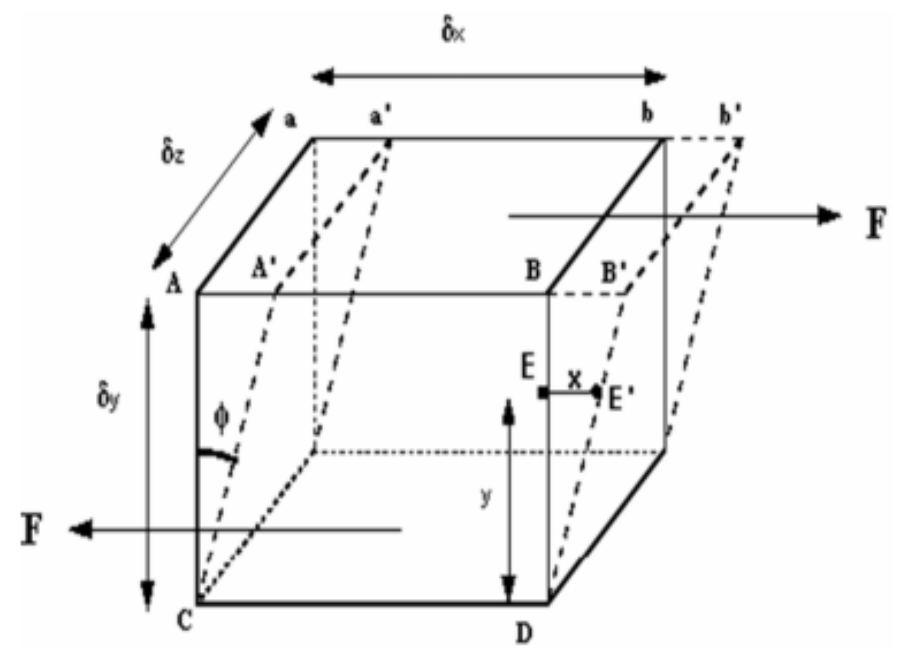

Figure 3-13. Differential fluid element subjected to shear forces [103]

Elemental shear stress can be then calculated by $d f=\tau d A$, where $d A=\delta_{x} \delta_{y}$.

Shear stress deforms a fluid element producing a shear strain which can be represented by shear strain $\left(\gamma_{s}\right)$ at any point E' as,

$$
\gamma_{s}=\frac{x}{y}
$$


Shear strain rate $\dot{\gamma}_{s}$ then can be given in terms of point velocity,

$$
\dot{\gamma}_{s}=\frac{V_{x}}{y}
$$

where $V_{x}$ is a point velocity in x-direction. For each element, shear stress is proportional to shear strain rate so that,

$$
\tau_{P} \propto \dot{\gamma} \Rightarrow \tau_{P}=\mu_{m M R F} \dot{\gamma}
$$

where $\mu_{m M R F}$ is MRF viscosity.

\subsubsection{Shear Strain Rate of MRF Brake}

Since the concern is about studying sensitivity analysis and obtain important and significant parameters that affect the preliminary MRF brake design, several assumptions can be adopted to make the processes more convenient. They are:

1- The fluid flow through fluid gap is fully developed and laminar flow.

2- Newtonian fluid behavior in the normal conditions.

3- No body force due to the centrifugal effect.

4- Fluid gap (thickness) is very small as compared to disc radius.

With the above assumptions, shear strain rate can be represented by the velocity gradient or velocity changes along fluid gap for a typical MRF brake design. So, Eq. (3.25) can be

updated to obtain $\dot{\gamma}_{s}=\frac{V_{r}-V_{s}}{d z}$ where the numerator is the difference between rotor and stator speeds and the denominator is the fluid gap element. $V_{s}=0$ for a typical MRF brake application and $V_{r}$ can be described by rotor rotational speed $(\omega)$ and effective disc radius $(r)$.The radial velocity can be given by $V_{r}=r \omega$. It can be easily considered that 
fluid velocity is linearly distributed and then shear strain rate given by Eq. (3.25) can be given by,

$$
\dot{\gamma}=\frac{r \omega}{h}
$$

where $h$ is the fluid thickness.

On the other side, the shear stress due to the magnetic field $\tau_{H}$ can be defined by constants and magnetic field intensity as shown,

$$
\tau_{H}=k H^{\beta}
$$

where $k$ and $\beta$ are constants and can be defined using best curve fitting from the relationship between yield stress and magnetic field intensity $(H)$ where $\mathrm{H}$ and $\mathrm{k}$ are measured by $\mathrm{A} / \mathrm{m}$ and $\mathrm{Pa} . \mathrm{m} / \mathrm{A}$, respectively. This relation is commonly given by a company that provides MRF. Using Eq.s (3.25)-(3.28) and Eq.s (3.22) and (3.23), the total torque can be described by,

$$
T=2 \pi N \int_{r_{i}}^{r_{o}}\left(k H^{\beta} \operatorname{sign}(\dot{\gamma})+\mu_{m M R F} \frac{r \omega}{h}\right) r^{2} d r
$$

More specifically, the total brake can be divided into two parts. The first one due to fluid viscosity and the second is due to magnetic field as shown,

$$
\begin{aligned}
& T_{P}=2 \pi N \int_{r_{i}}^{r_{o}} \mu_{m M R F} \frac{\omega}{h} r^{3} d r . \\
& \text { and } \\
& T_{H}=2 \pi N \int_{r_{i}}^{r_{o}} k H^{\beta} \operatorname{sign}(\dot{\gamma}) r^{2} d r .
\end{aligned}
$$

The magnetic field $H$ is not constant in general, but it varies along the disc radius. The magnetic field is largely depending on the electrical circuit design and coil 
location. Actually, there is no exact solution to describe that relation; however, it can be calculated using numerical analysis like finite element method. For some cases, the magnetic field can be assumed constant and then Eq. (3.30) can be easily integrated as follows,

$$
\begin{aligned}
& T_{P}=\frac{\pi N_{s} \omega \mu_{m M R F}}{2 h}\left(r_{o}^{4}-r_{i}^{4}\right) \\
& T_{H}=\frac{2 \pi N_{s} k H^{\beta}}{3}\left(r_{o}^{3}-r_{i}^{3}\right)
\end{aligned}
$$

One can easily note that there are two groups of parameters that affect the total torque; the first is relevant to the geometrical design like number of contact surfaces $N_{s}$, inner and outer disc radii $r_{i}$ and $r_{o}$, and fluid gap. While the second group is relevant to fluid and magnetic properties $k, \beta, \mu_{m M R F}$ and $H$. It worth to note that $k$ and $\beta$ can be defined depending on what type of MRF fluid used and experiments (shear stressmagnetic field relationship) that have been done by manufacturers. Some of literature assumes a linear relationship with current [33]. General introduction about the magnetic circuit of magnetorheological fluid brake is given in the next section.

\subsubsection{Magnetic Circuit Design}

\subsubsection{General}

In order to understand the way of producing the electromagnetic field, a general concept of magnetism is presented. Electromagnetism is the relationship between electricity and magnetism that describes how a magnetic field produces an electrical power and how electrical power produces a magnetic field. In this work, the concentration will be on the electricity induced magnetic field. The conventional way of 
producing a magnetic field can be done using an electromagnetic coil as shown in Fig. 314. When the electrical current passes through coil windings as shown in Fig. 3-14b, a magnetic field is developed and magnetic lines appear from the center of the coil to the coil sides as well. The developed magnetic flux density can be given by,

$$
B=\frac{\mu_{m} N i}{\sqrt{4 r^{2}+l^{2}}}
$$

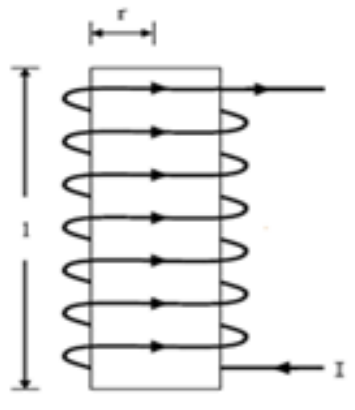

(a)

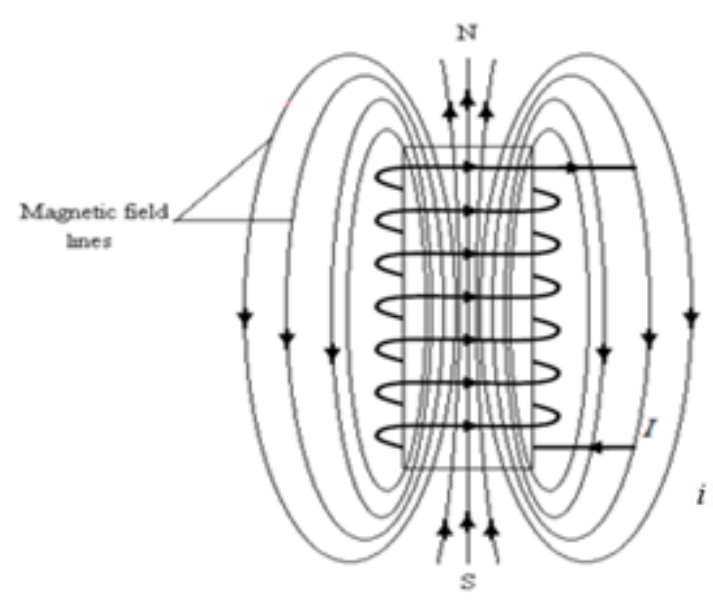

(b)

Figure 3.14. Wounded coil with input current and induced magnetic field lines

where $B$ is the magnetic flux density measured by Tesla or Weber $\left(\mathrm{Web} / \mathrm{m}^{2},\right) N$ is the number of coil windings, $i$ is the input current to the electromagnetic coil, $\mu_{m}$ is the magnetic permeability of coil core, $r$ is the radius of windings, and $l$ is the coil length as shown in Figure 3-14a. In case of coil length is greater than winding radius; Eq. (3.32) can be given as,

$$
B=\frac{\mu_{m} N i}{2 l}
$$

Another important factor is called magnetic field intensity which is largely depends on 
coil length and current passing through windings. Ampere suggest the relationship between $H, l$, and $i$ as shown,

$$
\int H . d l=i
$$

where the electrical current $i$ is the line integral of the magnetic field intensity along coil path. Magnetic intensity is a measurement of current per unit length of the coil and can be given by $\mathrm{A} / \mathrm{m}$. In terms of magnetomotive force which is depends directly on a current and length, Eq. (3.34) can be given by,

$$
\int f H . d l=N i=F(m m f)
$$

where $\mathrm{mmf}$ refers to magnetomotive force. Equation (3.35) called the steady-state Maxwell- Ampere's Law [104]. For more convenient, Eq. (3.35) can be simplified into,

$$
H=\frac{N i}{l}
$$

Magnetic flux density can be given as a function of coil core permeability and magnetic field. In case of air gab instead of metal coil core, magnetic density can be given by [105]

$$
B=\mu_{o} H
$$

where $\mu_{o}$ is the magnetic permeability of air gap $\left(4 \pi * 10^{-7} \mathrm{~T} . \mathrm{m} / \mathrm{A}\right)$. If the core of magnetic coil is made of some material of permeability $\mu_{m}$, Eq. (3.37) can be modified to be $[105,106]$

$$
B=\mu_{m} H
$$

Some references use relative permeability $\mu_{r}$ instead if air and material permeability so that $\mu_{r}=\mu_{m} / \mu_{o}$ and then, 


$$
B=\mu_{r} \mu_{o} H
$$

In addition, magnetic field Eq.s (3.35) and (3.36) can be re-written for sections of magnetic circuit as,

$$
\int\left[H . d l=\sum_{i} H_{i} l_{i}=N i=F(m m f)\right.
$$

where index $i$ refers to section index. Since MRF brake consists of several sections, then Eq.(3.40) is given by,

$$
\int H . d l=N i=H_{M R F} l_{M R F}+\sum_{j} H_{j} l_{j}
$$

where $j$ refers to the other sections of MRF brake material sections as shown in Fig. 3.15.

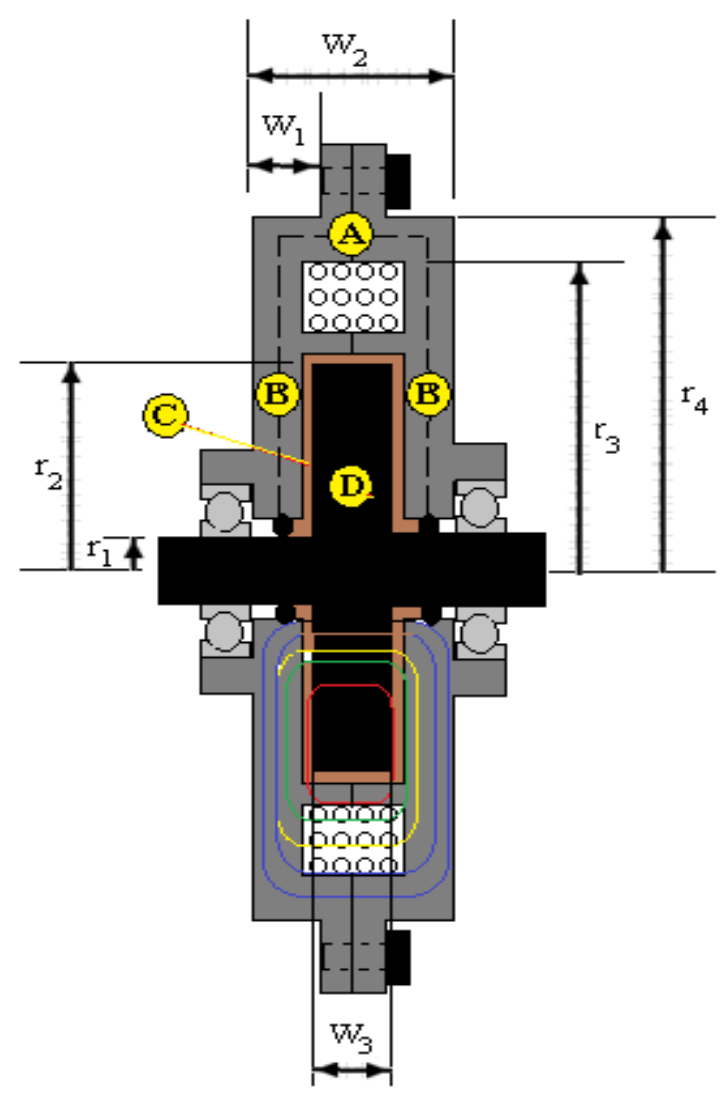

Figure 3-15. Magnetic circuit of sections of MRF brake 


\subsubsection{Candidate Material used for MRF Brake}

Magnetorheological fluid (MRF) is manufactured using metallic materials and their mechanical and thermal properties can be affect MRF brake performance. Therefore, it is important to study these properties. Beside their mechanical and thermal properties, their magnetic properties are very important as well. For example, magnetic permeability is an important factor that greatly affects the magnetic intensity of a magnetic circuit. To increase the magnetic performance and magnetic properties at the MRF section, magnetic field intensity through fluid region need to be increased. To do so, magnetic permeability for brake metallic material needs to be increased especially those regions close to MRF and faces the magnetic coil. Magnetic permeability is explained in the next section. Another important factor should be taken into the consideration during selection of MRF brake materials is the magnetic saturation. A material of higher magnetic saturation is preferable because it offers higher magnetic permeability. Ferrous material of high magnetic permeability must be used in MRF brake design in order to reduce the magnetic field intensity through metallic brake material and increases its value at MRF, thereby, decreases the required input current.

\subsubsection{Magnetic Field Intensity and Brake Dimensions}

As seen previously, that braking torque due to the magnetic field is the main source of MRF braking torque, it is critical to increase the magnetic field at MRF section to increase magnetic force of the magnetic field lines and get stronger chain like structure. Some magnetic properties and their definition are given in the next section. 


\subsection{Magnetic Reluctance}

Magnetic reluctance can be considered as one of the most complicated parts through design magnetic circuit. However, there are some successful methods used to put a good mathematical modeling. A magnetic flux passing through a surface area $A_{s}$, can be given as a function to magnetic field density according to Kirchhoff's law as shown [107],

$$
\phi=\int B . d A
$$

So that the total magnetic flux is the summation of all normal components of flux density of the surface as shown in Fig. 3-16 where $A_{s}$ an elemental area normal to the surface.

For uniform flux density, the magnetic flux is given by,

$$
\phi=B A_{s}
$$

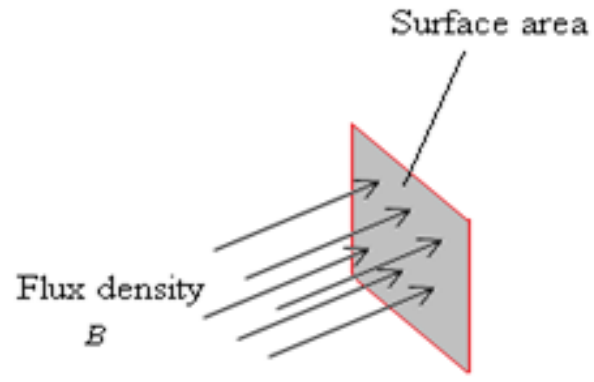

Figure 3-16. Basic quantities of the magnetic circuit

The easiest method considers that magnetic flux $\phi$ (Weber) can be given as,

$$
\phi_{i}=B_{i} A_{i}
$$

Similar analogy of electrical energy (to obtain the relationship between current, voltage, and electrical resistance) can be used to obtain the relationship between (magnetomotive 
force, magnetic flux, and magnetic reluctance so that,

$$
\mathfrak{R}_{i}=\frac{F(m m f)}{\phi_{i}}
$$

where $\mathfrak{R}_{i}$ is a magnetic reluctance for part $i$. Using Eq. (3.40) and (3.45), a magnetic reluctance can be given by,

$$
\mathfrak{R}_{i}=\frac{1}{\mu_{m i}} \frac{l_{i}}{A_{s i}}
$$

where $\mathfrak{R}_{i}$ is the magnetic reluctance [104].

It worth to note that magnetic reluctance depends on geometrical design of the path length $l_{i}$ and cross-sectional area $A_{s i}$. Therefore, it is good to find an equivalent magnetic reluctance for the magnetic circuit instead of treating them locally. One can think about reluctance as a picture similar to electrical resistance. So, they can be added in parallel or series. Parallel addition can be governed by,

$$
\frac{1}{\mathfrak{R}_{\text {eqv }}}=\sum_{i} \frac{1}{\mathfrak{R}_{i}}
$$

where $\mathfrak{R}_{\text {eqv }}$ and $\mathfrak{R}_{i}$ are equivalent and section $i$ reluctance, respectively. For series addition,

$$
\mathfrak{R}_{\text {eqv }}=\sum_{i} \mathfrak{R}_{i}
$$

Some cases at which the magnetic flux is not parallel due to magnetic flux variations is presented below. It is a little different. The general idea is to pretend that the volume that belonged to the magnetic flux can be represented by collection of separate flux tubes as shown in Fig. 3-17 making a closed wall parallel to the flux paths. An elemental volume defined by $d A$ and $d l$ and an associated reluctance can be given by [108], 


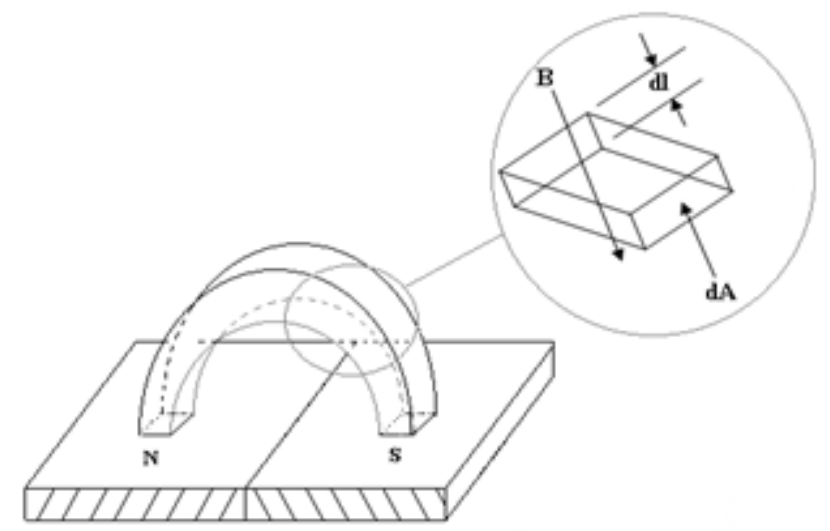

Figure 3-17. Reluctance element of non-uniform flux path

$$
\mathfrak{R}=\frac{1}{\mu_{m}} \int \frac{d l}{A}
$$

where $l$ and $A$ are magnetic flux path length and A is the cross-sectional area. The crosssectional area in Eq. (3.49) in the radial direction is $A=r \vartheta l$ where $\vartheta$ is the arc angle measured in degrees. Using the cross-sectional area with Eq. (3.49), the magnetic reluctance is given by,

$$
\mathfrak{R}=\frac{1}{\mu_{m}} \int_{i}^{j} \frac{d r}{r \vartheta l}
$$

Since the angle $\vartheta=2 \pi$ and length are constants, then

$$
\mathfrak{R}=\frac{1}{2 \pi \mu_{m} l} \ln \left(\frac{r_{j}}{r_{i}}\right)
$$

\subsection{Magnetic Reluctance of MRF Brake}

Now, it is easy to use the general equations of magnetic permeability to calculate the individual reluctance of magnetic parts for MRF brake as shown in Figure 3-15. The magnetic reluctance can be given by, 


$$
\begin{aligned}
& \mathfrak{R}_{A}=\frac{W_{2}}{\pi \mu_{m A}\left(r_{4}^{2}-r_{3}^{2}\right)} . \\
& \mathfrak{R}_{B}=\frac{1}{2 \pi \mu_{m B} W_{1}} \ln \left(\frac{r_{4}}{r_{3}}\right) . \\
& \mathfrak{R}_{C}=\frac{h}{\pi \mu_{m M R F}\left(r_{2}^{2}-r_{1}^{2}\right)} . \\
& \mathfrak{R}_{D}=\frac{W_{3}}{\pi \mu_{m A}\left(r_{2}^{2}-r_{1}^{2}\right)} .
\end{aligned}
$$

where $W_{1}, W_{2}$ and $W_{3}$ are sections thicknesses, $r_{1}, r_{2}, r_{3}$, and $r_{4}$ are sections radii as

shown in Fig. 3-15. The equivalent reluctance of the magnetic circuit can be given by,

$$
\mathfrak{R}_{\text {eqv }}=\mathfrak{R}_{A}+2 \mathfrak{R}_{B}+2 \mathfrak{R}_{C}+\mathfrak{R}_{D}
$$

The equivalent magnetic flux is a function of equivalent magnetic reluctance as,

$$
\phi_{\text {eqv }}=\frac{N i}{\mathfrak{R}_{\text {eqv }}}
$$

where $\mathfrak{R}_{\text {eqv }}$ and $\phi_{\text {eqv }}$ are equivalent reluctance and magnetic flux, respectively. In order to increase an equivalent magnetic flux, one can increase the numerator (increase input current or number of coil turns) or decrease the denominator (equivalent reluctance). However, increasing the input current is not the good way for that because of magnetic saturation problem [37, 133-136].

\subsubsection{Brake Efficiency}

One of the most important criteria that used to evaluate and compare function and performance of a magnetorheological fluid brake is brake efficiency. Brake efficiency can be easily defined by a ratio between an output to an input. For a magnetorheological fluid brake the mechanical power is the output and the electrical power required for 
activation is the input. Brake efficiency is given by,

$$
E_{b}=\frac{\left(T_{P}+T_{H}\right) w}{P_{e}}
$$

where $E_{b}$ is the brake efficiency and $P_{e}$ is the electrical power (Watt).

Generally speaking, magnetorheological fluid brake efficiency can be increased by either increasing the braking torques (plastic and magnetic) or by decreasing the electrical power required for activation. Increasing the braking torques can be done by getting a best design of the brake taking the advantage of sensitivity studies and Taguchi optimization approach. The input electrical power can be given as follows [37],

$$
P_{e}=i^{2} R
$$

where $R$ is the wire resistance (Ohm). The wire resistance can be given by [37],

$$
R=2 \pi r_{m} k_{w} N / S_{w}
$$

where $r_{m}$ is the mean radius of the electrical coil, $k_{w}$ is the resistivity of the wire and $S_{w}$ is the wire section. For more convenient, using Eq.s (3.56) and (3.57) together, the electrical power can be given as follows [37],

$$
P_{e}=2 \pi r_{m} k_{w} v N I
$$

where the symbol $v$ is the ratio between the applied current to the surface area $\left(\mathrm{A} / \mathrm{m}^{2}\right)$. Using Eq.s (3.58) and (3.54) the electrical power is given as a function of the equivalent magnetic circuit reluctance by,

$$
P_{e}=2 \pi r_{m} k_{w} v \phi_{e q v} \mathfrak{R}_{e q v}
$$

One way to decrease the electric power can be achieved by decreasing the equivalent magnetic reluctance $\mathfrak{R}_{e q v}$. As seen previously from Eq. (3.52) that magnetic 
reluctances for the magnetorheological fluid brake are functions of brake radii. It is important to understand how brake radii affect magnetic reluctances and get the best way to make it as small as possible. However, magnetorheological fluid brake torques (plastic and magnetic) are functions of brake radii as well. It is worth to study a sensitivity analysis for MRF brake (torques and reluctance) and explain how both of them are related each other and make a tradeoff between them to get the best design.

\subsubsection{Sensitivity Analysis for MRF Brake and Magnetic Circuit}

In order to increase braking torque, one can change the design parameters in some ways and maybe by making a tradeoff between them. For example, torque can be increased by either increasing number of frictional surfaces or by using a high viscosity fluid and smaller fluid gap. However, either ways may be limited by geometrical space limitations (because of increasing number of frictional surface, as an example) or may lead to increase off-field torque which unfavorable (because of using an MRF of high viscosity). In addition, using fluid with higher permeability for magnetic field and good electrical circuit design may increase braking torque. Based on that, it is important to understand how design parameters affect braking torque and obtain which of them have a critical impact on the brake performance. Sensitivity analysis is very important to know that.

\subsubsection{Nondimensional Form of MRF braking torque}

To conduct a sensitivity analysis that follows, the torque shown in Eq. (3.31) will be nondimensionalized using the following definitions: 


$$
\begin{gathered}
r_{i}=\hat{r}_{i} r_{o}, h=\hat{h} h_{o}, \quad w=\hat{w} w_{o}, \quad \mu_{m M R F}=\hat{\mu}_{m M R F} \mu_{o}, \tau_{H}=\hat{\tau}_{H} \cdot \tau_{H_{o}}, k=\hat{k} k_{o}, H^{\beta}=\hat{H}^{\hat{\beta}} H_{o}^{\beta_{o}} \\
T_{p}=\frac{\pi}{2} \frac{N w_{o} \mu_{o}}{h_{o}} r_{o}^{4} \hat{T}_{P}, T_{H}=\frac{2 \pi}{3} N k_{o} H_{o}^{\beta_{o}} r_{o}^{3} \cdot \hat{T}_{H}
\end{gathered}
$$

In this equation, the symbols with carets are nondimensional. Substituting Eq. (3.60) into

Eq. (3.31) produces the following expressions for the nondimensional braking torque:

$$
\hat{T}_{P}=\left(1-\hat{r}_{i}^{4}\right) \cdot \frac{\hat{w} \hat{\mu}_{m M R F}}{\hat{h}}
$$

Similarly,

$$
\hat{T}_{H}=\hat{k} \hat{H}^{\hat{\beta}}\left(1-\hat{r}_{i}^{3}\right)
$$

From this equation, it may be seen that the braking viscous torque $\left(T_{p}\right)$ depends upon

four nondimensional design parameters: $\hat{w}, \hat{h}, \hat{\mu}$, and $\hat{r}_{i}$, while the magnetic torque

depends upon $\hat{k}, \hat{H}, \hat{\beta}$, and $\hat{r}_{i}^{3}$.In other words, MRF brake torque depends on seven nondimensional design parameters only. In the following section, Taylor series will be used to conduct sensitivity analysis for the purposes of identifying the most sensitive parameters that can be adjusted in order to alter the physical characteristics of the brake.

\subsubsection{Sensitivity Studies of MRF braking torque}

The sensitivity analysis for the MRF braking torque will be conducted using a first order Taylor series expansion for the torque equation presented in Eq.s (3.61) and (3.62). This Taylor series expression is;

$$
\hat{T}_{p}=\hat{T}_{p_{o}}+\left.\frac{\partial \hat{T}_{p}}{\partial \hat{r}_{i}}\right|_{o}\left(\hat{r}_{i}-\hat{r}_{i o}\right)+\left.\frac{\partial \hat{T}_{p}}{\partial \hat{w}}\right|_{o}\left(\hat{w}-\hat{w}_{o}\right)+\left.\frac{\partial \hat{T}_{p}}{\partial \hat{\mu}}\right|_{o}\left(\hat{\mu}-\hat{\mu}_{o}\right)+\left.\frac{\partial \hat{T}_{p}}{\partial \hat{h}}\right|_{o}\left(\hat{h}-\hat{h}_{0}\right)
$$

This result can be rearranged and written as 


$$
\left(\frac{\hat{T}_{p}}{\hat{T}_{p_{o}}}-1\right)=\underbrace{\left.\frac{\partial \hat{T}_{p}}{\partial \hat{r}_{i}} \frac{\hat{r}_{i}}{\hat{T}_{p}}\right|_{o}}_{S_{\hat{r}}}\left(\frac{\hat{r}_{i}}{\hat{r}_{i o}}-1\right)+\underbrace{\left.\frac{\partial \hat{T}_{p}}{\partial \hat{w}} \frac{\hat{w}}{\hat{T}_{p}}\right|_{0}}_{S \hat{w}}\left(\frac{\hat{w}}{\hat{w}_{o}}-1\right)+\underbrace{\left.\frac{\partial \hat{T}_{p}}{\partial \hat{\mu}} \frac{\hat{\mu}_{m M R F}}{\hat{T}_{p}}\right|_{0}}_{S_{\hat{\mu}}}\left(\frac{\hat{\mu}_{m M R F}}{\hat{\mu}_{m M R F_{0}}}-1\right)+\underbrace{\left.\frac{\partial \hat{T}_{p}}{\partial \hat{h}} \frac{\hat{h}}{\hat{T}_{p}}\right|_{o}}_{S_{\hat{h}}}\left(\frac{\hat{h}}{\hat{h}_{o}}-1\right)
$$

where the sensitivity coefficients are given by,

$$
S_{\hat{r}_{i}}=\frac{-4 \hat{r}_{i o}^{4}}{\left(1-\hat{r}_{i o}^{4}\right)}, \quad S_{\hat{w}}=1, S_{\hat{h}_{i}}=-1, S_{\hat{\mu}_{i}}=1
$$

And for the magnetic field torque,

$$
\hat{T}_{H}=\hat{T}_{H_{o}}+\left.\frac{\partial \hat{T}_{H}}{\partial \hat{r}_{i}}\right|_{o}\left(\hat{r}_{i}-\hat{r}_{i o}\right)+\left.\frac{\partial \hat{T}_{H}}{\partial \hat{k}}\right|_{o}\left(\hat{k}-\hat{k}_{o}\right)+\left.\frac{\partial \hat{T}_{H}}{\partial \hat{\beta}}\right|_{o}\left(\hat{\beta}-\hat{\beta}_{o}\right)+\left.\frac{\partial \hat{T}_{H}}{\partial \hat{H}}\right|_{o}\left(\hat{H}-\hat{H}_{0}\right)
$$

This result can be rearranged and written as

$$
\left(\frac{\hat{T}_{H}}{\hat{T}_{H_{o}}}-1\right)=\underbrace{\left.\frac{\partial \hat{T}_{H}}{\partial \hat{r}_{i}} \frac{\hat{r}_{i}}{\hat{T}_{H}}\right|_{o}}_{S_{\hat{r}}}\left(\frac{\hat{r}_{i}}{\hat{r}_{i}}-1\right)+\underbrace{\left.\frac{\partial \hat{T}_{k}}{\partial \hat{k}} \frac{\hat{k}}{\hat{T}_{H}}\right|_{o}}_{S_{\hat{k}}}\left(\frac{\hat{k}}{\hat{k}_{o}}-1\right)+\underbrace{\left.\frac{\partial \hat{T}_{H}}{\partial \hat{\beta}} \frac{\hat{\beta}}{\hat{T}_{H}}\right|_{o}}_{S_{\hat{\beta}}}\left(\frac{\hat{\beta}}{\hat{\beta}_{o}}-1\right)+\underbrace{\left.\frac{\partial \hat{T}_{H}}{\partial \hat{H}} \frac{\hat{H}}{\hat{T}_{H}}\right|_{o}}_{S_{\hat{H}}}\left(\frac{\hat{H}}{\hat{H}_{o}}-1\right)
$$

where the sensitivity coefficients are given by

$$
S_{\hat{r} H}=\frac{-3 \hat{r}_{i o}^{3}}{\left(1-\hat{r}_{i o}^{3}\right)}, S_{\hat{k}}=1, S_{\hat{H}}=\hat{\beta}_{o}, S_{\hat{\beta}}=\hat{\beta}_{o} \log \left(\hat{H}_{o}\right)
$$

The sensitivity coefficient with the large absolute value will indicate that a plus or minus percent change in that parameter will create the largest impact on the torque capacity of the MRF brake.

\subsubsection{Nondimensional Form of MRF Brake Magnetic Circuit}

In order to conduct a sensitivity analysis that follows, the magnetic reluctance shown in Eq. (3.52) will be nondimensionalized using the following definitions: Let; 


$$
\begin{gathered}
r_{1}=\hat{r}_{1} r_{2 o}, r_{3}=\hat{r}_{3} r_{2 o}, r_{2}=\hat{r}_{2} r_{2 o}, r_{4}=\hat{r}_{4} r_{2 o}, h=\hat{h} r_{2 o}, W_{1}=\hat{W}_{1} r_{2 o}, W_{2}=\hat{W}_{2} r_{2 o}, W_{3}=\hat{W}_{3} r_{2 o}, \mu_{i m}=\hat{\mu}_{i m} \mu_{o} \\
\text { and } \\
\mathfrak{R}_{A}=\hat{\mathfrak{R}}_{A} \frac{1}{r_{2 o} \mu_{o}}, \mathfrak{R}_{B}=\hat{\mathfrak{R}}_{B} \frac{1}{\mu_{o}}, \mathfrak{R}_{C}=\hat{\mathfrak{R}}_{C} \frac{1}{\mu_{o} r_{2 o}}, \mathfrak{R}_{D}=\hat{\mathfrak{R}}_{D} \frac{1}{r_{2 o} \mu_{o}}
\end{gathered}
$$

In this equation, the symbols with carets are nondimensional. Substituting Eq. (3.69) into Eq. (3.52) produces the following expressions for the magnetic reluctances:

For the magnetic reluctance at section A:

$$
\hat{\mathfrak{R}}_{A}=\frac{\hat{W}_{2}}{\pi \hat{\mu}_{A m}\left(\hat{r}_{4}^{2}-\hat{r}_{3}^{2}\right)}
$$

For the magnetic reluctance at section $\mathrm{B}$ :

$$
\hat{\mathfrak{R}}_{B}=\frac{1}{2 \hat{\mu}_{B m} \hat{W}_{1}} \ln \left(\frac{\hat{r}_{4}}{\hat{r}_{3}}\right)
$$

For the magnetic reluctance at section $\mathrm{C}$ :

$$
\hat{\mathfrak{R}}_{C}=\frac{\hat{h}}{\pi \hat{\mu}_{C m}\left(\hat{r}_{2}^{2}-\hat{r}_{1}^{2}\right)}
$$

For the magnetic reluctance at section D:

$$
\hat{\mathfrak{R}}_{D}=\frac{\hat{W}_{3}}{\pi \hat{\mu}_{D m}\left(\hat{r}_{2}^{2}-\hat{r}_{1}^{2}\right)}
$$

From the above equations, it may be seen that the four magnetic reluctances depend upon nine nondimensional design parameters: $\hat{r}_{1}, \hat{r}_{2}, \hat{r}_{3}, \hat{r}_{4}, \hat{h}, \hat{W}_{1}, \hat{W}_{2}, \hat{W}_{3}$ and $\hat{\mu}_{i m}$. In the following section, Taylor series will be used to conduct sensitivity analysis for the purposes of identifying the most sensitive parameters that can be adjusted in order to alter the physical characteristics of the brake. 


\subsubsection{Sensitivity Studies of MRF Magnetic Circuit}

The sensitivity analysis for the MRF braking torque will be conducted using a

first order Taylor series expansion for the torque in Eq. s (3.70 to 3.73).

For the nondimensional reluctance $\hat{\mathfrak{R}}_{A}$;

$\hat{\mathfrak{R}}_{A}=\hat{\mathfrak{R}}_{A o}+\left.\frac{\partial \hat{\mathfrak{R}}_{A}}{\partial \hat{W}}\right|_{0}\left(\hat{W}_{2}-\hat{W}_{2 o}\right)+\left.\frac{\partial \hat{\mathfrak{R}}_{A}}{\partial \hat{\mu}_{A m}}\right|_{o}\left(\hat{\mu}_{A m}-\hat{\mu}_{A m o}\right)+\left.\frac{\partial \hat{\mathfrak{R}}_{A}}{\partial \hat{r}_{4}}\right|_{o}\left(\hat{r}_{4}-\hat{r}_{4 o}\right)+\left.\frac{\partial \hat{\mathfrak{R}}_{A}}{\partial \hat{r}_{3}}\right|_{o}\left(\hat{r}_{3}-\hat{r}_{3 o}\right)$

This result can be rearranged and written as

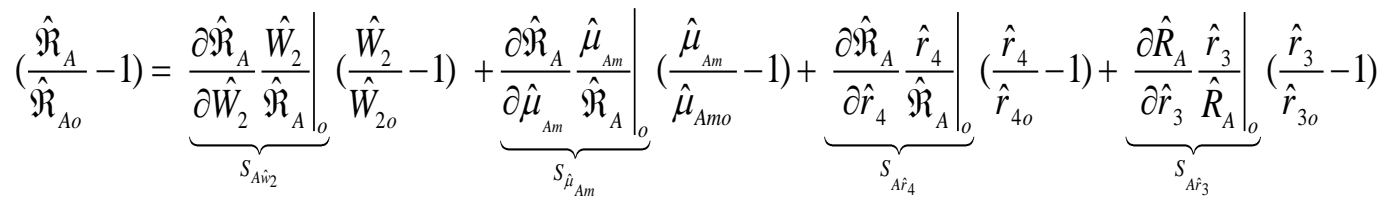

where the sensitivity coefficients are given by,

$$
S_{A \hat{w}_{2}}=+1, S_{\hat{\mu}_{A m}}=-1, S_{A \hat{r}_{4}}=\left.\frac{-2 \hat{r}_{4}^{2}}{\hat{r}_{4}^{2}-\hat{r}_{3}^{2}}\right|_{o}, S_{A \hat{r}_{3}}=\left.\frac{2 \hat{r}_{3}^{2}}{\hat{r}_{4}^{2}-\hat{r}_{3}^{2}}\right|_{o}
$$

For the nondimensional reluctance $\hat{\mathfrak{R}}_{B}$;

$$
\hat{\mathfrak{R}}_{B}=\hat{\mathfrak{R}}_{B o}+\left.\frac{\partial \hat{\mathfrak{R}}_{B}}{\partial \hat{\mu}_{B m}}\right|_{o}\left(\hat{\mu}_{B m}-\hat{\mu}_{B m o}\right)+\left.\frac{\partial \hat{\mathfrak{R}}_{B}}{\partial \hat{r}_{4}}\right|_{o}\left(\hat{r}_{4}-\hat{r}_{4 o}\right)+\left.\frac{\partial \hat{\mathfrak{R}}_{B}}{\partial \hat{r}_{3}}\right|_{o}\left(\hat{r}_{3}-\hat{r}_{3 o}\right)+\left.\frac{\partial \hat{\mathfrak{R}}_{B}}{\partial \hat{W}_{1}}\right|_{o}\left(\frac{\hat{W}_{1}}{\hat{W}_{10}}-1\right)
$$

This result can be rearranged and written as

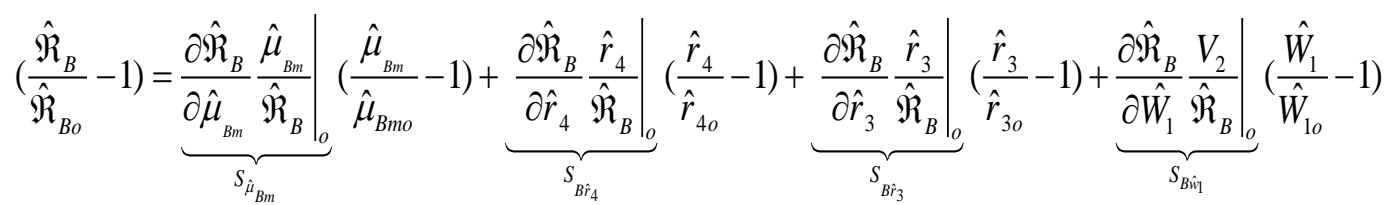

where the sensitivity coefficients are given by

$$
S_{\hat{\mu}_{B m}}=S_{B \hat{w}_{1}}=-1, S_{B \hat{r}_{4}}=1 /\left.\ln \left(\frac{\hat{r}_{4}}{\hat{r}_{3}}\right)\right|_{o}, S_{B \hat{r}_{3}}=-1 /\left.\ln \left(\frac{\hat{r}_{4}}{\hat{r}_{3}}\right)\right|_{o}
$$

For the nondimensional reluctance $\hat{\mathfrak{R}}_{C}$; 


$$
\hat{\mathfrak{R}}_{C}=\hat{\mathfrak{R}}_{C o}+\left.\frac{\partial \hat{\mathfrak{R}}_{C}}{\partial \hat{h}}\right|_{o}\left(\hat{h}-\hat{h}_{o}\right)+\left.\frac{\partial \hat{\mathfrak{R}}_{C}}{\partial \hat{\mu}_{C m}}\right|_{o}\left(\hat{\mu}_{C m}-\hat{\mu}_{C m o}\right)+\left.\frac{\partial \hat{\mathfrak{R}}_{C}}{\partial \hat{r}_{2}}\right|_{o}\left(\hat{r}_{2}-\hat{r}_{2 o}\right)+\left.\frac{\partial \hat{\mathfrak{R}}_{C}}{\partial \hat{r}_{1}}\right|_{o}\left(\hat{r}_{1}-\hat{r}_{1 o}\right)
$$

This result can be rearranged and written as

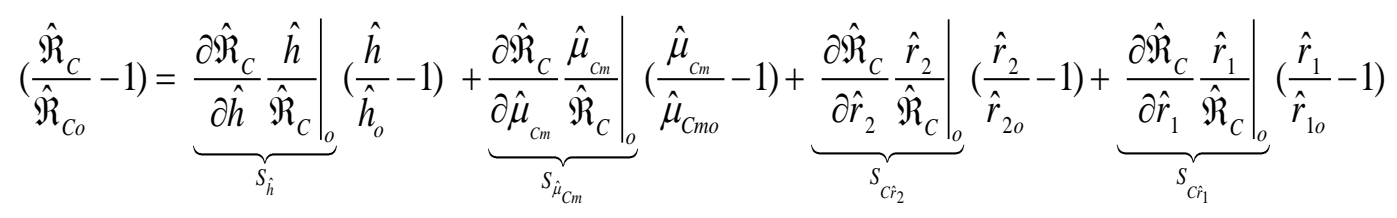

where the sensitivity coefficients are given by

$$
S_{\hat{\mu}_{C m}}=-1, S_{\hat{h}}=+1, S_{C \hat{r}_{1}}=\left.\frac{2 \hat{r}_{1}^{2}}{\left(\hat{r}_{2}^{2}-\hat{r}_{1}^{2}\right)}\right|_{o}, S_{C \hat{r}_{2}}=\left.\frac{-2 \hat{r}_{2}^{2}}{\left(\hat{r}_{2}^{2}-\hat{r}_{1}^{2}\right)}\right|_{0}
$$

For the nondimensional reluctance $\hat{\mathfrak{R}}_{D}$;

$$
\hat{\mathfrak{R}}_{D}=\hat{\mathfrak{R}}_{D o}+\left.\frac{\partial \hat{\mathfrak{R}}_{D}}{\partial \hat{w}_{3}}\right|_{o}\left(\hat{W}_{3}-\hat{W}_{3 o}\right)+\left.\frac{\partial \hat{\mathfrak{R}}_{D}}{\partial \hat{\mu}_{D m}}\right|_{o}\left(\hat{\mu}_{D m}-\hat{\mu}_{D m o}\right)+\left.\frac{\partial \hat{\mathfrak{R}}_{D}}{\partial \hat{r}_{2}}\right|_{o}\left(\hat{r}_{2}-\hat{r}_{2 o}\right)+\left.\frac{\partial \hat{\mathfrak{R}}_{D}}{\partial \hat{r}_{2}}\right|_{o}\left(\hat{r}_{1}-\hat{r}_{1 o}\right)
$$

This result can be rearranged and written as,

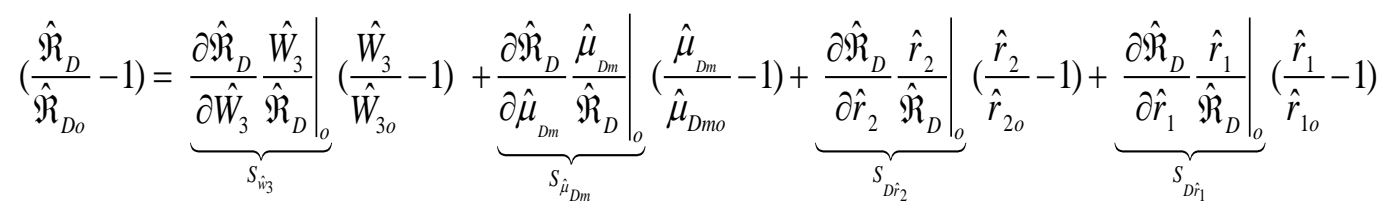

where the sensitivity coefficients are given by,

$$
S_{D \hat{W}_{3}}=+1, S_{\hat{\mu}_{D m}}=-1, S_{D \hat{r}_{2}}=\left.\frac{-2 \hat{r}_{2}^{2}}{\left(\hat{r}_{2}^{2}-\hat{r}_{1}^{2}\right)}\right|_{0}, S_{D \hat{r}_{1}}=\left.\frac{+2 \hat{r}_{2}^{2}}{\left(\hat{r}_{2}^{2}-\hat{r}_{1}^{2}\right)}\right|_{0}
$$

The sensitivity coefficient with the large absolute value will indicate that a plus or minus percent change in that parameter will create the largest impact on the torque capacity.

\subsection{Summary}

In this chapter, the mathematical models of conventional brake, MRF brake and 
electromagnetic circuit are presented. Nondimensional analysis is used to generalize the mathematical modeling. Next, sensitivity analysis using first order Taylor series is used to obtain sensitivity coefficients of all design parameters. Optimal designs of conventional and MR fluid brakes using Taguchi method will be presented in the next chapter. 


\section{CHAPTER 4. OPTIMAL DESIGN USING TAGUCHI METHOD}

\subsection{General}

Optimal design or robust design can be defined as the engineering technique that used to minimize the sensitivity of a product (specific design) to variations of design parameters (internal and external parameters) [109, 110]. Robust design is basically depending on some statistical models which developed to design of experiments. The main objective of design of experiments is to identify an optimal setting of design parameters which achieve an optimal design [111]. In this chapter, the Taguchi approach, design of experiments, and how design parameters and its optimal levels are presented.

\subsection{Experimental Design}

\subsubsection{Design of Experiments}

Design of experiment (or test for design) is an efficient method used to test several design parameters of a specific design. Design of experiments can be defined as a set of tests involving changing some design parameters with some levels and their effect on output(s) design parameter(s). The main purpose of design of experiments is to get a wide range of information about input and output design parameters and how they interact of each other. In general, design of experiments can be divided into three groups, factorial design, response surface design, and evolutionary operation $[112,113]$. The engineering solving scheme is shown in Fig. 4-1. Engineering problem solving steps start with a problem definition by studying the relationship between input and output design parameters. Output design parameter(s) is one of three desired outcomes: maximizing 
output design parameter(s), making design response as close as possible to a target point, and the third is minimizing output design.

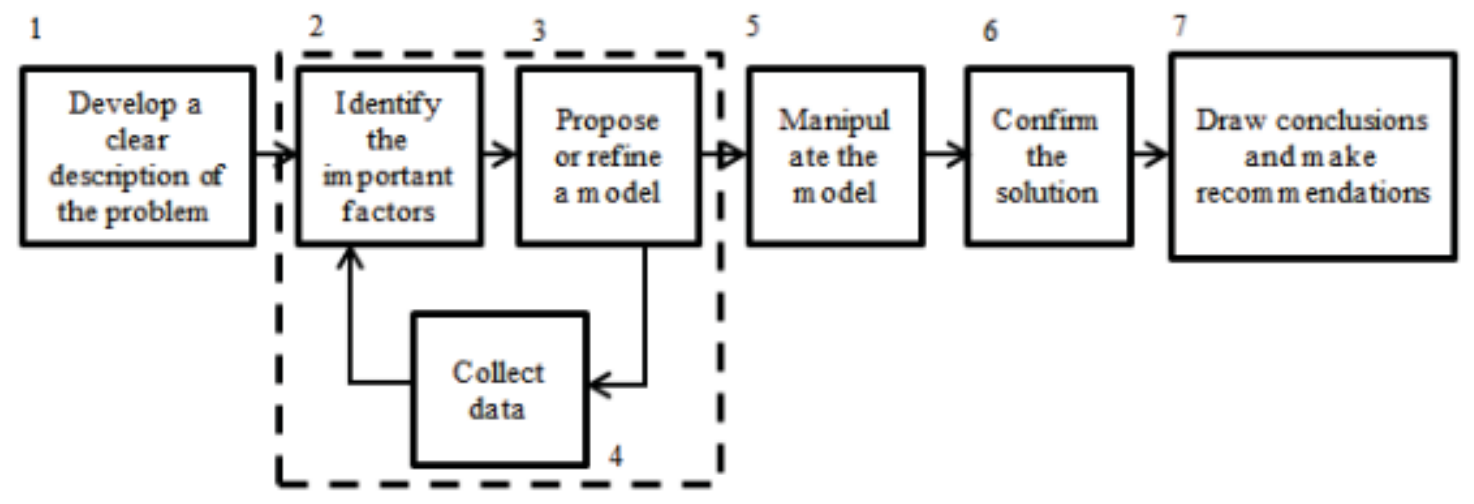

Figure 4-1. Engineering problem solving scheme [113]

The second step is to identify the important factors of a design using specific guessing method such as brainstorm and critical thinking $[114,115]$. The next step is to propose a model and collect data continuously along with parameters identifications. For more information about solving scheme refer to [113].

In this work, factorial design is used to predict the optimum design of magnetorheological fluid brake (MRF). Factorial design can be divided into two parts, full and fractional factorial design. For any engineering problem, a designer should identify the important factors (design parameters) which need to be changed in each experiment and achieving design goal. In other words, the main purpose of design of experiments is to investigate effect of several design parameters by changing them simultaneously rather than changing them separately $[116,117]$. That means factorial experimental design is used to identify sensitivity of each design parameters and then obtain the best combination of design parameters that achieve an optimal design 
performance. To understand how design of experiments works it important to go through its fundamental principles.

\subsubsection{Fundamentals of Design of Experiments}

According to [118], there are five fundamental principles for design of experiments; randomization, replication, blocking, orthogonality, and factorial experimentation. These will be discussed below.

Randomization is the first principle for any design of experiment which implies that every design or any distribution of factors has same probability. Randomization process is very important in a comparative study to compare effect of each design parameters on design performance. In addition, randomization process removes bias (uncontrollable) and other variation sources.

Replication process can be used to increase the width of confidence interval and controlling the uncertainty in design response. Replication can be carried out by repeating same experiment exactly at same conditions [118]. Finally, replication increases a precision of experiments. Another way to increase experiments precision can be achieved by using blocking principle and reducing or removing effect of uncontrollable design parameters (noise factors).

Blocking process is another way of controlling some parameters variations that reduces factors variations and then reducing bias (resulting from variations of noise factors) and making errors as small as possible [119].

Orthogonality principle is very important in design of experiment. In general, orthogonality means that factors in each experiment vary independently of each other. 
The main advantage of that, the output of all experiments can be identified using their average difference. Orthogonal principle will be given in details in the next sections. Finally, factorial experimentation is a way of changing all design parameters simultaneously and studying their effect.

\subsection{Taguchi Design and Orthogonal Array}

Taguchi suggested using specific mathematical tools (orthogonal arrays) to find the required number of factors (design parameters) and associated levels for each experimental design [122]. Number of experiments depends on number of design parameters and corresponding levels (practically, values of each design variables). For example, two factors of two levels each needs $2^{2}=4$ experiments while six factors and five levels each needs $5^{6}=15625$ experiment to study effect of design parameters and find the best design. This mode of design called full factorial design of experiment because it considers achieving all the required experiments for testing by changing only one design parameter at a time. However, Taguchi suggested using special orthogonal arrays that using a smaller number of experiments (fraction) instead of the total number of experiments due to use full factorial design.

The way of using a smaller number of experiments using orthogonal arrays called fractional factorial design of experiments in which design parameters vary simultaneously instead of independently [123]. The main advantage of the fractional design is to reduce the required number of experiments. For example, Taguchi used 16 experiments only to study a specific design of experiments of five factors with four levels each. However, in the full factorial design, a designer needs to achieve $4^{5}=1024$ 
experiments. $\mathrm{L}_{16}$ refers to an orthogonal array of 16 experiments. Number of design parameters (factors) and their values (levels) are different from design to another. Therefore, Taguchi developed 18 standard orthogonal arrays. However, standard orthogonal array can be changed to fit a specific design.

Some of standard orthogonal array are shown in Fig. 4-2. The number of rows is the minimum number of required experiments, while the columns represent number of design parameters (factors). Factors can be divided into controllable and uncontrollable design parameters.

\begin{tabular}{|c|c|c|c|}
\hline \multirow{2}{*}{ Run } & \multicolumn{3}{|c|}{ Columns } \\
\cline { 2 - 4 } & 1 & 2 & 3 \\
\hline 1 & 1 & 1 & 1 \\
2 & 1 & 2 & 2 \\
3 & 2 & 1 & 2 \\
4 & 2 & 2 & 1 \\
\hline
\end{tabular}

\begin{tabular}{|c|c|c|c|c|c|}
\hline \multirow{2}{*}{ Run } & \multicolumn{5}{|c|}{ Columns } \\
\cline { 2 - 6 } & 1 & 2 & 3 & 4 & 5 \\
\hline 1 & 1 & 1 & 1 & 1 & 1 \\
2 & 2 & 2 & 2 & 2 & 1 \\
3 & 1 & 1 & 2 & 2 & 2 \\
4 & 2 & 2 & 1 & 1 & 2 \\
5 & 1 & 2 & 1 & 2 & 3 \\
6 & 2 & 1 & 2 & 1 & 3 \\
7 & 1 & 2 & 2 & 1 & 4 \\
8 & 2 & 1 & 1 & 2 & 4 \\
\hline
\end{tabular}

\begin{tabular}{|c|c|c|c|c|}
\hline \multirow{2}{*}{ Run } & \multicolumn{4}{|c|}{ Columns } \\
\cline { 2 - 5 } & 1 & 2 & 3 & 4 \\
\hline 1 & 1 & 1 & 1 & 1 \\
2 & 1 & 2 & 2 & 2 \\
3 & 1 & 3 & 3 & 3 \\
4 & 2 & 1 & 2 & 3 \\
5 & 2 & 2 & 3 & 1 \\
6 & 2 & 3 & 1 & 2 \\
7 & 3 & 1 & 3 & 2 \\
8 & 3 & 2 & 1 & 3 \\
9 & 3 & 3 & 2 & 1 \\
\hline
\end{tabular}
a) $\mathrm{L}_{4}(3 * 4)$
b) $\mathrm{L}_{8}(3 * 4)$
c) $\mathrm{L}_{9}(3 * 9)$

Figure 4-2. Some of standard orthogonal arrays.

Orthogonal arrays are two dimensional matrices and its main characteristic is the orthogonality of its column. In other words, column of an orthogonal array must be mutual orthogonal and that implies level combination for all factor are presented in an equal time through the orthogonal array [124].

\subsection{Optimization Design Characteristics}

Taguchi approach is a powerful method to study effect of design variables (design 
parameters) on the design goal (design output/s) and carrying out a best design of a problem under study. Design characteristic to be perfected is the first step of Taguchi method of optimization. It easily means an identification process whether optimization process considers maximization, minimization, or achieving a target.

In general, Taguchi described design and quality characteristics by the deviations of a design response from a desired point (target point). The variations refer to quality losses which is proportional to the difference between the actual and desired point [125, $126,127]$.

The backbone of Taguchi design of experiments approach is reducing the variations of the output parameter(s) and quality losses to lower levels and leading the output to a target. Based on that, Taguchi developed three quality loss functions depending on the square of variations mentioned above. These functions vary nonlinearly instead of step change behavior of traditional loss functions. Figure 4-3 [128] shows both traditional and Taguchi approaches.

\section{$L(y)$ Quality Loss}

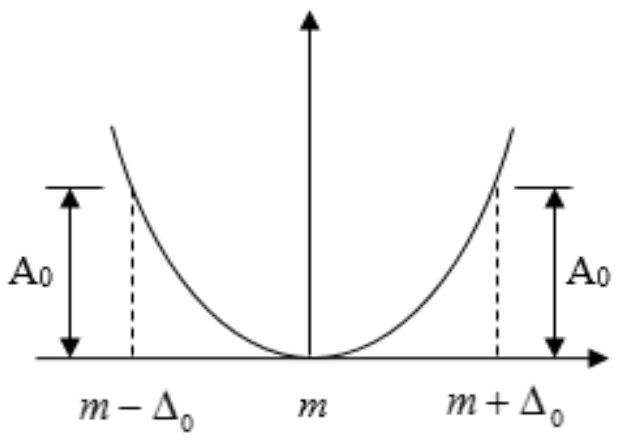

a) Taguchi loss function 


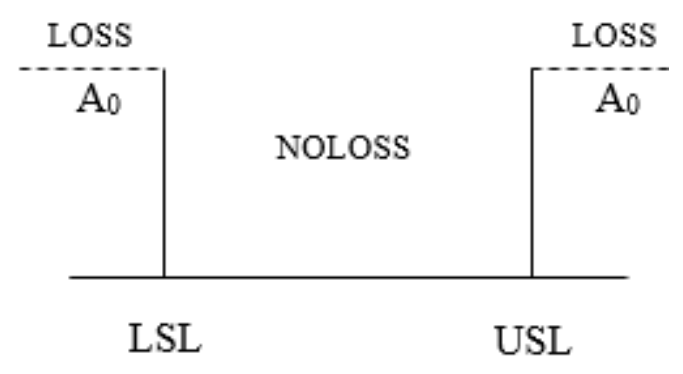

b) Traditional approach

Figure 4-3. Traditional approach and Taguchi loss function [128]

\subsection{Quality Loss Functions}

Three quality loss functions were developed; nominal the best, smaller the better, and larger the better. The general form of loss functions can be given by,

$$
L(y)=k(y-m)^{2}
$$

where $\mathrm{L}$ is the loss function, $\mathrm{y}$ is the objective response, $\mathrm{m}$ is the target, and $\mathrm{k}$ is a coefficient depends on target and optimization process. $k$ is given as $k=A_{0} / \Delta^{2}$ where $A_{0}$ and $\Delta$ are showed in Fig. 4-3 [129]. The philosophy beyond nonlinear behavior of loss functions reveals that losses increases with increasing variations of the output from the target and decreases as it close to the target. That means no losses at the target. Simple comparison between traditional and Taguchi loss functions reveals that Taguchi loss functions is continuous functions while a suddenly changing for the traditional approach.

When a desired value is a target, the loss function called nominal the best. If a desired design value is required to increase (Braking torque in this study) then the quality loss function called larger the better. However, it is called smaller the better in case of 
desired value needed to be minimized as shown in Fig. 4-4 [130].
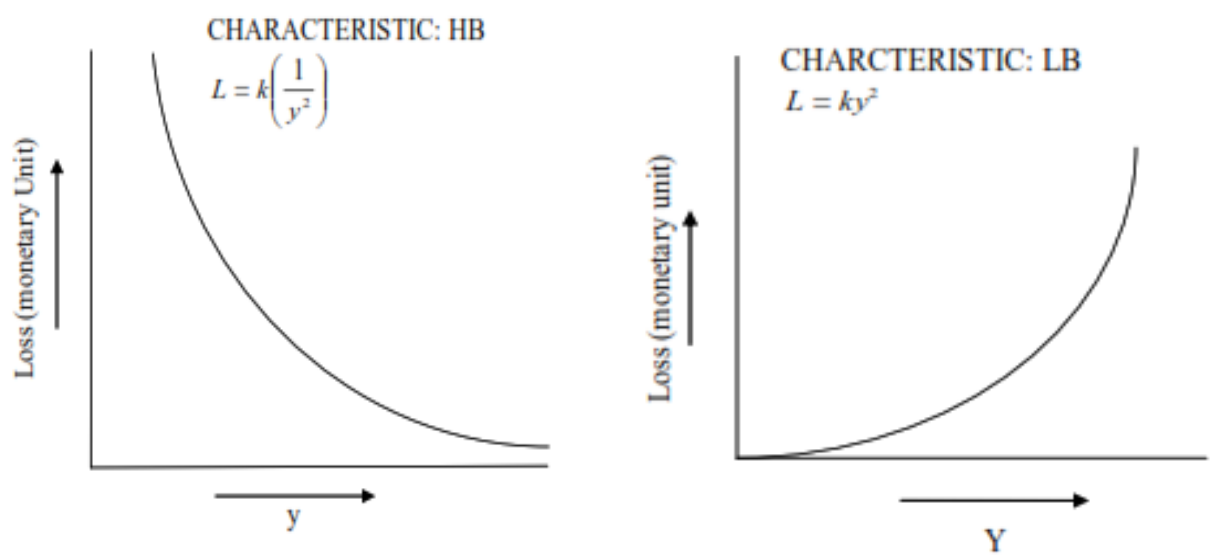

Figure 4-4. Taguchi loss function, smaller the better and larger the better

\subsection{Signals to Noise Ratio and Quality Function}

The next step is to find the optimal levels of control factors that offer an optimal value of design response. Design performance is measured statistically using signal to noise ratio $(\mathrm{S} / \mathrm{N})$ which easily be the ratio of the mean value to the standard deviation. Signal to noise ratio is a good measurement of quality variation, so that the greater value of $\mathrm{S} / \mathrm{N}$ is perfect to make the quality variation around the desired value as small as possible $[126,129]$. In general, there are three standard signals to noise ratios $(\mathrm{S} / \mathrm{N})$ depending on what kind of quality loss function that used in the design. They are described in the following paragraphs [130, 131, 132],

1. Smaller the Better

In this case, the desired output is needed to be as small as possible. For example, in a combustion engine, fuel consumption is desired to be lower. The signal to noise ratio can be given as follows, 


$$
S / N=-10 \log \left(\sum_{i=1}^{n} \frac{y_{i}^{2}}{n}\right)
$$

where $y_{i}$ is the performance characteristic and $n$ is the number of repetition (number of trials).

\section{Nominal the Best}

In this case, the desired output is needed to be very close to a target. For example, the dimensions of piston and piston rings of a hydraulic actuator are needed to be exactly the same as the target dimensions (desired values). The signal to noise ratio for nominal the best can be given as follows,

$$
S / N=10 \log \left(\frac{\bar{Y}}{S}\right)^{2}
$$

where

$$
\begin{gathered}
\bar{Y}=\sum_{i=1}^{n} \frac{y_{i}^{2}}{n} \\
S^{2}=\sum_{i=1}^{n} \frac{\left(y_{i}-\bar{Y}\right)^{2}}{n-1}
\end{gathered}
$$

\section{Larger the Better}

In this case, the desired output is needed to be large as possible. For example, Hydraulic pump efficiency and braking torque are needed to be maximized to get higher performance for a specific combination of design parameters. The signal to noise ratio for larger the better can be given as follows,

$$
S / N=-10 \log \left(\sum_{i=1}^{n} \frac{1}{n} \frac{1}{y_{i}^{2}}\right)
$$

Theoretically speaking, signal to noise ratio is a statistical rule combines two characteristics (mean value and the variance around the mean value) into one value [130]. 
Higher signal to noise ratio implies a best performance with the higher quality and very small variations in noise factor $[131,132]$.

\subsection{Table of Response}

Considering signal to noise ratios from the orthogonal array calculations, best value of the design objective mainly corresponds to the largest signal to noise ratio. Table of response shows effect of each level for all factors on the design objective (nondimensional braking torque, for example). Using table of response, one can identify the experiment that gives the best result regarding design performance. Signal to noise ratios for each factor at each level are adding together and divided by levels number to obtain the mean value. For example, for $\mathrm{L}_{4}$ orthogonal array, table of response can be calculated as shown in Fig. 4-5.

A simple comparison between the means for each separate factor reveals the largest value among them, which finds the optimal level for that factor. For example, the greatest values for mean are shaded (red color) for all factors, that means the best levels are the first level for the factor A and the second level for the second and third factors (B and C), respectively.

\begin{tabular}{|c|c|c|c|c|c|c|c|c|c|}
\hline \multirow{2}{*}{$\begin{array}{l}\text { Test } \\
\text { No. }\end{array}$} & \multicolumn{5}{|c|}{ Factors (Design variables) } & \multirow[t]{2}{*}{ Level } & \multicolumn{3}{|c|}{ Factors (Design variables) } \\
\hline & A & B & $\mathrm{C}$ & Output & $(S / N)_{i}$ & & A & B & $\mathrm{C}$ \\
\hline 1 & 1 & 1 & 1 & (Out) 1 & $(S / N)_{1}$ & 1 & $\left.[S / N)_{1}+(S / N)_{2}\right] / 2$ & $\left.[S / N)_{1}+(S / N)_{3}\right] / 2$ & $\left.[S / N)_{1}+(S / N)_{4}\right] / 2$ \\
\hline 2 & 1 & 2 & 2 & (Out)2 & $(S / N)_{2}$ & 2 & $\left.[S / N)_{3}+(S / N)_{4}\right] / 2$ & & $\left.S / N)_{2}+(S / N)_{3}\right]$ \\
\hline 3 & 2 & 1 & 2 & $(\mathrm{Out})_{3}$ & $(S / N)_{3}$ & & & & \\
\hline 4 & 2 & 2 & 1 & (Out)4 & $(S / N)_{4}$ & & & & \\
\hline
\end{tabular}

Figure 4-5. L4 orthogonal array and their table of response 


\subsection{Analysis of Variance (ANOVA)}

Analysis of variation of the resulted $\mathrm{S} / \mathrm{N}$ values is the next step in Taguchi approach. First of all, analysis of variance is considered as a good statistical tool using to identify the significance of each design parameter [132]. Theoretically speaking analysis of variance detects the differences of the mean performance by distributing and dividing the total variations into its original components for each separate design parameters [129]. Analysis of variance (ANOVA) is basically compares the mean sequence values and its associated errors.

Summation of squares deviational values form the mean value of signal to noise ratio is calculated using,

$$
S S=\sum_{i=1}^{n}\left(n_{i}-n_{m}\right)^{2}
$$

where $n$ is the number of experiments, $n_{i}$ is the means signal to the noise ratio for $i^{\text {th }}$ experiment, and $n_{\mathrm{m}}$ is the mean of signal to noise ratio for all experiments. An Effect of design parameters of $95 \%$ of confidence level is considered for ANOVA calculations. Analysis of variance contains several functions and quantities like squares of the means (variance), degrees of freedom (D.F) which represents (number of levels-1), ratio of variance $(F)$ which is the ratios of mean square error to the residual error which give an indication about the factor contribution as well, and percent contribution. All of these quantities are tabulated in the analysis of variance table as shown in Table 4-1. Column $\mathrm{F}$, is the ratios of mean square error to the residual error which gives a sign about the factor contribution as well. Contribution ratio of each parameter indicates the extent at which that parameter has affected the response. 
Table 4-1. Analysis of variance

\begin{tabular}{|l|l|l|l|l|}
\hline \multicolumn{4}{|l|}{ Analysis of variance of S/N } \\
\hline $\begin{array}{l}\text { Factors } \\
\text { (design } \\
\text { parameters) }\end{array}$ & $\begin{array}{l}\text { Degrees of } \\
\text { freedom (D.F) }\end{array}$ & $\begin{array}{l}\text { Means } \\
\text { squares } \\
\text { (SS) }\end{array}$ & $\begin{array}{l}\text { Factor } \\
\text { (F) }\end{array}$ & Contribution \\
\hline A & & & & \\
\hline B & & & & \\
\hline C & & & & \\
\hline Total & & & & \\
\hline
\end{tabular}

\subsection{Taguchi Application for Conventional and Magnetorheological Fluid Brakes}

\subsubsection{Conventional Drum Brake}

In this section, an optimal design of the conventional drum brake using Taguchi approach is presented. The first step is to find a proper orthogonal array. As seen in Chapter 3, four nondimensional parameters are used for the sensitivity analysis. These factors represent the four factors in the orthogonal array. Four factors and three levels each can be used to do the optimization process. The minimum number of the experiments can be given depends on number of factors and levels as $N=F(L-1)+1$ where $N$ is the minimum number of experiments ( 9 in the current work), $L$ is the number of levels, $F$ is number of factors. The degree of freedom can be given by $(L-1)$. In this work, $L_{9}$ four factors- three levels orthogonal array is used as shown in Table 4-2. Table 4-3 shows the equivalent numerical values of the orthogonal array and design objective for each experiment. The last column is the signal to noise ratio for larger the better approximation. The next step is considering the signal to noise ratio from the orthogonal 
orthogonal array calculations, to find the equivalent table response to show effect of each level for all factors on the design objective (non-dimensional torque). Table 4-4 shows signal to noise ratio of each factors and associated levels.

Table 4-2. $L_{9}$ Orthogonal Array

Table 4-3. Numerical Values of $L_{9}$

\begin{tabular}{|c|c|c|c|c|}
\hline \multirow{2}{*}{ Test No. } & \multicolumn{4}{|l|}{ Factors (Design variables) } \\
\cline { 2 - 5 } & F1 & F2 & F3 & F4 \\
\hline 1 & 1 & 1 & 1 & 1 \\
\hline 2 & 1 & 2 & 2 & 2 \\
\hline 3 & 1 & 3 & 3 & 3 \\
\hline 4 & 2 & 1 & 2 & 3 \\
\hline 5 & 2 & 2 & 3 & 1 \\
\hline 6 & 2 & 3 & 1 & 2 \\
\hline 7 & 3 & 1 & 3 & 2 \\
\hline 8 & 3 & 2 & 1 & 3 \\
\hline 9 & 3 & 3 & 2 & 1 \\
\hline
\end{tabular}

\begin{tabular}{|c|c|c|c|c|c|c|}
\hline \multirow{2}{*}{ Test No. } & \multicolumn{6}{|l|}{ Factors (Design variables) } \\
\cline { 2 - 7 } & $\hat{a}$ & $\hat{b}$ & $\hat{c}$ & $\gamma$ & $\hat{T}$ & $\mathrm{~S} / \mathrm{N}$ \\
\hline 1 & 10 & 4 & 0 & 45 & 3.447 & 10.749 \\
\hline 2 & 10 & 5 & 0.5 & 90 & 2.450 & 7.957 \\
\hline 3 & 10 & 6 & 1 & 135 & 2.081 & 6.366 \\
\hline 4 & 12.5 & 4 & 0.5 & 135 & 4.576 & 13.209 \\
\hline 5 & 12.5 & 5 & 1 & 45 & 2.577 & 8.221 \\
\hline 6 & 12.5 & 6 & 0 & 90 & 2.806 & 8.960 \\
\hline 7 & 15 & 4 & 1 & 90 & 4.233 & 12.527 \\
\hline 8 & 15 & 5 & 0 & 135 & 4.767 & 13.567 \\
\hline 9 & 15 & 6 & 0.5 & 45 & 2.803 & 9.972 \\
\hline
\end{tabular}

Table 4-4. Signal to noise ratio of each factors and associated levels.

\begin{tabular}{|c|c|c|c|c|}
\hline \multirow{2}{*}{ Levels } & \multicolumn{4}{|c|}{ Factors (Design variables) } \\
\cline { 2 - 5 } & $\hat{a}$ & $\hat{b}$ & $\hat{c}$ & $\gamma$ \\
\hline 1 & 8.356 & $\mathbf{1 2 . 1 6 1}$ & $\mathbf{1 1 . 0 9 1}$ & 9.313 \\
\hline 2 & 10.129 & 9.913 & 10.045 & 9.814 \\
\hline 3 & $\mathbf{1 1 . 6 8 7}$ & 8.099 & 9.037 & $\mathbf{1 1 . 0 4 6}$ \\
\hline
\end{tabular}

A simple comparison between factors reveals the largest value among them, which finds the optimal level for that factor. For example, the greatest values for mean are shaded 
(gray color) for all factors, that means the best levels are the third, first, first, and the third level for the first, second, third, and the fourth factors, respectively.

The next step is the analysis of variance tables. The main purpose of analysis of variance is to obtain which of design parameters is significant. Effect of design parameters of $95 \%$ of confidence level is considered for ANOVA calculations. Table 4-5 presents ANOVA calculations.

Table 4-5. Analysis of variance for conventional frictional brake

\begin{tabular}{|c|c|c|c|c|}
\hline \multicolumn{5}{|c|}{ Analysis of variance of S/N } \\
\hline Factors & $\begin{array}{c}\text { Degrees of } \\
\text { freedom (D.F) }\end{array}$ & $\begin{array}{c}\text { Means squares } \\
\text { (SS) }\end{array}$ & Factor (F) & Contribution \\
\hline$\hat{a}$ & 2 & 22.219 & 1.747 & 31.678 \\
\hline$\hat{b}$ & 2 & 33.123 & 2.604 & 47.225 \\
\hline$\hat{c}$ & 2 & 8.438 & 0.664 & 12.029 \\
\hline$\gamma$ & 2 & 6.306 & 0.5 & 9.069 \\
\hline Total & & 70.1395 & & $100 \%$ \\
\hline
\end{tabular}

The last column is the contribution of each parameter with $\mathrm{F}$ values for all sources of variability. The percentage contribution is the corresponding value divided by total summation of squares. Contribution ratio of each parameter indicates the extent at which that design variable has affected the response. One can note that $\hat{b}$ has the largest contribution of $47.225 \%$. The second effective parameter is the horizontal central distance $\hat{a}$ of $31.678 \%$. The third is the vertical central distance $\hat{c}$ of $12.029 \%$. The fourth is the drum angle $\gamma$ of $9.069 \%$. These results give the designer a good indication 
about getting the optimal design of the drum brake and what factor he need to alter to increase the nondimensional braking torque.

\subsubsection{Comments}

The signal to noise ratio for each experiment is calculated considering larger the better loss function. As mentioned previously, the design objective is to maximize the non-dimensional braking torque. The optimal objective is just corresponding to the optimum parameters values (levels) which show larger signal to noise ratio $(\mathrm{S} / \mathrm{N})$ as shown in Fig. 4-6 below.

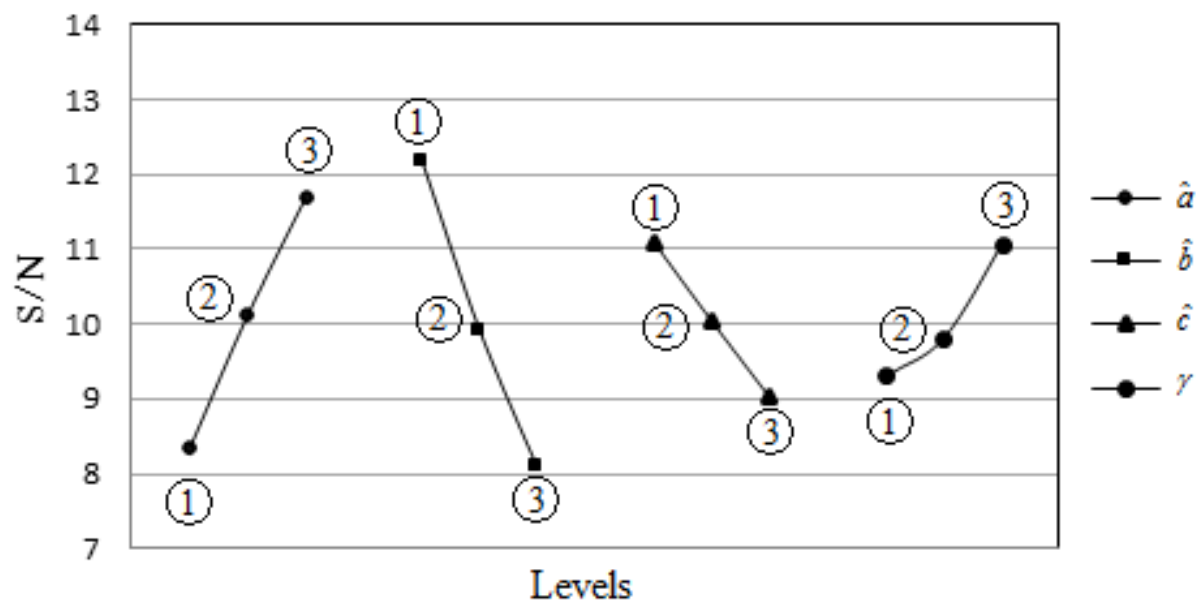

Figure 4-6. Signal to noise ratio and best levels for the factors

It is noted that signal to noise ratio is increases with both nondimensional factors $\hat{a}$ and $\gamma$ and decreases with $\hat{b}$ and $\hat{c}$, respectively. From Fig. 4-6, the optimum design the conventional drum brake for maximum nondimensional braking torque can be achieved by just select the levels that give the larger signal to noise ratio value. The optimum levels for optimal signal to noise ratio are 3-1-1-3 for $\hat{a}, \hat{b}, \hat{c}$, and $\gamma$, respectively. 
Finally, it noteworthy that both results of sensitivity analysis for the nondimensional parameters using Taylor approximation and Taguchi shows same trend.

\subsubsection{Magnetorheological Fluid Brake}

In this section, an optimal design of the MR fluid brake is presented. The first step is to find a proper orthogonal array. As mentioned in Ch. 3, off and on field braking torque are created in the MR fluid brake. Each one is represented by four nondimensional design parameters. Similar to conventional frictional brake, Taguchi method is applied for both braking torques separately using the same orthogonal array.

\subsubsection{Off-Field Braking Torque}

Four factors and three levels each can be used to do the optimization process. $L_{9}$ four factors- three levels orthogonal array is used as shown in Table 4-6.

Table 4-6. Numerical Values of $L_{9}$ for $\hat{T}_{p}$.

\begin{tabular}{|c|c|c|c|c|c|c|}
\hline \multirow{2}{*}{ Test No. } & \multicolumn{6}{|c|}{ Factors (Design variables) } \\
\cline { 2 - 7 } & $\hat{r}_{i}$ & $\hat{w}$ & $\hat{\mu}$ & $\hat{h}$ & $\hat{T}_{p}$ & $\mathrm{~S} / \mathrm{N}$ \\
\hline 1 & 0.5 & 0.9 & 0.9 & 0.8 & 0.949 & -0.452 \\
\hline 2 & 0.5 & 1 & 1 & 0.9 & 1.042 & 0.354 \\
\hline 3 & 0.5 & 1.1 & 1.1 & 1 & 1.134 & 1.095 \\
\hline 4 & 0.6 & 0.9 & 1 & 1 & 0.783 & -2.120 \\
\hline 5 & 0.6 & 1 & 1.1 & 0.8 & 1.197 & 1.560 \\
\hline 6 & 0.6 & 1.1 & 0.9 & 0.9 & 0.957 & -0.377 \\
\hline 7 & 0.7 & 0.9 & 1.1 & 0.9 & 0.836 & -1.557 \\
\hline 8 & 0.7 & 1 & 0.9 & 1 & 0.684 & -3.300 \\
\hline 9 & 0.7 & 1.1 & 1 & 0.8 & 1.045 & 0.381 \\
\hline
\end{tabular}


Table 4-6 shows the equivalent numerical values of the orthogonal array and design objective for each experiment for $\hat{T}_{p}$. The last column is the signal to noise ratio for larger the better approximation. The signal to noise ratio from the orthogonal array calculations of each factor and associated levels is presented in Table 4-7.

Table 4-7. Signal to noise ratio of each factors and associated levels of $\hat{T}_{p}$.

\begin{tabular}{|c|c|c|c|c|}
\hline \multirow{2}{*}{ Levels } & \multicolumn{4}{|c|}{ Factors (Design variables) } \\
\cline { 2 - 5 } & $\hat{r}_{i}$ & $\hat{w}$ & $\hat{\mu}$ & $\hat{h}$ \\
\hline 1 & $\mathbf{0 . 3 3 2}$ & -1.377 & -1.377 & $\mathbf{0 . 4 9 6}$ \\
\hline 2 & -0.313 & -0.462 & -0.462 & -0.527 \\
\hline 3 & -1.492 & $\mathbf{0 . 3 6 6}$ & $\mathbf{0 . 3 6 6}$ & -1.442 \\
\hline
\end{tabular}

A simple comparison between factor reveals the largest value among them, which specifies the optimal level for that factor which shaded (gray color) for all factors. That means the best levels are the first, third, third, and the first level for the first, second, third, and the fourth factors, respectively.

Analysis of variance table for $\hat{T}_{p}$ is shown in Table 4-8. The last column is the contribution of each parameter with $\mathrm{F}$ values for all sources of variability. Contribution ratio of each parameter indicates the extent at which that design variable has affected the response for a specified perturbation around its nominal values. One can note that $\hat{h}$ has the largest contribution of $28.349 \%$. The second effective parameter is the radial ratio $\hat{r}_{i}$ of $25.806 \%$. The results give the designer a good indication about getting the optimal design of the MR fluid brake and what factor he need to alter to increase the corresponding nondimensional braking torque. 
Table 4-8. Analysis of variance for $\hat{T}_{p}$.

\begin{tabular}{|c|c|c|c|c|}
\hline \multicolumn{5}{|c|}{ Analysis of variance of S/N } \\
\hline Factors & $\begin{array}{c}\text { Degrees of } \\
\text { freedom (D.F) }\end{array}$ & $\begin{array}{c}\text { Means squares } \\
(\mathrm{SS})\end{array}$ & Factor (F) & Contribution \\
\hline$\hat{r}_{i}$ & 2 & 6.846 & 0.563 & 25.806 \\
\hline$\hat{w}$ & 2 & 6.081 & 0.500 & 22.922 \\
\hline$\hat{\mu}$ & 2 & 6.081 & 0.500 & 22.922 \\
\hline$\hat{h}$ & 2 & 7.521 & 0.618 & 28.349 \\
\hline Total & & 26.530 & & $100 \%$ \\
\hline
\end{tabular}

\subsubsection{Comments}

As mentioned previously, the design objective is to maximize the non-

dimensional braking torque. The optimal objective is corresponding to the optimum parameters values (levels) which show larger signal to noise ratio $(\mathrm{S} / \mathrm{N})$ as shown in Fig. 4-7 below.

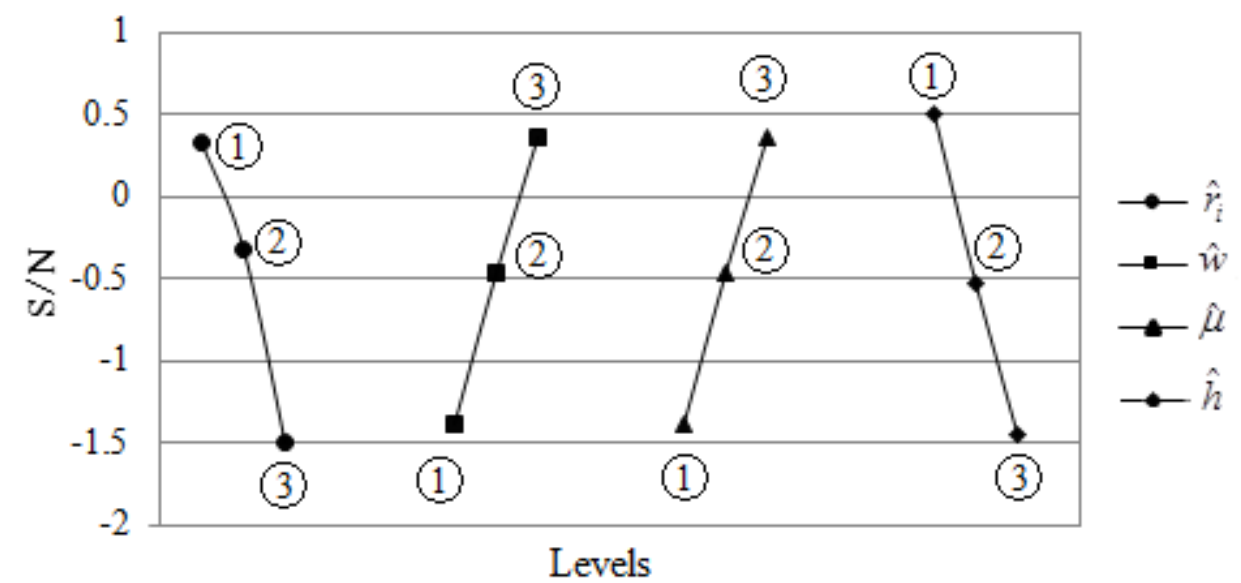

Figure 4-7. Signal to noise ratio and best levels for the factors for $\hat{T}_{p}$.

It is noted that signal to noise ratio is increases with both nondimensional factors $\hat{w}$ and 
$\hat{\mu}$ and decreases with $\hat{r}_{i}$ and $\hat{h}$, respectively. From Fig. 4-7, the optimum design of the MR fluid brake for maximum nondimensional braking torque $\left(\hat{T}_{p}\right)$, can be achieved by just selection the levels that give the larger signal to noise ratio value. The optimum levels for optimal signal to noise ratio are 1-3-3-1. It noteworthy that both results of sensitivity analysis for the nondimensional parameters using Taylor approximation and Taguchi shows same trend as we will see in the subsequent chapters.

\subsubsection{On- Field Braking Torque}

$L_{9}$-four factors- three levels orthogonal array is used as shown in Table 4-9.

Table 4-9. Numerical Values of $L_{9}$ for $\hat{T}_{H}$.

\begin{tabular}{|c|c|c|c|c|c|c|}
\hline \multirow{2}{*}{ Test No. } & \multicolumn{6}{|c|}{ Factors (Design variables) } \\
\cline { 2 - 7 } & $\hat{r}_{i}$ & $\hat{k}$ & $\hat{H}$ & $\hat{\beta}$ & $\hat{T}_{H}$ & $\mathrm{~S} / \mathrm{N}$ \\
\hline 1 & 0.5 & 0.4 & 1.4 & 0.4 & 0.400 & -7.950 \\
\hline 2 & 0.5 & 0.5 & 1.5 & 0.5 & 0.536 & -5.420 \\
\hline 3 & 0.5 & 0.6 & 1.6 & 0.6 & 0.696 & -3.147 \\
\hline 4 & 0.6 & 0.4 & 1.5 & 0.6 & 0.400 & -7.959 \\
\hline 5 & 0.6 & 0.5 & 1.6 & 0.4 & 0.473 & -6.501 \\
\hline 6 & 0.6 & 0.6 & 1.4 & 0.5 & 0.557 & -5.089 \\
\hline 7 & 0.7 & 0.4 & 1.6 & 0.5 & 0.332 & -9.566 \\
\hline 8 & 0.7 & 0.5 & 1.4 & 0.6 & 0.402 & -7.916 \\
\hline 9 & 0.7 & 0.6 & 1.5 & 0.4 & 0.464 & -6.677 \\
\hline
\end{tabular}

Table 4-9 shows the equivalent numerical values of the orthogonal array and design objective for each experiment for $\hat{T}_{H}$. The last column is the signal to noise ratio for 
larger the better approximation. The signal to noise ratio from the orthogonal array calculations of each factor and associated levels is presented in Table 4-10.

Table 4-10. Signal to noise ratio of each factors and associated levels of $\hat{T}_{H}$.

\begin{tabular}{|l|l|l|l|l|}
\hline \multirow{2}{*}{ Levels } & \multicolumn{4}{|l|}{ Factors (Design variables) } \\
\cline { 2 - 5 } & $\hat{r}_{i}$ & $\hat{k}$ & $\hat{H}$ & $\hat{\beta}$ \\
\hline 1 & $\mathbf{- 5 . 5 0 6}$ & -8.492 & -6.985 & -7.043 \\
\hline 2 & -6.517 & -6.612 & -6.685 & -6.692 \\
\hline 3 & -8.053 & $\mathbf{- 4 . 9 7 1}$ & $\mathbf{- 6 . 4 0 5}$ & $\mathbf{- 6 . 3 4 1}$ \\
\hline
\end{tabular}

A comparison between factor reveals the largest value among them, which specifies the optimal level for that factor which shaded (gray color) for all factors. That means the best levels are the first, third, third, and the third level for the first, second, third, and the fourth factor, respectively. Analysis of variance table for $\hat{T}_{H}$ is shown in Table 4-11.

Table 4-11. Analysis of variance for $\hat{T}_{H}$.

\begin{tabular}{|c|c|c|c|c|}
\hline \multicolumn{5}{|c|}{ Analysis of variance of S/N } \\
\hline Factors & $\begin{array}{c}\text { Degrees of } \\
\text { freedom (D.F) }\end{array}$ & $\begin{array}{c}\text { Means squares } \\
(\mathrm{SS})\end{array}$ & Factor (F) & Contribution \\
\hline$\hat{r}_{i}$ & 2 & 13.163 & 9.782 & 33.201 \\
\hline$\hat{k}$ & 2 & 24.826 & 18.448 & 62.618 \\
\hline$\hat{H}$ & 2 & 0.673 & 0.500 & 1.697 \\
\hline$\hat{\beta}$ & 2 & 0.985 & 0.732 & 2.484 \\
\hline Total & & 39.647 & & 100 \\
\hline
\end{tabular}


The last column is the contribution of each parameter with $\mathrm{F}$ values for all sources of variability. One can note that $\hat{k}$ has the largest contribution of $62.618 \%$. The second effective parameter is the radial ratio $\hat{r}_{i}$ of $33.201 \%$. The results give the designer a good indication about getting the optimal design of the MR fluid brake and what factor he need to alter to increase the corresponding nondimensional braking torque.

\subsubsection{Comments}

As mentioned previously, the design objective is to maximize the nondimensional braking torque. The optimal objective is corresponding to the optimum parameters values (levels) which show larger signal to noise ratio $(\mathrm{S} / \mathrm{N})$ as shown in Fig.

4-8. The optimum design of the MR fluid brake for maximum nondimensional braking torque $\left(\hat{T}_{H}\right)$, can be achieved by just select the levels that give the larger signal to noise ratio value. The optimum levels for optimal signal to noise ratio are 1-3-3-3. It noteworthy that both results of sensitivity analysis for the nondimensional parameters using Taylor approximation and Taguchi shows same trend

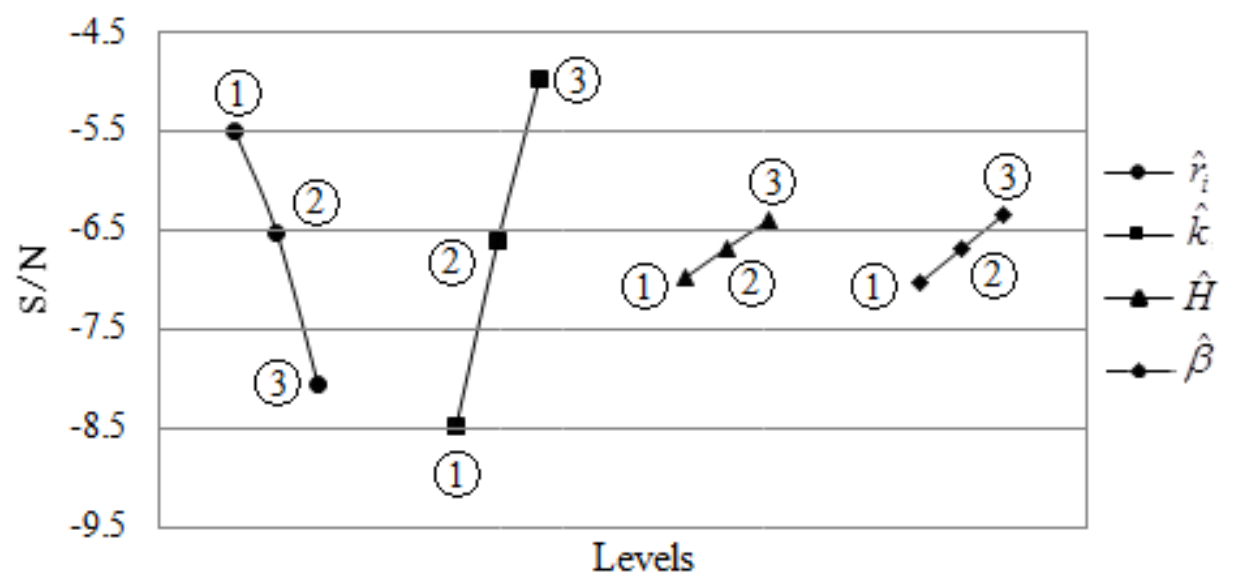

Figure 4-8. Signal to noise ratio and best levels for the factors for $\hat{T}_{H}$. 


\subsection{Conclusions}

Some conclusion ca be drawn from the results and tables as follow:

1- Taguchi method can give a good prediction about the experiment which gives maximum nondimensional braking torque using the orthogonal array approach and the levels of the factors that give best nondimensional braking torque.

2- For the conventional frictional brake, nondimensional braking torque increases with parameters $\hat{a}$ and $\gamma$ and decreases with $\hat{b}$ and $\hat{c}$. This behavior is concluded during sensitivity study in Ch.3 and can be used to generalize the method to obtain an optimal design.

3- For the MF fluid brake, nondimensional on and off-field braking torque increases with $\hat{w}, \hat{\mu}, \hat{k}, \hat{H}$, and $\hat{\beta}$ and decreases with $\hat{h}$ and $\hat{r}_{i}$. This behavior is noticed during sensitivity study in Ch.3 and can be used to generalize the method to obtain an optimal design as well.

4- It noteworthy that both results of sensitivity analysis for the nondimensional parameters using Taylor approximation and factors contributions using Taguchi (nondimensional parameters and the factor are similar to some extent) presents a good coincidence especially for the contribution ratio and optimal levels of the nondimensional groups.

\subsection{Summary}

In this chapter, the basic idea beyond Taguchi approach and design of experiments is presented. General information about fundamentals of design of experiments which contain Taguchi design of experimental approach and orthogonal 
arrays is presented as well. Next, optimization procedures of Taguchi and quality loss functions are explained in details. Next, signal to noise ratio and table of response which is the backbone of Taguchi optimization method are including and their calculations are explained. Finally, optimum levels for the design variables (getting the optimum design) are specified based on table of response. An optimal design of magnetorheological fluid (MRF) brake taking advantage of sensitivity study will be presented in the next chapter. 


\section{CHAPTER 5. MAGNETORHEOLOGICAL FLUID BRAKE OF VARIABLE}

FLLUID THICKNESS (CVFT, NEW OPTIMAL DESIGNS)

\subsection{General}

In order to increase the braking torque, one can change the design parameters in specific ways by making a tradeoff between them. For example, the braking torque can be increased by either increasing the number of frictional surfaces (number of disks) or by using a magnetorheological fluid of high viscosity with smaller fluid thickness. In addition, some people studied the electromagnetic circuit to get an optimal design for magnetorheological fluid brake by increasing the number of coil turns, using a magnetic material of high permeability and low reluctance, increasing the number of electromagnetic coils nearby the activated magnetorheological fluid, and changing bobbin design of the magnetic coil. However, both ways are limited by geometrical space and the heavy weight or may lead to increase the off-filed torque which unfavorable. The main limitations of getting an optimal design by working on electromagnetic circuit design are the magnetic saturation and bottle neck problems (will be discussed in the next section) [133-136, 143-145].

It can be seen from chapter three that the magnetorheological fluid gap or fluid thickness is one of the critical and effective design parameters that may be adjusted in order to have a noticeable impact on braking torque. In this chapter, two new suggested designs of magnetorheological fluid brake are introduced based on changing of the fluid thickness in one and two sides of the rotating disk taking the advantage of sensitivity analysis into the consideration. Brief background about magnetic saturation and hysteresis problems is given in the next section. 
5.2 Magnetic Saturation and Hysteresis loop

The magnetic saturation and the hysteresis loop are the two main problems of the electromagnetic circuit of the magnetorheological fluid brake. Both are magnetic material properties that reflect a magnetic behavior of a magnetic material. Magnetic saturation can be defined as a state of material magnetization after which the apparent viscosity of a magnetorheological fluid is not going to increase no matter what the value of magnetic field intensity is. In other words, no further increasing in the magnetic field density when the magnetic field intensity increase [137, 138]. Magnetic saturation can be described as a function of yield stress for a typical magnetorheological fluid instead of magnetic flux density (B) as shown in Fig. 5-1. Yield stress increases with magnetic field strength $(\mathrm{H})$ until a point that yield stress shows no significant increasing as magnetic field strength increase [139, 140]. Physically speaking, at the saturation state the braking torque is no longer increase as the filed strength increase and simply means any increasing in the input current beyond the saturation current will transform into heat energy which might affect the braking torque rather than increasing it.

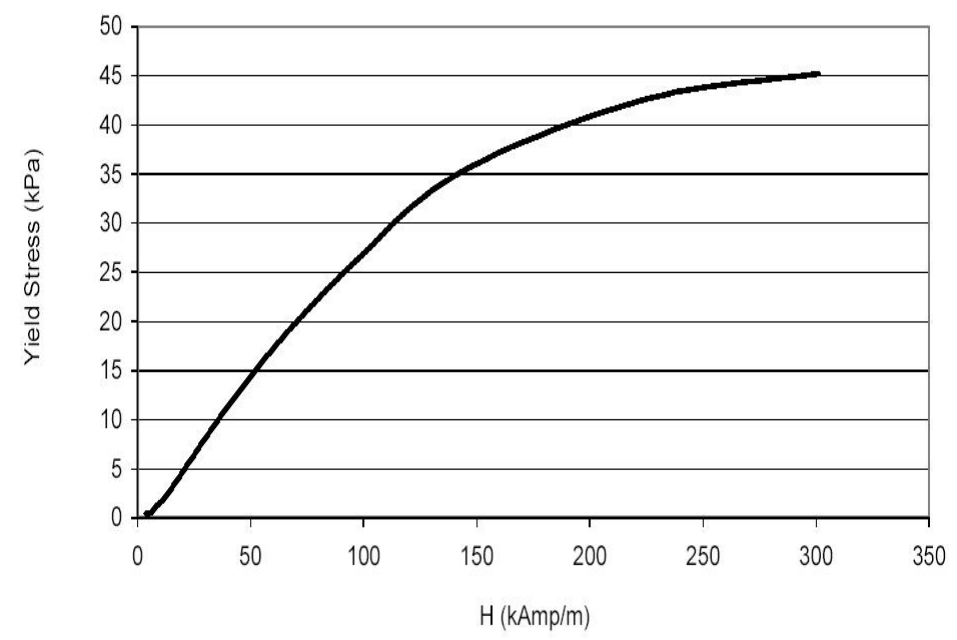

Figure 5-1. Saturated yield stress of a magnetorheological fluid brake [140] 
The second important characteristic (limitation) of the electromagnetic circuit is the hysteresis loop. A hysteresis loop is a plot shows the relation between magnetic flux density and magnetic field strength. This curve (called B-H curve) gives a wide range of information about the magnetic behavior of the magnetic material and some important magnetic properties like material remanence, coercivity, and magnetic reluctance. A typical hysteresis loop of a magnetorheological fluid is given in Fig. 5$2[141]$

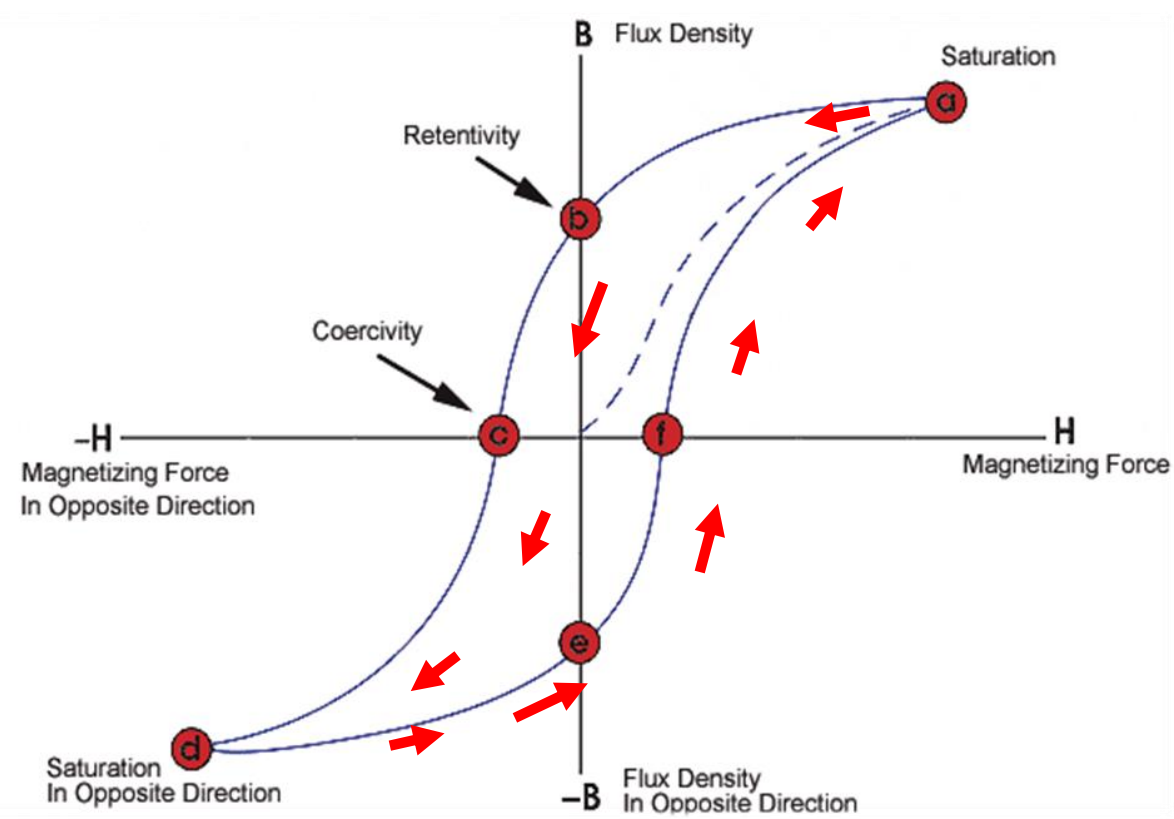

Figure 5-2. A hysteresis loop for a typical magnetic material, adapted from [141]

For the magnetic material being magnetized of the first time, the plot will start from the zero value of the magnetic field strength and follow the dashed line. Magnetic field strength increases with the magnetic field intensity until point a (red circle) to the upper right corner of the loop. The point a (red circle) called the saturation point is mentioned above. The dashed magnetic behavior is not reversible as the magnetic field decreases (or unloads), so it goes to point b (red circle) instead 
of returning to the original point. The main reason for the hysteresis is a residual magnetic field density. The residual magnetic field called magnetic remanence (magnetic effect remains in the magnetic circuit after unloading) or magnetic retentivity. The magnetic retentivity is a material's ability to keep a specific value of magnetic field after the unloading case. This property is a critical factor for magnetorheological fluid brake design and applications. For example, the residual magnetic field keeps the undesired braking torque for magnetorheological fluid brake applications. That means another energy source needs to be applied to completely unload the brake (off-state braking torque).

With further decreasing of the magnetic field, the magnetic saturation occurs in the negative side of the loop, point $d$ (red circle). The point of intersection of the loop within the negative axis of the magnetic field called coercivity. Coercivity is the magnetic field that must be applied to get zero magnetic flux. In other words, coercive force is the force needed to remove or reduce the effect of the residual magnetic field inside the magnetic circuit. In addition, the hysteresis loop gives an indication about how easy it is for the material to become magnetized. A narrow loop shows that the material is easily magnetized while the wider loop refers to magnetic material that is more difficult to magnetize [142].

Finally, magnetic saturation, residual magnetic field, and the hysteresis loop are drawbacks of magnetic circuit designs which limit our ability to obtain an optimal design. We need to find another way to get through a wider space of working and little limitations. In this chapter the brake design will be changed by altering one geometrical design parameter only. In the next section, two new suggested designs based on changing one geometrical design parameter (magnetorheological fluid thickness $(h))$ are presented. 


\subsection{Continuous Variable Fluid Thickness (Torque) MRF Brake-New Suggested Designs (CVFT brake)}

\subsubsection{General}

As it has seen from the literature presented in Chapter 2 the researchers have tried several suggested ways to increase magnetorheological fluid brake torque. Some of them have performed complicated studies to obtain an optimal design of the electromagnetic circuit that could achieve the required magnetic field intensity. However, the problem of magnetic field saturation and hysteresis behavior stopped them to get higher braking torque. Other researchers suggested increasing input current to increase magnetic flux density, but a problem of energy consumption and energy transformation to heat energy was introduced as well. Other researchers suggested changing brake geometry by either increasing disk radius or the surface area of contact by increasing disks number. However, problems of large volume and the viscous torque in an off-field state (in the normal working conditions) are presented. That action adds more energy to overcome high value of viscous torque which is not preferable in the normal working conditions.

For more convenient, several designs and their drawbacks are illustrated in Table (5-1). These drawbacks have motivated us to create a dynamic brake in order to increase braking torque capacity and keep both supplied energy, brake volume, and brake mass as small as possible. The dynamic brake simply means that the braking torque can be increased or decreased not only by the combined effect of fluid viscosity and the applied magnetic field, but also by reducing magnetorheological fluid thickness with time to achieve the required braking torque. In this way, brake volume and the supplied energy can be controlled to be low as possible. The new suggested design is presented in the next sections. 
Table 5-1. Drawbacks of several magnetorheological brake designs

\begin{tabular}{|l|l|}
\hline \multicolumn{1}{|c|}{ Design } & \multicolumn{1}{c|}{ Drawbacks } \\
Single disk magneto- & 1- Low performance (low output torque, \\
2- Multiple disks magneto- & 1- Big size. \\
rheological fluid brake. & 2- Limited applications. \\
& 3- High power consumptions. \\
& 4- High off-field torque (unpreferable). \\
\hline 3- Working on the & 1- Increasing coil windings and power \\
electromagnetic design, coil & consumption. \\
design and configuration. & 2- Massive brake and limited applications. \\
\hline 4- Complex optimization & 3- Magnetic saturation and hysteresis problem. \\
methods (Ansys, Sequential & 2- Keep a designer out of play. \\
\hline
\end{tabular}

It worthy to mention that the new suggested design was not randomly chosen. However, the idea of the new designs is lunched after getting the mathematical modeling of the magnetorheological fluid brake, principle of work, getting the braking torque equation in nondimensional form (to make the study more general), specifying the nondimensional design parameters, and finally studying effect of the design parameters of the braking torque by conducting a sensitivity analysis using Taylor series concept as shown in Chapter 3. The general procedure for developing the study and getting the main idea of developing the new design is given as follows:

1- Study literatures about magnetorheological fluid brake, its design and ways for getting an optimal configuration.

2- Find the important design parameters for magnetorheological fluid brake. 
3- Develop the mathematical modeling taking all the design parameters into accounts and calculate the braking torque due to viscous and magnetic field.

4- Nondimensionalize the important equations like braking torque to make the brake design more general.

5- Conduct the sensitivity analysis using Taylor series concept to understand how the design parameters affect braking torque and specify which of these parameters has/have the predominant impact.

Taking the benefit of sensitivity analyses results and then start to develop the new suggested design. From the first sight on the sensitivity results, it has seen that magnetorheological fluid thickness plays an important role in the braking torque. Since the braking torque is inversely proportional to the fluid thickness as shown in the torque equation in Eq. (3.31) of Chapter 3 then any reduction in the fluid thickness $h$ will increase the braking torque. Taking the advantage of that, new designs considering varying one or two sides of the magnetorheological fluid thickness are created and presented in the next section, respectively

5.3.2 Continuous Variable Fluid Thickness (Torque) of One Variable Fluid Thickness (CVFT1h)

The main key for the new designs of variable fluid thickness is to reduce the fluid thickness using a linear actuator. Figure 5-3 presents the new design of continuous variable torque of one variable fluid thickness (variable fluid thickness on the left side of the rotating-translating disk, $\left.h_{v a r}\right)$. The new design mainly consists of the basic parts of the typical magnetorheological fluid brake. However, the rotating disk is changed to be two separate disks, the first disk rotates only as shown 


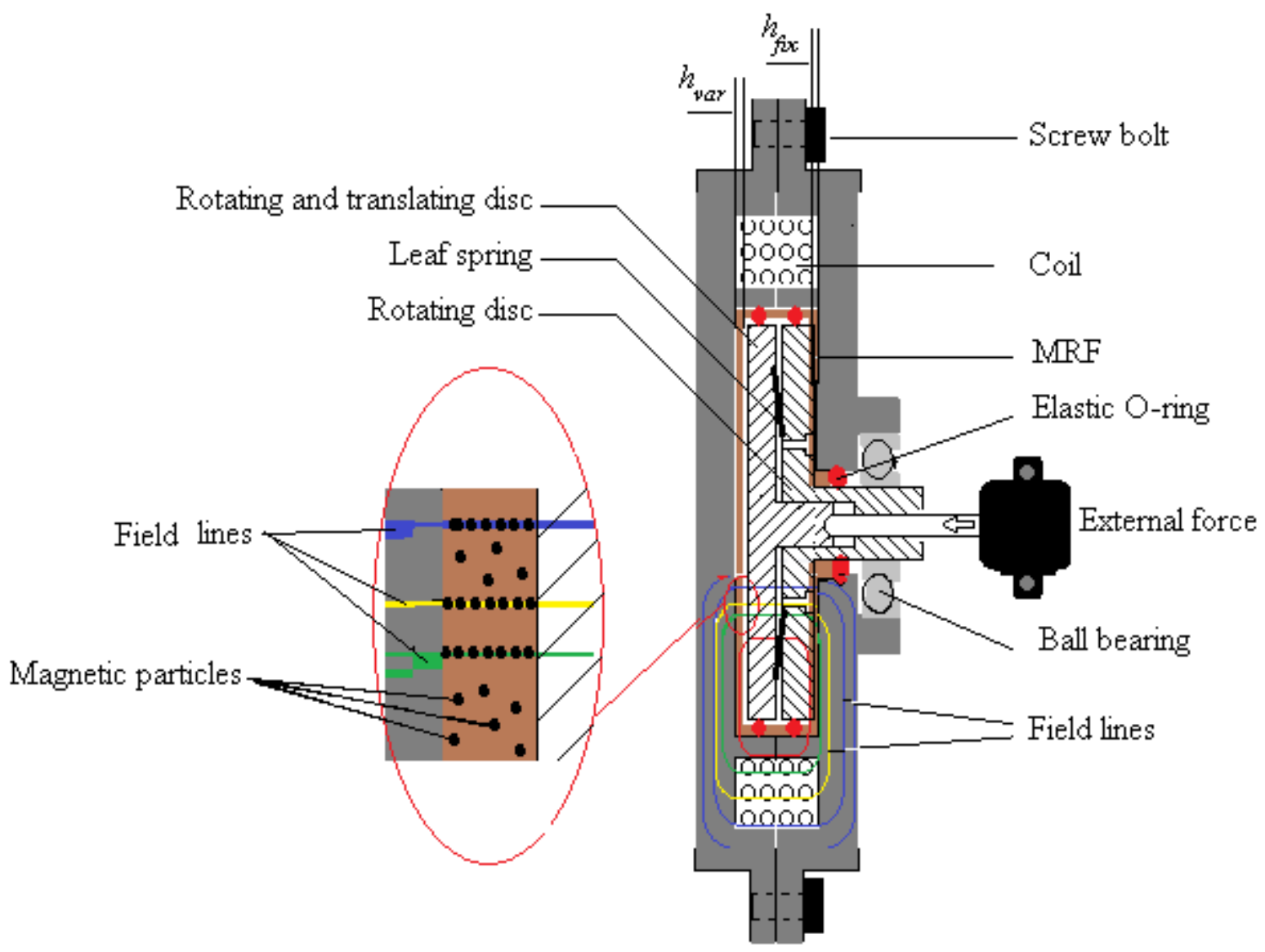

Figure 5-3. A schematic diagram of the suggested design of the dynamic MRF brake (CVFT1h)

In Fig. 5-4a while the second disk rotates and translates simultaneously as shown in Fig. 5-4b. A translational actuator is added to provide an external force which tries to move the rotating-translating disk to the left and reduce the fluid thickness, thus increasing the braking torque. Four leaf springs are attached to the rotating and the rotating-translating disk in order to provide a primary force to keep the two disks in contact when the actuator is in the idle position as shown in Fig. 5-4. In order to achieve an easy translational motion of the second disk and to prevent the relative rotational motion between the two disks, a hexagonal cross section for the inner and outer contact surfaces of the disks is designed. 

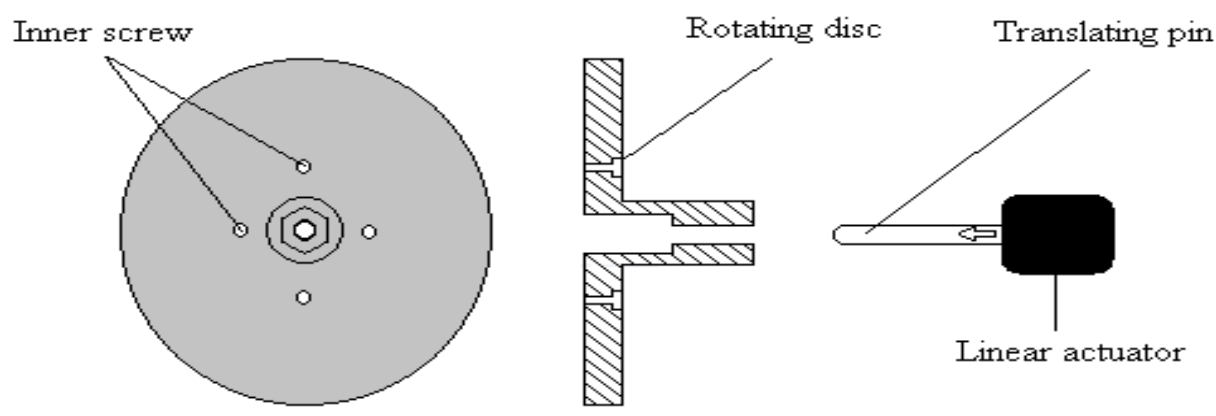

(a) Rotating disk.

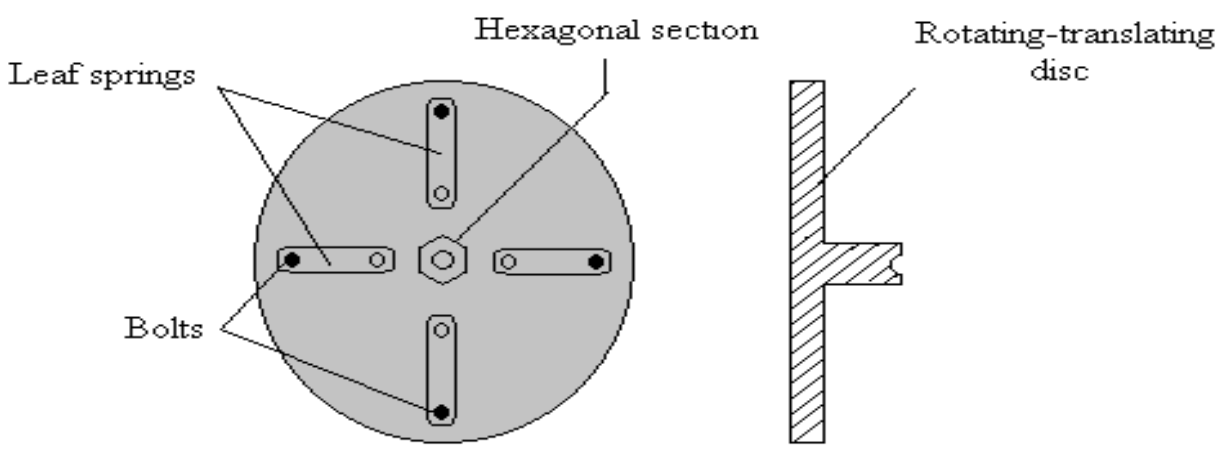

(b) Rotating-translating disk.

Figure 5-4. Rotaintg and rotaing-translating disks design

Figure 5-5 presents the steps of changing the fluid thickness. The actuator produces a limited translational motion (as referred by an arrow headed to the left) and has an external hemispherical end to reduce the frictional force with an internal hemispherical end created at the center of the rotating-translating disk. Pin motion produces the translational motion of the rotating-translating disk which then decreases the fluid thickness. The variations of the fluid thicknees can be controlled by the actuator motion and that can be done by controlling the input current or the pressure drop for an electrical and a neumatic actuator, respectively. 


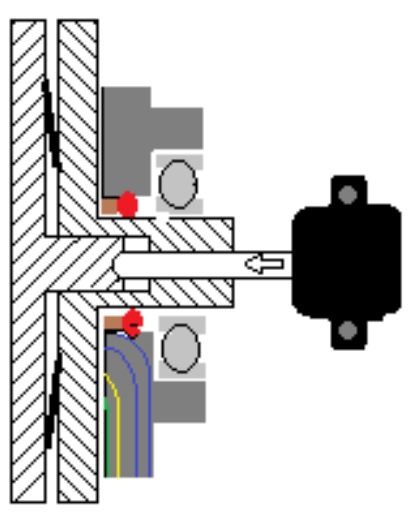

C

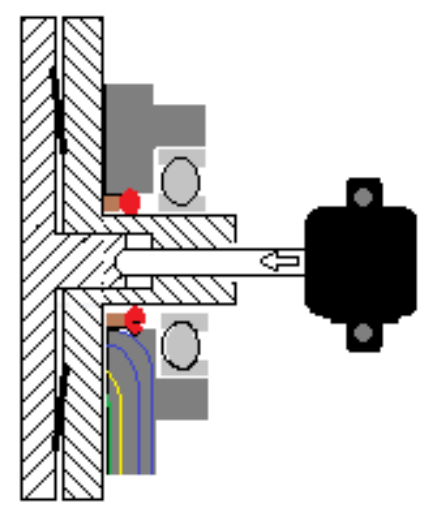

b

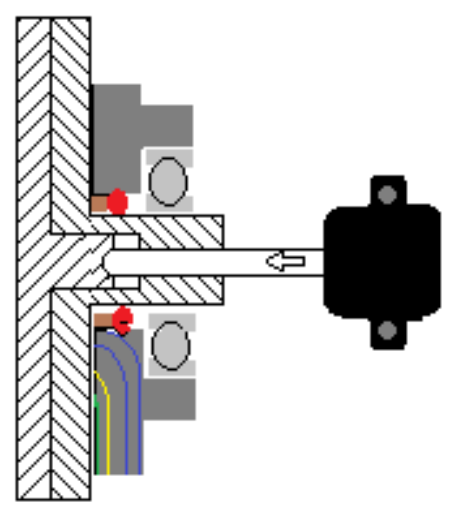

a

Figure 5-5. Steps of changing fluid thickness by the actuator movement

\subsubsection{Updated Mathematical Model for the CVFT1h}

Referring to the torque equation derived in Eq. (3.29) of Chapter 3, the total torque for the typical magnetorheological fluid brake can be given as,

$$
T=\frac{2 \pi N k H^{\beta}}{3}\left(r_{o}^{3}-r_{i}^{3}\right)+\frac{\pi N \omega \mu_{p}}{2 h}\left(r_{o}^{4}-r_{i}^{4}\right) .
$$

For the above equation, the fluid thickness $h$ is constant. However, for the new design of the continuous variable fluid thickness CVFT1 $h$, the magnetorheological fluid thickness is variable and the braking torque in an integral form can be given as follows,

$$
T=2 \pi \int_{r_{i}}^{r_{o}}\left\{\left(N k H^{\beta} \operatorname{sign}(\dot{\gamma})+\mu_{p} \frac{r \omega}{h_{f i x}}\right)+\left(N k H^{\beta} \operatorname{sign}(\dot{\gamma})+\mu_{p} \frac{r \omega}{h_{\text {var }}}\right)\right\} r^{2} d r
$$

where $h_{f i x}$ and $h_{\text {var }}$ are fluid thickness on the rotating and rotating-translating disks, respectively. The sub index (fix) means that the fluid thickness on the rotating disk side stays fixed and independent on the actuator motion while the sub index (var) means that the magnetorheological fluid thickness on the rotating-translating disk side is a variable and varies with actuator motion. It is assumed that variations of the 
magnetic properties are very small and can be neglected. Equation (5.2) can be given as follows:

$$
T=2 \pi \int_{r_{i}}^{r_{o}}\left\{2 N k H^{\beta} \operatorname{sign}(\dot{\gamma})+\mu_{p} r w\left(\frac{1}{h_{f i x}}+\frac{1}{h_{\mathrm{var}}}\right)\right\} r^{2} d r
$$

Magnetic field can be assumed to be constant. For two surfaces of friction (N=2), Eq. (5.3) can be given as follow:

$$
T=\frac{4 \pi k H^{\beta}}{3}\left(r_{o}^{3}-r_{i}^{3}\right)+\frac{\pi \mu_{p} \omega}{2}\left(r_{o}^{4}-r_{i}^{4}\right)\left(\frac{h_{f i x}+h_{\mathrm{var}}}{h_{f i x} h_{\mathrm{var}}}\right) .
$$

\subsubsection{Continuous Variable Fluid Thickness (Torque) of Two Variable Fluid Thicknesses (CVFT2h)}

The same principle of the first design (CVFT1 $h)$ is used for the continuous variable torque of two variable thicknesses (CVFT2 $h$ ). However, the fluid thickness on both sides of the magnetorheological fluid brake are varied (decreased) instead of one side of the fluid thickness for the CVFT1 $h$ design. Figure 5-6 presents the new design of the continuous variable torque of two variable fluid thicknesses CVFT $2 h$ (variable fluid thickness on both sides of the rotating-translating disks, $h_{v a r}$ ). It mainly consists of the basic parts of the typical magnetorheological fluid brake and the CVFT1 $h$ design, but two rotating-translating disks on both sides of magnetorheological fluid brake are designed instead of one disk in CVFT1 $h$. They are rotating-translating $(\mathrm{L})$ and rotating-translating $(\mathrm{R})$ on the left and right sides, respectively as shown in Fig. 5-7. The rotating-translating disks are freely to move to the left and right directions, respectively. Two ball bearings are attached to the main body of the brake to support the rotating shaft and to ensure the concentricity during the loading case. The rotating shaft is designed to have four grooves (four 
rectangular passes) as guides for the pushing part as shown in Fig. 5-7 (spider plate) to move freely along these passes.

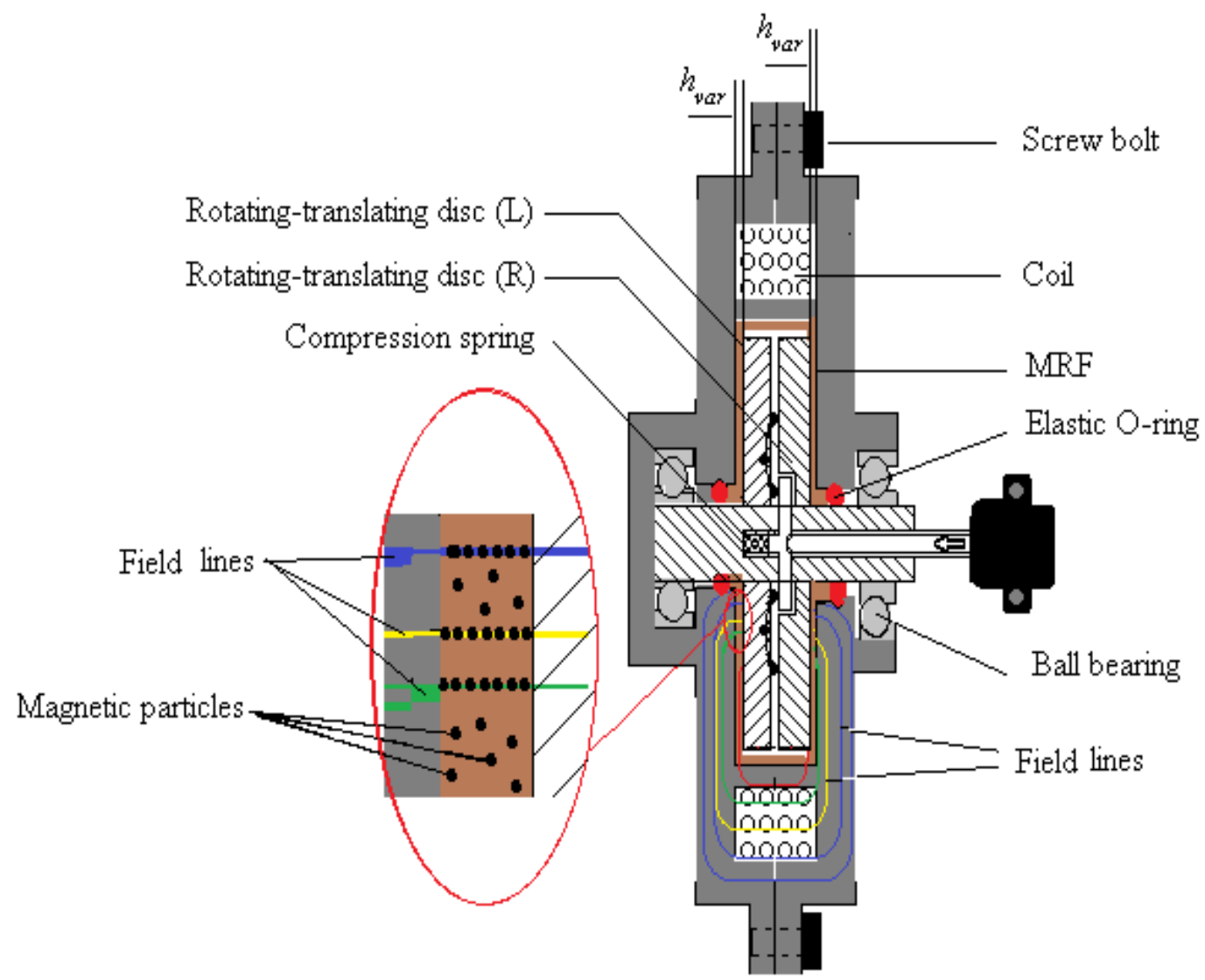

Figure. 5-6. A schematic diagram of the suggested design of the dynamic MRF brake $(\mathrm{CVFT} 2 h)$

The main function of the pushing part (spider plate) is to transmit the applied force by the actuator to another part, action-reaction ring as shown in Fig. 5-7. Four action-reaction rings are designed to be attached to the rotating-translating disk on the left side (L) at its centers using a small pin to make it easy to rotate freely about the pin. The two ends of these rings are designed to get hemispherical ends to reduce the frictional forces. A small compression spring is attached as shown in Fig. 5-7 to provide a preliminary compressive force acting on the spider plate (pushing part) to keep it in contact with rotating-translating disk $(\mathrm{R})$ in the idle working condition. The 
reason for this is to deactivate the brake function in the normal working condition first and to reduce the noise that might be resulted from the uncontrolled movement of the spider part during the repeated activation and deactivation processes of the actuator second.

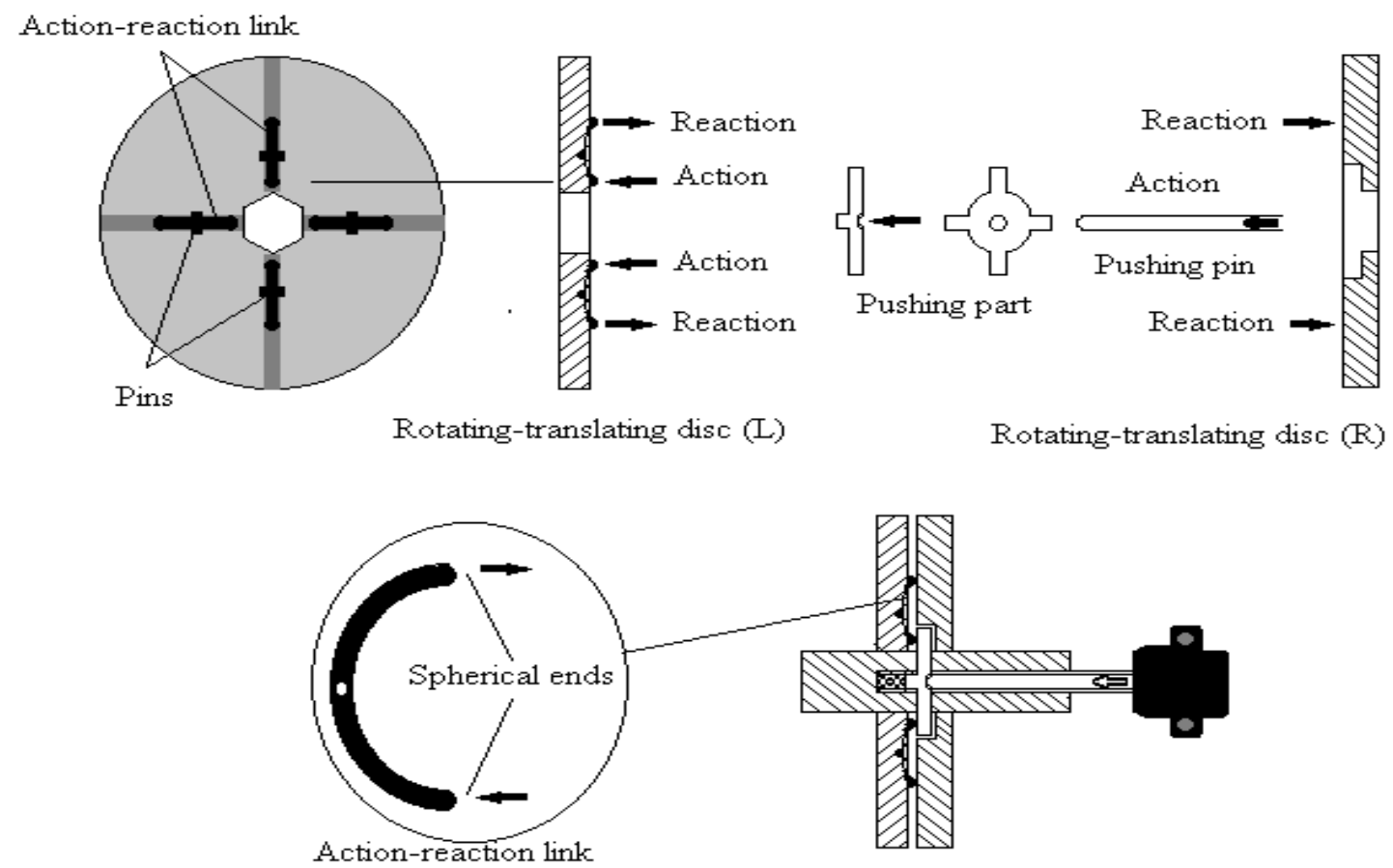

Figure 5-7. Components of the two variable fluid thicknesses brake (CVFT2h)

To achieve an easy translational motion of the two disks on the main shaft, a hexagonal cross section for the inner contact surface of the disks is designed to prevent the relative rotational motion of the disks and keep them rotate at the same rotational velocity. The working principle of this brake is very simple. The actuator produces a limited translational motion (as referred by an arrow headed to the left). The actuator pin has an external hemispherical end to reduce the frictional force with an internal hemispherical end created at the center of the pushing plate (spider plate). The spider plate pushes the four action-reaction rings. Two reactions forces are built 
up, the first one is located at the center of each action-reaction ring and try to move the left rotating-translating disk to the left. The second reaction force (as shown in Fig. 5-7) try to move the right rotating-translating disk to the right. The variations of the fluid thicknees can be controlled by the actuator motion and that can be done by controlling the input current or the pressure drop for an electrical a neumatic actuator, respectively.

\subsubsection{Updated Mathematical Model for the CVFT2h}

Referring to the torque equation derived in for the CVFT1 $h$, Eq. (5.2) can be given as follows,

$$
T=2 \pi \int_{r_{i}}^{r_{0}}\left\{\left(N k H^{\beta} \operatorname{sign}(\dot{\gamma})+\mu_{p} \frac{r \omega}{h_{\mathrm{var}}}\right)+\left(N k H^{\beta} \operatorname{sign}(\dot{\gamma})+\mu_{p} \frac{r \omega}{h_{\mathrm{var}}}\right)\right\} r^{2} d r
$$

where $h_{\text {var }}$ are the fluid thickness on both sides of the two rotating-translating disks. The sub index (var) means the fluid thickness on both sides of the Disks varies with the actuator motion. It is assumed that variation of the magnetic properties is very small and can be neglected. Equation (5.5) can be given as follows:

$$
T=2 \pi \int_{r_{i}}^{r_{o}}\left\{2 N k H^{\beta} \operatorname{sign}(\dot{\gamma})+\mu_{p} r w\left(\frac{1}{h_{\mathrm{var}}}+\frac{1}{h_{\mathrm{var}}}\right)\right\} r^{2} d r
$$

Magnetic field can be assumed to be constant. For two surfaces of friction ( $N=2)$, Eq. (5.6) can be given as follow:

$$
T=\frac{4 \pi k H^{\beta}}{3}\left(r_{o}^{3}-r_{i}^{3}\right)+\frac{\pi \mu_{p} \omega}{2}\left(r_{o}^{4}-r_{i}^{4}\right)\left(\frac{2}{h_{\mathrm{var}}}\right) .
$$

Eq. (5.7) is a function of $h_{\mathrm{var}}$ only because the fluid thickness varies on both sides of the rotating-translating disks. A simple comparison between torque equation for the old design of the magnetorheological fluid Eq. (5.1) and Eq. (5.7) shows that the 
magnification factor is just doubled ( 2 the numerator) and this is the originality of the new suggested design

\subsubsection{Simple Calculations}

Very simple calculations have been done to explain how magnetorheological fluid thickness variations increases the braking torque. It is assumed that the terms $\left(h_{f i x}+h_{\text {var }} / h_{f i x} h_{\text {var }}\right)$ in Eq. (5.4) and $\left(2 / h_{\text {var }}\right)$ in Eq. (5.7) are braking torque magnification factors for continuous variable fluid thickness CVFT1 $h$ and CVFT2 $h$ designs, respectively. The two factors are simply show how the braking torque is going to be increased due to the thickness variations. Effect of MRF thickness changes $h_{\text {var }}$ can be represented in Fig. 5-8. It is assumed that $h_{f i x}=1 \mathrm{~mm}$ and $h_{\text {var }}$ decreases from $1 \mathrm{~mm}$ to $0.5 \mathrm{~mm}$ only.

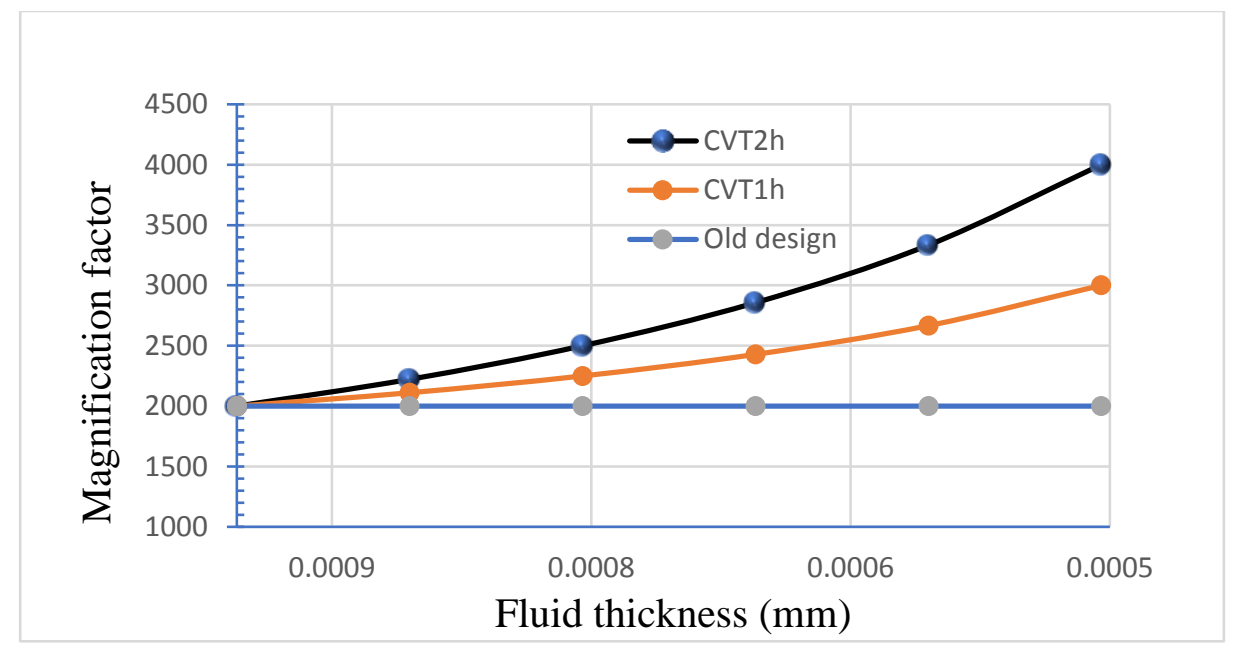

Figure 5-8. Effect of MRF thickness variations on the braking torque

One can easily note that scaling factors are nonlinearly increases as magnetorheological fluid thickness decreases for both CVFT $1 h$ and CVFT2 $h$ designs while the scaling factor of the old design presents no change because the fluid 
thickness is constant on both sides. The scaling factor is increased 50\% (2000 increased to 3000) and a 100\% (2000 increased to 4000) for the CVFT1 $h$ and CVFT2 $h$, respectively. In other words, the braking torque is more sensitive for small variations of the fluid thickness in the CVFT2 $h$ than that corresponding in CVFT1 $h$.

\subsection{Summary}

In this chapter, a general introduction about the optimization methods are introduced. Several designs of magnetorheological fluid brake with its drawbacks are presented. Magnetic saturation and hysteresis problems are discussed to specify the limitations of working of the electromagnetic circuit. Next, the general procedure and reasons beyond developing a new designs of continuous variable magnetorheological fluid brake are illustrated. Next, the two new designs are presented, and principle of work are discussed and explained in detail. Next, the mathematical modelling of these designs starting from the torque equation presented in chapter three is updated. Finally, simple calculations to explain the originality of the new designs are carried out. Controlling the MR fluid brake is presented in the next chapter. 
CHAPTER 6. CONTROLLING A MAGNETORHEOLOGICAL (MR) FLUID

\section{BRAKE}

\subsection{Introduction}

In Chapter 5, we have introduced new suggested designs to increase the braking torque due to change in fluid thickness. One day, these designs are going to serve in the industry because the wide range and versatile applications of the magnetorheological fluid brakes. In the industry, some of working conditions vary with time because of uncertainty and unexpected variations of uncontrolled factors. Controlling process to substitute and control the baking torque due to conditions variation is mandatory. Some applications might require very fast and sensitive response; the controlling will be used to achieve a fast braking response and other response characteristics. Rotating mechanical systems are typically designed to include a brake or a rotational damping device for the purposes of controlling speed. Historically the braking device has been constructed using a friction brake design, which has often been accompanied with adverse wear and premature failure. In order to prevent material wear, and to apply direct electrical control to the brake, magnetorheological (MR) fluid brakes have been introduced for rotational systems.

MR fluid brakes are designed based upon principles that govern viscous dampers where fluid shear-stress is used to resist the motion of mechanical parts. The novel property of MR fluids is that their apparent viscosity can be significantly altered by exposing the fluid to an electrically induced magnetic field. By controlling the strength of the magnetic field, the MR fluid viscosity is easily controlled with a very fast response time and a high torque-to-volume ratio. MR fluid technology holds great promise for many electrically controlled, mechanical systems including brakes and 
clutches.

Within the body of literature known so far, the control for MR fluid brakes has been achieved by altering the input current to the electrical coil. This is the obvious control input; however, an additional option exists for controlling the brake by simultaneously altering both the input current and the MR fluid thickness. This simultaneous control may be used to achieve a wider range of torque without increasing the radial envelope as presented in Chapter 5 for the new MR fluid designs, and to compensate for the saturation limits that occur within electromagnetic fields. This chapter has been written to demonstrate this novel control method for a single disk design, and to show that good dynamic response may be achieved using two proportional-integral (PI) controllers in parallel.

\subsection{Principle of Work}

Solenoid is a kind of the fast actuators used for the hydraulic applications. It mainly consists of two parts, mechanical and electrical. It is simple in design and fast in response. The solenoid will be used as an external actuator which provides the translational motion in CVFT $1 h$ and CVFT2 $h$ brakes. Principle of work and mathematical modeling is presented in the next sections.

\subsubsection{Principle of Work of a Solenoid and the Braking Torque}

The solenoid is an electromechanical actuator consists of two parts, mechanical and electrical parts. It mainly works depending on the electromagnetism theory. Fig. 7-1 shows typical examples of an electromechanical actuators. The main parts of the solenoid are an electrical coil, restoring compression spring, armature, and pushing shaft rigidly connected to the armature. 


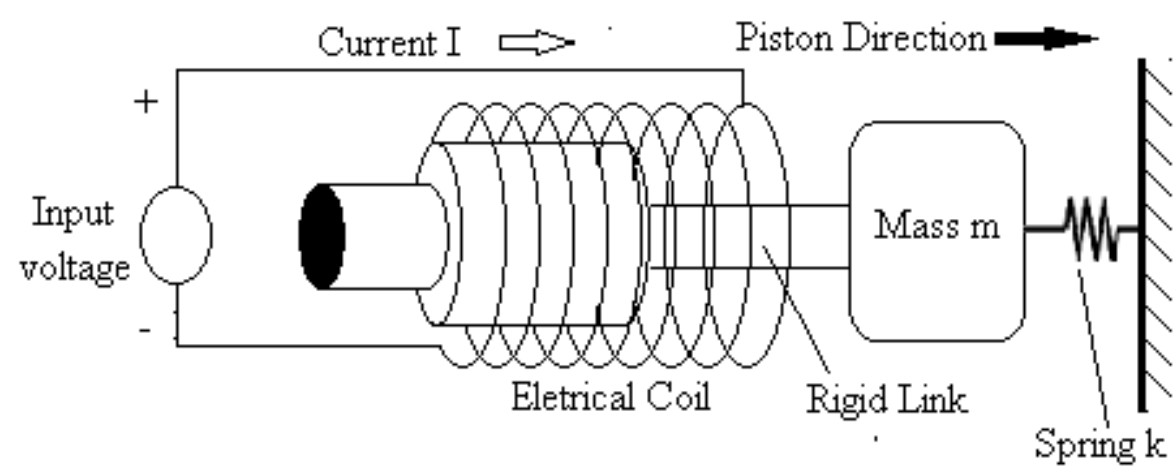

Figure 6-1. A typical electromechanical actuator

The armature moves in translational motion in the horizontal direction. Once an electrical current pass through the coil, an electromagnetic force (attractive force) is produced trying to pull the armature and the pushing shaft into the left direction (considered as the positive direction of the translational motion). After the armature and the rigid pushing shaft got displaced away from the equilibrium position, a restoring force is created by the compression spring which works against the armature motion to displace it back to the original equilibrium point after the applied current is removed.

The armature and the rigid shaft are connected to the pushing shaft of the magnetorheological fluid brake. Therefore, once the armature moves to the left, the rotating-translating shaft/shafts of the brake moves as well in the same magnitude of the armature displacement. In other words, the translational motion of the brake shafts in both CVFTs brakes can be controlled by controlling the translational motion of the armature or the input current of the solenoid.

\subsubsection{Braking Torque}

The mathematical formulation for the new designs of the magnetorheological fluid brake, CVFT $1 h$ and CVFT2 $h$ is presented in detail in Chapter 3 and Chapter 5, 
respectively. For more convenient, the braking torque equations for both designs are given as follows:

$$
T=\frac{4 \pi k H^{\beta}}{3}\left(r_{o}^{3}-r_{i}^{3}\right)+\frac{\pi \mu_{p} \omega}{2}\left(r_{o}^{4}-r_{i}^{4}\right)\left(\frac{h_{f i x}+h_{\mathrm{var}}}{h_{f i x} h_{\mathrm{var}}}\right) .
$$

and

$$
T=\frac{4 \pi k H^{\beta}}{3}\left(r_{o}^{3}-r_{i}^{3}\right)+\frac{\pi \mu_{p} \omega}{2}\left(r_{o}^{4}-r_{i}^{4}\right)\left(\frac{2}{h_{\mathrm{var}}}\right) .
$$

where $h_{f i x}$ and $h_{\text {var }}$ are fluid thickness of the rotating and rotating-translating discs, respectively. One can easily notice that the braking torque equations are functions of $h_{f i x}$ and $h_{\text {var }}$ which is in turn are functions of the actuator translational motion $x$. The magnetorheological fluid thickness variations can be given as $h_{\mathrm{var}}(t)=x(t)$ and $h_{\mathrm{var}}(t)=0.5 x(t)$ for CVFT $1 h$ and CVFT2 $h$, respectively. That simply means controlling the solenoid translational motion is the same as controlling the fluid thickness for the MR fluid brakes.

\subsection{Mathematical Modeling of an electromechanical Solenoid}

In this section, a detailed mathematical modeling of the electromechanical actuator is presented. This section is divided into two sections, mechanical and electrical parts.

\subsubsection{Mechanical Part}

The mechanical part of the actuator can be simplified as a simple spring-mass system with a frictional damping. The mechanical representation of the solenoid is presented in Fig. 6-2. The external force is the electromagnetic force resulted from the current application to the coil. 


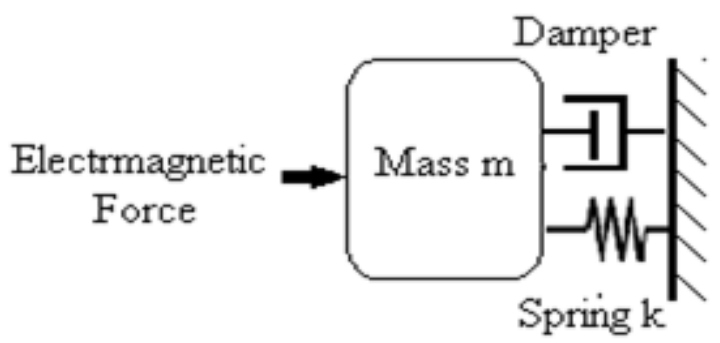

Figure 6-2. Schematic Representation for the Mechanical part of the actuator

\subsubsection{Free Body Diagram}

The free body diagram can be easily determined by choosing the positive direction of the equivalent system mass. The spring is compressed when the equivalent mass moves in the positive direction producing a restoring force of $k x$ where $k$ is the spring constant and $x$ is the displacement in the positive direction. The damping or frictional force is produced in the opposite direction of the mass trying to damp the mass translational movement out. The damping force $b \dot{x}$ depends on mass velocity and $b$ is damper coefficient. Figure 6-3 shows the free body diagram of the equivalent spring-mass system.

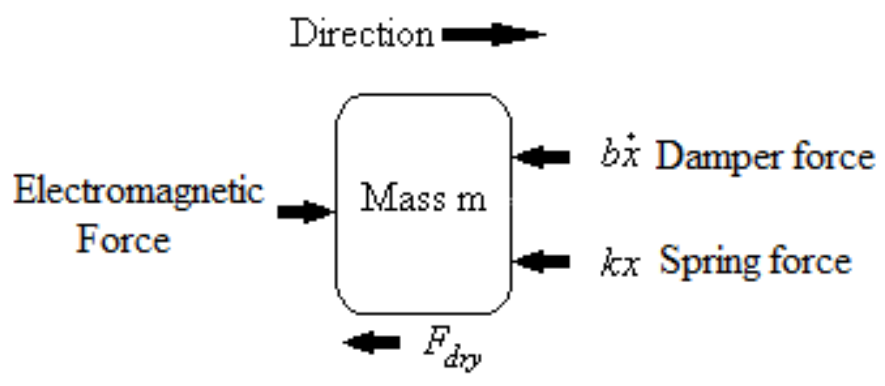

Figure 6-3. Free body diagram of the equivalent spring-mass system of the solenoid.

\subsubsection{Newton's Second Law}

The next step for the mathematical modeling of the mechanical part is to use Newton's second law for translational motion of the equivalent translated mass $m$. 
The summation of all external forces acting on the mass is equal to the inertia force resulted by mass acceleration in a specific direction:

$$
\sum F=m \ddot{x}
$$

By applying Eq. (6.3) and using the free body diagram in Fig. 6-3, the second order governing differential equation of the translated mass can be given as:

$$
\sum F=F_{e m}-k x-b \dot{x}-F_{d r y} \operatorname{sgn}(\dot{x})=m \ddot{x}
$$

which can be simplified into,

$$
m \ddot{x}+b \dot{x}+F_{d r y} \operatorname{sgn}(\dot{x})+k x=F_{e m}
$$

\subsubsection{Electrical part}

The electromagnetic circuit of the actuator is shown in Fig. 6-4. It mainly consists of an external voltage source $e_{i n}$, inductance $L$, and resistance $R$. The magnetic inductance of the solenoid is basically varying nonlinearly with pin displacement.

The magnetic inductance can be given as [158],

$$
L(x)=\frac{L_{o}}{1-x / d}
$$

where $L_{o}$ is the coil inductance in idle solenoid conditions $(x=0)$ which depends on solenoid design parameters so that $L_{o}=\mu_{s} A_{s} N_{t}^{2} / l_{s}$ where $\mu_{s}$ is the magnetic permeabilities of the air and solenoid core, $A_{s}$ is the cross sectional area of the air gap of the solenoid, $N_{t}$ is the number of turns of the solenoid coil, $l_{s}$ is the length of the coil.

\section{Kirchhoff's Law}

The summation of all external voltage applied to an electric circuit as shown 


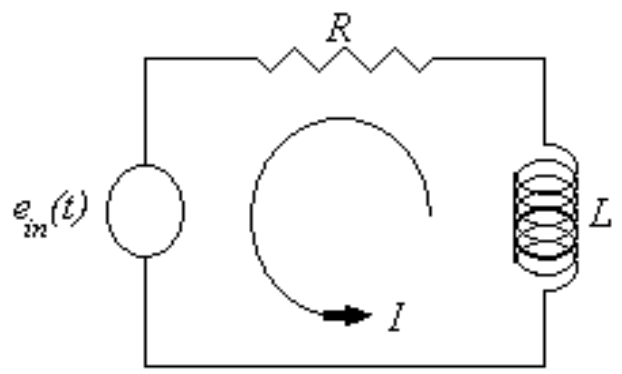

Fig. 6-4 A schematic diagram of the electric part of the actuator

in Fig. 6-4 must be equivalent to summation of all the internal component voltage as shown:

$$
e_{\text {in }}(t)-e_{R}-e_{L}=0
$$

where $e_{R}$ and $e_{L}$ are the resistance and inductance voltages, respectively. The inductance voltage is defined as the variation of the corresponding magnetic flux induced through the solenoid coil with time as $e_{L}=\dot{\lambda}$ where $\lambda$ is the magnetic flux which can be assumed as $L(x) I_{s}$ where $I_{s}$ is the solenoid current. Since the inductance varies with the displacement first and with time second, one can use the chain rule to obtain the inductance voltage as,

$$
\dot{\lambda}=\frac{d L(x)}{d x} \dot{x}+L(x) \dot{I}
$$

where $\dot{x}$ and $\dot{I}$ is solenoid velocity and time derivative of the current, respectively. The resistance voltage can be given by $e_{R}=R I_{s}$. Using Eq. (6.8) in Eq. (6.7), the solenoid electrical model can be given as,

$$
L(x) \dot{I}+R I=e_{i n}(t)-\frac{d L(x)}{d x} I \dot{x} .
$$

For this solenoid, it can be assumed that coil inductance is constant and considering the voltages across the inductance and the resistance [158]: 


$$
L \dot{I}+R I=e_{i n}(t)-K I \dot{x}
$$

where $K=d L(x) / d x$. Back to Eq. (6.5) the electromagnetic force $F_{e m}$ is a function of the stored energy inside the coil of the solenoid. It is known that the stored energy through the coil is a function of the coil current and inductance as $E_{s}=\frac{1}{2} L(x) I^{2}$ and therefore, the electromagnetic force is the energy derivative to the displacement $x$ which can be given by,

$$
F_{e m}=\frac{1}{2} \frac{d L(x)}{d x} I^{2}
$$

Three state variables can be developed for the second order and first order differential equations for mechanical and electrical parts of solenoid, respectively. The state variables are $x, \dot{x}$, and $I$

$$
\begin{aligned}
L \dot{I}+R I & =e_{i n}(t)-K I \dot{x} \\
F_{e m} & =0.5 K I^{2}
\end{aligned}
$$

\subsection{MR Fluid Brake Control}

In this section, the first example of controlling a MR fluid brake by simultaneously altering both the input current to the electric coil, and the MR fluid thickness between the stationary and rotating part of a single-disk brake is presented. In the development of the nonlinear torque-model for the brake, nondimensional analysis will be used to generalize the problem for any brake configuration with similar attributes, while providing design guidance for making the energized (onfield) and non-energized (off-field) brake components comparable in strength. In order to control the brake speed, two saturating proportional-integral (PI) controllers are used in parallel: one for adjusting the MR fluid thickness, and one for adjusting the input current to the electric coil. 


\subsubsection{Descriptions}

A schematic for a single disk, MR fluid brake is shown in Fig. 6-5. The lefthand-side of this figure shows a non-energized rotating disk connected to a shaft. For more convenient, the angular velocity of the disk is given by $\omega$ and the torque generated by the brake is shown by the symbol $T$. Magneto-rheological (MR) fluid is shown to exist between the rotating disk and a stationary surface.

The thickness of the MR fluid is given by the dimension $h$. As it turns out, in the absence of a magnetic field, MR fluid sustains a shear stress that is proportional to its strain rate which means that the non-energized (off-field) MR fluid is Newtonian and characterized by a certain fluid viscosity. In this non-energized state, the shear stress in the MR fluid generates a brake torque that is proportional to the fluid viscosity and the angular velocity, while being inversely proportional to the MR fluid thickness $h$.The right-hand-side of Fig. 6-5 shows the same brake geometry but with a magnetic field that has been generated by passing current through the electric coil which is shown to be wrapped around the brake. The current is depicted in Fig. 6-5 using the symbol $i$ which flows through the coil into the paper at the top, and out of the paper at the bottom. The novel property of a MR fluid is that the viscosity of the fluid is increased when exposed to a magnetic field. This additional viscosity of the fluid increases with increased magnetic-flux density which is proportional to the current passing through the electric coil. In other words, increasing the current through the coil increases the viscosity of the MR fluid, which then increases the resistive torque generated by the brake.

A closed-loop control for the MR fluid brake is designed by simultaneously adjusting the MR fluid thickness $h$ and the coil current $i$. The actuator for adjusting the fluid thickness and the driver for adjusting the coil current are not depicted in 
Fig. 6-5, and will be idealized for illustrating the control method.

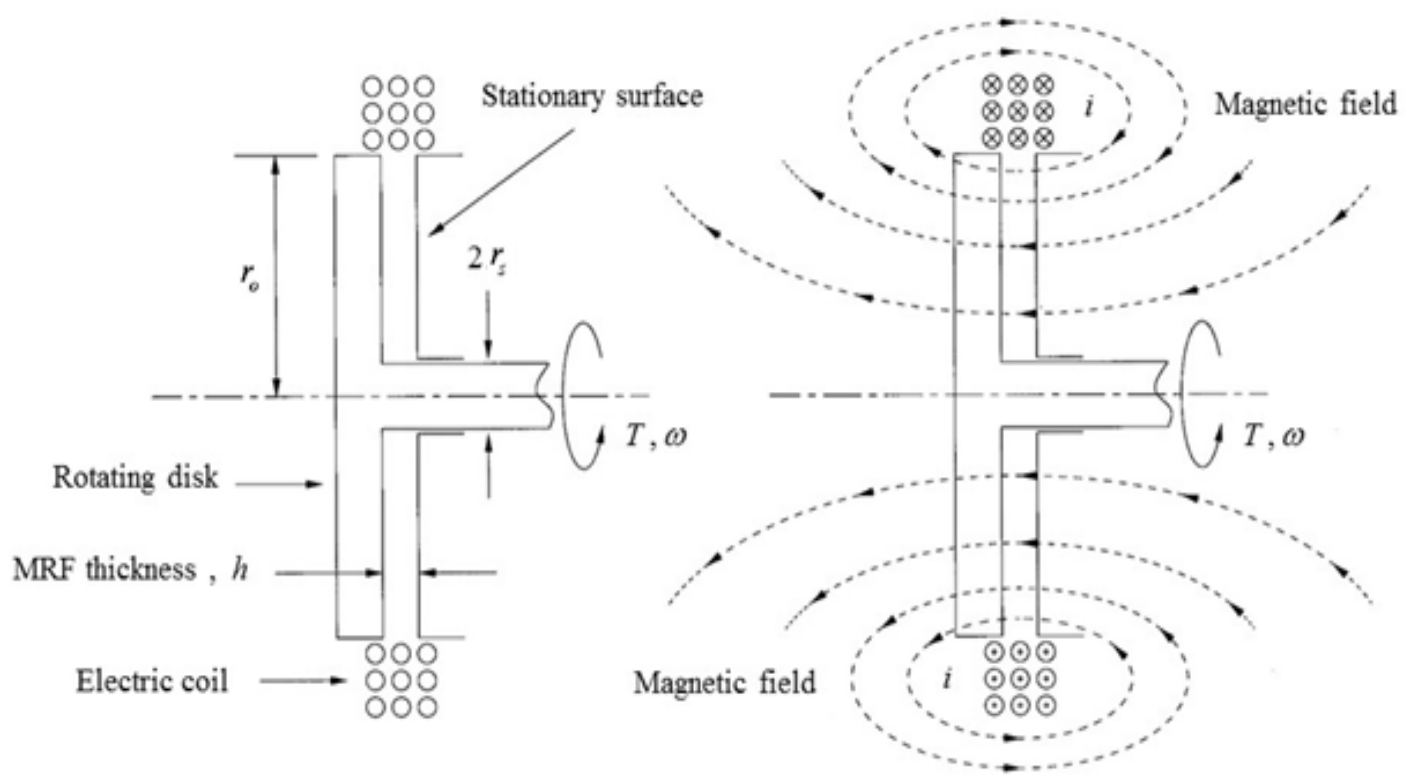

Figure 6-5. Schematic of the single disk, MR fluid brake

Note: the linear actuator for adjusting the fluid thickness will be fairly small (no additional radial space required) due to the fact that the force requirements will be low. There are three reasons for designing a controller that adjusts both $h$ and $i$ as opposed to making only one adjustment. First, an adjustment for the fluid thickness cannot be used to fully stop the brake, and therefore a control for the electric current is necessary if zero velocity is desired. Second, the fluid thickness and the electric current are both limited in their range of operation and when one parameter reaches a saturation point the other parameter continues to regulate the brake speed. Third, by adjusting both parameters a wider range of braking torque may be achieved. For most operating conditions, the controller will be simultaneously adjusting both $h$ and $i$; however, a simplified way to think about this control is to consider the adjustments for $h$ to be applied primarily for controlling high angular-velocities while 
adjustments for $i$ are applied primarily for controlling low angular-velocities. This control design will be clarified in the sections that follow.

\subsubsection{Analysis}

\subsubsection{Dynamic System}

Figure 6-6 shows a schematic of the rotating system that is to be controlled by the MR fluid brake. In this schematic, the left-hand-side represents the MR fluid brake which exerts a control torque $T$ on the rotating flywheel. The flywheel is shown to have a mass moment-of-inertia given by $J$. On the right-hand-side of this schematic, the input torque to the rotating system is given by the load torque $T_{L}$. The system rotates about the central axis with an instantaneous angular-velocity $\omega$.

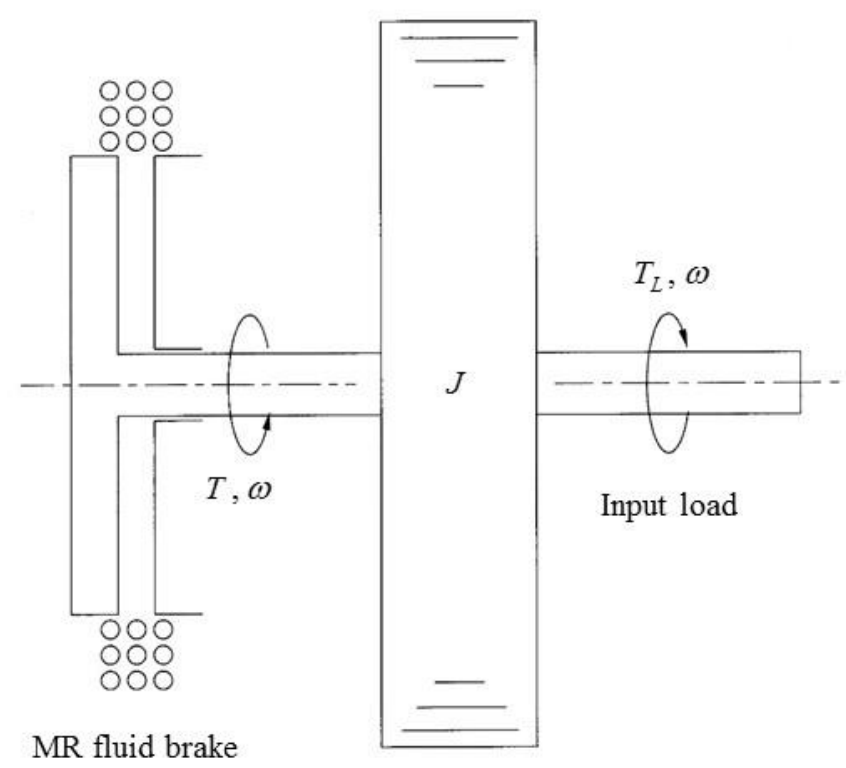

Figure 6-6. Schematic of the rotating system being controlled by the MR fluid brake

According to Newton's second law, the equation of motion for the rotating system shown in Fig. 6-6 is given by 


$$
J \frac{d \omega}{d t}=T_{L}-T
$$

where $t$ is time. In a subsequent section of this chapter, a feedback control system will be designed for regulating the rotational speed $\omega$ by altering the brake torque itself. The braking torque is derived in Ch.3 (Eq. (3.31)). The only different in this section is to refer the shear stress due to magnetic field by a magnetic- flux density for simplicity purpose so that $\tau_{B}=k B^{\alpha}$ where $B$ is the magnetic-flux density generated by the electric coil, and $k$ and $\alpha$ are material properties for a specific MR fluid. As it turns out $\alpha$ is near unity for many MR fluids [37,155] and the magnetic-flux density is proportional to the electric current for most operating conditions of the coil [156]. If we use a MR fluid with $\alpha=1$, and if we stay within the linear range of the magnetic-flux density as it varies with current, then the induced shear-stress resulting from the magnetic field may be written as

$$
k B^{\alpha}=\frac{\tau_{B}}{i_{\max }} i
$$

where $\tau_{B}$ is the maximum shear yield-stress that may be added to the MR fluid when the maximum allowable current $i_{\max }$ is applied to the coil. Estimates for $\tau_{B}$ are readily obtained from MR fluid suppliers [157]. Using Eq. (6.14), the total torque that is generated by the MR fluid brake in terms of magnetic-flux can be written as

$$
T=\frac{\pi}{2} \frac{\mu \omega}{h} r_{o}^{4}\left[1-\left(\frac{r_{s}}{r_{o}}\right)^{4}\right]+\frac{2 \pi}{3} \tau_{B} \frac{i}{i_{\max }} r_{o}^{3}\left[1-\left(\frac{r_{s}}{r_{o}}\right)^{3}\right] .
$$

The first term on the right-hand-side of Eq. (6.15) represents the resistance applied by the non-energized (off-field) brake while the second term represents the additional resistance that is applied by exposing the MR fluid to the magnetic field produced by the electromagnetic circuit of the MRF brake. 


\subsubsection{Nondimensional Analysis}

It is convenient to nondimensionalize the governing equations by using the following definitions:

$$
\begin{gathered}
h=h_{o} \hat{h} \quad, \quad i=i_{\max } \hat{i} \quad, \quad r_{s}=r_{o} \hat{r}_{s}, \\
T=T_{o} \hat{T} \quad, \quad T_{L}=T_{o} \hat{T}_{L}, \quad t=t_{o} \hat{t} \text { and } \omega=\omega_{o} \hat{\omega}
\end{gathered}
$$

where $h_{o}$ is the nominal MR fluid thickness, $T_{o}$ is the nominal load torque, $\omega_{o}$ is the nominal angular velocity, $t_{o}$ is a characteristic time constant for the dynamic response, and all other parameters have been previously defined. In Eq. (6.16) the symbols with carets are dimensionless and of order one. Substituting Eq.s (6.16) and (6.15) into Eq. (6.13) produces the following nondimensional equation of motion for the rotating system shown in Fig. 6-6:

$$
\frac{d \hat{\omega}}{d \hat{t}}=\hat{T}_{L}-\frac{\hat{\omega}}{\hat{h}}-\hat{i}
$$

In order to produce this equation, the following definitions were used for the nominal load torque and the characteristic time:

$$
T_{o}=\frac{\pi}{2} \frac{\mu \omega_{o}}{h_{o}} r_{o}^{4}\left(1-\hat{r}_{s}^{4}\right) \text { and } t_{o}=\frac{J \omega_{o}}{T_{o}}
$$

In addition to using these definitions, the following design constraint was enforced:

$$
\frac{4}{3} \frac{\tau_{B}}{\mu \omega_{o}} \frac{h_{o}}{r_{o}} \frac{1-\hat{r}_{s}^{3}}{1-\hat{r}_{s}^{4}}=1
$$

Physically speaking, this design constraint ensures that the non-energized braking torque and the magnetic-field braking torque are similar in magnitude when applied. As shown in the Appendix, this design constraint is easily satisfied using realistic numbers.

In looking at Eq. (6.17) the reader should be impressed with the elegance and simplicity of the governing equation. In this nondimensional form, which captures all 
of the physics of the problem, the rotational speed of the system depends upon only three things: 1) the nondimensional load torque $\hat{T}_{L}, 2$ ) the nondimensional MR fluid thickness $\hat{h}$, and 3) the nondimensional current $\hat{i}$. Furthermore, all of these parameters are of order one for reasonable brake operation. In the section that follows, a control law will be presented for simultaneously adjusting $\hat{h}$ and $\hat{i}$ in order to regulate the angular velocity $\hat{\omega}$.

\subsubsection{Control Design}

\subsubsection{General}

The objective of our controller is to regulate the speed of the rotational system shown in Fig. 6- 6 by altering the MR fluid brake torque $\hat{T}$, which is adjusted by simultaneously altering the MR fluid thickness $\hat{h}$ and the electric coil current $\hat{i}$. A block diagram for this controller is shown in Fig. 6-7 with two saturating PI controllers and another nonlinear block which describes the inverse proportionality of the MR fluid thickness for the non-energized torque that is developed by the brake.

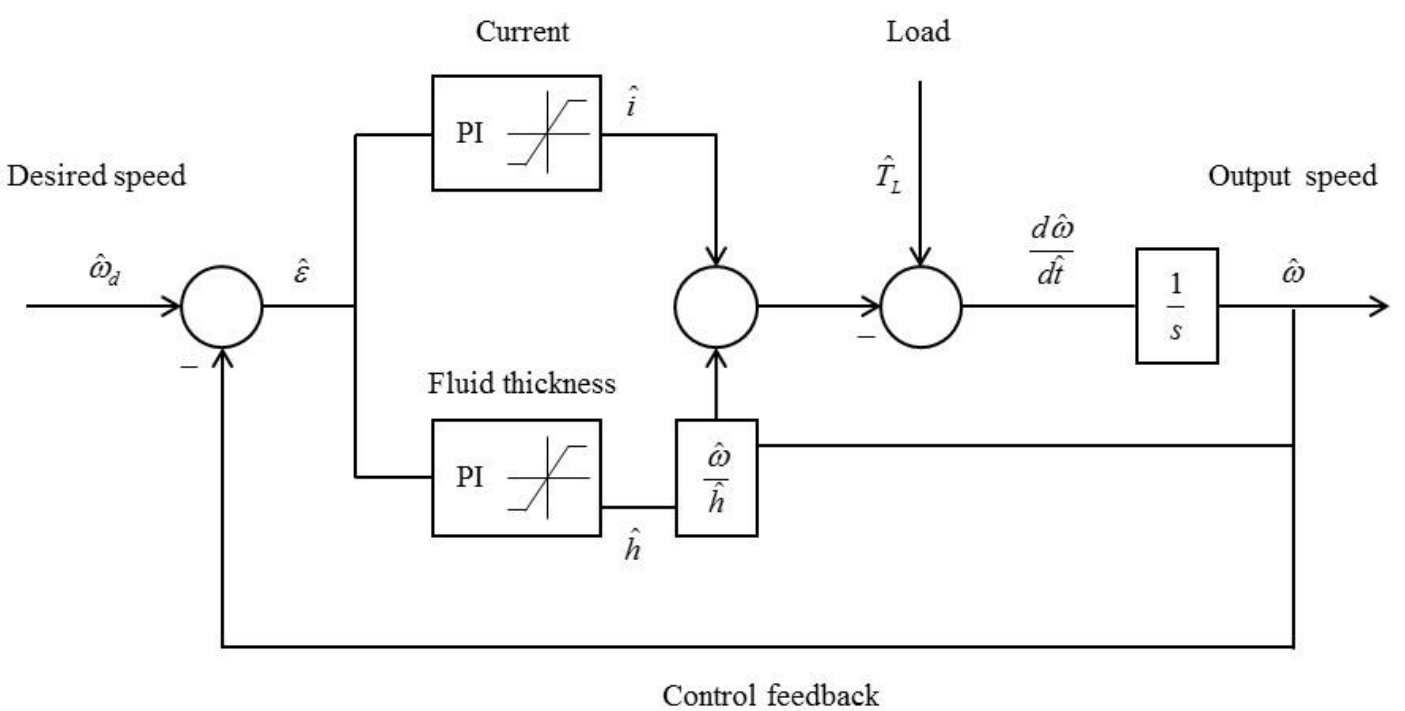

Figure 6-7. Block diagram for the MR fluid brake control showing two PI controllers with saturation and a nonlinear relationship for $\hat{h}$ 
The desired speed for the rotating system is shown in Fig. 6-7 by the symbol $\hat{\omega}_{d}$ and the system error is given by $\hat{\varepsilon}$. Again, the carets indicate that these are nondimensional quantities. The Laplace operator $1 / s$ is shown in Fig. 6-7 to denote integration.

Due to physical constraints, the fluid thickness $\hat{h}$ and the coil current $\hat{i}$ are likely to saturate during extreme speed conditions. For instance, a linear actuator will be used to control the MR fluid thickness between a maximum and minimum value allowed by the brake. For very slow speeds it will be desirable for $\hat{h}$ to be as small as possible in order to maximize the non-energized(off-field) brake torque; however, the fluid thickness cannot be allowed to approach zero. A minimum non-zero value for $\hat{h}$ is required to keep the rotating disk from making metal-to-metal contact with the stationary surface during operation. Similarly, a maximum value for the fluid thickness is needed during high speed operation, however, the maximum value for $\hat{h}$ is limited by the maximum stroke length for the linear actuator. For our study the nondimensional MR fluid thickness will be bounded between 0.1 and 3. As previously noted, we want the input current to remain within the linear region of the magnetic-flux density curve and should therefore not exceed the dimensional value of $i_{\max }$ where the nonlinearity in this curve begins [156]. Furthermore, we will limit the minimum allowable current to zero since there is no physical advantage of reversing the direction of current through the coil. In other words, the physical constraints for the fluid thickness and the electric current are given by

$$
0.1<\hat{h}<3 \text { and } 0<\hat{i}<1 \text {. }
$$

These saturation characteristics are shown graphically in Fig. 6-7 and will be used in the following paragraphs to describe the overall control design. 


\subsubsection{PI Controllers}

In order to simultaneously adjust the fluid thickness and the electric current for regulating system speed, a saturating PI control is chosen for both adjustments. The proportional control is the basic controller, while the integral control is added to ensure a zero steady-state error while regulating a constant speed.

Mathematically, the PI control law for adjusting the fluid thickness is given by

$$
\hat{h}=\hat{\mathrm{K}}_{p} \hat{\varepsilon}+\hat{\mathrm{K}}_{i} \int \hat{\varepsilon} d \hat{t}
$$

where $\hat{\mathrm{K}}_{p}$ and $\hat{\mathrm{K}}_{i}$ are the nondimensional proportional gain and integral gain respectively, and the nondimensional error is given by

$$
\hat{\varepsilon}=\hat{\omega}_{d}-\hat{\omega}
$$

As previously noted, Eq. (6.21) is used to calculate the controlled fluid-thickness within the bounds noted in Eq. (6.20). If Eq. (6.21) computes a fluid thickness that exceed 3 then the fluid thickness is simply set to 3. On the other hand, if Eq. (6.21) computes a fluid thickness that is less than 0.1 then the fluid thickness is set to 0.1 . In order to prevent integrator windup within the PI control during saturation, the integral gain is turned on and off as follows:

$$
\hat{\mathrm{K}}_{i}=\left\{\begin{array}{cc}
0 & \text { Eq. }(6.21)<0.1 \\
\hat{\mathrm{K}}_{i} & 0.1<\text { Eq. }(6.21)<3 \\
0 & 3<\text { Eq. }(6.21)
\end{array} .\right.
$$

As previously noted, the overall output of the fluid-thickness control is limited by the bounds shown in Eq. (6.20).

Similarly, the PI control law for adjusting the electric current is given by

$$
\hat{i}=-\hat{\Phi}_{p} \hat{\varepsilon}-\hat{\Phi}_{i} \int \hat{\varepsilon} d \hat{t}
$$

where $\hat{\Phi}_{p}$ and $\hat{\Phi}_{i}$ are the nondimensional proportional and integral gain, respectively 
and the nondimensional error is given in Eq. (6.22). Again, Eq. (6.24) is used to calculate the controlled current within the bounds noted in Eq. (6.20). If Eq. (6.24) computes a current that exceeds unity, then the current is simply set to 1 . On the other hand, if Eq. (6.24) computes a current that is less than zero, then the current is set to 0 . Once again, in order to prevent integrator windup within the PI control during saturation, the integral gain is turned on and off as follows:

$$
\hat{\Phi}_{i}=\left\{\begin{array}{cc}
0 & \text { Eq. }(6.24)<0 \\
\hat{\Phi}_{i} & 0<\text { Eq. }(6.24)<1 \\
0 & 1<\text { Eq. }(6.24)
\end{array} .\right.
$$

And again, the overall output of the current control is limited by the bounds in Eq (6.20).

\subsubsection{Non-Unique Solutions}

By setting the left-hand-side of Eq. (6.17) to zero, the steady-state solution for the speed of the system may be determined as a function of the MR fluid thickness and the current that is applied to the electric coil. This solution is given by

$$
\hat{\omega}=\left(\hat{T}_{L}-\hat{i}\right) \hat{h}
$$

The purpose for presenting the steady-state result is to illustrate that there is no unique combination of MR fluid thickness and electric current that establishes the steadystate condition for the brake. Rather, an infinite number of combinations exist within the bounds of operation that are stipulated in Eq. (6.20). Subsequent discussions relative to some results show that our controller always finds a solution with zero steady-state error; however, the exact combination of $\hat{h}$ and $\hat{i}$ depends upon the initial conditions from which the controller begins seeking this solution, and whether or not any saturation points are encountered along the way. 


\subsubsection{Linearization and Control-Gain Selection}

The controlled system that has been described in this dissertation describes a nonlinear system that is partly discontinuous. In order to check for some level of stability and to provide guidance for selecting control gains, it is helpful to linearize the system and to examine cases when no saturation limits are reached. For linearized conditions where $\hat{\omega} \approx 1$ and $\hat{h} \approx 1$, and where the two PI controls in Eq.s (6.21) and (6.24) are used, it may be shown that the dynamic response for the regulated system is governed by the following second-order differential equation:

$$
\frac{d^{2} \hat{\omega}}{d \hat{t}^{2}}+\left(1+\hat{\mathrm{K}}_{p}+\hat{\Phi}_{p}\right) \frac{d \hat{\omega}}{d \hat{t}}+\left(\hat{\mathrm{K}}_{i}+\hat{\Phi}_{i}\right) \hat{\omega}=\left(\hat{\mathrm{K}}_{i}+\hat{\Phi}_{i}\right) \hat{\omega}_{d}
$$

Equation (6.27) shows that the integral controllers successfully eliminate the steadystate error. This is shown by setting the time derivatives in Eq. (6.27) to zero and noting that $\hat{\omega}=\hat{\omega}_{d}$. Although not proven here, this property holds true even for the nonlinear system as demonstrated later in the simulation studies.

Another thing to observe from Eq. (6.27) is that all of the coefficients on the left-hand-side are positive, which means that the linearized and continuous system is stable. Although we present no proof of stability for the nonlinear system, the exercised nonlinear model appears to be robust and has demonstrated no instabilities in our study thus far. Again, this will be demonstrated in the simulation studies.

Finally, by inspecting Eq. (6.27) it may be shown that the nondimensional undamped natural-frequency and the damping ratio for the linearized system are given by

$$
\hat{\omega}_{n}=\sqrt{\hat{\mathrm{K}}_{i}+\hat{\Phi}_{i}} \quad \text { and } \quad \zeta=\frac{1+\hat{\mathrm{K}}_{p}+\hat{\Phi}_{p}}{2 \sqrt{\hat{\mathrm{K}}_{i}+\hat{\Phi}_{i}}}
$$

In order to provide an adequate rise time for the transient response, the 
nondimensional undamped natural-frequency will be designed so that $\hat{\omega}_{n}=4$.

Similarly, we will design the damping ratio such that $\zeta=\sqrt{2} / 2$. The following gain selections satisfy these conditions and will be used in the simulations which follow: $\hat{\mathrm{K}}_{p}=2.33, \hat{\mathrm{K}}_{i}=4, \hat{\Phi}_{p}=2.33$, and $\hat{\Phi}_{i}=12$.

\subsection{Summary}

In this chapter, a general introduction about the conventional frictional brake and MR fluid brake is presented. The principle of work of suggested CVFTs MR fluid brakes and how they are connected to the actuator is showed in detail. The mathematical model includes both mechanical and electrical parts are presented as well. MR fluid brake control includes. Description to the proposed MR fluid brake, dynamic analysis of the flywheel needed to be controlled is illustrated. Next, nondimensional analysis is carried out to generalize the model and make it more efficient. Finally, linearization are control gains selections are clearly illustrated. Important results and their discussions will be presented in the next chapter. 


\section{CHAPTER 7. REULTS AND DISCUSSION}

\subsection{General}

In the previous chapters, the governing equations of the conventional drum and magnetorheological (MR) fluid brakes are presented in detail, maximum pressure, and torque due drum friction, fluid viscosity and magnetic field. In this chapter, results of the sensitivity analyses for both conventional and magnetorheological fluid brake and control are explained and discussed in detail. This chapter is divided into three parts. The first part involves sensitivity results of drum brake, the second part involves the sensitivity results of the MR fluid brake, and finally control results. In the second part, sensitivity results are presented for mechanical and electrical design points of view. Depending on these results, one can indicate and specify the most important parameters that have a critical impact on the design and can be easily altered to get reasonable design (it is hard to change some design parameters throughout the design and operational conditions). Significant and insignificant design parameters can be determined from the results as well. The sensitivity results can be used as an important step for optimization studies after getting the most effective design parameters and work on it to enhance the overall performance of the brake design. The third part includes controlling of the magnetorheological fluid brake for high, medium, and low speed.

\subsection{Sensitivity Analysis of Conventional Drum Brake}

Figures 7-1 through 7-3 show plots of the sensitivity results in Eq. (3.15) as they vary with changes in $\hat{b}, \hat{c}$, and $\gamma$. These plots have been made for nominal values 


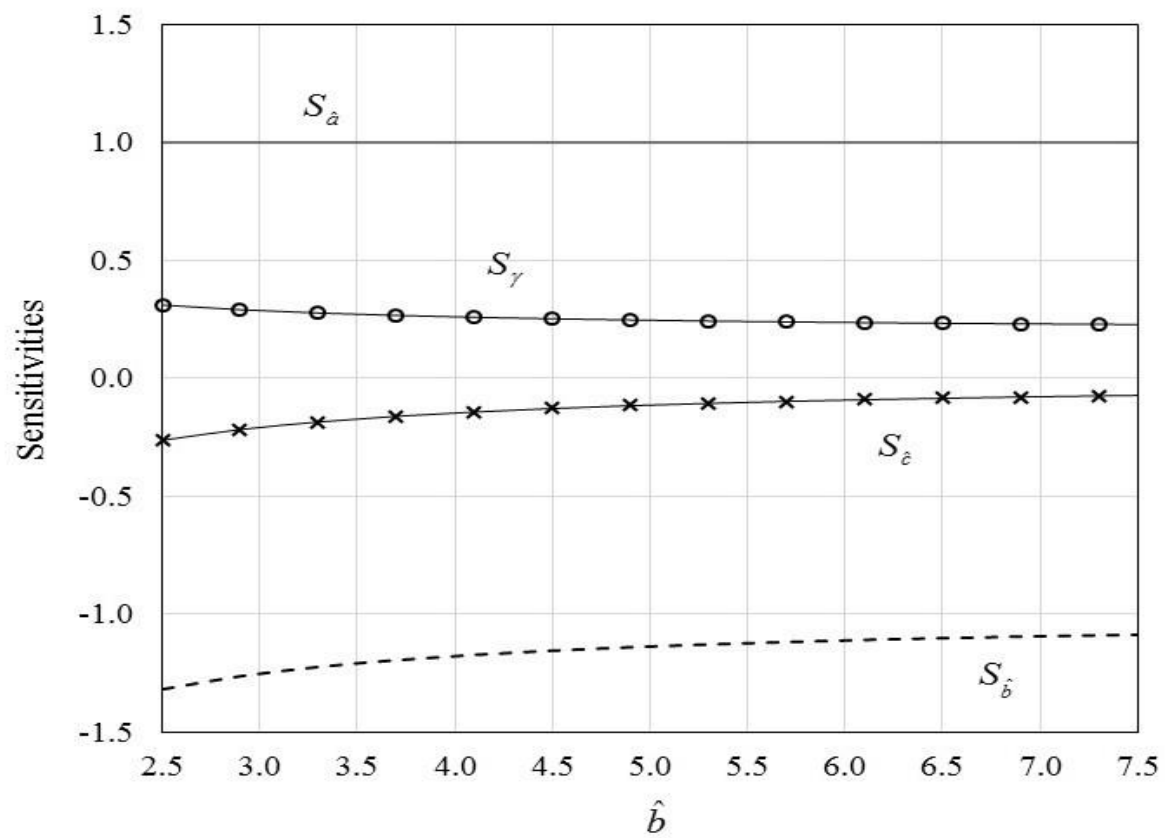

Figure 7-1. Variation in sensitivity coefficients for changes in $\hat{b}$. These results have been generated for nominal values of $\hat{c}=0.5$ and $\gamma=\pi / 2$

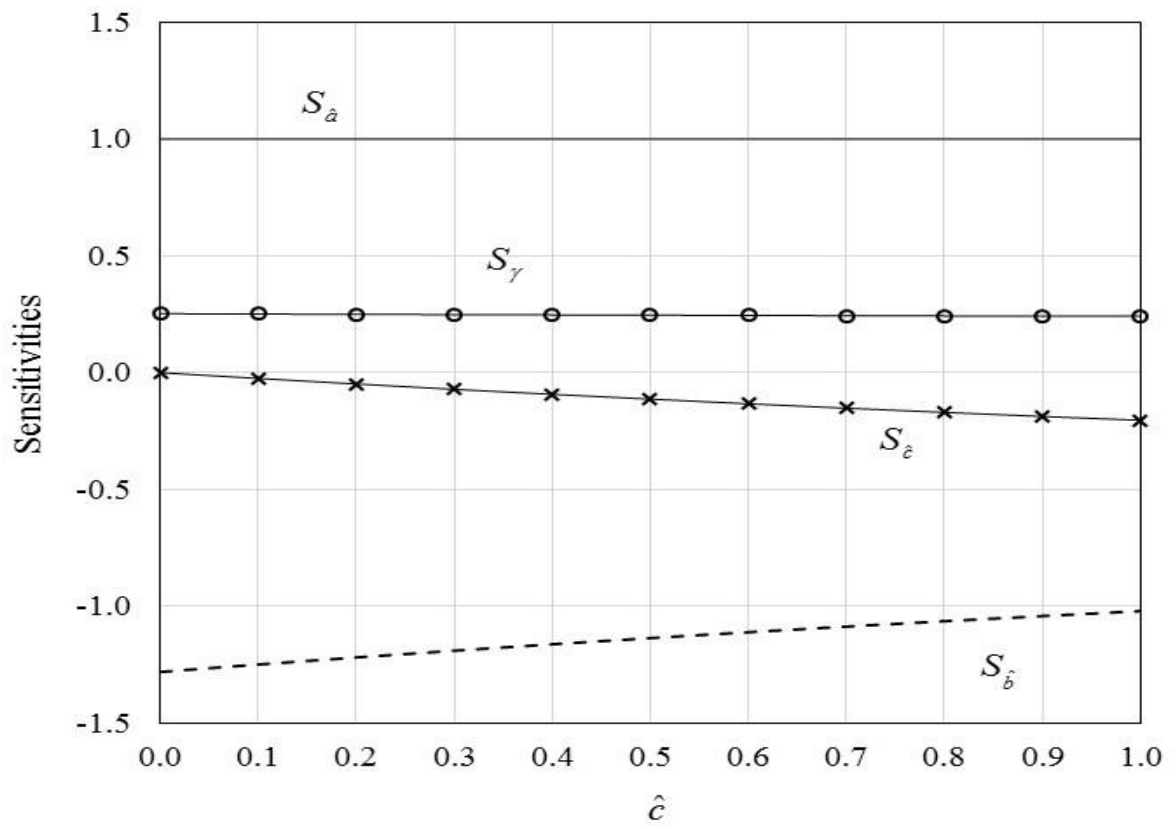

Figure 7-2. Variation in sensitivity coefficients for changes in $\hat{c}$. These results have been generated for nominal values of $\hat{b}=5$ and $\gamma=\pi / 2$ 


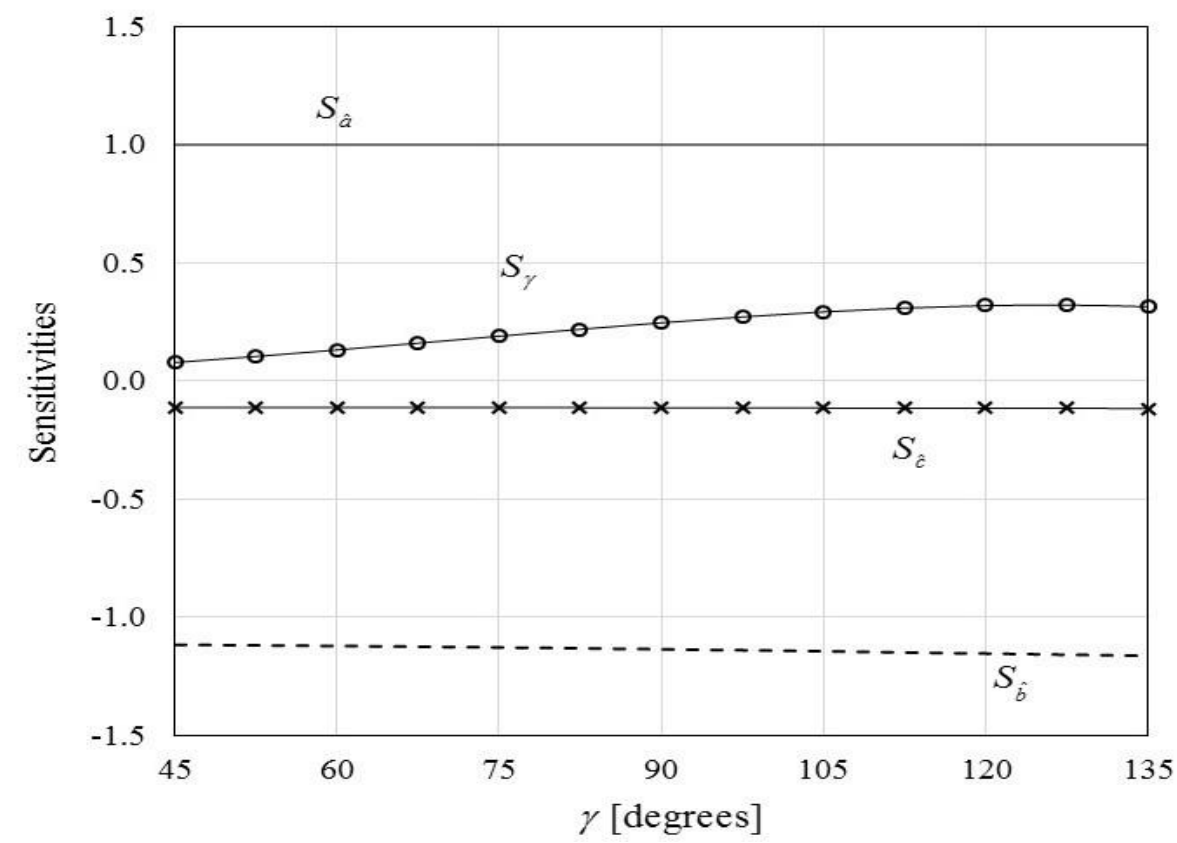

Figure 7-3. Variation in sensitivity coefficients for changes in $\gamma$ (reported in degrees). These results have been generated for nominal values of $\hat{b}=5$ and $\hat{c}=0.5$.

of $\hat{b}=5, \hat{c}=0.5$, and $\gamma=\pi / 2$. Note: None of these results vary with $\hat{a}$ but a scaled view of Fig. 3-1 shows that a nominal value for this parameter would be 2.5. As shown in Fig.s 7-1 through 7-3, the two parameters that demonstrate the greatest sensitivities are $\hat{a}$ and $\hat{b}$ as both sensitivity coefficients have magnitudes near unity. By increase the parameter $\hat{a}$, the braking torque increases proportionally. What is not so obvious is that by increasing $\hat{b}$, the braking torque decreases in almost a proportional manner as well. The decreasing torque results from the negative sign on the sensitivity coefficient and may also be seen from the fact that $\hat{b}$ appears in the denominator of Eq. (3.12) whereas $\hat{a}$ appears in the numerator.

Physically speaking, increasing $\hat{a}$ or decreasing $\hat{b}$ has the effect of increasing the contact pressure between the brake pad and the brake drum, thereby increasing the 
braking torque. In making these adjustments, the design engineer must keep in mind that this contact pressure cannot exceed the yield strength of the brake pad; otherwise the brake pad will undergo a mechanical failure. Another important conclusion to be drawn from Fig.s 7-1 through 7-3 is that adjustments in $\hat{c}$ and $\gamma$ produce a negligible impact on the braking torque of the drum brake compared to adjustments in $\hat{a}$ and $\hat{b}$. Therefore, when seeking to increase the braking torque, the design engineer should emphasis design changes for $\hat{a}$ and $\hat{b}$ over design changes in $\hat{c}$ and $\gamma$. Finally, it is worth noting that the sensitivity coefficients that are plotted Fig.s 7-1 through 7-3 remain fairly flat across the variation in a given parameter. In other words, there is not a tremendous change in the previous conclusions across a broad range of reasonable adjustments in a given drumbrake parameter. This fact may be used to generalize our conclusions for drum brakes with any facsimile to the design shown in Fig. 3-1.

\subsection{Sensitivity Analysis of MRF Brake}

\subsubsection{Viscous and Magnetic Field Braking Torque}

Figures 7-4 through 7-9 show plots of the sensitivity results in Eq.s (3.65) through (3.68) as they vary with changes in $\hat{r}_{i o}, \hat{\beta}_{o}, \hat{k}_{o}$, and $\hat{H}_{o}$ for both $\hat{T}_{p}$ and $\hat{T}_{H}$, respectively. Fig. 7-4 shows sensitivity variations with $\hat{r}_{i o}$ for a positive range of the nondimensional radius. One vertical asymptote is noticed at $\hat{r}_{i o}=1$ making $S_{\hat{r}_{i}}$ value undefined. However, it makes no physical meaning when $\hat{r}_{i o}$ negative, equal or greater than unity. Physically, $\hat{r}_{i o}$ is the ratio between brake radii $\left(\hat{r}_{i}=r_{i} / r_{o}\right)$ and none of them can be negative (cannot be considered as a reasonable physical assumption for 
MRF brake).

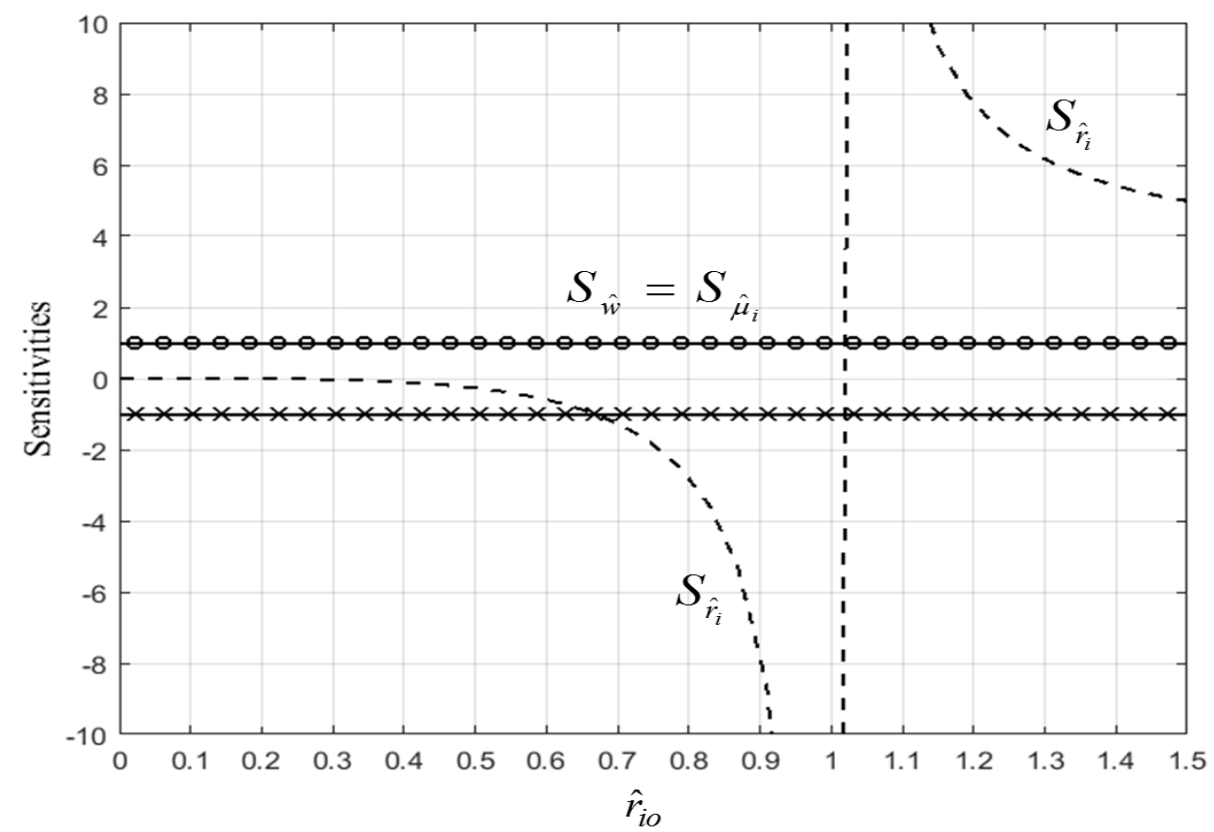

Figure 7-4. Variation in sensitivity coefficients for changes in nondimensional radius $\hat{r}_{i}$. For nondimensional $\left(\hat{T}_{P}\right)$. These results have been generated for positive nominal values only.

As shown in Fig 7-4, the three parameters that demonstrate the greatest sensitivities for a particular change of $\hat{r}_{i o}$ (up to 0.7 ) are $\hat{w}, \hat{\mu}$, and $\hat{h}$ as their sensitivity coefficients have unity magnitudes. That means by increase the parameters $\hat{w}$ and $\hat{\mu}$, the braking torque increases proportionally, while increasing $\hat{h}$ decreases braking torque proportionally.

Physically speaking, increasing $\hat{h}$ has the effect of decreasing fluid shear strain and then decreasing plastic fluid shear stress. The decreasing torque is resulted from the negative sign on the sensitivity coefficient and may also be seen from the fact that $\hat{h}$ appears in the denominator of Eq. (3.31) whereas $\hat{\mu}$ and $\hat{\omega}$ appears in the numerator. By increasing the 
parameter $\hat{r}_{i o}$ sensitivity $S_{\hat{r}_{i}}$ and the braking torque decreases nonlinearly. Torque decreases in different trend so that the change in torque reduction become noticeable after $\hat{r}_{i o}=0.6$ and greater. Physically speaking, increasing $\hat{r}_{i o}$ has the effect of increasing the inner disc radius which leads to reduce the frictional surface area and then reducing the braking torque.

Another important conclusion to be drawn from Fig. 7-4 is that adjustments in $\hat{w}, \hat{\mu}$, and $\hat{h}$ has a remarkable impact on the viscous braking torque of MRF brake compared to adjustments in $\hat{r}_{i o}$ within $0-0.6$ change in the previous conclusions across a broad range of reasonable adjustments.

As shown in Fig. 7-5, the parameter that demonstrates greatest sensitivities for a particular change in $\hat{r}_{i o}$ is $\hat{k}$ as its sensitivity coefficient has unity magnitude.

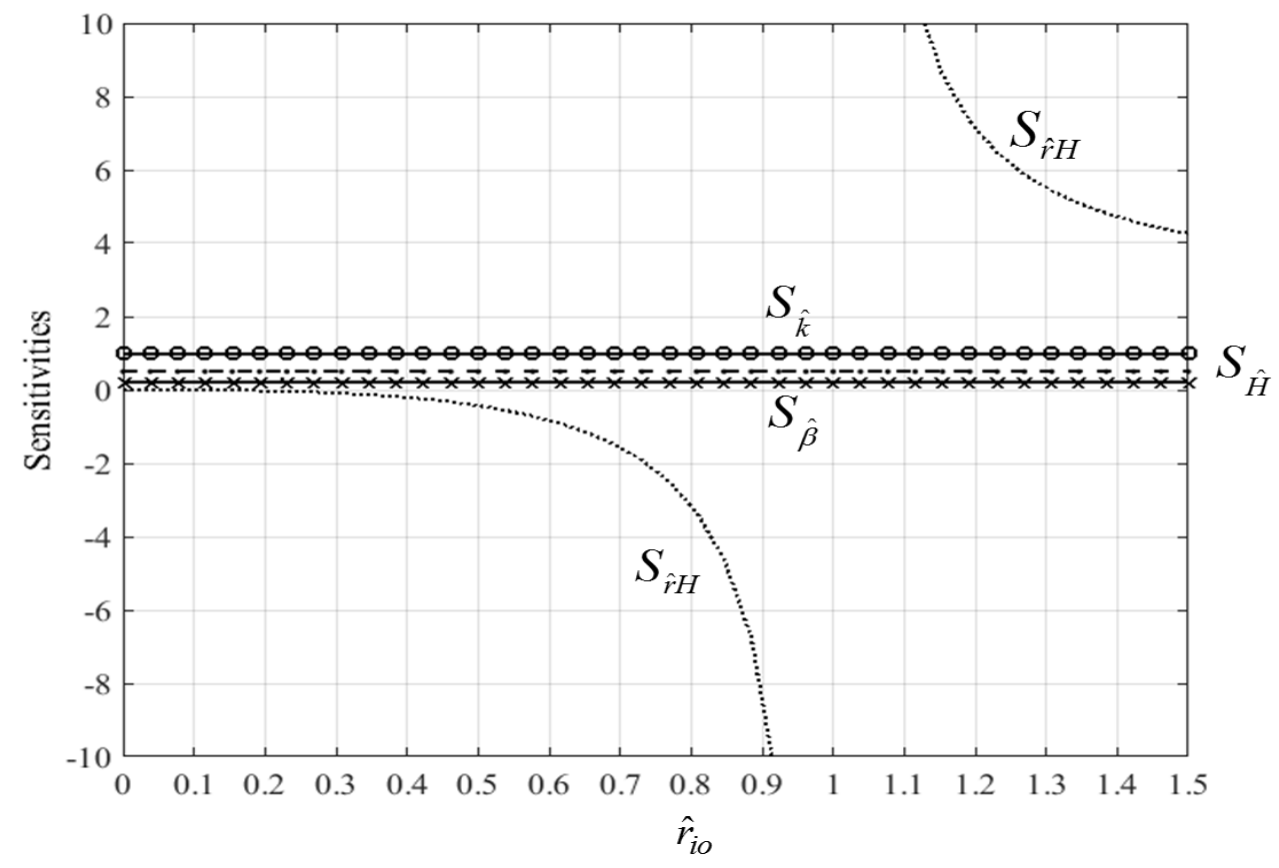

Figure 7-5. Variation in sensitivity coefficients for changes in nondimensional radius $\hat{r}_{i}$ for the nondimensional torque $\left(\hat{T}_{H}\right)$. These results have been generated for nominal values of $\hat{k}=0.5, \hat{\beta}=0.5$, and $\hat{H}=1.5$ 
By increase $\hat{k}$ parameter the braking torque increases proportionally, while increasing $\hat{r}_{i}$ the braking torque decreases nonlinearly.

As shown in Fig. 7-6, the parameter that demonstrates greatest sensitivities for a

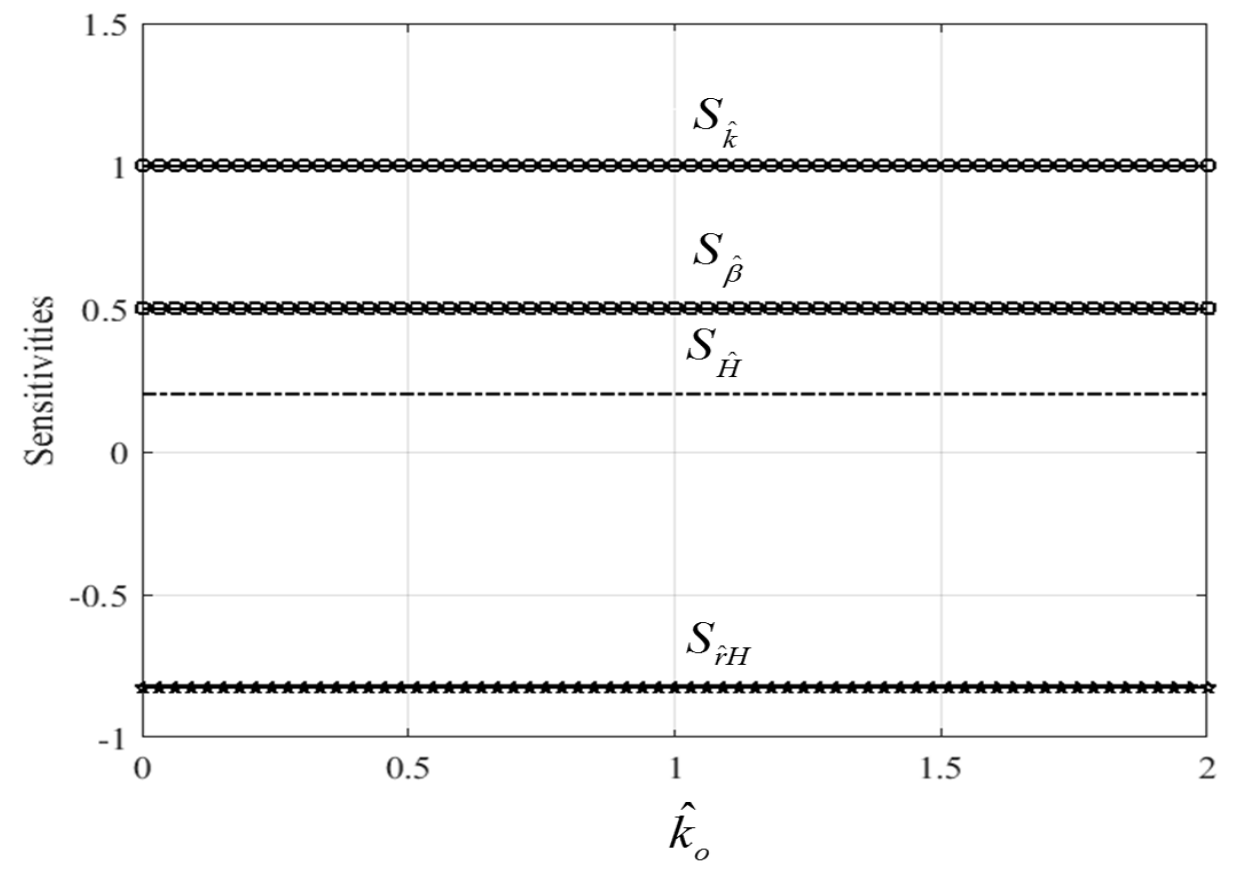

Figure 7-6. Variation in sensitivity coefficients for changes in nondimensional $\hat{k}_{o}$ for the nondimensional torque $\left(\hat{T}_{H}\right)$. These results have been generated for positive nominal values $\hat{r}_{i}=0.6, \hat{\beta}=0.5$, and $\hat{H}=1.5$

particular change in $\hat{k}_{o}$ is $\hat{k}$ as its sensitivity coefficient has unity magnitude. All sensitivities remain flat with increasing $\hat{k}_{o}$ and the braking torque increases proportionally with $\hat{\beta}, \hat{k}$, and $\hat{H}$, and decreases with $\hat{r}_{i}$.

Figure 7-7 shows the sensitivity coefficient variations for changes in nondimensional $\hat{\beta}_{o}$ for the nondimensional torque $\left(\hat{T}_{H}\right)$. Sensitivity coefficients $S_{\hat{k}}$ and $S_{\hat{r} H}$ remain flat and independent on variation of $\hat{\beta}_{o}$ while $S_{\hat{\beta}}$ and $S_{\hat{H}}$ vary almost linearly 
with increases $\hat{\beta}_{o}$.

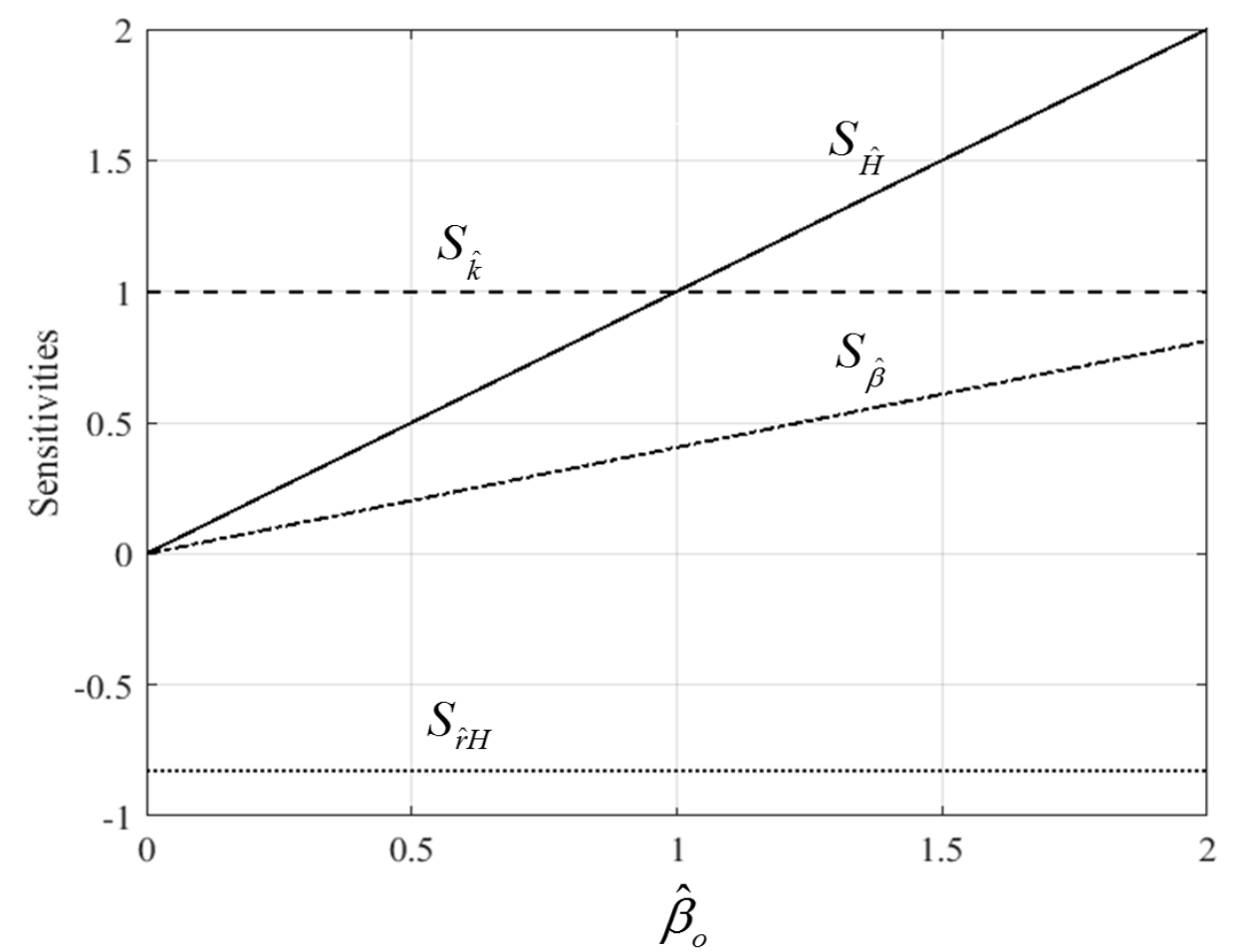

Figure 7-7. Variation in sensitivity coefficients for changes in nondimensional $\hat{\beta}_{o}$ for the nondimensional torque $\left(\hat{T}_{H}\right)$. These results have been generated for positive nominal values $\hat{r}_{i}=0.6, \hat{k}=0.5$, and $\hat{H}=1.5$

It can be seen that torque increasing due to sensitivity variation of $\hat{\beta}_{o}$ is greater than that corresponding of $\hat{H}$. That simply means effect of $\hat{\beta}$ is more dominant than $\hat{H}$.

As shown in Fig.s 7-8 through 7-9, the two parameters that demonstrate the greatest sensitivities are $\hat{\beta}$ and $\hat{k}$. The braking torque increases with increasing magnetic field and the exponent. However, when $\hat{H}$ values smaller than 1 Fig. 7-9, $S_{\hat{\beta}}$ decreases with increasing nondimensional parameter $\hat{\beta}_{o}$ and increases with increasing $\hat{\beta}_{o}$ when $\hat{H}$ 
value greater than unity.

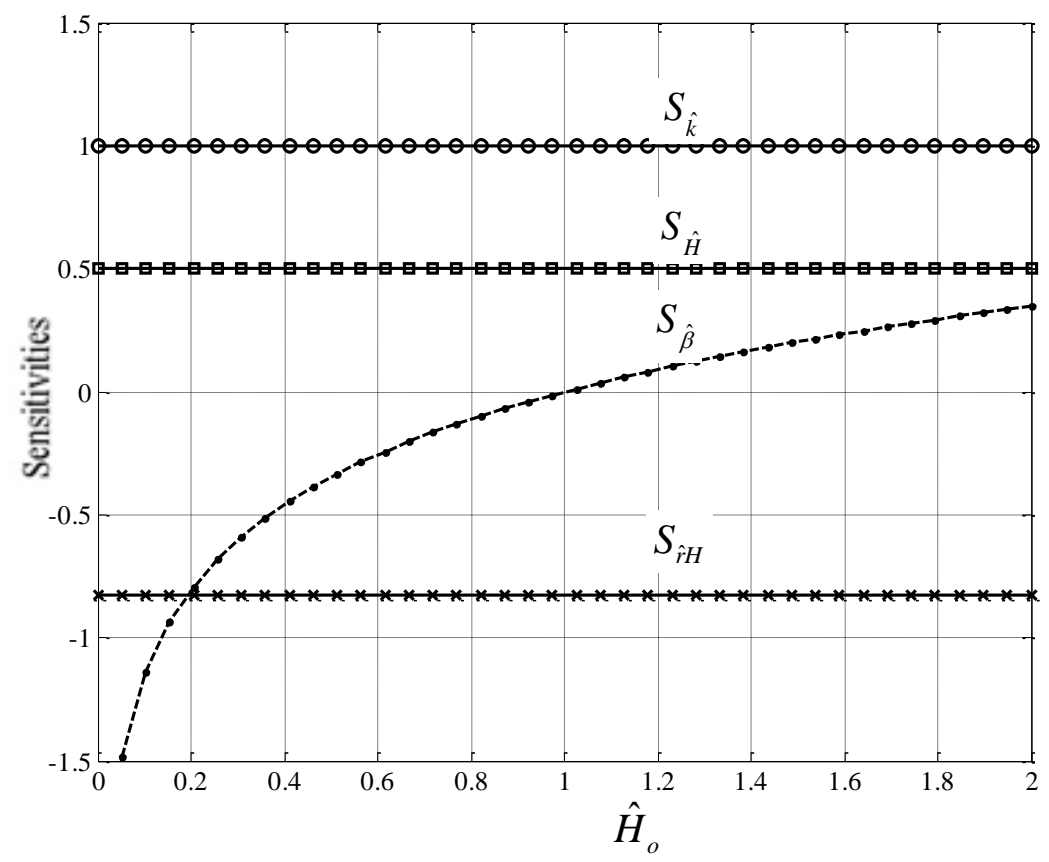

Figure 7-8. Variation in sensitivity coefficients for changes in nondimensional $\hat{H}_{0}$ for the nondimensional torque $\left(\hat{T}_{H}\right)$. These results have been generated for positive nominal values $\hat{r}_{i}=0.6, \hat{k}=0.5$, and $\hat{\beta}=0.5$.

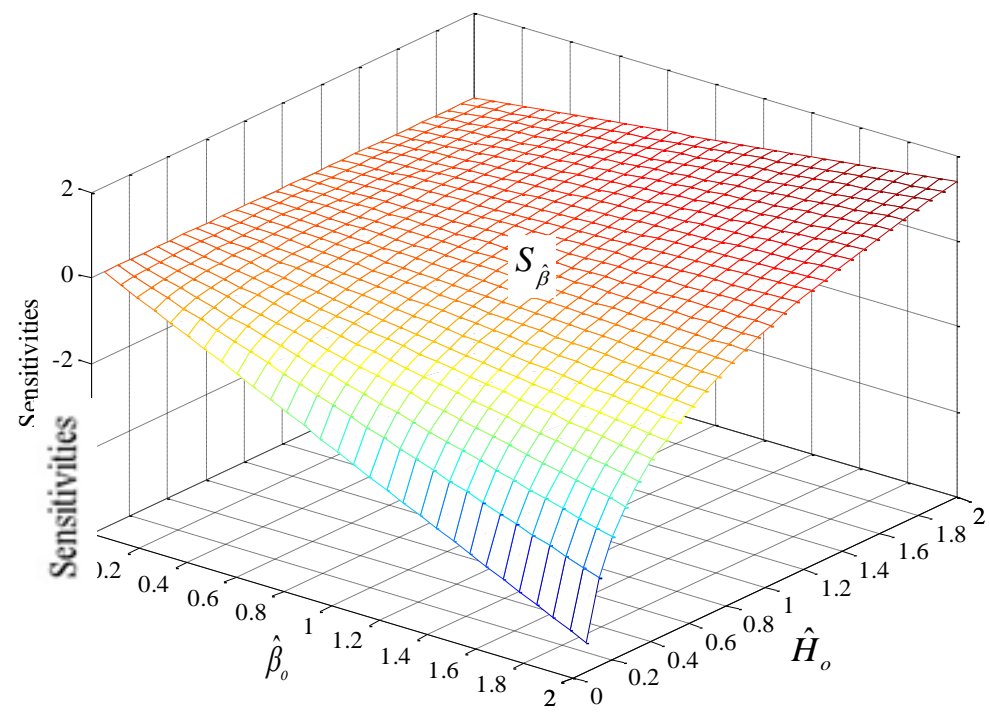

Figure 7-9. Variation in sensitivity coefficients for changes in nondimensional $\hat{H}_{0}$ for the nondimensional torque $\left(\hat{T}_{H}\right)$. These results have been generated for positive nominal values $\hat{r}_{i}=0.6$ and $\hat{k}=0.5$. 
In other words, at a critical value of the nondimensional $\hat{H}(\hat{H}=1)$, braking torque shows no sensitivity $\left(S_{\hat{\beta}}=0\right)$ regarding variations of the nondimensional parameter $\hat{\beta}_{o}$. This behavior can be noticed form Eq.s (3.62) and (3.68) which means whenever $\hat{H}=1$, $1^{\hat{\beta}_{o}}=1$ braking torque is no longer depends on changing of exponent $\hat{\beta}$.

Physically speaking, increasing $\hat{H}, \hat{\beta}$, and $\hat{k}$ or decreasing $\hat{r}_{i}$ has the effect of increasing the magnetic field properties and stronger chain-like structure through the magnetorheological fluid. Decreasing the inner radius or increasing the outer radius has the effect of increasing the frictional contact area between the rotating disc/discs and brake case, thereby increasing the braking torque. In making these adjustments, the design engineer must keep in mind that this increasing or decreasing these design parameters must be under control within specific limitation. For example, the designer cannot increase braking torque by increasing outer radius for the application of limited space. Another important conclusion to be drawn from figures above is that none of all design parameters for viscous and magnetic braking torque has a negligible effect. However, a designer must make a tradeoff between them to get maximum braking torque considering all constraints and physical limitations.

\subsubsection{Electromagnetic Circuit Characteristics of MRF brake}

Figures 7-10 through 7-15 show plots of the sensitivity results in Eq.s (3.76) through (3.85) as they vary with changes in $\hat{r}_{10}, \hat{r}_{20}, \hat{r}_{3 o}$, and $\hat{r}_{4 o}$ for $\hat{\mathfrak{R}}_{A}, \hat{\mathfrak{R}}_{B}, \hat{\mathfrak{R}}_{C}$, and $\hat{\mathfrak{R}}_{D}$ respectively. For the given values of the nondimensional parameter in Fig. 7-10, one vertical asymptote is noticed at $\hat{r}_{3 o}=1.7$ making $S_{A \hat{A}_{3}}$ and $S_{A \hat{r}_{4}}$ values not defined. 


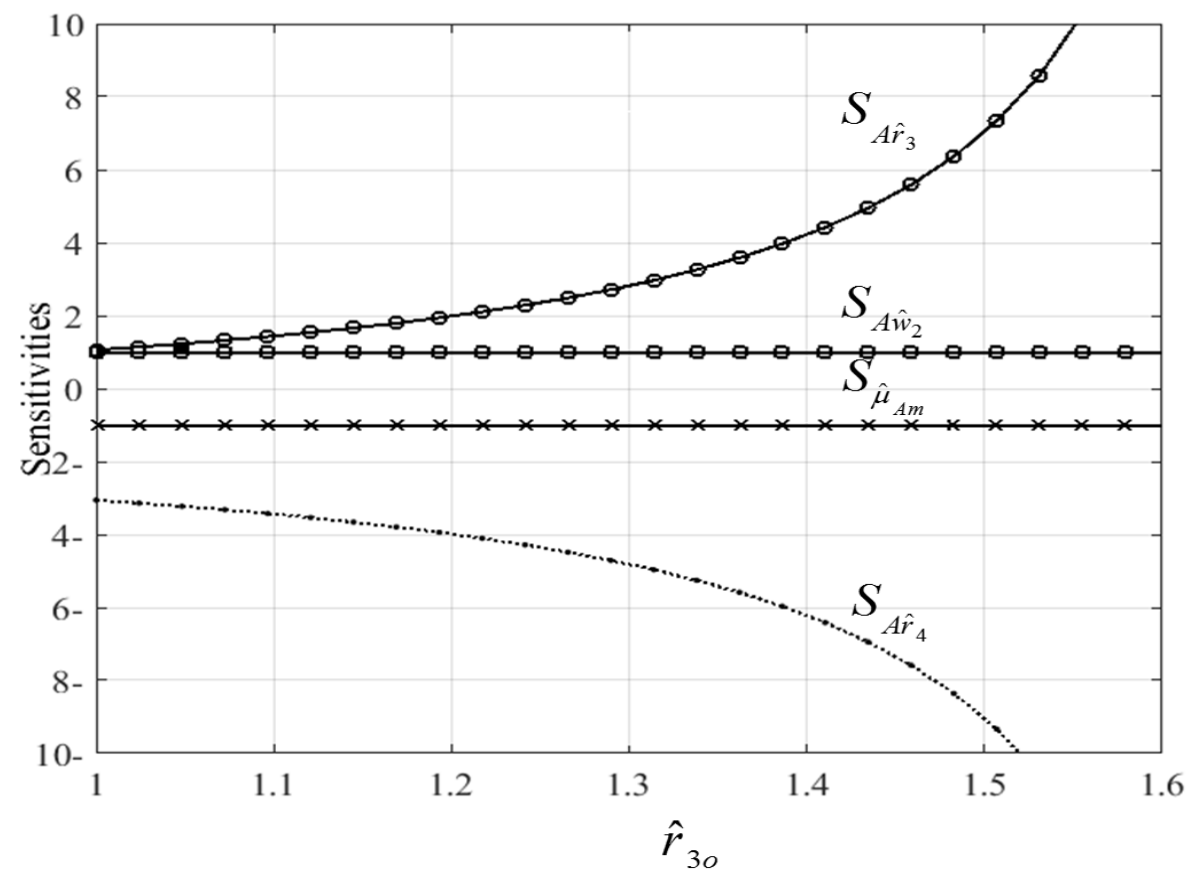

Figure 7-10. Variation in sensitivity coefficients for changes in nondimensional radius $\hat{r}_{3}$ for the nondimensional reluctance $\left(\hat{\mathfrak{R}}_{A}\right)$. These results have been generated for nominal values of $\hat{r}_{1}=0.6, \hat{r}_{2}=1$, and $\hat{r}_{4}=1.7$.

However, it makes no physical meaning when $\hat{r}_{30}$ is a negative value or unity. Physically speaking, $\hat{r}_{3 o}$ is the ratio between brake radii $\left(\hat{r}_{3 o}=r_{3 o} / r_{2 o}\right)$ as shown in Fig. 3-15 and none of them of can be negative. In addition, the nondimensional reluctance at section A, increases with $\hat{r}_{3 o}$ and $\hat{w}_{2}$ and decreases with $\hat{\mu}_{A m}$ and $\hat{r}_{4 o}$. The trend of increasing and decreasing of the reluctance with $\hat{w}_{2}$ and $\hat{\mu}_{A m}$ is linear as their sensitivities have a unity magnitude. That means by decreasing $\hat{w}$ and increasing $\hat{\mu}$, the magnetic reluctance decreases, and the corresponding braking torques due to the magnetic field increases proportionally. Increasing the reluctance $\hat{\mathfrak{R}}_{A}$ is resulted from the positive sign on the sensitivity coefficients and may also be seen from the fact that $\hat{r}_{30}$ appears in the 
denominator with the negative sign in Eq. (3.70). Physically speaking, increasing $\hat{r}_{3 o}$ has the effect of decreasing the cross-sectional area of the magnetic flux which lead to increase the magnetic reluctance through that section. Another important conclusion to be drawn from Fig. 7-10 is that all design parameters mentioned above have a remarkable impact on the magnetic reluctance and none of them can be neglected. Same conclusion can be noticed from Fig. 7-11 as the sensitivities vary with $\hat{r}_{40}$.

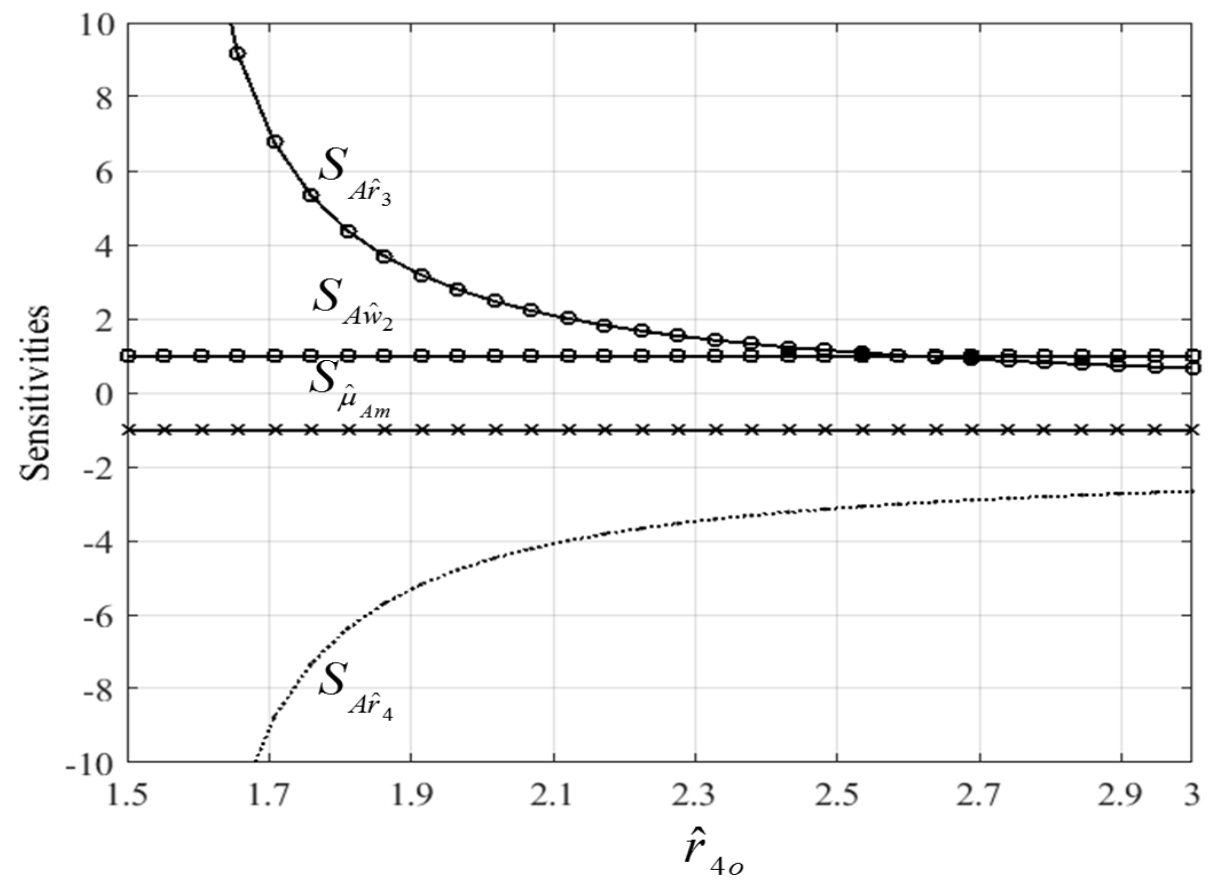

Figure 7-11. Variation in sensitivity coefficients for changes in nondimensional radius $\hat{r}_{4}$ for the nondimensional reluctance $\left(\hat{\mathfrak{R}}_{A}\right)$. These results have been generated for nominal values of $\hat{r}_{1}=0.6, \hat{r}_{2}=1$, and $\hat{r}_{3}=1.5$

Fig.s 7-12 and 7-13 show different response of the sensitivity values corresponding to the magnetic reluctance of the section B. As shown, $\hat{\mathfrak{R}}_{B}$ is linearly decrease as $\hat{\mu}_{B m}$ and $\hat{w}_{1}$ increase as they show a flat response with unity magnitude. 


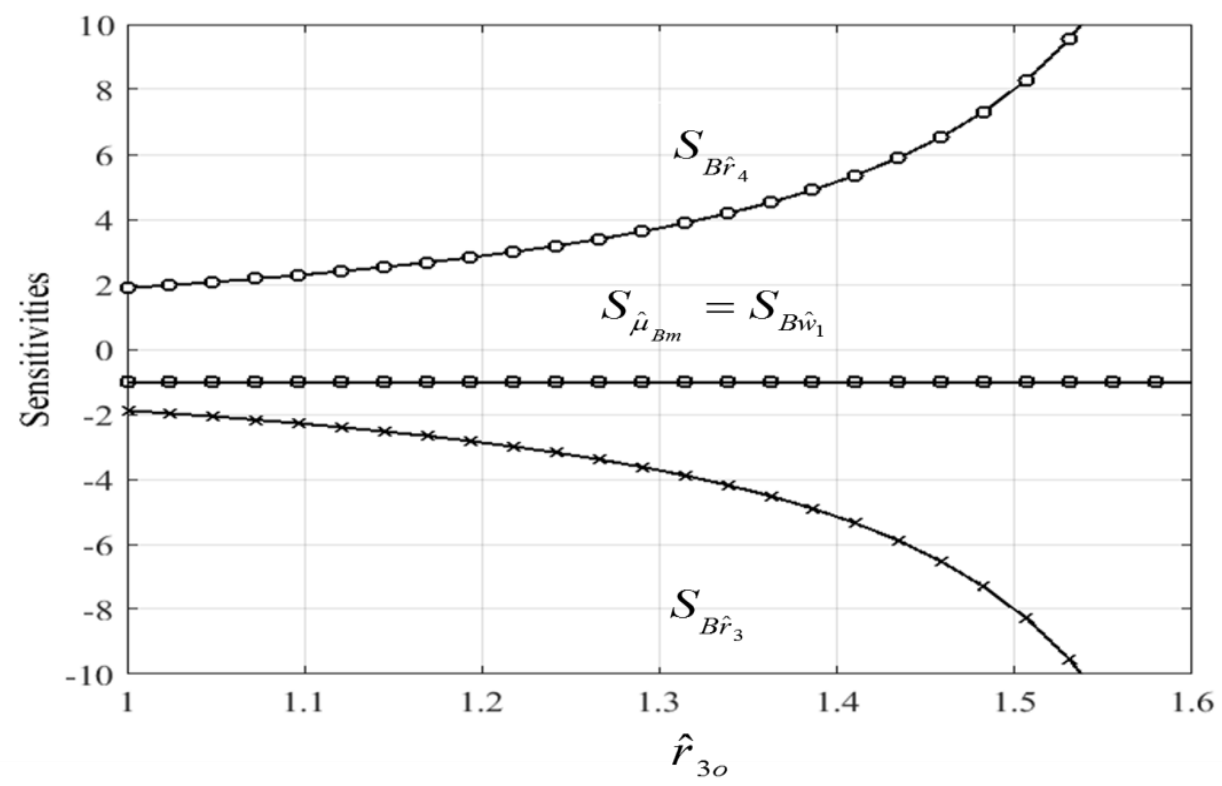

Figure 7-12. Variation in sensitivity coefficients for changes in nondimensional radius $\hat{r}_{3}$ for the nondimensional reluctance $\left(\hat{\mathfrak{R}}_{B}\right)$. These results have been generated for nominal values of $\hat{r}_{1}=0.6, \hat{r}_{2}=1$, and $\hat{r}_{4}=1.7$

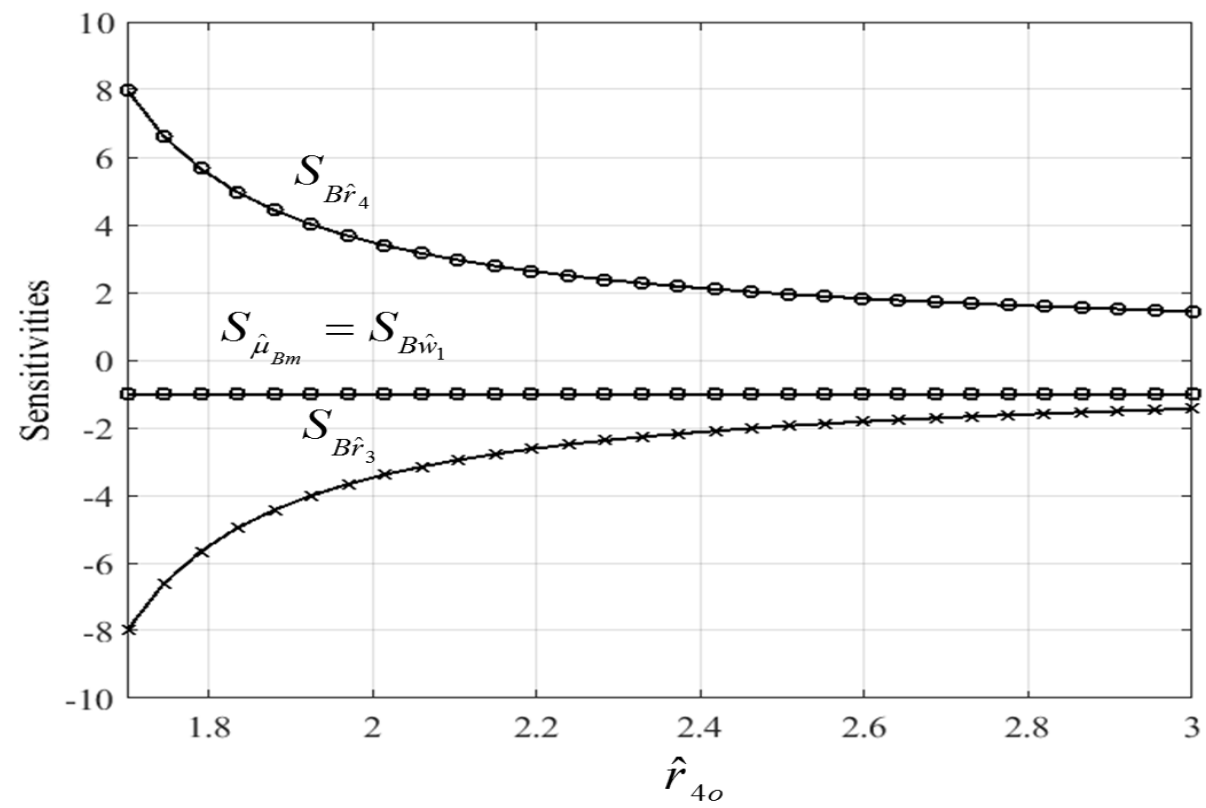

Figure 7-13. Variation in sensitivity coefficients for changes in nondimensional radius $\hat{r}_{3}$ for the nondimensional reluctance $\left(\hat{\mathfrak{R}}_{B}\right)$. These results have been generated for nominal values of $\hat{r}_{1}=0.6, \hat{r}_{2}=1$, and $\hat{r}_{3}=1.5$ 
However, $\hat{R}_{B}$ decreases with $\hat{r}_{3}$ and increases with $\hat{r}_{4}$. The increasing or decreasing rate of increment increases as $\hat{r}_{3}$ and $\hat{r}_{4}$ increase. This response can be seen from the fact that they appear in the numerator and denominator, respectively.

It is worth to do a simple comparison between Fig.s (7-10 and 7-11) and (7-12 and 7-13). This comparison reveals that nondimensional reluctance $\hat{\mathfrak{R}}_{A}$ decreases as $\hat{r}_{3}$ decrease while $\hat{\mathfrak{R}}_{B}$ decreases as $\hat{r}_{3}$ increase. That simply means $\hat{r}_{3}$ has a counteract effect as it increases one and decreases the other. However, to get better design of the electromagnetic circuit, a designer need to decrease the overall or equivalent magnetic reluctance as low as possible to increase the magnetic flux and reducing the dependency on the higher value of the input current to the electromagnetic coil to prevent the magnetic saturation problem. Therefore, when seeking to decrease the magnetic reluctances $\hat{\mathfrak{R}}_{A}$ and $\hat{\mathfrak{R}}_{B}$, a design engineer must make a tradeoff between them and then select the best values for $\hat{r}_{3}$ and $\hat{r}_{4}$ which make an equilibrium between the increasing in one of them and decreasing the other so that the net effect of them decreases the equivalent reluctance.

Fig.s 7-14 and 7-15 show the sensitivity coefficients variations for changes for the nondimensional reluctances $\hat{\mathfrak{R}}_{C}$ and $\hat{\mathfrak{R}}_{D}$, respectively. The figures show same behavior of the nondimensional reluctance $\hat{\mathfrak{R}}_{A}$ for the variations of $\hat{r}_{3}$ and $\hat{r}_{4}$ but the only difference is they vary with $\hat{r}_{1}$ and $\hat{r}_{2}$ instead of $\hat{r}_{3}$ and $\hat{r}_{4}$. As shown, the four parameters that demonstrate greatest sensitivities for a particular change of $\hat{r}_{1}$ (up to 0.6) are $\hat{h}, \hat{w}_{3}$, $\hat{\mu}_{C m}$, and $\hat{\mu}_{D m}$ as their sensitivity coefficients has unity magnitudes. 


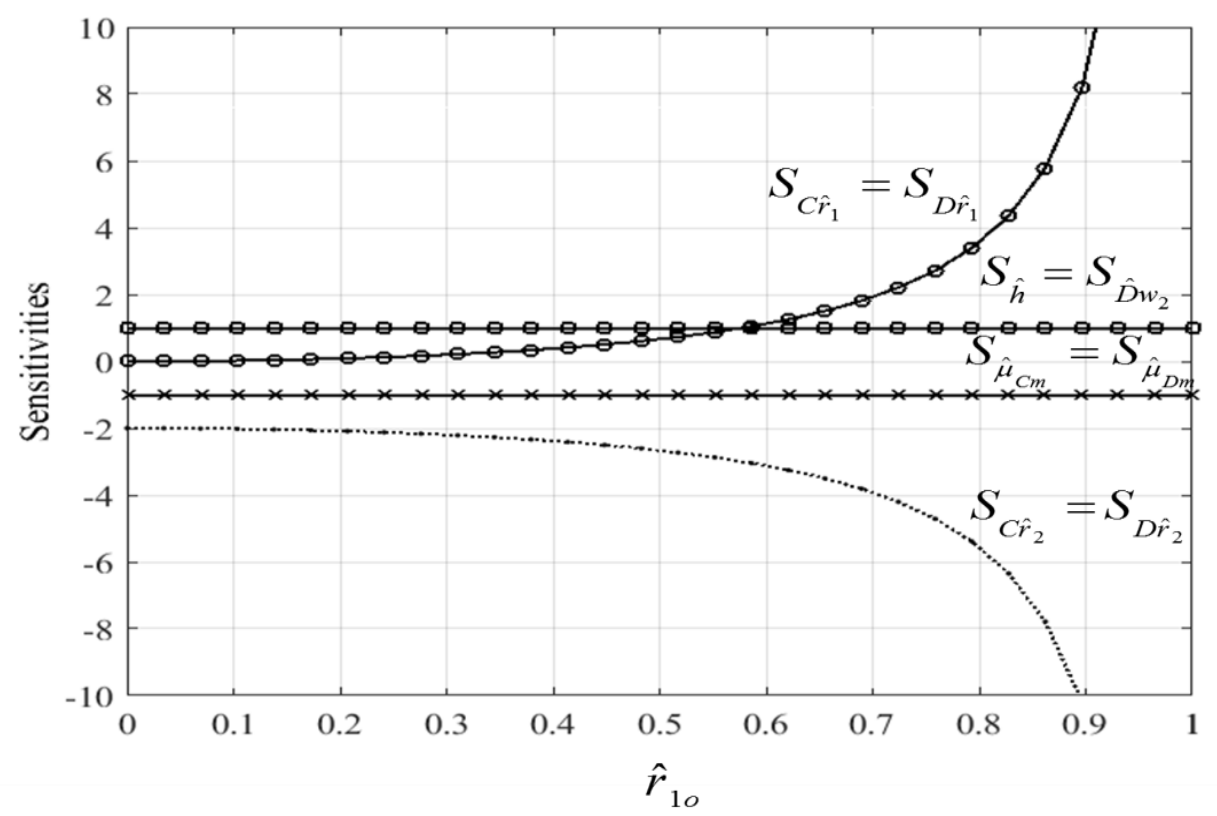

Figure 7-14. Variation in sensitivity coefficients for changes in nondimensional radius $\hat{r}_{1}$ for the nondimensional reluctance $\left(\hat{\mathfrak{R}}_{C}\right.$ and $\left.\hat{\mathfrak{R}}_{D}\right)$. These results have been generated for nominal values of $\hat{r}_{2}=1, \hat{r}_{3}=1.5$, and $\hat{r}_{4}=1.7$

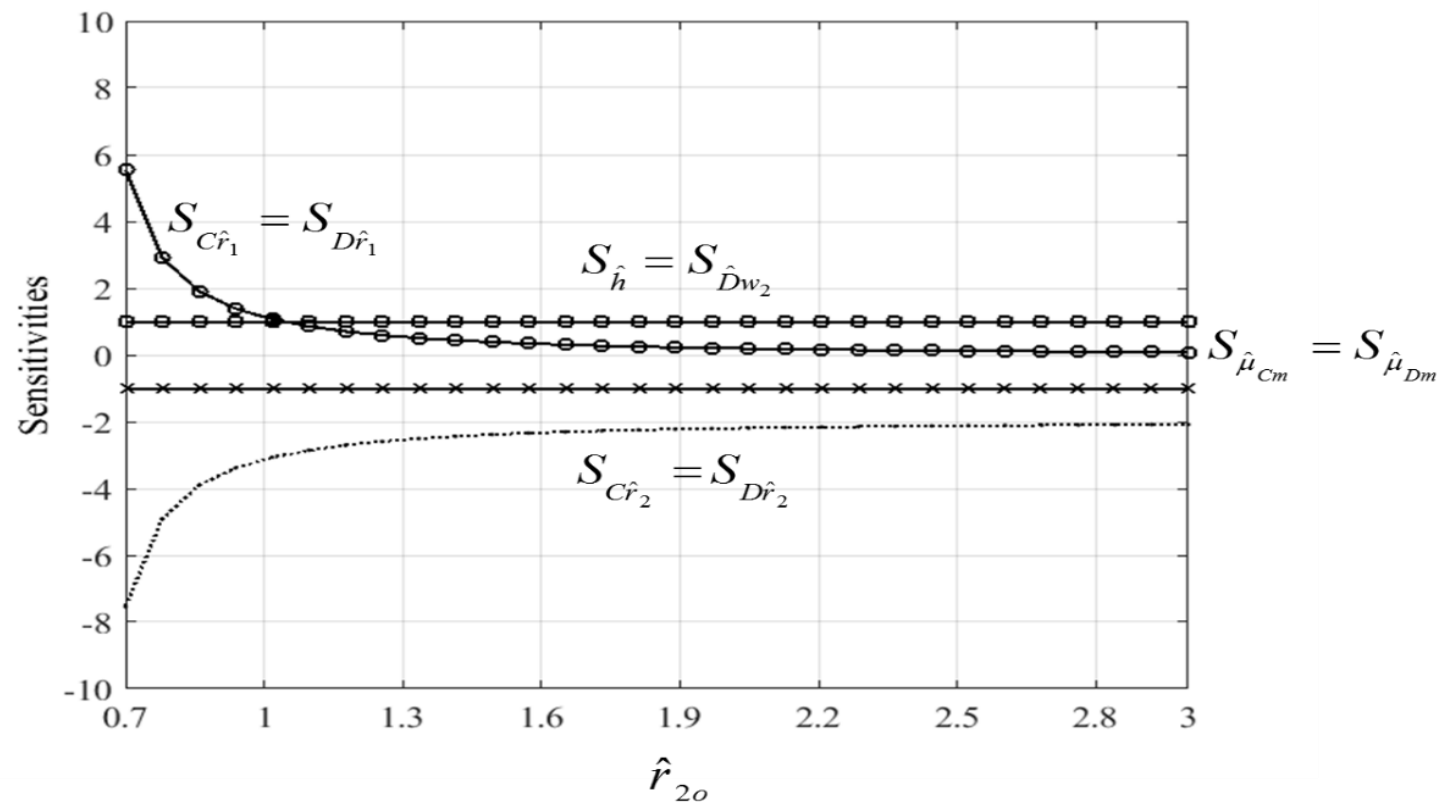

Figure 7-15. Variation in sensitivity coefficients for changes in nondimensional radius $\hat{r}_{2}$ for the nondimensional reluctance $\left(\hat{\mathfrak{R}}_{C}\right.$ and $\left.\hat{\mathfrak{R}}_{D}\right)$. These results have been generated for nominal values of $\hat{r}_{1}=0.6, \hat{r}_{3}=1.5$, and $\hat{r}_{4}=1.7$ 
By increasing the parameters $\hat{h}$ and $\hat{w}_{3}$, the magnetic reluctances at sections A and B increase and thereby the corresponding braking torque linearly decrease.

7.3.3 Combined Effect of Brake Radii $\hat{r}_{1}$ and $\hat{r}_{2}$ on Mechanical and Electromagnetic Circuit Designs of MRF Brakes

It is worth to notice that both $\hat{r}_{1}$ and $\hat{r}_{2}$ play a very important role in the equivalent reluctances values as well as the braking torque due to viscosity and magnetic field effects, $\hat{T}_{P}$ and $\hat{T}_{H}$, respectively. In other words, the equivalent magnetic reluctance decreases, and the braking torque increases as the inner radius $\hat{r}_{1}$ decrease or $\hat{r}_{2}$ increase. That means the variations of these two design parameters have same effect of both mechanical and electromagnetic characteristics of the MRF brake. These effects can be seen form Fig.s 7-4 and 7-5 for braking torque and Fig.s 7-14 and 7-15 for magnetic reluctance. Similar behavior can be seen for the design parameter $\hat{h}$ which appears in both mechanical and electromagnetic circuit design as well. As the fluid thickness decreases, the magnetic reluctance of section $\mathrm{C}$ decrease (which decreases the equivalent magnetic reluctance, increasing the magnetic flux, and thereby increasing the braking torque due to magnetic field, $\hat{T}_{H}$ ) and increase the braking torque due to fluid viscosity, $\hat{T}_{P}$. This is another benefit of the new design of the magnetorheological fluid brake for one and two sides of fluid variation presented in Ch.5.

7.4 Controlling a Magnetorheological (MR) fluid brake.

\subsubsection{High Speed Control.}

Figure 7-16 represents a transient response for the controlled brake system where 
the applied load torque is given by $\hat{T}_{L}=1$ and the desired shaft speed is given by $\hat{\omega}_{d}=2$.

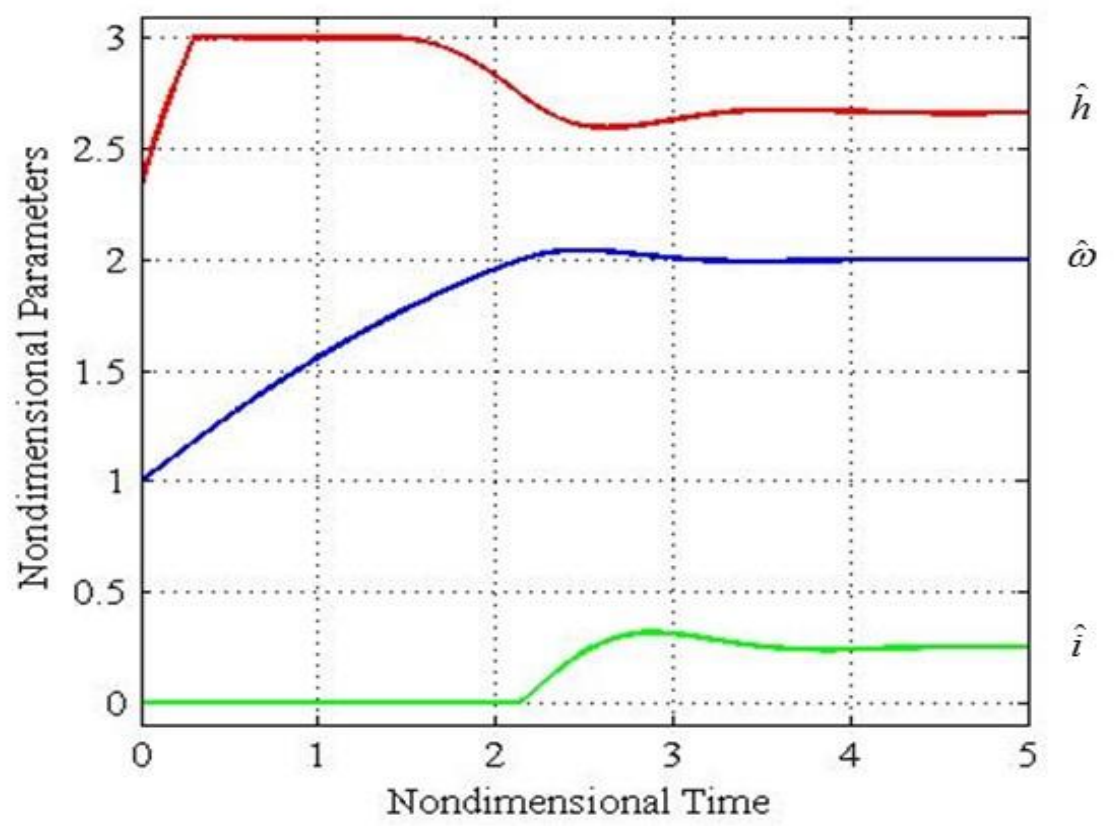

Figure 7-16. Transient response illustrating high speed control for $\hat{T}_{L}=1$ and $\hat{\omega}_{d}=2$. The blue line is shaft speed, the red line is MR fluid thickness, and the green line is current.

The initial shaft speed is given by $\hat{\omega}=1$. In order to increase the shaft speed, the controller seeks to provide a larger MR fluid thickness to reduce the non-energized brake torque. The initial error in the control signal is so large that the linear actuator soon saturates at the point where $\hat{h}=3$, which is the maximum allowable thickness that can be provided by the linear actuator due to a limited actuator stroke. While the fluid thickness remains saturated, the current in the electric coil is also saturated at zero which ensures that no energized torque is applied to the brake. During these minimal braking conditions, the shaft speed increases to the desired level $\hat{\omega}_{d}=2$. Once the desire speed is reached, the fluid thickness and the electric current become unsaturated and adjust themselves to create stronger braking conditions in order to hold the desired shaft speed. 
As shown in Fig. 7-16 the system is stable and the settling time occurs within 4 time constants. The transient response for speed exhibits very little overshoot. It is also significant to note that this high-speed condition could not have been achieved without the ability to control the fluid thickness $\hat{h}$.

\subsubsection{Moderate Speed Control}

Figure 7-17 represents a transient response for the controlled brake system where the applied load torque is given by $\hat{T}_{L}=1$ and the desired shaft speed is given by $\hat{\omega}_{d}=1$.

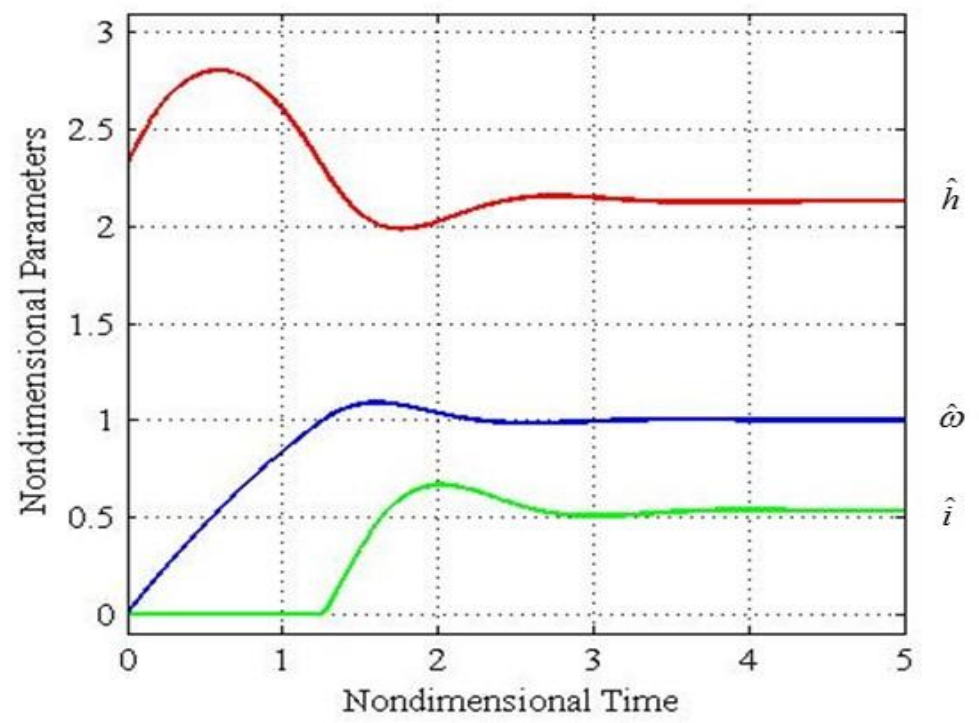

Figure 7-17. Transient response illustrating moderate speed control with a low-speed initial condition, and where $\hat{T}_{L}=1$ and $\hat{\omega}_{d}=1$. The blue line is shaft speed, the red line is MR fluid thickness, and the green line is current.

The initial shaft speed is given by $\hat{\omega}=0$. In order to increase the shaft speed, the controller seeks to provide a larger MR fluid thickness to reduce the non-energized brake torque. Since the desired speed is moderate, no saturation occurs in the linear actuator. During the initial increase of speed, the current in the electric coil is saturated at zero to 
ensure that no energized torque is applied to the brake. During these minimal braking conditions, the shaft speed increases to the desired level $\hat{\omega}_{d}=1$. Once the desire speed is reached, the fluid thickness and the electric current adjust themselves to create stronger braking conditions in order to hold the desired shaft speed. As shown in Fig. 7-17 the system is stable and the settling time occurs within 4 time constants. The response shown in Fig. 7-17 is very similar to that of Fig. 7-16 where the transient response for speed exhibits very little overshoot.

Figure 7-18 represents a transient response for the controlled brake system where the applied load torque is given by $\hat{T}_{L}=1$ and the desired shaft speed is given by $\hat{\omega}_{d}=1$;

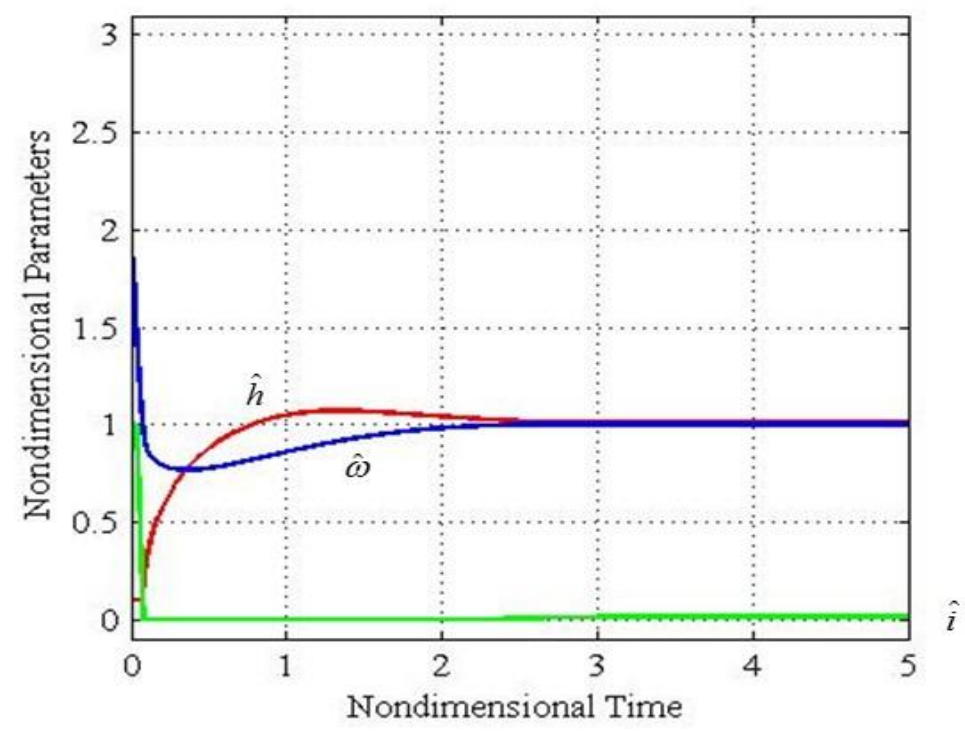

Figure 7-18. Transient response illustrating moderate speed control with a high-speed initial condition, and where $\hat{T}_{L}=1$ and $\hat{\omega}_{d}=1$. The blue line is shaft speed, the red line is MR fluid thickness, and the green line is current.

however, the initial shaft speed is now given by $\hat{\omega}=2$. This condition describes a control objective that is identical to that of the previous example, but the initial shaft speed is now greater than the desired shaft speed. In other words, the controller needs to make 
initial adjustments to slow the shaft down rather than speed the shaft up. In order to slow the shaft down, the controller starts with a small, saturated MR fluid thickness to increase the non-energized brake torque. At the same time, the electric current is saturated at its maximum level in order to apply a maximum energized brake torque. With both controllers applying a maximum brake torque to the shaft, the shaft speed drops quickly. Once the shaft speed approaches its desired value, the fluid-thickness control relaxes to its nominal operating condition and the magnetic brake de-energizes. Like the previous two examples, this response is stable and timely with a settling time of near 4 time constants. The speed response exhibits a bit more overshoot (undershoot) compared to the previous examples.

The important thing to note about the responses shown in Fig.s 7-17 and 7-18 is that while the control objective is the same for both examples, the steady-state solutions for $\hat{h}$ and $\hat{i}$ are different. This non-unique solution was discussed relative to Eq. (6.26) and has now been demonstrated with the same controller using different initial conditions. At this point one might question which of the two solutions is more advantageous from, say, an energy point of view. In order to consider this question, a model for the energy consumption of the linear actuator and the electric coil would need to be evaluated. Although not pursued here, based upon these energy models an optimal control solution may be pursued.

\subsubsection{Low Speed Control}

Figure 7-19 represents a transient response for the controlled brake system where the applied load torque is given by $\hat{T}_{L}=1$ and the desired shaft speed is given by $\hat{\omega}_{d}=0$. 


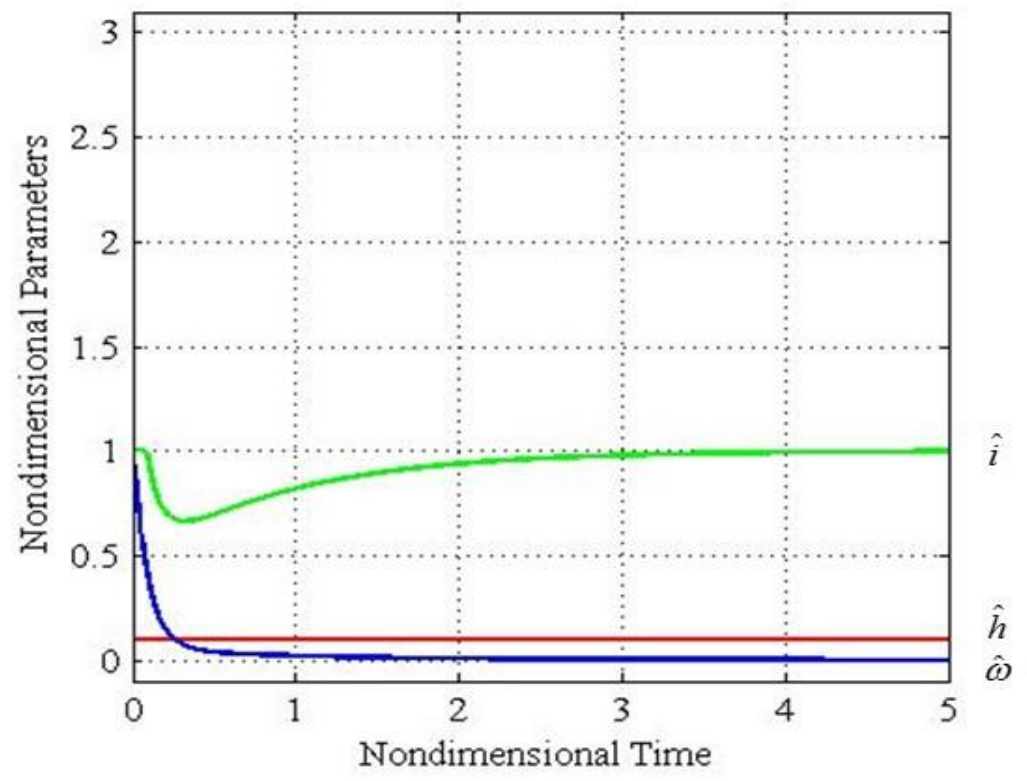

Figure 7-19. Transient response illustrating low speed control for $\hat{T}_{L}=1$ and $\hat{\omega}_{d}=0$. The blue line is shaft speed, the red line is MR fluid thickness, and the green line is current.

The initial shaft speed is given by $\hat{\omega}=1$. In order to decrease the shaft speed, the controller seeks to provide the smallest MR fluid thickness possible in order to apply the maximum non-energized brake torque. As such, the fluid thickness saturates at its minimum value $\hat{h}=0.1$. It is important to note that the non-energized brake torque is insufficient for stopping the shaft since the development of this torque requires a certain amount of shaft speed. In order to stop the shaft, the electric coil must be energized to its maximum value $\hat{i}=1$. As shown in Fig. 7-19 the maximum current is developed after 4 time constants at which point the desired shaft speed of zero has been achieved. Again, the response for the shaft speed is very timely and exhibits no overshoot (undershoot).

\subsubsection{Tracking Control}

Figure 7-20 represents a tracking response for the controlled brake system where 
the applied load torque is given by $\hat{T}_{L}=1$ and the desired shaft speed is given by $\hat{\omega}_{d}=1+0.2 \sin (2 \pi \hat{t})$. This desired shaft speed was arbitrarily selected to illustrate the tracking behavior of the control system.

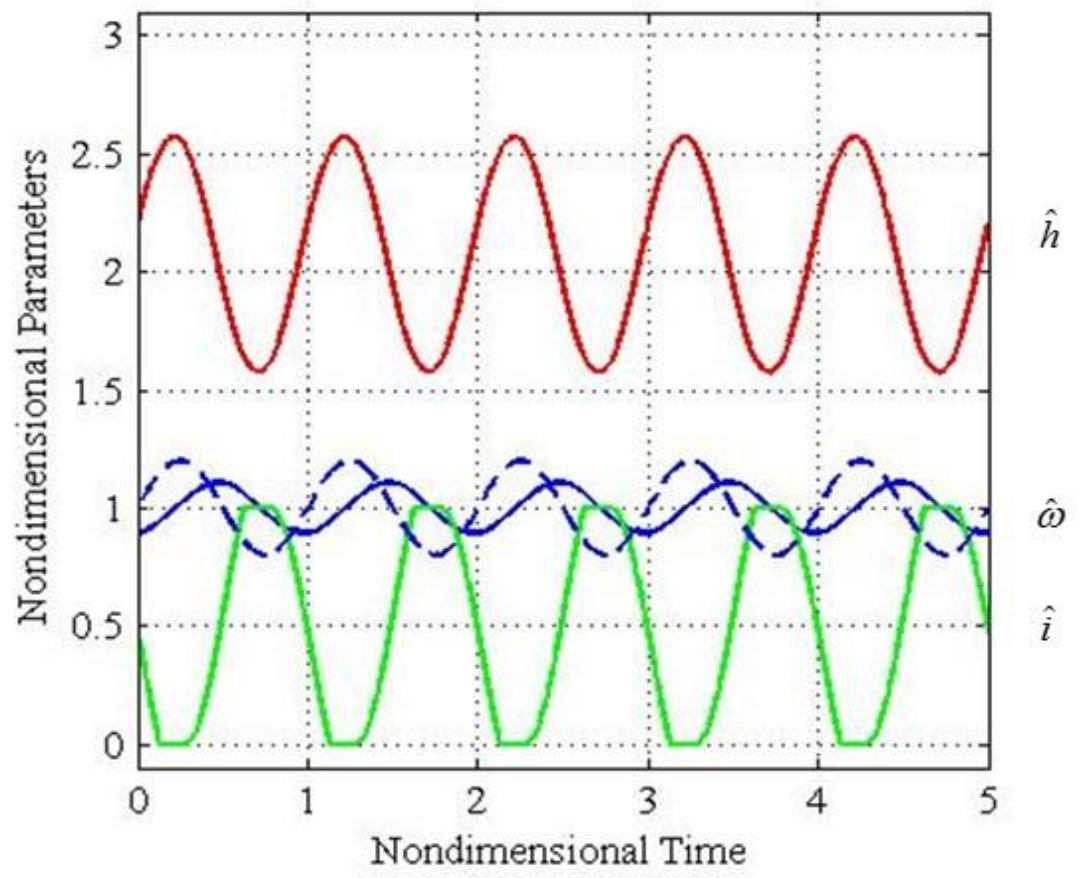

Figure 7-20. Tracking response illustrating moderate, sinusoidal speed control for $\hat{T}_{L}=1$ and $\hat{\omega}_{d}=1+0.2 \sin (2 \pi \hat{t})$. The dashed blue line is the desired speed while the solid blue line is actual speed, the red line is MR fluid thickness, and the green line is current.

As shown in this response, both the fluid thickness and the electric current are oscillating in order to track the desired shaft speed. The fluid thickness oscillates without saturation, while the electric current saturates at both maximum and minimum values. As shown in Fig. 7-20 the system demonstrates good stability; however, the desired tracking frequency clearly exceeds the bandwidth frequency of the system with an amplitude ratio of 0.5 and a phase lag of 90 degrees. 


\subsection{Summary}

In this chapter, results and discussion for frictional drum brake and MRF brake are presented. Variations in sensitivity coefficients for changes in nondimensional parameters for the frictional drum brake are presented and discussed first. Next, variations in sensitivity coefficients for changes in nondimensional parameters for the MRF brake for both geometrical and magnetic circuit designs are presented. Next, transient response illustrating low, moderate, and high speed control for different initial conditions are explained for a typical MRF brake. Next, tracking response illustrating moderate, sinusoidal speed control for tracking a desired speed is presented and discussed as well. Conclusions and recommendation for future work will be presented in the next chapter. 


\section{CHAPTER 8. CONCLUSIONS AND FUTURE WORK}

\subsection{General}

The previous chapters of the dissertation illustrate the mathematical modeling of both conventional frictional and MR fluid brake and sensitivity analyses. Based on some results of sensitivity analysis, new MR fluid brake designs considering increasing the braking torque is presented. Finally, controlling the MR fluid brake by controlling the applied current and fluid thickness is studied in detail.

\subsection{Conclusion}

The conclusion in this chapter is divided into three parts, sensitivity studies, suggested design, and control.

\subsubsection{Sensitivity Analysis}

The sensitivity analyses are carried out for both conventional and MR fluid brake and the following conclusions are supported by the analysis, results, and discussion as:

\subsubsection{Conventional Drum Brakes}

The following conclusions are supported by the previous chapters of this dissertation:

1. That the contact pressure and braking torque for the drum brake shown in Fig. 3-1 are dependent upon four nondimensional parameters of the brake: $\hat{a}, \hat{b}, \hat{c}$, and $\gamma$. See Eq. (3.12). 
2. That the braking torque is most sensitive to adjustments in $\hat{a}$ and $\hat{b}$, and that when seeking to increasing the braking torque of the design these parameters should be changed long before the design engineer seeks to alter $\hat{c}$ and $\gamma$. In order to increase braking torque $\hat{a}$ should be increased and $\hat{b}$ should be decreased.

3. That by altering $\hat{a}$ and $\hat{b}$ the contact pressure between the drum and the brake pad is altered, and that this alteration needs to be made carefully so as not to exceed the yield strength of the brake pad material. If the contact pressure exceeds the yield strength of the brake pad material, a mechanical failure of the brake will occur.

4. That the conclusions are quite general and robust, as demonstrated by the relatively flat lines in Figs. 7-1 through 7-3, for any drum brake design that is similar to the design shown in Fig. 3-1.

In summary, the results are useful for engineering design work that is aimed at increasing the braking torque for the drum brake shown in Fig. 3-1. The sensitivity analysis has identified the key parameters that must be adjusted in order to increase this torque, while concurrently identifying those parameter adjustments that are less impactful.

\subsubsection{MR fluid Brakes}

The following conclusions are supported by the previous chapters of this dissertation:

1. That both plastic and magnetic braking torques for a typical MRF brake shown are dependent upon seven nondimensional parameters of the brake: $\hat{w}, \hat{h}, \hat{\mu}, \hat{r}_{i}$ $, \hat{k}, \hat{H}$, and $\hat{\beta}$. See Eq. s (3.61) and (3.62). 
2. That the braking torque is most sensitive to adjustments in $w, h$, and $\mu$ for plastic braking torque and $k, H$, and $\beta$ for magnetic braking field. When seeking to increasing the braking torque of the design these parameters should be changed long before the design engineer seeks to alter $r_{o}$ and $r_{i}$. In order to increase braking torque, $\mu, k, H, r_{o}$, and $\beta$ should be increased and $h$ and $\hat{r}_{i}$ should be decreased.

3. That the conclusions are quite general and robust, as demonstrated by the relatively flat lines in Fig. s (7.4) through (7.9), for any MR fluid brake design that is similar to the typical MRF brake design.

4. Nondimensional braking torque is not sensitive for increasing $\hat{r}_{i}$ until $\hat{r}_{i}=0.6$.

However, it is dramatically decreases beyond this value.

In summary, the results of sensitivity analysis are useful for engineering design work that is aimed at increasing the braking torque for the typical MRF brake. The sensitivity analysis has identified the key parameters that must be adjusted in order to increase this torque, while concurrently identifying those parameter adjustments that are less impactful.

\subsection{Controlling the MR fluid brake}

The following conclusions are supported by the analysis, results, and discussion of the controlling the MR fluid brake chapter as follows:

1. The MR fluid brake is capable of generating torque from two sources: 1) a nonenergized source resulting from the Newtonian viscosity, angular velocity, and MR fluid thickness; and 2) an energized source resulting from the MR fluid which increase its viscosity when exposed to a magnetic field. The non-energized brake 
torque is controlled by altering the MR fluid thickness. The energized brake torque is controlled by altering the current passing through the electric coil, which then alters the strength of the magnetic field.

2. By simultaneously controlling the MR fluid thickness and the electric current, a large range of brake torque is achieved without increasing the radial envelop for the brake, and saturation conditions in one controller are compensated for by the other controller. High angular velocities of the brake are primarily controlled by increasing the MR fluid thickness, while low angular velocities are primarily controlled by increasing the electric current.

3. By enforcing the design constraint, the strength of the non-energized torque and the energized torque can be made comparable. The ability to satisfy this design constraint has been illustrated in the Appendix.

4. The governing equation for describing the angular velocity of the MR fluid brake can be made quite elegant using nondimensional analysis. The physics of this problem are captured by three things: 1) the applied nondimensional load-torque, 2) the nondimensional MR fluid thickness, and 3) the nondimensional electric current. An alteration in any one of these three things will impact the dynamic and steady-state response of the brake.

5. Two PI controls have been used to simultaneously adjust the MR fluid thickness and the electric current. These linear controllers have been selected because they are known to produce an easily tuned response with zero steady-state error. These linear controllers have been shown to perform well for controlling the described nonlinear 
system. Good transient responses for regulating a constant speed (high, moderate, and low), and good stability while seeking to track a sinusoidal input have been achieved.

6. Because both the fluid thickness and the electric current are being simultaneously controlled to achieve a single speed objective, a unique solution does not exist for the combination of fluid thickness and current that will achieve our goal. Rather, the combination of fluid thickness and electric current is dependent upon the initial conditions, and whether or not any saturation has been experienced along the way. In the end, however, the control objective is always achieved in a timely and stable way. In summary, the proposed control system for the MR fluid brake has demonstrated good controllability for the MR fluid brake. The nondimensional form of this analysis has been used to generalize our results for any design that is similar to the system shown in Fig. 6-5. It is important to note that all nondimensional solutions may be redimensionalized for using in the practical engineering applications.

8.4 The Torque Capacity of a Magnetorheological Fluid Brake Compared To a Frictional Disk Brake

The following conclusions are supported by the analysis and comparisons that have been presented in Appendix:

1. Only three-dimensional parameters are needed to compare the torque capacity of a frictional brake with the torque capacity of the MR fluid brake: the maximum allowable contact pressure and the coefficient-of-friction for the frictional lining, and the yield shear-stress for the MR fluid. 
2. For brakes of similar size, the frictional brake is able to generate 10 to 18 times more torque than the MR fluid brake.

3. For brakes with a similar torque capacity, the outer radius of the MR fluid brake must be twice as large as the outer radius of the frictional brake.

These conclusions provide useful guidelines for the engineer who is considering the relative merits of using a MR fluid brake as opposed to a frictional disk brake.

\subsection{Recommendation for the Future Work}

In the current work, two configurations of a Magnetorheological (MR) fluid brake as a choice for substitution to the conventional frictional brake are presented. The new designs have several advantages compared to the conventional brake that makes it easier for design and control. Some recommendations can be considered and encouraged for the future work as follows:

1- Achieving an experimental work to validate the performance of the new suggested designs and get a feedback about the real behavior of the MR fluid brake under practical working conditions.

2- Try to optimize MR fluid properties by considering fluid synthetization processes. For example, increase MR fluid shear stress (on field shear stress) by adding some chemical components.

3- Study effect of magnetic gab on the magnetic force created through out like chain structures of the MR fluid in the microscale instead of macroscale. 


\section{REFERERENCES}

[1]. Http://www.dbrake.com/braking-history.php.

[2]. Https://encryptedtbn1.gstatic.com/images.

[3]. Delphi Co., Delphi Brake Modules - Electric Caliper, Troy, MI, 2005].

[4]. K. Lee and K. H. Park,” Optimal Robust Control of a Contactless Brake System Using an Eddy Current", Mechatronics, 4, p615-631, 1999.

[5]. K. Lee and Kyi H. Park," Modeling Eddy Currents with Boundary Conditions by Using Coulomb's Law and the Method of Images”, IEEE Transactions on Magnetics, 38(2), p1333-1340, March 2002.

[6]. E. Gedik, H. Kurt, Z. Recebli, and C. Balan, "Two-dimensional CFD simulation of magnetorheological fluid between two fixed parallel plates applied external magnetic field," Comput. Fluids, vol. 63, pp. 128-134, Jun. 2012.

[7]. S. Melle and J. E. Martin, "Chain model of a magnetorheological suspension in a rotating field,” J. Chem. Phys., vol. 118, no. 21, pp. 9875-9881, 2003.

[8]. Bigué, J. P. L., Charron, F., \&Plante, J. S. (2015). Understanding the super-strong behavior of magnetorheological fluid in simultaneous squeeze-shear with the Péclet number. Journal of Intelligent Material Systems and Structures, $1045389 X 15577657$.

[9]. G. Bossis, S. Lacis, A. Meunier, and O. Volkova, "Magnetorheological fluids," J. Magn. Magn.Mater., vol. 252, pp. 224-228, 2002.

[10]. Goncalves, F., "Dynamic Analysis of Semiactive Control Techniques for Vehicle Applications", Master of Science Thesis, Department of Mechanical Engineering, 
Virginia Tech, August 2001.

[11]. Linder, J.E., Dimcock, D.A., and Wereley, N.M., "Design of a Magnetorheological Automotive Shock Absorber", Proceedings of SPIE, volume 3985, 2000, pp. 426437.

[12]. Gordaninejad, F., Kelso, S.P., "Magneto-Rheological Fluid Shock Absorbers for HMMWV".Proceedings of SPIE, volume 3989, 2000, pp. 266-273.

[13]. Peel, D.J., Stanway, R., Bullough, W.A., "Design Optimisation of a Controllable Vibration Damper for Vehicle Suspension Applications", Active Control of Vibration and Noise, American Society of Mechanical Engineers, Design Engineering Division Publication, DE-Volume 93, 1996, pp. 205-214.

[14]. B. Liu, W.H. Li, P. B. Kosasih and X. Z. Zhang,” Development of an MRbrakebased haptic device”, Smart Materials and Structures, 15, p1960-1966, 2006.

[15]. B.M. Kavlicoglu, F. Gordaninejad, C.A. Evrensel, N. Cobanoglu, Y. Liu, A. Fuchs and G. Korol,” A high-torque magnetorheological fluid clutch”, Proceed- ings of SPIE Conference on Smart Materials and Structures, San Diego, CA, USA, March 2002.

[16]. S. Gopalswamy, S. M. Linzell and G. L. Jones, Magnetorheological fluid clutch with minimized reluctance, US Patent 5,845,752, United States Patent Office, December 81998.

[17]. Carlson, J.D., Matthis, W., and Toscano J.R., "Smart Prosthetics Based On Magnetorheological Fluids", SPIE 8th Annual Symposium on Smart Structures and Materials, Newport Beach, CA, March 2001.

[18]. Tang, X., Zhang, X., \& Tao, R. (1999). Flexible fixture device with magneto- 
rheological fluids. Journal of intelligent material systems and structures,10(9), 690694].

[19]. Manring, N. D., \& Mehta, V. S. (2011). Physical limitations for the bandwidth frequency of a pressure controlled, axial-piston pump. Journal of Dynamic Systems, Measurement, and Control, 133(6), 061005.

[20]. Manring, N. D. (2003, January).Sensitivity analysis of the conical-shaped equivalent model of a bolted joint. In ASME 2003 International Mechanical Engineering Congress and Exposition (pp. 435-439). American Society of Mechanical Engineers.

[21]. David J. Pannell . Sensitivity analysis: strategies, methods, concepts, examples. Full Paper- David Pannell's homepage- The University of Western Australia, Crawley 6009, Australia, viewed August 20, 2015.

[22]. Ostermeyer, G. P. (2001, February). Friction and wear of brake system. ForschungimIngenieurwesen, 66(6), 267.272.

[23]. Othaganont, P., Assadian, F., \& Auger, D. (2014).Sensitivity Analyses for CrossCoupled Parameters in Automotive Powertrain Optimization. Energies,7(6), 37333747.

[24]. Tortorelli, D. A., \&Michaleris, P. (1994). Design sensitivity analysis: overview and review. Inverse problems in Engineering, 1(1), 71-105.

[25]. Kucherenko, S., Feil, B., Shah, N., \&Mauntz, W. (2011).The identification of model effective dimensions using global sensitivity analysis. Reliability Engineering \& System Safety, 96(4), 440-449.

[26]. Hwang, I. J., \& Park, G. J. (2008). Mode and design sensitivity analyses for brake 
judder reduction. Proceedings of the Institution of Mechanical Engineers, Part D: Journal of Automobile Engineering, 222(7), 1259-1272.

[27]. Anstett-Collin, F., Goffart, J., Mara, T., \& Denis-Vidal, L. (2015).Sensitivity analysis of complex models: coping with dynamic and static inputs. Reliability Engineering \& System Safety, 134, 268-275.

[28]. Martins, J. R. R. A., Kroo, I. M., \& Alonso, J. J. (2000).An automated method for sensitivity analysis using complex variables.AIAA paper, 689, 2000.

[29]. Yang, C. P., \& Liu, T. (2013, December).Analysis of a New Mechanical Anti-Lock Brake System for Two-Wheeled Vehicles.In Applied Mechanics and Materials (Vol. 437, pp. 313-320).

[30]. Rocha, A. B., \& Silveira, Z. C. (2011, June). Conceptual design of brake disc applied to hybrid electric vehicles. In the 13th World Congress in Mechanisms and Machine Science, Guanajuato, México, June 19-25, 2011.

[31]. Huang, J., Zhang, J. Q., Yang, Y., \& Wei, Y. Q. (2002). Analysis and design of a cylindrical magneto-rheological fluid brake. Journal of Materials Processing Technology, 129(1), 559-562.

[32]. Park, E. J., Stoikov, D., da Luz, L. F., \&Suleman, A. (2006). A performance evaluation of an automotive magnetorheological brake design with a sliding mode controller. Mechatronics, 16(7), 405-416.

[33]. Rossa, C., Jaegy, A., Micaelli, A., \&Lozada, J. (2014).Development of a multilayered wide-ranged torque magnetorheological brake. Smart Materials and Structures, 23(2), 025028.

[34]. Sohn, J. W., Jeon, J., Nguyen, Q. H., \& Choi, S. B. (2015). Optimal design of disc- 
type magneto-rheological brake for mid-sized motorcycle: experimental evaluation. Smart Materials and Structures, 24(8), 085009.

[35]. Bigué, J. P. L., Charron, F., \&Plante, J. S. (2015).Understanding the super-strong behavior of magnetorheological fluid in simultaneous squeeze-shear with the Péclet number. Journal of Intelligent Material Systems and Structures, $1045389 X 15577657$.

[36]. Shiao, Y., \& Nguyen, Q. A. (2014). Torque Enhancement for a New Magnetorheological Brake. Procedia Engineering, 76, 12-23.

[37]. Rossa, C., Jaegy, A., Lozada, J., \&Micaelli, A. (2014). Design considerations for magnetorheological brakes. Mechatronics, IEEE/ASME Transactions on,19(5), $1669-1680$.

[38]. Phu, D. X., Choi, S. B., Lee, Y. S., \& Han, M. S. (2014). Design of a new engine mount for vertical and horizontal vibration control using magnetorheological fluid. Smart Materials and Structures, 23(11), 117001.

[39]. Nguyen, Q. H., Nguyen, N. D., \& Choi, S. B. (2015). Design and evaluation of a novel magnetorheological brake with coils placed on the side housings. Smart Materials and Structures, 24(4), 047001.

[40]. Nguyen, Q. H., Lang, V. T., \& Choi, S. B. (2015). Optimal design and selection of magneto-rheological brake types based on braking torque and mass. Smart Materials and Structures, 24(6), 067001.

[41]. Russo, R., \&Terzo, M. (2011).Design of an adaptive control for a magnetorheological fluid brake with model parameters depending on temperature and speed. Smart Materials and Structures, 20(11), 115003. 
[42]. Nguyen, Q. H., \& Choi, S. B. (2012). Selection of magnetorheological brake types via optimal design considering maximum torque and constrained volume. Smart Materials and Structures, 21(1), 015012.

[43]. Park, E. J., da Luz, L. F., \&Suleman, A. (2008). Multidisciplinary design optimization of an automotive magnetorheological brake design. Computers \& structures, 86(3), 207-216.

[44]. Nguyen, Q. H., Lang, V. T., Nguyen, N. D., \& Choi, S. B. (2014). Geometric optimal design of a magneto-rheological brake considering different shapes for the brake envelope. Smart Materials and Structures, 23(1), 015020.

[45]. Song, B. K., Nguyen, Q. H., Choi, S. B., \& Woo, J. K. (2013). The impact of bobbin material and design on magnetorheological brake performance. Smart Materials and Structures, 22(10), 105030.

[46]. Nguyen, Q. H., \& Choi, S. B. (2010).Optimal design of an automotive magnetorheological brake considering geometric dimensions and zero-field friction heat. Smart Materials and Structures, 19(11), 115024.

[47]. Choi, S. B., Yook, J. Y., Choi, M. K., Nguyen, Q. H., Lee, Y. S., \& Han, M. S. (2007). Speed Control of DC Motor using Electrorheological Brake System. Journal of Intelligent Material Systems and Structures, 18(12), 1191-1196.

[48]. Nguyen, P. B., \& Choi, S. B. (2011). A new approach to magnetic circuit analysis and its application to the optimal design of a bi-directional magnetorheological brake. Smart Materials and Structures, 20(12), 125003.

[49]. Erol, O. G., Gurocak, H., \&Gonenc, B. (2011, January). Design optimization method for MR-brake actuators. In ASME 2011 International Mechanical 
Engineering Congress and Exposition (pp. 1257-1263). American Society of Mechanical Engineers.

[50]. Rossa, C., Eck, L., Micaelli, A., \&Lozada, J. (2014).On a novel torque detection technique for magnetorheological actuators. Sensors Journal, IEEE, 14(4), 12231231.

[51]. Assadsangabi, B., Daneshmand, F., Vahdati, N., Eghtesad, M., \&Bazargan-Lari, Y. (2011).Optimization and design of disk-type MR brakes. International Journal of Automotive Technology, 12(6), 921-932.

[52]. Polcar, P. (2012). Magnetorheological brake design and experimental verification. In 2012 ELEKTRO.

[53].Perniata, A. N. R. S., Tjahjana, D. D. D. P., Nizam, M., Mazlan, S. A., \&Imaduddin, F. (2014, November). Simulation and experimental studies on braking response of inertial load using magnetorheological brake. In Electrical Engineering and Computer Science (ICEECS), 2014 International Conference on (pp. 353-358). IEEE.

[54]. Ma, L., Yu, L., Liu, X., Wang, Z., \& Pan, N. (2014). Experimental Research and Optimal Design of an Automotive Magneto-Rheological Brake System (No. 201401-2534).SAE Technical Paper.

[55]. Sukhwani, V. K., \&Hirani, H. (2008).Design, development, and performance evaluation of high-speed magnetorheological brakes. Proceedings of the Institution of Mechanical Engineers, Part L: Journal of Materials Design and Applications, 222(1), 73-82.

[56]. Ma, L., Yu, L., Song, J., Xuan, W., \& Liu, X. (2015). Design, Testing and Analysis 
of a Novel Multiple-Disc Magnetorheological Braking Applied in Vehicles (No. 2015-01-0724). SAE Technical Paper.

[57]. Yang, L., Duan, F., \& Eriksson, A. (2008).Analysis of the optimal design strategy of a magnetorheological smart structure. Smart Materials and Structures, 17(1), 015047.

[58]. Jolly, M. R., Carlson, J. D., \& Munoz, B. C. (1996).A model of the behavior of magnetorheological materials. Smart Materials and Structures, 5(5), 607.

[59]. Wereley, N. M., \& Pang, L. (1998).Nondimensional analysis of semi-active electrorheological and magnetorheological dampers using approximate parallel plate models. Smart Materials and Structures, 7(5), 732.

[60]. Chen, S., Huang, J., Jian, K., \& Ding, J. (2015). Analysis of influence of temperature on magnetorheological fluid and transmission performance. Advances in Materials Science and Engineering, 2015.

[61]. Wang, D., Zi, B., Zeng, Y., Hou, Y., \&Meng, Q. (2014).Temperature-dependent material properties of the components of magnetorheological fluids. Journal of Materials Science, 49(24), 8459.8470.

[62]. Kim, M., \& Park, S. J. (2016). A comprehensive viscosity model for micro magnetic particle dispersed in silicone oil. Journal of Magnetism and Magnetic Materials, 404, 4044.

[63]. Patel, S. R., Powar, K. P., \&Sawant, S. M. (2016). Thermal analysis of magnetorheological brake for automotive application. Applied Thermal Engineering, 98, 238-245.

[64]. Li, W. H., \& Zhang, X. Z. (2008). The effect of friction on magnetorheological 
fluids. Korea - Australia Rheology Journal, vol. 20, no. 2, pp. `45-50.

[65]. Wang, D. M., Hou, Y. F., \&Tian, Z. Z. (2013). A novel high-torque magnetorheological brake with a water cooling method for heat dissipation. Smart Materials and Structures, 22(2), 025019.

[66]. Norton, R.L. 2014. Machine Design: An Integrated Approach, 5thed. Prentice Hall, New York.

[67]. Budynas, R.G., and J.K. Nisbett. 2008. Shigley’s Mechanical Engineering Design, 8thed. McGraw Hill Higher Education, New York.

[68]. Juvinall, R.C., and K.M. Marshek. 2006. Fundamentals of Machine Component Design, 4thed. John Wiley \& Sons, Inc., New York.

[69]. Spotts, M.F., T.E. Shoup, and L.E. Hornberger. 2004. Design of Machine Elements, 8thed. Pearson Education, Inc., Upper Saddle River, New Jersey.

[70]. Collins, J.A.2003. Mechanical Design of Machine Elements and Machines: A Failure Preventions Perspective. John Wiley \& Sons, Inc., New York.

[71. Dimarogonas, A.D.2001. Machine Design: A CAD Approach. John Wiley \& Sons, Inc., New York.

[72]. Mott, R.L. 1999. Mechanical Elements in Mechanical Design, 3rded. Prentice Hall, Upper Saddle River, New Jersey.

[73]. Sayim, I., and D. Zhang.2016. Optimization of the brake factor for an S-cam foundation brake using RSM. Journal of Mechanical Engineering, 62 (2016) 9, pp. 503-510. DOI:10.5545/sv-jme.2016.3459.

[74]. Huang, J., C.M. Krousgrill, and A.K. Bajaj.2006. Modeling of automotive drum brakes for squeal and parameter sensitivity analysis. Journal of Sound and 
Vibration, 289 (2006), pp. 245-63.

[75]. Nechak, L., F. Gillot, S. Besset, and J.J. Sinou. 2015. Sensitivity analysis and Kriging based models for robust stability analysis of brake systems. Mechanics Research Communications, 69 (2015), pp. 136-145.

[76]. Gui, L., X. Wang, Z. Fan, and F. Zhang. 2016. A simulation method of thermosmechanical and tribological coupled analysis in dry sliding systems. Tribology International, 103 (2016), pp. 121-131.

[77]. Liu, N., Z. Zheng, Y. Wu, X. Kong, and H. Ding. 2015. Finite element analysis of drum brake assembly. Chemical Engineering Transactions, Vol 46, 2015, pp. 1057-1062. DOI:103303/CET1546177

[78]. Hohmann, C., K. Schiffner, K. Oerter, and H. Reese. 1999. Contact analysis for drum brakes and disk brakes using ADINA. Computers and Structures, 72 (1999), pp. 185-198.

[79]. Mariola Rodríguez and FundaciónFatronik (2008). Completely flexible and reconfigurable fixturing of complex shaped workpieces with MRF. 6th FPPeriodic Activity Report no. PAR_2 Final Activity Rep: v1.

[80] Https://en.wikipedia.org/wiki/Magnetorheological_fluid.

[81]. A. G. Olabi and A. Grunwald, Design and Application of Magneto-Rheological Fluid, Materials and Design, Vol. 28, No. 10, 2007, 2658-2664.

[82]. J. D. Carlson and M. R. Jolly, MR Fluid, Foam and Elastomer Devices, Mechatronics, Vol. 10, Nos. 4-5, 2000, 555-569.

[83]. T. Butz and O. von Stryk, Modelling and Simulation of Electro-and 
Magnetorheological Fluid Dampers, Zeitschrift Fur Angewandte Mathematik Und Mechanik, Vol. 82, 2002, 3-20.

[84]. J. -H. Yoo and N. M. Wereley, Design of a High-Efficiency Magnetorheological Valve, Journal of Intelligent Material Systems and Structures, Vol. 13, 2002, 679685.

[85]. M Y. Tian and Q. Zou, Normalized Method for Comparing Tensile Behaviors of Electrorheological Fluids, Applied Physics Letters, Vol. 82, No. 26, 2003, 48364838.

[86]. M P. Kulkarni, C. Ciocanel, S. L. Vieira and N. Naganathan, Study of the Behavior of MR Fluids in Squeeze, Torsional and Valve Modes, Journal of Intelligent Material Systems and Structures, Vol. 14, 2003, 99-104.

[87]. M J. -H. Yoo, J. Sirohi and N. M. Wereley, A Magnetorheological Piezohydraulic Actuator, Journal of Intelligent Material Systems and Structures, Vol. 16, 2005, 645-953.

[88]. M E. Germer, Magnetic Valve, US Patent 2670749, 1954.

[89]. M S. P. Kelso, Experimental Characterization of Commercially Practical Magnetorheological Fluid Damper Technology, in proceedings of the SPEE Conference on Smart Structures and Materials, Newport Beach, CA, 2001, 43324339.

[90]. M P. L. Wong, W. A. Bullough, C. Feng and S. Lingard, Tribological Performance of a Magneto-Rheological Suspension, Wear, Vol. 247, No. 1, 2001, 33-40.

[91]. M G. J. Monkman, The Electrorheological Effect under Compressive Stress, Journal Physics D: Applied Physics, Vol. 28, 1995, 588-593. 
[92]. M K. -J. Kim and C. -W. Lee, Design and Modeling of Semi-Active Squeeze Film Damper Using Magnetorheological Fluid, in proceedings of the 7th International Conference on Motion and Vibration Control (MOVIC 2004), Saint Louis, USA, 2004, 1-11.

[93]. M C. R. Burrows, N. C. Kucuk, M. N. Sahinkaya and R. Stanway, Linearized Squeeze-Film Dynamics: A Model Structure and the Interpretation of Experimentally Derived Parameters, in proceedings of the Institution of Mechanical Engineers, Part C, Journal of Mechanical Engineering Science, Vol. 204, No. 4, 1990, 263-272.

[94]. M J. L. Sproston, S. G. Rigby, E. W. Williams and R. Stanway, A Numerical Simulation of Electrorheological Fluids in Oscillatory Compressive Squeeze Flow, Journal of Physics D: Applied Physics, Vol. 27, 1994, 338-343.

[95]. M J. L. Sproston, A. K. El Wahed and R. Stanway, Electrorheological Fluids in Dynamic Squeeze Flow, in proceedings of the IEEE International Conference on Conduction \& Breakdown in Dielectric Liquids, ICDL, Roma, Italy, 1996, 515 519.

[96]. S. B. Choi, H. J. Choi, Y. T. Choi and N. M. Wereley, Preparation and Mechanical Characteristics of Poly(methylaniline) Based Electrorheological Fluid, Journal of Applied Polymer Science, Vol. 96, 2005, 1924-1929.

[97]. Y. Tian, M. Zhang, Y. Meng and S. Wen, Transient Response of Compressed Electrorheological Fluid, Journal of Colloid and Interface Science, Vol. 290, No. 1, 2005, 289-297.

[98]. H. See, S. Mackenzie and B. T. Chua, Effect of Compression on the Response of a 
Magneto-Rheological Suspension, Korea-Australia Rheology Journal, Vol. 18, No. 3, 2006, 121-126.

[99]. Y. Tian, Y. Meng, H. Mao and S. Wen, Electrorheological Fluid Under Elongation, Compression, and Shearing, Physical Review E, Vol. 65, No. 3, 2002, 031507.

[100]. A. Lukkarinen and K. Kaski, Simulation Studies of Electrorheological Fluids under Shear, Compression, and Elongation Loading, Journal of Applied Physics, Vol. 83, 1998, 1717-1725.

[101]. Avraam, M. T. (2009). MR- fluid brake design and its application to a portable muscular rehabilitation device. Doktora Tezi, Université Libre De Bruxelles, Faculté De Sciences Appliquées.

[102]. J. F. Douglas, J. M. Gasiorek and J. A. Swaffield, Fluid Mechanics, 3rd Ed., Longman Scientific \& Technical, Harlow Essex, UK, 1995.

[103]. [118] J. M. Gere and S. P. Timonshenko, Mechanics of Materials, 3rd Ed., PWSKENT Publishing Company, Boston, 1990.

[104]. Karakoc, K. (2007). Design of a magnetorheological brake system based on magnetic circuit optimization (Doctoral dissertation, University of Victoria).

[105]. W. D. Callister Jr., Materials Science and Engineering: An Introduction, 3rd Ed., John Wiley and Sons, Inc., New York, 1994.

[106]. D. R. Askeland, The Science and Engineering of Materials, 3rdEd., Chapman and Hall, London, 1996.

[107]. Erickson, R. W., \& Maksimovic, D. (2007). Fundamentals of power electronics. Springer Science \& Business Media.

[108]. Gogue, G., \& Stupak Jr, J. (1993). Theory and practice of electromagnetic design 
of DC motors and actuators. Oregon, USA: G2 Consulting.

[109]. Box, G., Bisgaard, S., \& Fung, C. (1988). An explanation and critique of Taguchi's contributions to quality engineering. Quality and reliability engineering international, 4(2), 123-131.Chicago

[110]. Roy, R. K. (2010). A primer on the Taguchi method. Society of Manufacturing Engineers.

[111]. Taguchi, G., \& Clausing, D. (1990). Robust quality. Harvard Business Review,68(1), 65-75.

[112]. Box, G. E., Hunter, J. S., \& Hunter, W. G. (2005). Statistics for experimenters: design, innovation, and discovery (Vol. 2). New York: Wiley-Interscience.

[113]. Montgomery, D. C. (1991). Design and analysis of experiments.

[114]. Dale, B. G., Van Der Wiele, T., \& Van Iwaarden, J. (2013). Managing quality. John Wiley \& Sons.

[115]. Summers, D. C. (2005). Quality management: Creating and sustaining organizational effectiveness (p. 409). Pearson Prentice Hall.

[116]. Tanco, M., Viles, E., Ilzarbe, L., \& Álvarez, M. J. (2007, July). Manufacturing industries need Design of Experiments (DoE). In World Congress on Engineering (pp. 1108-1112).

[117]. Montgomery, D. C., \& Runger, G. C. (2010). Applied statistics and probability for engineers. John Wiley \& Sons.

[118]. Popat, M. A. (2011). An investigation and analysis of process parameters for EDM drilling machine using Taguchi method.

[119]. Https://onlinecourses.science.psu.edu/stat503/node/67. 
[120]. Phadke, S. M. (1989). Quality Engineering Using Robust Design Prentice Hall Englewood Cliffs. NJ Google Scholar.

[121]. Taguchi, G., Chowdhury, S., \& Taguchi, S. (2000). Robust engineering. McGrawHill Professional.

[122]. Phadke, M. S. Quality engineering using robust design, 1989. Englewood Cliffs, New Jersey: PTR Prentice-Hall Inc.

[123]. Ross, P. J. (1988). Taguchi techniques for quality engineering: loss function, orthogonal experiments, parameter and tolerance design.

[124]. Hedayat, A. S., Sloane, N. J. A., \& Stufken, J. (2012). Orthogonal arrays: theory and applications. Springer Science \& Business Media.

[125]. Gunter, B. (1987). A perspective on the Taguchi methods. Quality Progress,20(6), 44-52.

[126]. R. K. (2010). A primer on the Taguchi method. Society of Manufacturing Engineers.

[127]. Taguchi, G., Chowdhury, S., \& Taguchi, S. (2000). Robust engineering. McGrawHill.

[128]. Ross, P.J. (1988), “Taguchi techniques for quality engineering”, McGrawHillBook Company, New York.

[129]. Roy, R.K. (1990), “A primer on Taguchi method”, Van Nostrand Reinhold, New York.

[130]. 10. Barker, T.B. (1990), "Engineering quality by design”, Marcel Dekker, Inc., New York.

[131]. Ross, P.J. (1988), “Taguchi techniques for quality engineering”, McGraw- 
HillBook Company, New York.

[132]. Logothetis, N. (1994), Managing for Total Quality, Prentice -Hall, Englewood Cliffs, NJ]

[133]. Zhang H H, Liao C R, Chen W M and Huang S L 2006. A magnetic design method of MR fluid dampers and FEM analysis on magnetic saturation J. Intell. Mater. Syst. Struct.17 813-8

[134]. Ginder JM and Davis LC (1994) Shear stresses in magnetorheological fluids: role of magnetic saturation. Applied Physics Letters 65(26): 3410-3412.

[135]. N. Guo, H. Du, and W. Li, "Finite element analysis and simulation evaluation of a magnetorheological valve,” Int. J. Adv. Manuf. Technol., vol. 21, pp. 438-445, 2003.

[136]. R. Sahoo, M.V. Rasna, D. Lisjak, A. Mertelj, S. Dhara, Appl. Phys. Lett. 106 (2015) 161905.

[137]. Gravatt, J. W. (2003). Magneto-rheological dampers for super-sport motorcycle applications (Doctoral dissertation, Virginia Polytechnic Institute and State University).

[138]. Thesis, James. Poynor, J. C. (2001). Innovative designs for magneto-rheological dampers (Doctoral dissertation, Virginia Tech).

[139]. Thesis MR. Goncalves, F. D. (2005). Characterizing the behavior of magnetorheological fluids at high velocities and high shear rates (Doctoral dissertation, Virginia Tech).

[140]. Lord Rheonetic Magnetically Responsive Technology, Hydrocarbon-Based MR 
Fluid MRF-132LD, Product Bulletin, 2002.

[141]. Https:/www.ndeed.org/EducationResources/ Community College/magParticle/ Hysteresis Loop.Htm (Advanced Technological Educatiom) program through grant due 0101709 .

[142]. J. D. Kraus, Electromagnetics, International Edition, 4th142J. D. Kraus, Electromagnetics, International Edition, 4th Edition, Mc-Graw-Hill Inc., Singapore, 1991.

[143] Bhatnagar, R. (2013). Modeling and design of a dual channel magnetorheological damper.

[144] Cvek, M. (2014). Magnetorheological properties of the suspensions based on modified magnetic filler.

[145] Nassar, W. (2012). Pre-yield shearing regime of a magnetorheological fluid (MRF) (Doctoral dissertation, Ecole Polytechnique X).

[146]. G. Song and M. Zeng, A Thin-Film Magnetorheological Fluid Damper/Lock, Smart Materials and Structures, Vol. 14, 2005, 369-375.

[147]. J. H. Park, M. H. Kwon and O. O. Park, Rheological Properties and Stability of Magnetorheological Fluids using Visco-elastic Medium and Nanoadditives, Korean Journal of Chemical Engineering, Vol. 18, No. 5, 2001, 580-585.

[148]. H. Bose and A. Trendler, Smart Fluids - Properties and Benefit for New Electromechanical Devices, AMAS Workshop on Smart Materials and Structures, SMART'03, Jadwisin, 2003, 329-336.

[149]. Https://en.wikipedia.org/wiki/Coercivity.

[150]. J. H. Park and O. O. Park, Electrorheology and Magnetorheology, K Australia 
Journal, Vol. 13, No. 1, 2001, 13-17.

[151]. B. C. Munoz, G. W. Adams, V. T. Ngo and J. R. Kitchin, Stable Magnetorheological Fluids, US Patent 6203717, 2001.

[152]. C. Fang, B. Y. Zhao, L. S. Chen, Q. Wu, N. Liu and K. A. Hu, The Effect of the Green Additive Guar Gum on the Properties of Magnetorheological Fluid, Smart Materials and Structures, Vol. 14, No. 1, 2005, N1-N5.

[153]. J. Rabinow, The Magnetic Fluid Clutch, Transactions of the AIEE, Vol. 67, 1948, 1308-1315.

[154]. J. Rabinow, Magnetic Fluid Torque and Force Transmitting Device, US Patent $2575360,1951$.

[155]. Li, W.H., and H. Du. 2003. Design and experimental evaluation of magnetorheological brake. The International Journal of Advanced Manufacturing Technology. 21:508-515, pp. 508-15.

[156]. Carlson, A.B., and D.G. Gisser. 1990. Electrical Engineering Concepts and Applications, 2nd ed. Addison-Wesley Publishing Company, New York.

[157]. LORD Technical Data. Accessed 2017. MRF-132DG Magneto-Rheological Fluid. https://www.lord.com/sites/default/files/Documents/TechnicalDataSheet/DS7015 MRF-132DGMRFluid.pdf.

[158] Kluever, Craig A. Dynamic systems: modeling, simulation, and control. John Wiley \& Sons, 2015.

[159] Junior, M. T., Gerges, S. N., \& Jordan, R. (2008). Analysis of brake squeal noise using the finite element method: a parametric study. Applied Acoustics, 69(2), pp. 
$147-162$.

[160] Von Wagner, U., Hochlenert, D., Jearsiripongkul, T., \& Hagedorn, P. (2004). Active control of brake squeal via "smart pads" (No. 2004-01-2773). SAE Technical Paper.

[161] Norton, R. L. Machine design: an integrated approach. 3rd.Upper Saddle River, $\mathrm{NJ}, 22,23)$

[162] Http://www.mechanicalengineeringblog.com/5369-construction-and-working-ofdisk-brakes-in-an-automobile-disk-brakes-a-type-of-mechanical-brakingsystem/\#.WlgDNrynEdU

[163] Https://commons.wikimedia.org/wiki/File:Hydraulic_disc_brake_diagram.gif\#

[164] Garcia, E., Arevalo, J. C., Munoz, G., \& Gonzalez-de-Santos, P. (2011). On the biomimetic design of agile-robot legs. Sensors, 11(12), 11305-11334. 


\section{APPENDIX}

\section{A.1 The Torque Capacity of a MR Fluid Brake Compared to A Frictional Disk Brake}

In this appendix, we have compared the torque capacity of a magnetorheological (MR) fluid brake with a conventional frictional disk brake. The comparison considers the maximum values of the design parameters for all studied brake designs. In the development of the torque-models for both brakes, a mathematical expression for the compared torque ratio is presented. For the frictional disk brake, constant pressure and constant wear theories are considered, while static torque of the MR fluid brake is considered for comparison purpose only. Throughout the analysis the outer radius of the compared brakes is assumed to be the same to ensure similarity of size, while the inner radius is selected to achieve maximum values of braking torque for both brake designs. Reasonable values of design variables for each brake are obtained from references and adopted in this study for making comparisons between the two designs.

\section{A.1.1 Introduction}

Rotating mechanical systems are typically designed to include a brake or a rotational damping device for the purposes of controlling speed. In typical applications the maximum torque capacity of the brake is used to stop the rotating system altogether. Historically the braking device has been constructed using a frictional brake design, which has often been accompanied with adverse wear and premature failure. For frictional brakes it is known that in addition to wear and high temperature problems, large and costly actuation systems for these brakes are often required. For example, the frictional disk brake in an automotive vehicle requires a brake pedal, pneumatic or 
hydraulic assistance systems, a main brake cylinder, high pressure pipes, brake fluid, a brake caliber, and brake pads. More recently, magnetorheological (MR) fluid brakes have been introduced as an alternative brake that may simplify the system design and eliminate brake wear. MR fluid brakes are designed based upon principles that govern fluid shear-stress by altering the MR fluid viscosity, which is used to resist the motion of mechanical parts. The novel property of MR fluids is that their apparent viscosity can be significantly altered by exposing the fluid to an electrically-induced magnetic field. By controlling the strength of the magnetic field, the MR fluid viscosity is easily controlled with a fast response time and a high torque-to-volume ratio.

A very good literature review for frictional brakes has been presented by Ostermeyer [22]. In his review, the author develops a second order differential equation for the dynamic coefficient-of-friction and observes unique behavior in the coefficient of friction after a sudden jump in disk speed. Other researchers have addressed problems with frictional disk-brake squeal [159] and disk-brake control and design [160]. The reader is referred to Ch.2 for good review about MR fluid brake design

Within the body of literature known to us so far, no study has been presented in which a comparison is made between the maximum torque capacity of the frictional disk brake and the maximum torque capacity of the MR fluid brake. A study of this nature is important to an engineer who is considering the replacement of a frictional brake with a more advanced MR fluid brake. The study presented here is aimed at filling this void in the literature.

In this work, we compare the torque capacity of a MR fluid brake with a frictional disk brake. In the development of the torque-models for both brakes, a mathematical 
expression for the compared torque ratio is presented. For the frictional disk brake, constant pressure and constant wear theories are considered, while static torque of the MR fluid brake is considered for comparison purpose only. Throughout the analysis the outer radius of the compared brakes is assumed to be the same to ensure similarity of size, while the inner radius is selected to achieve maximum values of braking torque for both brake designs. Reasonable values of design variables for each brake are obtained from references and adopted in this study for making comparisons between the two designs.

\section{A.1.2 Descriptions of Frictional Disc and MR fluid Brake}

\section{A.1.2.1 Frictional Disk Brake}

A schematic for a frictional disk brake is shown at the top of Fig. A-1. The lefthand-side of this figure shows the infinitesimal force $d f$ of the frictional force resulting from applying an external compressive force $F$ which is typically very large. The compression force can be produced pneumatically, mechanically, or electrically, which results in an applied pressure between the rotating surface and the stationary lining material. The torque generated by the brake is shown by the symbol $T$. Values for the coefficient-of-friction between the lining material and the rotating disk is typically between 0.15 and 0.45 . Similarly, the maximum pressure that can be applied to the lining material before being damaged is between 1,000 and 2,000 $\mathrm{kPa}$. [161].

\section{A.1.2.2 MR Fluid Brake}

A schematic for a single disk, MR fluid brake is shown at the bottom of Fig. A-1. 

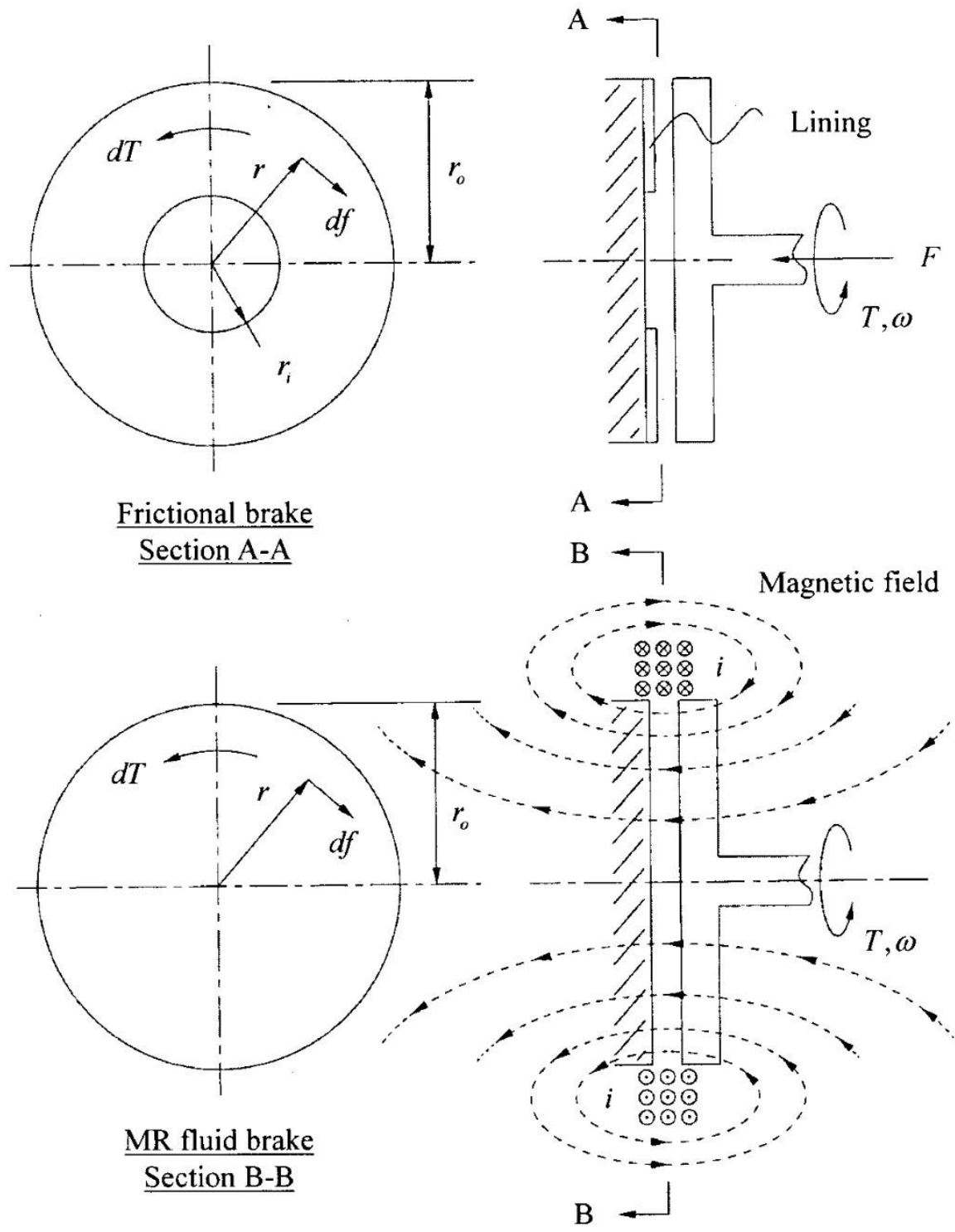

Figure A-1. A schematic showing the geometry of frictional and MR fluid brakes

The angular velocity of the disk is given by $\omega$ and the torque generated by the brake is shown by the symbol $T$. Magnetorheological (MR) fluid is shown to exist between the rotating disk and a stationary surface. The thickness of the MR fluid is not labeled in Fig. A-1 but is given by the dimension $h$ in the analysis that follows. As it turns out, in the absence of a magnetic field, MR fluid sustains a shear stress that is proportional to the 
fluid strain-rate which means that the non-energized MR fluid is Newtonian and characterized by a certain fluid viscosity. In this non-energized state the shear stress in the MR fluid generates a brake torque that is proportional to the fluid viscosity $\mu$ and the angular velocity $\omega$, while being inversely proportional to the MR fluid thickness $h$. The bottom right-hand-side of Fig. A-1 shows the MR fluid brake with a magnetic field that has been generated by passing current through the electric coil which is shown to be wrapped around the outside of the brake.

\section{A.1.3 Mathematical Modelling and Analysis}

\section{A.1.3.1 Frictional Brake Equations}

In this section the mathematical model for the frictional brake is derived and presented. Section A-A of Fig. A-1 shows a sectional view of the frictional brake disk where an infinitesimal force $d f$ is generated on the disk surface by the applied pressure. The uniform pressure and uniform wear theories are customarily used to create two mathematical models for the torque capacity of the brake [161]. For the uniform pressure theory, the pressure is assumed to be uniformly distributed over the frictional surface. For the uniform wear theory, the wear rate of the lining material is assumed to be uniform over the frictional surface. Since wear is typically proportional to contact pressure multiplied by the relative sliding velocity, the uniform wear model requires the product of contact pressure and relative sliding velocity to be constant over the frictional surface. The uniform wear model is known to yield a more conservative brake design compared to the uniform pressure model.

Uniform Pressure. Using the geometry of Fig. A-1, the infinitesimal shear force 
applied to the lining material of the frictional brake may be expressed as

$$
d f=\mu_{c} p r d r d \theta
$$

where $\mu_{c}$ is the coefficient of friction for the brake material, $r$ is the radial location of the infinitesimal shear force, $p$ is the local contact pressure between the rotating disk and the lining material, and $d \theta$ is an infinitesimal angular dimension. Using this result, it may be shown that an infinitesimal amount of torque generated by the shear force is given by

$$
d T=r d f=\mu_{c} p r^{2} d r d \theta .
$$

Eq. (A.2) may be integrated to produce the following result for the maximum torque capacity of the frictional disk brake:

$$
T_{c p}=\frac{2 \pi}{3} p_{\max } \mu_{c} r_{o}^{3} .
$$

In this result, the subscript " $c p$ " is used to denote that the brake torque has been derived based upon the constant pressure model. Also, in Eq. (A.3) $r_{o}$ is the outside radius of the lining material and the inside radius $r_{i}$ has been set to zero in order to maximize the torque capacity of the brake. In order to evaluate Eq. (A.3), reasonable values for maximum contact pressure and the coefficient of friction coefficient will be selected from Ref. [161].

Uniform Wear. As previously described, the uniform wear theory enforces a contact-pressure model that requires the product of pressure and sliding velocity to be constant over the entire frictional surface. It may be shown that this model produces a contact pressure that is inversely proportional to the radial location of the pressure point: 


$$
p=p_{\max } \frac{r_{i}}{r}
$$

where $r_{i}$ is the inside radius of the lining material. Using this result with Eq. (A.2) it may be shown that the uniform wear model produces the following result for the torque capacity of the frictional disk brake:

$$
T_{w}=\pi p_{\max } \mu_{c} r_{i}\left(r_{o}^{2}-r_{i}^{2}\right)
$$

In this result the subscript " $w$ " is used to denote that the brake torque has been derived based upon the constant wear model. In order to maximize this torque, it may be shown that the inside radius should be designed according to the following rule:

$$
r_{i}=\sqrt{\frac{1}{3}} r_{o}
$$

Substituting Eq. (A.6) into Eq. (A.5) produces the following result for the maximum torque capacity of the frictional disk brake using the uniform wear model:

$$
T_{w}=\frac{2 \pi}{3 \sqrt{3}} p_{\max } \mu_{c} r_{o}^{3}
$$

\section{A.1.3.2 MR Fluid Brake Equations}

In this section the mechanical and electrical models for the MR fluid brake are derived and presented. Section B-B in Fig. 1 shows a sectional view of the single MR fluid brake disk. The steps for developing the mathematical modeling of the current MR fluid brake design is similar to the MR fluid brake presented in Ch.6. It is important to note that the inner radius of a frictional surface of the rotating disc has been set to zero in order to maximize the torque capacity of the brake. Equation (6.15) can be given as 


$$
T_{M R}=\frac{\pi}{2} \frac{\mu \omega}{h} r_{o}^{4}+\frac{2 \pi}{3} \tau_{B} r_{o}^{3}
$$

where the applied current is at its maximum value. The first term on the right-hand-side of Eq. (A.8) represents the resistance applied by the non-energized brake while the second term represents the additional resistance that is applied by exposing the MR fluid to the maximum strength of the magnetic field. Typically, the fluid film thickness $h$ is large so that the non-energized brake torque is small. In the comparisons that follow, the static braking torque will be considered which neglects the first term in Eq. (A.8) entirely.

\section{A.1.4 Brake Comparisons}

As mentioned earlier, the main objective of this Technical Brief is to compare the maximum torque capacity for the MR fluid brake to that of the frictional disk brake for designs of similar size. In order to make this comparison, we will assume that each brake holds its load in a stationary condition (i.e., $\omega=0$ ) and we will evaluate the ratio of the MR fluid brake torque capacity to the frictional brake torque capacity. These results are given by

$$
\frac{T_{c p}}{T_{M R}}=\frac{p_{\max }}{\tau_{B}} \mu_{c} \quad \text { and } \quad \frac{T_{w}}{T_{M R}}=\sqrt{\frac{1}{3}} \frac{p_{\max }}{\tau_{B}} \mu_{c}
$$

The first thing we notice about the relative brake torque between the frictional brake and the MR fluid brake is that the ratio is independent of geometry. In other words, the relative braking torque is independent of the outer brake radius $r_{o}$ which means that the comparisons in Eq. (A.9) are valid for similar brakes of any size. Physically, there are only three dimensional parameters that that must be used to make this comparison: 1) the 
maximum contact pressure that may be sustained by the frictional brake lining material $\left.p_{\max }, 2\right)$ the coefficient of friction for the lining material $\mu_{c}$, and 3) the maximum yield shear-stress for the MR brake fluid $\tau_{B}$.

From machine design textbooks [161] we see that molded or sintered metal linings can withstand a maximum contact pressure given by $p_{\max }=2,000 \mathrm{kPa}$ while operating with a coefficient of friction given by $\mu_{c}=0.45$. Similarly, the maximum shear yield-stress for MR fluids is given by $\tau_{B}=50 \mathrm{kPa}$ [157]. Substituting these characteristic values into Eq. (A.9) produces the following comparison between the frictional brake and the MR fluid brake:

$$
\begin{aligned}
& \frac{T_{c p}}{T_{M R}}=\frac{p_{\max }}{\tau_{B}} \mu_{c}=\frac{2,000 \mathrm{kPa}}{50 \mathrm{kPa}} \times 0.45=18.00, \text { and } \\
& \frac{T_{w}}{T_{M R}}=\sqrt{\frac{1}{3}} \frac{p_{\max }}{\tau_{B}} \mu_{c}=\sqrt{\frac{1}{3}} \frac{2,000 \mathrm{kPa}}{50 \mathrm{kPa}} \times 0.45=10.39 .
\end{aligned}
$$

From these calculations it is shown that the torque capacity of the frictional brake is 10 to 18 time greater than the torque capacity for the MR fluid brake of similar size. Another way to consider this result is to recognize that in order to achieve the same torque output, the outer radius of the MR fluid brake would need to be 2.18 times larger than the outer radius of the frictional brake as determined from the uniform pressure model. Similarly, the outer radius of the MR fluid brake would need to be 2.62 times larger than the outer radius of the frictional brake as determined from the uniform wear model. In general terms we would say that the MR fluid brake must be twice the size of the frictional disk brake in order to achieve the same braking torque. The numerical values of some design parameters used for this analysis are given in the next section. 


\section{A.2 Numerical Values for Some Design Parameters}

The following design parameters are presented as typical characteristics for a MR fluid brake. These values are physically realistic and may be used to satisfy the design constraint shown in Eq. (6.19). Note: design parameters for the electric coil have not been included here.

\begin{tabular}{|l|c|c|c|}
\hline \multicolumn{1}{|c|}{ Description } & Symbol & Value & Units \\
\hline Nominal MR fluid thickness & $h_{o}$ & 50 & $\mu \mathrm{m}$ \\
\hline Mass moment-of-inertia & $J$ & 0.064 & $\mathrm{~kg} \mathrm{~m}^{2}$ \\
\hline Disk radius & $R$ & 95 & $\mathrm{~mm}$ \\
\hline Shaft radius & $R_{s}$ & 20 & $\mathrm{~mm}$ \\
\hline Nominal brake torque & $T_{o}$ & 53 & $\mathrm{~N} \mathrm{~m}$ \\
\hline Characteristic time constant & $t_{o}$ & 0.25 & $\mathrm{~s}$ \\
\hline MR fluid viscosity & $\mu$ & 0.1 & $\mathrm{~Pa} \mathrm{~s}$ \\
\hline $\begin{array}{l}\text { MR fluid maximum shear } \\
\text { stress }\end{array}$ & $\tau_{B}$ & 30 & $\mathrm{kPa}$ \\
\hline Nominal shaft speed & $\omega_{o}$ & 2,000 & $\mathrm{rpm}$ \\
\hline
\end{tabular}




\section{VITA}

Salwan Obaid Waheed Khafaji born in May 1979 at Babil-IRAQ. He finished his undergraduate and master studies at University of Babylon-IRAQ in 2003 and 2005, respectively. In 2011, Salwan got a scholarship offered by HCED- IRAQ followed by the prime minister of IRAQ to pursue his Ph.D. study in USA. In January 2014, he started his study at English Intensive Program (IEP) offered by the University of Missouri, Columbia (MU-USA). After he finished IEP in one semester, he transferred to academic study in August 2014 in the Mechanical and Aerospace Engineering at MU. In his Ph.D. research, Salwan worked on sensitivity analysis of a magnetorheological fluid brake and he got two optimal designs and disclose a file for patent. He published several papers and he got two papers under review. He gained recognition from his supervisor because of that. Furthermore, Salwan is chosen to be a reviewer for several scientific journals, for example, Journal of Mechanical Science and Technology (JMST).

In addition, Salwan is proficient with mechanical design and finite-element methods for dealing with engineering problems. He worked on smart material modeling (MRF modeling) and getting an optimal design for both MRF brake and dampers. Salwan got his Ph.D. with honor May 2018. 